\author{
ZENTRUM \\ FÜR BIODIVERSITÄT UND NACHHALTIGE LANDNUTZUNG \\ SEKTION \\ Biodiversität, ÖKOlogIE UND NATURSCHUTZ \\ - Centre of Biodiversity and Sustainable Land Use - \\ SECTION: BIOdIVERsity, ECOLOgy and NATURE Conservation
}

\title{
Plant species and global change agents as driving factors of rhizosphere processes and soil nematode communities
}

\author{
Dissertation zur Erlangung des Doktorgrades der \\ Mathematisch-Naturwissenschaftlichen Fakultäten der \\ Georg-August-Universität Göttingen
}

\author{
vorgelegt von \\ Diplom-Biologin \\ Simone Cesarz
}

aus

Göttingen 
Referentin/Referent:

Korreferentin/Korreferent: Prof. Dr. Matthias Schaefer

Tag der mündlichen Prüfung: $\quad$ 13.4.2012 
Wer Würmer hat, ist nie allein 


\section{Table of Content}

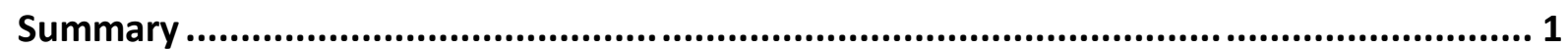

\section{Chapter 1}

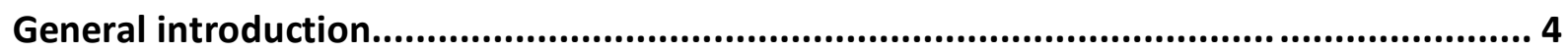

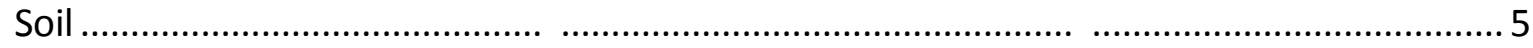

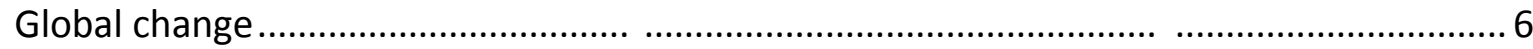

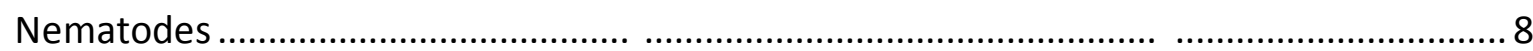

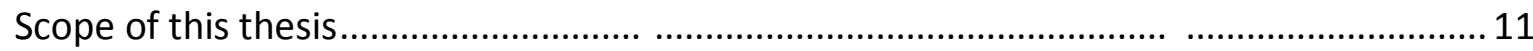

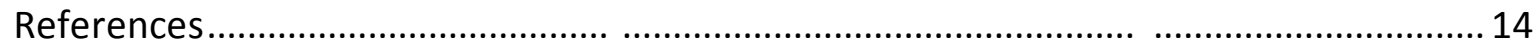

\section{| Chapter 2}

Tree species diversity versus tree species identity: Driving forces in structuring forest food webs as indicated by soil nematodes........................................................... 19

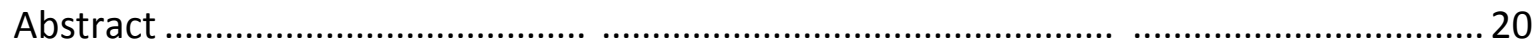

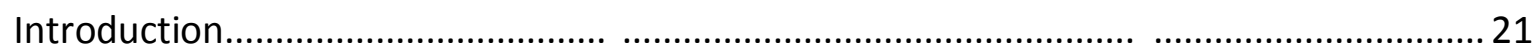

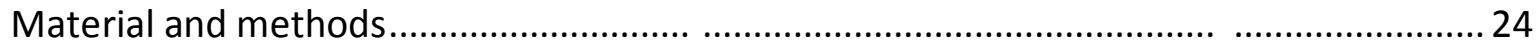

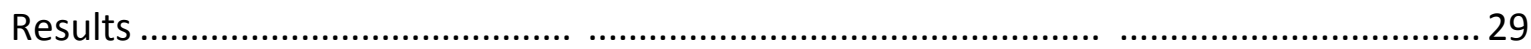

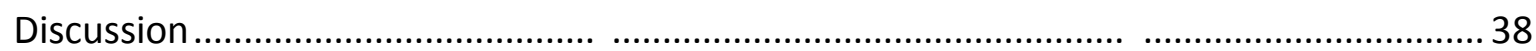

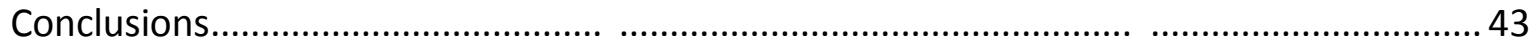

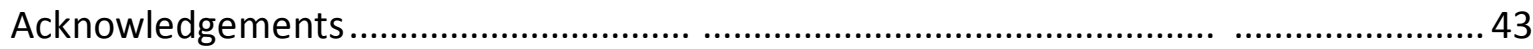

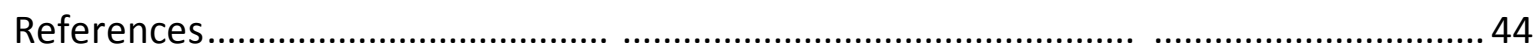

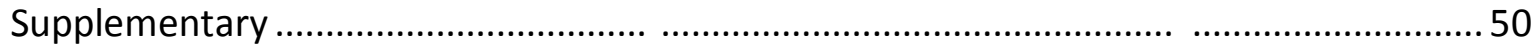




\section{| Chapter 3}

Roots from beech (Fagus sylvatica L.) and ash (Fraxinus excelsior L.)

differentially affect soil microorganisms and carbon dynamics ....................................... 53

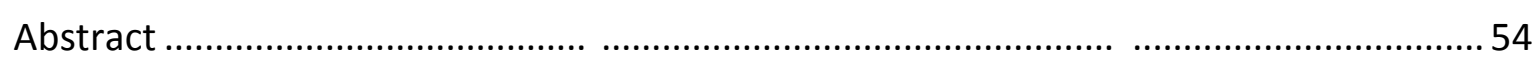

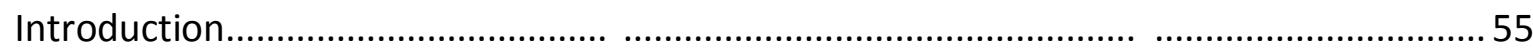

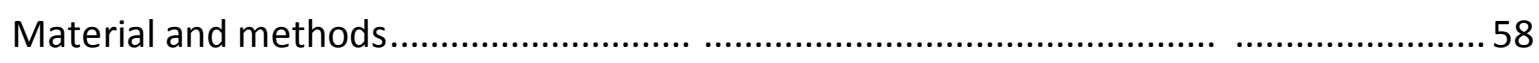

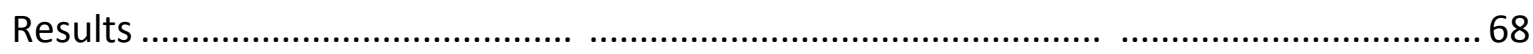

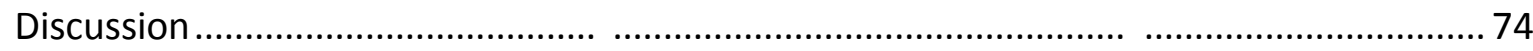

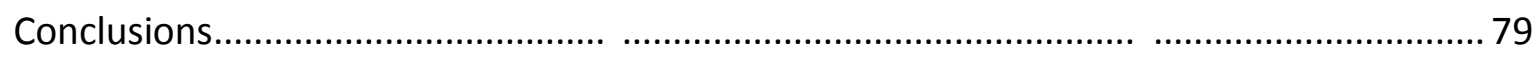

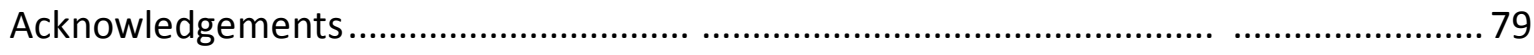

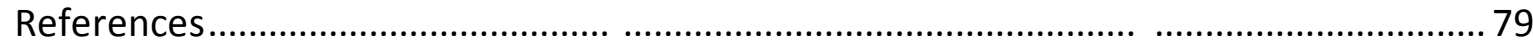

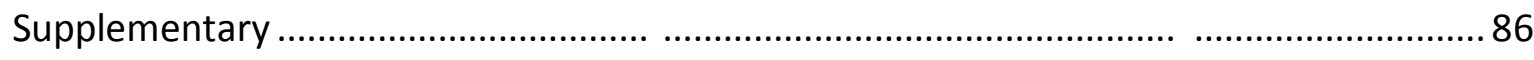

\section{Chapter 4}

Global change belowground: impacts of elevated $\mathrm{CO}_{2}$, nitrogen, and summer drought on soil food webs and biodiversity ................................................................. 90

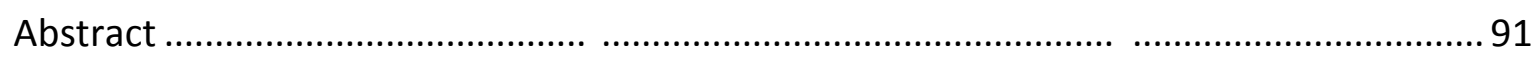

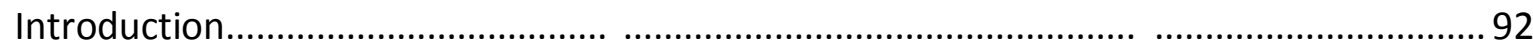

Materials and methods ........................................................................................... 94

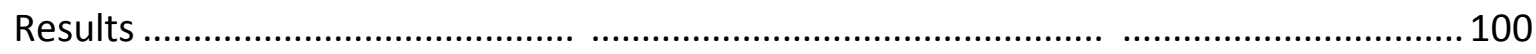

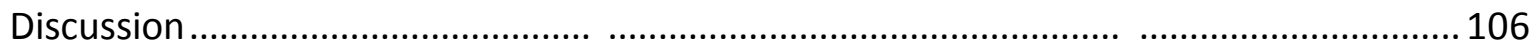

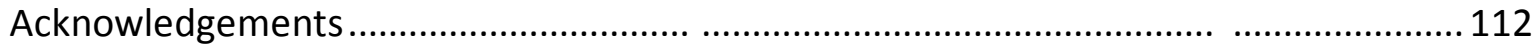

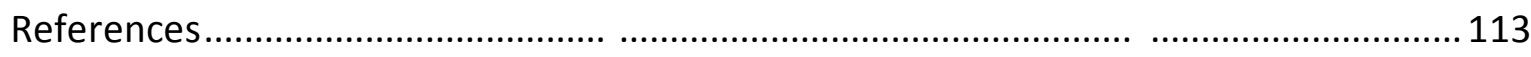

\section{Chapter 5}

Changes in soil processes indicated by soil nematodes: Interactive responses to elevated $\mathrm{CO}_{2}$, nitrogen deposition and reduced precipitation .....................................120

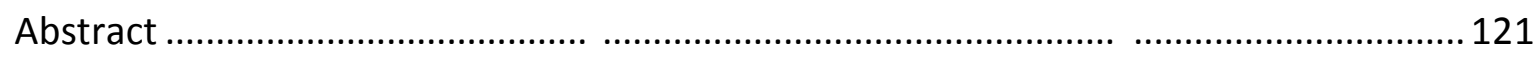

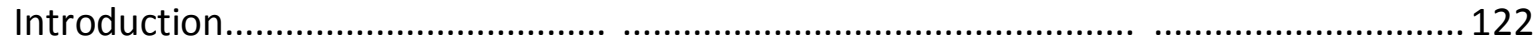

Material and methods............................................................................................ 126

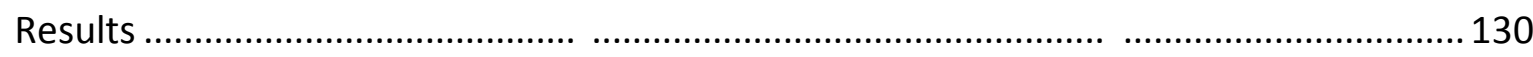




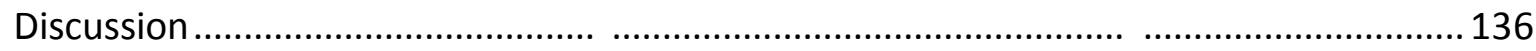

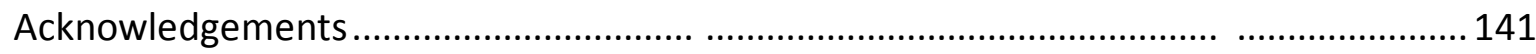

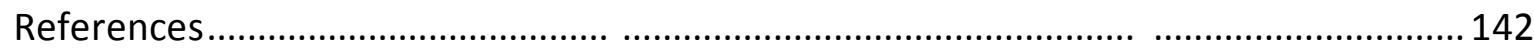

\section{| Chapter 6}

First step to integrate nematodes into food web analysis using NanoSIMS

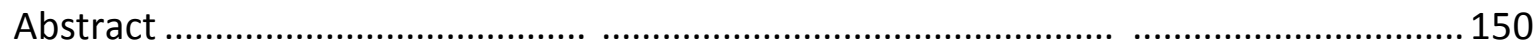

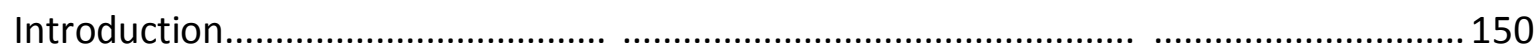

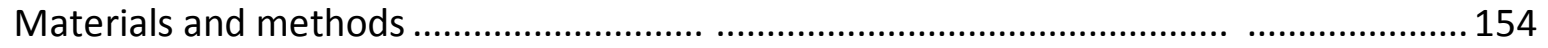

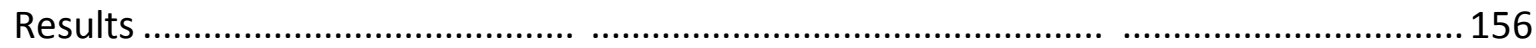

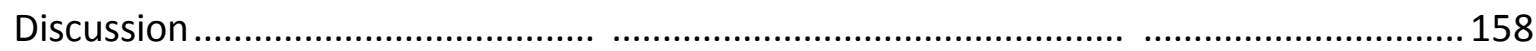

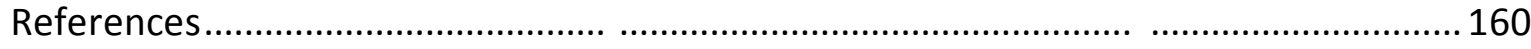

\section{Chapter 7}

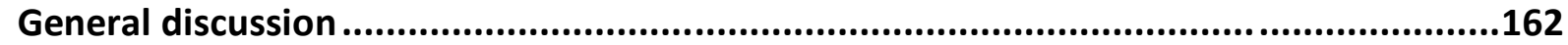

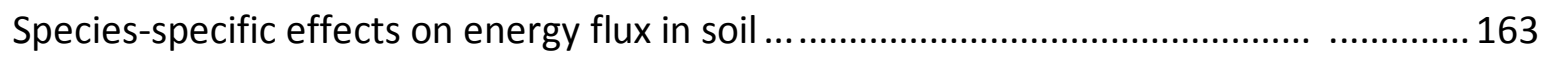

Global change and belowground communities ...................................................... 165

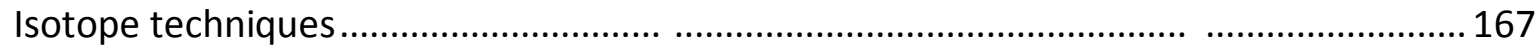

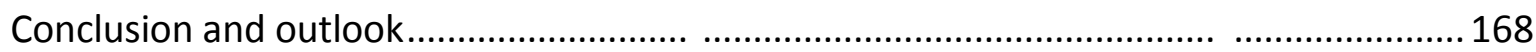

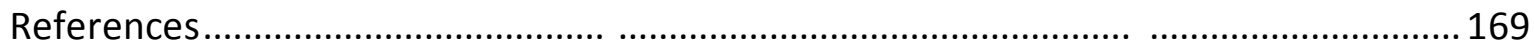

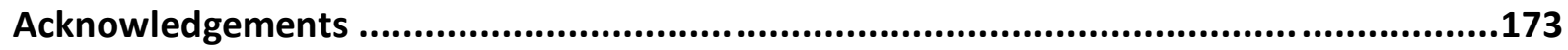

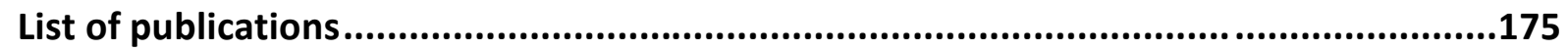

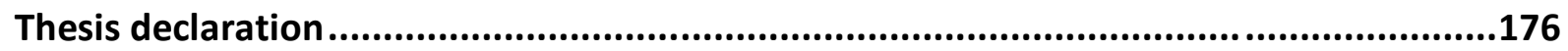

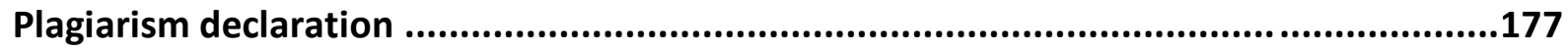




\section{Summary}

The soil system harbors important ecosystem functions. Understanding the factors influencing the functioning of soil systems is crucial to react adequately on changes induced by alteration in plant species compositions and climate change. Soil food webs deliver important information on energy flux in soil and can help to understand global carbon and nitrogen fluxes. To date, the effect of tree species identity and diversity as well as the effect of speciesspecific root- and litter-mediated effects have not been investigated on the compartmentalization of basal resources, i.e., bacteria and fungi, and on the way carbon and nitrogen is channeled through the soil system. There is also lack of information on how human induced climate change influences processes in soil. Although soil stores at least twice as much carbon as the atmosphere and plants together, the response of the belowground system to interactively acting global change agents has not been investigated so far. Carbon stocks in soil are likely to be influenced by the response of the belowground system to global change agents thereby potentially reinforcing atmospheric $\mathrm{CO}_{2}$ concentrations by accelerating the loss of soil carbon.

In Chapter 2, the effect of tree identity and diversity was investigated on the structure of soil nematodes. The results suggest that tree species identity is more important than tree species diversity in structuring nematode communities and associated soil processes. Ash increased the density of bacterial-feeding nematodes and reduced the number of fungal feeders, indicating distinct changes in regulatory forces of soil food webs. Beech detrimentally affected bacterial feeders but favored fungal feeders probably via $\mathrm{pH}$-mediated increase in the fungalto-bacterial ratio. The effect of lime was less pronounced but tended to be generally negative. The results indicate that both leaf litter and roots influence the nematode community, with the effects being driven not solely by resource quality but additionally by availability, i.e., seasonal shifts in dominant belowground resources. In sum the structure of soil food webs varies markedly with tree species pointing to the importance of variations in plant resources, i.e., leaf litter quality and root exudates, as strong bottom-up regulating factors of microbial communities and energy channels of decomposer systems.

In Chapter 3, we used ${ }^{13} \mathrm{C}$ and ${ }^{15} \mathrm{~N}$ labeled ash litter to trace the flux of carbon and nitrogen through soil influenced by beech and ash seedlings. We found that beech decreased soil $\mathrm{pH}$ by root exudation thereby increasing fungal biomass and decreasing carbon use efficiency of 
bacteria. Higher bacterial respiration induced carbon loss from soil and prevented litterderived carbon and nitrogen to reach higher trophic levels. In contrast, ash had no effect on microbial or soil parameters although its root biom ass exceeded that of beech markedly. Ash was, however, very efficient in using litter-derived nitrogen from soil. The results suggest that beech and ash differentially impact soil processes with beech affecting the belowground system predominantly via roots whereas ash predominantly via litter.

In Chapter 4, the effects of increased atmospheric $\mathrm{CO}_{2}$, elevated nitrogen and reduced precipitation were investigated on the composition and diversity of belowground animal communities. Elevated $\mathrm{CO}_{2}$ increased microbial biomass by increased rhizodeposition leading to increased densities of ciliates, collembolans and gamasid mites. Reduced precipitation was of minor importance as the soil community in the sandy soils of the study site is probably adapted to drought conditions. Nitrogen addition ne gatively affected predatory and herbivorous nematodes, and overall nematode diversity. $\mathrm{CO}_{2}$ and nitrogen interactively affected nematode communities resulting in taxonomically and functionally altered, potentially simplified, soil communities.

In Chapter 5, we used functional guilds of nematodes to inspect changes in soil processes induced by increased atmospheric $\mathrm{CO}_{2}$ concentrations, elevated nitrogen levels and reduced precipitation. We found that the decomposer community switched from a bacterial dominated to a more fungal dominated system at elevated $\mathrm{N}$ indicating strong changes in the microbial community and the functioning of belowground food webs. Nitrogen fertilization also reduced top-down forces and simplified soil food webs exposed to additional $\mathrm{N}$ input. Further, bacterial-feeding nematodes were not able to profit from increased microbial biomass suggesting that quality rather than quantity of food resources controls nematode densities. Reduced densities of root-feeding Longidoridae likely reflected increased belowground plant defense at high $\mathrm{CO}_{2}$ and $\mathrm{N}$ levels. Changes in decomposition processes shifting towards fungal domination at elevated $\mathrm{N}$ levels indicating an increase in recalcitrant and resistant compounds contributing to $C$ sequestration thereby attenuating the increase in atmospheric $\mathrm{CO}_{2}$ concentrations.

In Chapter 6, NanoSIMS is introduced as a technique to measure the isotopic signature of single nematodes. I developed embedding techniques of nematodes for measurements in 
high vacuum and present first results but also restrictions. Further, different fields of application for tracing elements in food webs are presented. 


\section{| Chapter 1}

\section{General Introduction}

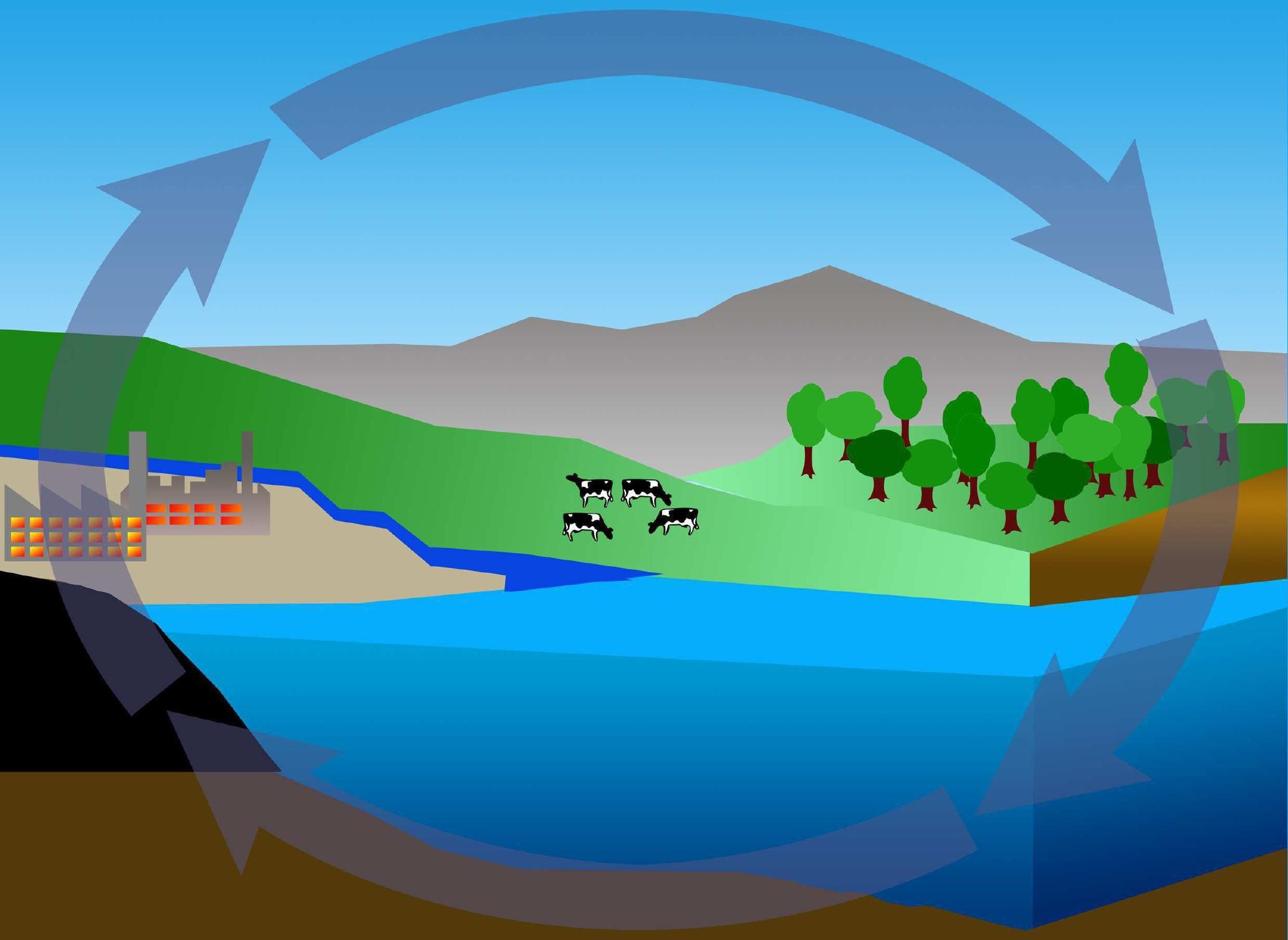




\section{Soil}

The soil is an important component within the global carbon cycle harboring main ecosystem functions such as decomposition and mineralization of nutrients, it also sequesters high amounts of carbon. The acquisition of data on soil carbon stocks, as well as fluxes into and out of the soil organic matter pool, are relatively easy to obtain. However, the way carbon is processed within the soil system is not well understood due to its complexity. This is unfortunate as the fate of carbon in soil and the factors driving its dynamics are crucial for the understanding of ecosystem functioning and to realistically predict global change processes.

The complexity of soil can be ascribed to different pore sizes, rich-textured surfaces and diverse organic and inorganic compounds leading to a heterogeneous environment. In addition, an enormous diversity of soil organisms coexists in soil with a high degree of possible interactions among each other and with plants. Plan ts deliver organic material to soil, forming the basis of the decomposer system which is responsible for important ecosystem functions.

The plant-derived energy input for soil organisms consists predominantly of litter and rhizodeposits. Almost $90 \%$ of the aboveground biomass produced annually enters the soil food web (Gessner et al., 2010). Litter characteristics are mainly responsible for soil properties and microbial activity and are relatively well investigated (Reich et al., 2005; Langenbruch et al., 2012). High quality litter is characterized by low C-to-N ratios, low lignin and high calcium and magnesium content, and high decomposition rates. Low quality litter comprises high Cto-nutrient ratios due to high amounts of recalcitrant compounds such as lignin (Hättenschwiler et al., 2005a; Jacob et al., 2009, 2010) and, therefore, mitigates decomposition and mineralization processes (Swift et al., 1979).

Recent studies highlighted that root-derived nutrie nt input is of major importance (Pollierer et al., 2007; Paterson et al., 2011). In studies with hydroponic cultures, 98\% of the root exudates originated from root tips (Farrar et al., 2003) and were shown to comprise compounds of different complexity. The majority of root exudates comprise labile compounds such as sugars, organic acids and amino acids (Paterson et al., 2009). Root exudates account for 1-10\% of photoassimilates of plants (Grayston et al., 1996; Farrar et al., 2003) and differ in quality and quantity (Jones et al. 2004). Field data on species-specific exudation patterns are hampered by methodological problems and therefore up to now only data from laboratory 
systems are available. Rhizodeposition was shown to improve nutrient acquisition (Koranda et al., 2011; Paterson et al., 2011), influence the chemical and physical structure of soils (Gregory, 2006) and structure microbial communities (Söderberg et al., 2004; Paterson et al., 2007).

Both litter and root-derived energy has been suggested to be compartmentalized into a bacterial, fungal and root (mycorrhiza and roots) energy channel (Moore and Hunt, 1988; Pollierer et al., 2009). Bacteria were shown to predominantly use the labile fraction of litter and roots (Paterson et al., 2008). The bacterial chain was proposed to be relatively short due to high turnover rates and to predominate in grasslands (Scheu and Setälä, 2002). The importance of fungi increases in forests and the contribution of mycorrhiza to soil processes is suggested to be of high importance for soil food webs (Pollierer et al., 2007, 2009). In temperate and boreal systems woody species are colonized predominantly by ectomycorrhizal (EM) fungi, while grasses and herbaceous plant species are colonized by arbuscular mycorrhizal (AM) fungi. Both mycorrhiza types differ in nutrient acquisition. EM fungi are reported to predominantly acquire nitrogen and AM fungi phosphorous with both trading nutrients for carbon with plants (Smith and Read, 2008). Mycorrhiza are estimated to account for $80 \%$ of the fungal biomass and $30 \%$ of the whole microbial biomass in boreal forests (Wallander et al. 2001; Högberg \& Högberg 2002) and may play an important role in fueling soil food webs in forests (Pollierer et al., 2007). However, evidence for direct feeding relationships between mycorrhiza and soil fauna is still scarce as ectomycorrhiza cannot be separated easily from saprotrophic fungi.

A major aim of today's soil ecological research is to identify the relationships between organisms in soil and the factors by which they are influenced aiming to better understand ecosystem functioning. Increasing knowledge on soil functioning is crucial in a changing world and may help to react adequately on altered soil processes.

\section{Global change}

The understanding of species-specific effects and their related energy input into soil (litter vs. roots) is of high importance to understand terrestrial ecosystems, especially in a global change context, as soils can function as carbon sinks or carbon sources (Schulze and Freibauer, 2005). 
Alterations in atmospheric $\mathrm{CO}_{2}$ concentration, nitrogen deposition and precipitation regime are important global change drivers and understanding their interactive effects are crucial to realistically predict global change processes.

The concentration of $\mathrm{CO}_{2}$ in the atmosphere rises continuously as a result of enhanced combustion of fossil fuels, cement production and I and use change (McKinley et al., 2011). Since the industrial revolution, atmospheric $\mathrm{CO}_{2}$ concentrations have increased from 270 ppm to 379 ppm in 2005 and will reach 550 ppm by the year 2050 (Indermühle et al., 1999; IPCC, 2007). $\mathrm{CO}_{2}$ is the raw material of photosynthesis and is known to affect plant growth and development due to increased carboxylation rates. Overall aboveground biomass increases and was shown to be strongest in trees, followed by legumes and is lowest in grasses but did not change in $\mathrm{C}_{4}$ plants (Ainsworth and Long, 2005). Elevated $\mathrm{CO}_{2}$ also reduces stomatal conductance and increases water use efficiency and, therefore, decreases water uptake in plants (Ainsworth and Long, 2005).

It is likely that the above described plant responses propagate to the belowground system. Unfortunately, the response of the belowground system to inhanced $\mathrm{CO}_{2}$ concentrations in the atmosphere are only little understood. Positive effects of elevated $\mathrm{CO}_{2}$ on soil microbial biomass was reported (Hu et al., 2001; Carney et al., 2007; He et al., 2010) and this is in particular induced by increased rhizodeposition (Zak et al. 1993; Adair et al. 2009). Faunal reactions to elevated $\mathrm{CO}_{2}$ are complex and depend on ecosystem and plant community (Blankinship et al., 2011), but are generally little investigated. The quality of plant residues entering the soil often decreased at elevated $\mathrm{CO}_{2}$ concentration as the $\mathrm{C}$-to- $\mathrm{N}$ ratio increases at low nitrogen levels (Körner, 2000).

However, human activity increases terrestrial $\mathrm{N}$ levels significantly and may induce unexpected $\mathrm{CO}_{2}$ responses (Reich, Hobbie, et al., 2006a). Nitrogen additions were reported to negatively affect biodiversity (Clark and Tilman, 2008; Reich, 2009), decrease the amount of rhizodeposition (Dijkstra et al., 2005; Högberg et al., 2010), but to increase aboveground plant biomass, while elevated $\mathrm{CO}_{2}$ enhances belowground biomass (Reich, Knops, et al., 2001). Changes in quality and composition of plant resourc es to the soil are likely to propagate into soil fauna and induce changes in soil processes. Th is may have major consequences on carbon stocks and may reinforce climate change by increasing the biological activity in soil due increased plant resource pools and by priming (Kuzyakov, 2010). Otherwise, imposed top- 
down effects may counteract increased bottom-up effects resulting in negative feedbacks and thus in carbon sequestration (Wardle et al., 1998).

\section{Nematodes}

Nematodes are ubiquitous, abundant and diverse. The y are common in terrestrial and aquatic habitats from the arctic to deserts (Bongers and Ferris, 1999). Typically, nematodes are about $0.3 \mathrm{~mm}$ to $3 \mathrm{~mm}$ long with some exceptions in animal parasites. Estimates on species numbers vary between 40,000 and almost astronomic 10,000,000 (Blaxter, 1998). In terrestrial systems the density of nematodes typically ranges between $10^{5}$ and $10^{6}$ ind. $\mathrm{m}^{-2}$ (Yeates, 1999). Free living nematodes (others are animal parasites and plant-parasitic forms) evolved diverse feeding modes comprising bacterial, fungal and plant feeders, predators, omnivores and entomophagous species (Yeates et al. 1993). Diverse feeding habits and easy cultivation and sampling made them ideal bioindicators as free living soil nematodes represent habitat conditions and sensitively respond to environmental changes. Consequently, a range of indices were developed to make use of nematodes as bioindicators. Bongers (1990) proposed the Maturity Index (MI) and arranged nematodes into 5 classes according to their life stage strategy in colonizers $(c)$ and persisters $(p)$ :

The class of $c-p 1$ nematodes comprises small and abundant species with short generation times. They produce a large number of offspring and this is reflected by a large proportion of the body occupied by gonads. They are able to form Dauer larvae when nutrient pools decrease and are tolerant to pollutants. All nemato des in $c-p 1$ are bacterial feeders.

Nematodes of $c-p 2$ have a longer generation time and slightly lower reproductive rates. They are often described as basal nematodes (Ferris et al. 2001) as they are generally very abundant and ubiquitous. They do not form Dauer larvae but a re relatively tolerant to disturbance and pollutants. Bacterial and fungal feeder occur in th is group. 


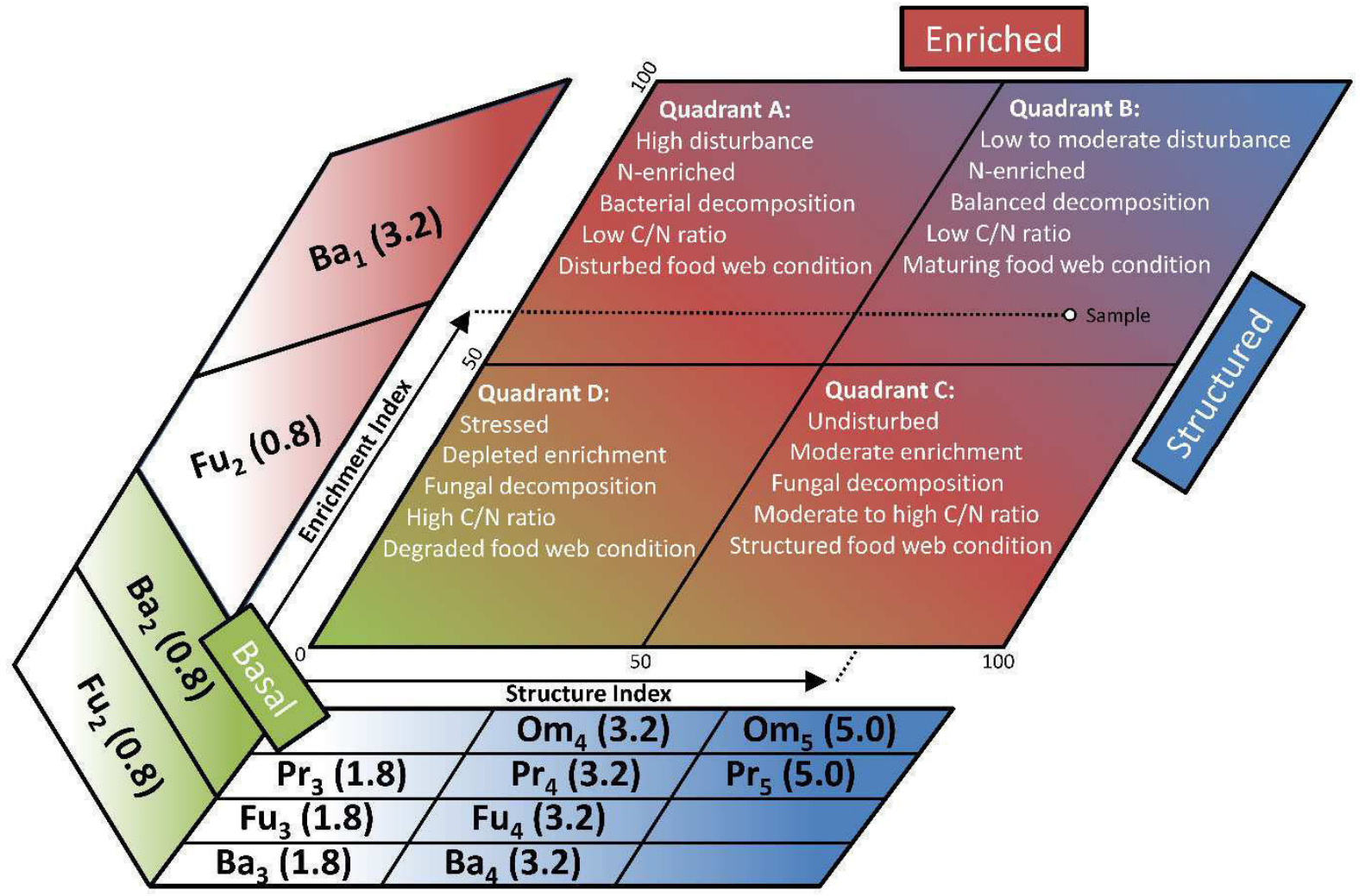

Fig 1 Graphic representation of the nematode faunal profile. Structure and enrichment within soil food webs are calculated using functional nematode guilds and a distinct weighting (numbers in brackets) according their life history characteristics along an Enrichment Index (EI) and Structure Index (SI). Calculated values represent condition within food webs and are described in dif ferent quadrants along the enrichment and structure index. Modified after Ferris et al. ( 2001).

Nematodes of $c-p 3$ have longer generation times than nematodes of $c-p 2$, lower fecundity and react more sensitively to disturbance. Predatory nematodes complement bacterial and fungal feeders in this group.

Nematodes in class $c-p 4$ are bigger and have a relatively long generation time. They react sensitively to disturbance and have a permeable cuticle. Larger fungal feeders, bacterial feeders and predatory nematodes are included in this group, as well as small omnivores.

Only large dorylaimid species are ascribed to $c-p 5$ nematodes. They have a long life span, produce few offspring and large eggs. Only a low proportion of their body mass is occupied by gonads. Their cuticle is permeable and they react very sensitively to disturbance and pollutants. Only predators and omnivores are present in this group.

After assigning nematodes in the respective $c-p$ classes, the $\mathrm{MI}$ can be calculated as the weighted proportion of nematodes: 
$M I=\Sigma v(i) \times f(i)$,

with $v(i)$ being the $c-p$ value of the taxon and $f(i)$ the frequency of the taxon within the sample. High MI values indicate stable environments and low MI disturbed and nutrient enriched sites.

Plant feeders are not considered within the MI, but Bongers (1990) proposed the Plant Parasitic Index (PPI) which reflects the enrichment of the soil thereby benefiting plants ; PPI often is inverse to the MI. Functional guilds were proposed by Ferris et al. (2001) allowing to compare ecosystem processes and refine the $\mathrm{MI}$ from Bongers (1990). A functional guild is characterized by the respective feeding type of bacterial feeders (Ba), fungal feeders (Fu), predators $(\mathrm{Pr})$ and omnivores $(\mathrm{Om})$ and the respective $c-p$ value, e.g., $\mathrm{Ba}_{2}$ and $\mathrm{Om}_{4}$ are bacterial feeding nematodes in $c-p 2$ and omnivores in $c-p 4$, respectively. Different nematode guilds are suggested to represent different soil conditions, i.e., basal conditions, enrichment and structure. Basal conditions (b) are represented by $\mathrm{Ba}_{2}$ and $\mathrm{Fu}_{2}$ as they have a wide ecological range and are therefore, ubiquitous. Enrichment (e) is expressed by $\mathrm{Ba}_{1}$ and $\mathrm{Fu}_{2}$ nematodes and provides information on the resource status of the ecosystem investigated. $\mathrm{Ba}_{1}$ nematodes occur in disturbed systems in high numbers, e.g., due to mortality of other organisms leading to nutrient flushes and high micr obial activity. High availability of complex organic material is reflected in increased densities of Fu 2 . Therefore, both guilds represent nutrient enriched conditions. Structure (s) is expressed by nematodes of higher $c-p$ values ranging from 3 to 5 . Nematodes within these guilds indicate stable conditions, e.g., recovering from stress and higher food web connectivity and therefore, point to structured ecosystems with many trophic links. These indications are form alized in the Enrichment Index (EI) and the Structure Index (EI) and provide a nematode faunal profile (Fig. 1). For calculation, densities of functional guilds are weighted (w) according to their basal (b), enrichment (e) and structural (s) components:

$\mathrm{b}=\left(\mathrm{Ba}_{2}+\mathrm{Fu}_{2}\right) \times \mathrm{wn}_{\mathrm{n}}$

$\mathrm{e}=\left(\mathrm{Ba}_{1} \times \mathrm{w}_{\mathrm{n}}\right)+\left(\mathrm{Fu}_{2} \times \mathrm{w}_{\mathrm{n}}\right)$

$s=\left(B a_{n} \times w_{n}\right)+\left(F u_{n} \times w_{n}\right)+\left(P r_{n} \times w_{n}\right)+\left(O m_{n} \times w_{n}\right)$,

with $\mathrm{n}$ representing the corresponding $c-p$ values with $\mathrm{w}_{\mathrm{c}-\mathrm{p} 1}=3.2, \mathrm{w}_{\mathrm{c}-\mathrm{p} 2}=0.8, \mathrm{w}_{\mathrm{c}-\mathrm{p} 3}=1.8$, $w_{c-p 4}=3.2$ and $w_{c-p 5}=5.0$. 
The weighting is based on taxonomic richness in foo d webs of different complexity. It relies on the hypothesis of constant connectance in community food webs giving that the number of trophic links increases constantly with the square of the number of species (Cohen, 1989; Martinez, 1992) and is then related to the number of $c-p$ classes. The rationale is explained in detail in Ferris et al. (2001).

Calculated $\mathrm{EI}$ and $\mathrm{SI}$ result in a faunal profile which indicates different conditions within the soil food web (Fig. 1). El and SI are calculated as:

$E I=100 \times(e /(e+b))$

$S I=100 \times(s /(s+b))$

Additionally, the Channel Index $(\mathrm{Cl})$ is often calculated and reflects the percentage of fungal feeding nematodes among the total of fungal feeders and opportunistic bacterial feeding nematodes providing information on the dominant decomposition channel.

It is calculated as:

$\mathrm{Cl}=100 \times\left(0.8 \times \mathrm{Fu}_{2} /\left(3.2 \times \mathrm{Ba}_{1}+0.8 \times \mathrm{Fu}_{2}\right)\right.$,

and can help to identify changes in decomposition processes. High $\mathrm{Cl}$ values indicate dominance of fungal decomposition, while low values indicate bacterial dominated decomposition.

\section{Scope of this thesis}

The objectives of this thesis were to investigate the effect of different tree species on energy flow in forest soils and the effect of litter and root-derived resources on rhizosphere processes (Chapter 2 and 3). The effect of global change agents $\left(\mathrm{CO}_{2}\right.$, nitrogen, precipitation) on soil processes was investigated in grassland (Chapter 4 and 5). In both chapters, nematodes were used as indicators representing the response of the soil food web. In addition, the isotopic signature of nematodes was measured for the first time using NanoSIMS (Chapter 6) to establish a novel tool for integrating nematodes in to food web analysis.

In the following the main hypotheses are outlined and an overview about every chapter is given. 


\section{Main hypotheses}

(1) Tree diversity positively affects the abundance of soil nematodes due to an increase in resource partitioning, whereas tree species identity affects specific nematode groups due to differences in litter and root quality (Chapter 2).

(2) Carbon and nitrogen flow in soil food webs is influ enced by tree species identity with the induced changes altering microbial community composition and these changes propagate to higher trophic levels (Chapter 3).

(3) Global change agents interactively influence the structure of soil animal communities (Chapter 4).

(4) Global change agents differently affect nematode functional guilds due to differently affecting soil processes (Chapter 5).

In Chapter 2, the effect of the presence of beech (Fagus sylvatica), ash (Fraxinus excelsior) and lime (Tilia cordata) via their litter and fine roots were investigated on the bacterial and fungal energy channel using nematodes as indicators. Clusters of three trees were established varying in tree species numbers and tree species composition to separate identity and diversity effects of the studied tree species. Tree identity specifically influenced nematode trophic groups and functional guilds, while tree diversity led to balanced bacterial and fungal energy channels indicating food web stability. Pure beech clusters beneficially affected fungal feeding Leptonchidae $\left(\mathrm{Fu}_{4}\right)$ by delivering recalcitrant beech litter, therefore promoting fungi. The contribution of other tree species, especially ash, detrimentally affected fungal feeders. Ash, however, increased bacterial feeders significantly being ascribed to its fine root influence. The effect of lime was generally negative except for plant feeders. A switch in the importance of litter and root-derived resources were suggested due to seasonal changes in nutrient availability. The results indicate that the structu re of soil food webs varies markedly with tree species identity and point to the importance of basal resources i.e., litter quality and root resources, suggesting strong bottom-up control of how energy is channeled through decomposer systems.

In Chapter 3, the effect of beech and ash trees on the flow of carbon and nitrogen into the soil was investigated in a laboratory study. ${ }^{13} \mathrm{C}$ and ${ }^{15} \mathrm{~N}$ labeled ash litter was added to rhizotrons planted without trees, with two beech trees, two ash trees and a mixture of both tree species 
allowing to separate root derived and species specific effects. Compound-specific ${ }^{13} \mathrm{C}$-PLFA analysis indicated a stronger contribution of fungal biomass in beech rhizotrons being also stronger depleted in ${ }^{13} \mathrm{C}$ compared to rhizotrons without beech trees indicating SOM decomposition. Specific respiration was increased by beech roots presumably due to root exudates lowering soil pH. Low soil pH decreased carbon use efficiency of bacteria leading to increased respiration and carbon loss from soil. In beech rhizotrons, only low amounts of litterderived carbon and nitrogen reached higher trophic levels. Ash had no effect on soil and microbial parameters but was more efficient in $\mathrm{N}$ uptake. The two tree species are suggested to peruse very different strategies in influencing the soil environment with beech influencing the soil mainly by root exudation and ash by litter input.

Chapter 4 focuses on the interactive effects of elevated $\mathrm{CO}_{2}$, nitrogen addition and reduced precipitation on soil biota in diverse grassland on sandy soil using the BioCON experiment. The response of microorganisms, protozoa, nematodes and soil microarthropods to the global change agents was investigated to identify changes in the belowground community. Elevated $\mathrm{CO}_{2}$ increased microbial biomass and the density of cil iates, microarthropod detritivores, and gamasid mites likely due to increased rhizodeposition. Taxa richness of soil microarthropods were detrimentally affected by nitrogen addition. Reduced precipitation decreased belowground herbivores and ciliates but had generally minor effects as the soil fauna is probably adapted to drought in this sandy soil. Int eractions of $\mathrm{CO}_{2}$ and $\mathrm{N}$ indicated positive bottom-up effects of elevated $\mathrm{CO}_{2}$ compensating for negative effects of nitrogen addition, but decreased nematode taxa richness. Interactive global change agents are suggested to result in taxonomically and functionally altered soil comm unities, e.g., simplification of the soil food web which likely negatively affects ecosystem functioning.

In Chapter 5, functional guilds of nematodes and nematode indices were used to specify changes in soil processes induced by global change agents in the BioCON experiment. Changes in the microbial community pointed to increased importance of the fungal decomposition channel. Interactions of elevated $\mathrm{CO}_{2}$ and nitrogen addition suggested changes in the fungal community and allowed to relate $\mathrm{Fu}_{2}$ to saprotrophic fungi and $\mathrm{Fu}_{4}$ to $\mathrm{AM}$ fungi. Root-feeding Longidoridae decreased at high $\mathrm{CO}_{2}$ and $\mathrm{N}$ levels suggesting improved plant performance and, therefore, a lower susceptibility to herbivores. El and SI indicated reduced food web stability at high nitrogen levels supporting the negative effect of nitrogen found in Chapter 4 . Reduced 
precipitation was of minor importance probably due to well adaption of nematodes to drought. This study highlights that global change a gents interactively affect soil processes. The importance of the fungal decomposition channel also points to an increase in recalcitrant compounds contributing to $C$ sequestration and sugge st a negative feedback to atmospheric $\mathrm{CO}_{2}$ concentrations.

Chapter 6 introduces NanoSIMS as a new method to obtain isotopic signatures of single nematodes aiming at integrating nematodes into food web analysis. The suitability of embedding techniques used for transmission electron microscopy were tested for measurement in high vacuum. First results are encouraging by providing maps on the distribution of different isotopes within the nemat ode body. In addition, fields of application are outlined.

\section{References}

Adair, E., Reich, P. B., Hobbie, S. E. \& Knops, J. M. H., 2009. Interactive Effects of Time, $\mathrm{CO}_{2}$, N, and Diversity on Total Belowground Carbon Allocation and Ecosystem Carbon Storage in a Grassland Community. Ecosystems 12, 1037-1052.

Ainsworth, E. A. \& Long, S. P., 2005. What have we learned from 15 years of free-air $\mathrm{CO}_{2}$ enrichment (FACE)? A meta-analytic review of the responses of photosynthesis, canopy properties and plant production to rising $\mathrm{CO}_{2}$. New Phytologist 165, 351-371.

Blankinship, J. C., Niklaus, P. A. \& Hungate, B. A., 2011. A meta-analysis of responses of soil biota to global change. Oecologia $165,553-65$.

Blaxter, M., 1998. Caenorhabditis elegans is a nematode. Science 282, 2041-2046.

Bongers, T., 1990. The maturity index: an ecological measure of environmental disturbance based on nematode species composition. Oecologia 83, 14-19.

Bongers, T. \& Ferris, H., 1999. Nematode community structure as a bioindicator in environmental monitoring. Trends in Ecology \& Evolution 14, 224-228.

Carney, K. M., Hungate, B. A., Drake, B. G. \& Megonigal, J. P., 2007. Altered soil microbial community at elevated $\mathrm{CO}_{2}$ leads to loss of soil carbon. Proceedings of the National Academy of Sciences of the United States of America 104, 4990-4995.

Adair C. E., Reich, P. B., Hobbie, S. E. \& Knops, J. M. H., 2009. Interactive effects of time, $\mathrm{CO}_{2}$, $\mathrm{N}$, and diversity on total belowground carbon Allocation and Ecosystem Carbon Storage in a Grassland Community. Ecosystems 12, 1037-1052. 
Clark, C. M. \& Tilman, D., 2008. Loss of plant species after chronic low-level nitrogen deposition to prairie grasslands. Nature $451,712-5$.

Cohen, J. E., 1989. Food webs and community structure. Perspectives in Ecological Theory (J. Roughgarden, R. M. May \& S. E. Levin, eds): pp. 181-202. University Press, Princeton, New York.

Dijkstra, F. A., Hobbie, S. E., Reich, P. B. \& Knops, J. M. H., 2005. Divergent effects of elevated $\mathrm{CO}_{2}, \mathrm{~N}$ fertilization, and plant diversity on soil $\mathrm{C}$ and $\mathrm{N}$ dynamics in a grassland field experiment. Plant and Soil 272, 41-52.

Farrar, J., Hawes, M., Jones, D. \& Lindow, S., 2003. How roots control the flux of carbon to the rhizosphere. Ecological Society of America 84, 827-837.

Ferris, H., Bongers, T. \& de Goede, R. G. M., 2001. A framework for soil food web diagnostics: extension of the nematode faunal analysis concept. Applied Soil Ecology 18, 13-29.

Gessner, M. O., Swan, C. M., Dang, C. K., McKie, B. G., Bardgett, R. D., Wall, D. H. \& Hättenschwiler, S., 2010. Diversity meets decomposition. Trends in Ecology \& Evolution 25, 372-380.

Grayston, S. J., Vaughan, D. \& Jones, D., 1996. Rhizosphere carbon flow in trees, in comparison with annual plants: the importance of root exudatio $n$ and its impact on microbial activity and nutrient availability. Applied Soil Ecology 5, 29-56.

Gregory, P. J., 2006. Roots, rhizosphere and soil: the route to a better understanding of soil science? European Journal of Soil Science 57, 2-12.

He, Z., Xu, M., Deng, Y., Kang, S., Kellogg, L., Wu, L., Van Nostrand, J. D., Hobbie, S. E., Reich, P. B. \& Zhou, J., 2010. Metagenomic analysis reveals a marked divergence in the structure of belowground microbial communities at elevated $\mathrm{CO}_{2}$. Ecology Letters 13: 564-575.

Hu, S., Chapin, F. S., Firestone, M. K., Field, C. B. \& Chiariello, N. R., 2001. Nitrogen limitation of microbial decomposition in a grassland under ele vated $\mathrm{CO}_{2}$. Nature $409,188-191$.

Hättenschwiler, S., Tiunov, A. V. \& Scheu, S., 2005. Biodiversity and litter decomposition in terrestrial ecosystems. Annual Review of Ecology, Evolution, and Systematics 36, 191218.

Högberg, M. N. \& Högberg, P., 2002. Extramatrical ectomycorrhizal mycelium contributes onethird of microbial biomass and produces, together with associated roots, half the dissolved organic carbon in a forest soil. New Phyt ologist 154, 791-795.

Högberg, M. N., Briones, M. J. I., Keel, S. G., Metcalfe, D. B., Campbell, C., Midwood, A. J., Thornton, B., Hurry, V., Linder, S., Näsholm, T. \& Högberg, P., 2010. Quantification of effects of season and nitrogen supply on tree below-ground carbon transfer to ectomycorrhizal fungi and other soil organisms in a boreal pine forest. New Phytologist 187, 485-493. 
IPCC, 2007. Climate Change 2007: The Physical Science Basis. Contribution of Working Group I to the Fourth Assessment Report of the Intergovernmental Panel on Climate Change (S Solomon, D Qin, M Manning, Z Chen, M Marquis, KB Averyt, M Tignor, and HL Miller, eds.). Cambridge University Press, Cambridge, United Kingdom and New York, USA.

Indermühle, A., Stocker, T. F., Joos, F., Fischer, H., Smith, H. J., Wahlen, M., Deck, B., Mastroianni, D., van der Werf, G. R., Blunier, T., Meyer, R. \& Stauffer, B., 1999. Holocene carbon-cycle dynamics based on $\mathrm{CO}_{2}$ trapped in ice at Taylor Dome, Antarctica. Nature 398, 121-126.

Jacob, M., Viedenz, K., Polle, A. \& Thomas, F. M., 2010. Leaf litter decomposition in temperate deciduous forest stands with a decreasing fraction of beech (Fagus sylvatica). Oecologia 164, 1083-1094.

Jacob, M., Weland, N., Platner, C., Schaefer, M., Leuschner, C. \& Thomas, F. M., 2009. Nutrient release from decomposing leaf litter of temperate deciduous forest trees along a gradient of increasing tree species diversity. Soil Biology and Biochemistry 41, 2122-2130.

Jones, D. L., Hodge, A. \& Kuzyakov, Y., 2004. Plant and mycorrhizal regulation of rhizodeposition. New Phytologist 163, 459-480.

Koranda, M., Schnecker, J., Kaiser, C., Fuchslueger, L., Kitzler, B., Stange, C. F., Sessitsch, A., Zechmeister-Boltenstern, S. \& Richter, A., 2011. Microbial processes and community composition in the rhizosphere of European beech - The influence of plant $C$ exudates. Soil Biology and Biochemistry 43, 551-558.

Kuzyakov, Y., 2010. Priming effects: Interactions between living and dead organic matter. Soil Biology and Biochemistry 42, 1363-1371.

Körner, C., 2000. Biosphere responses to $\mathrm{CO}_{2}$ enrichment. Ecological Applications 10, 15901619.

Langenbruch, C., Helfrich, M. \& Flessa, H., 2012. Effects of beech (Fagus sylvatica), ash (Fraxinus excelsior) and lime (Tilia spec.) on soil chemical properties in a mixed deci duous forest. Plant and Soil 352, 389-403.

Martinez, N. D., 1992. Constant connectance in community food webs. American Naturalist $139,1208-1218$.

McKinley, D. C., Ryan, M. G., Birdsey, R. A., Giardina, C. P., Harmon, M. E., Heath, L. S., Houghton, R. A., Jackson, R. B., Morrison, J. F., Murray, B. C., Pataki, D. E. \& Skog, K. E., 2011. A synthesis of current knowledge on forests and carbon storage in the United States. Ecological Applications 21, 1902-1924.

Moore, J. C. \& Hunt, H. W., 1988. Resource compartmentation and the stability of real ecosystems. Nature 333, 261-263. 
Paterson, E., Gebbing, T., Abel, C., Sim, A. \& Telfer, G., 2007. Rhizodeposition shapes rhizosphere microbial community structure in organic soil. New Phytologist $173,600-$ 610.

Paterson, E., Midwood, A. J. \& Millard, P., 2009. Through the eye of the needle: a review of isotope approaches to quantify microbial processes mediating soil carbon balance. New Phytologist 184, 19-33.

Paterson, E., Osler, G., Dawson, L. A., Gebbing, T., Sim, A. \& Ord, B., 2008. Labile and recalcitrant plant fractions are utilised by distinct microbial communities in soil: Independent of the presence of roots and mycorrhizal fungi. Soil Biology and Biochemistry 40, 1103-1113.

Paterson, E., Sim, A., Osborne, S. M. \& Murray, P. J., 2011. Long-term exclusion of plant-inputs to soil reduces the functional capacity of microbia I communities to mineralise recalcitrant root-derived carbon sources. Soil Biology and Bioch emistry 43, 1873-1880.

Pollierer, M. M., Langel, R., Körner, C., Maraun, M. \& Scheu, S., 2007. The underestimated importance of belowground carbon input for forest soil animal food webs. Ecology Letters 10, 729-736.

Pollierer, M. M., Langel, R., Scheu, S. \& Maraun, M., 2009. Compartmentalization of the soil animal food web as indicated by dual analysis of st able isotope ratios $\left({ }^{15} \mathrm{~N} /{ }^{14} \mathrm{~N}\right.$ and ${ }^{13} \mathrm{C} /{ }^{12} \mathrm{C}$ ). Soil Biology and Biochemistry $41,1221-1226$.

Reich, P. B., 2009. Elevated $\mathrm{CO}_{2}$ reduces losses of plant diversity caused by nitrogen deposition. Science 326, 1399-1402.

Reich, P. B., Hobbie, S. E., Lee, T., Ellsworth, D. S., West, J. B., Tilman, D., Knops, J. M. H., Naeem, S. \& Trost, J., 2006. Nitrogen limitation constrains sustainability of ecosystem response to $\mathrm{CO}_{2}$. Nature 440, 922-925.

Reich, P. B., Knops, J., Tilman, D., Craine, J., Ellsworth, D., Tjoelker, M., Lee, T., Wedin, D., Naeem, S., Bahauddin, D., Hendrey, G., Jose, S., Wrage, K., Goth, J. \& Bengston, W., 2001. Plant diversity enhances ecosystem responses to elevated $\mathrm{CO}_{2}$ and nitrogen deposition. Nature 411, 809-812.

Reich, P. B., Oleksyn, J., Modrzynski, J., Mrozinski, P., Hobbie, S. E., Eissenstat, D. M., Chorover, J., Chadwick, O. A., Hale, C. M. \& Tjoelker, M. G., 2005. Linking litter calcium, earthworms and soil properties: a common garden test with 14 tree species. Ecology Letters 8, 811818.

Scheu, S. \& Setälä, H., 2002. Multitrophic interactions in decomposer food-webs. Multitrophic Level Interactions (T. Tscharntke \& B. A. Hawkins, eds): pp. 233-264. Cambridge University Press, Cambridge.

Schulze, E. D. \& Freibauer, A., 2005. Carbon unlocked from soils. Nature 437, 11-12.

Smith, S. \& Read, D. J., 2008. Mycorrhizal symbiosis. Cambridge, UK, Academic Press. 
Swift, M. J., Heal, O. W. \& Anderson, J. M., 1979. Decomposition in terrestrial ecosystems. University of California Press, Berkeley.

Söderberg, K. H., Probanza, A., Jumpponen, A. \& Bååth, E., 2004. The microbial community in the rhizosphere determined by community-level physiological profiles (CLPP) and direct soil- and cfu-PLFA techniques. Applied Soil Ecology 25, 135-145

Wallander, H., Nilsson, L. O., Hagerberg, D. \& Bååth, E., 2001. Estimation of the biomass and seasonal growth of external mycelium of ectomycorrhizal fungi in the field. New Phytologist 151, 753-760.

Wardle, D. A., Verhoef, H. A. \& Clarholm, M., 1998. Trophic relationships in the soil microfoodweb: predicting the responses to a changing global. Global Change Biology 4, 713-727.

Yeates, G. W., 1999. Effects of plants on nematode community structure. Annual Review of Phytopathology 37, 127-149.

Yeates, G. W., Bongers, T., de Goede, R. G. M., Freckman, D. W. \& Georgieva, S. S., 1993. Feeding habits in soil nematode families and genera - an outline for soil ecologist. Journal of Nematology 25, 315-331.

Zak, D. R., Pregitzer, K. S., Curtis, P. S., Teeri, J. A., Fogel, R. \& Randlett, D. L., 1993. Elevated atmospheric $\mathrm{CO}_{2}$ and feedback between carbon and nitrogen cycles. Plant and Soil 151, 105-117. 


\section{| Chapter 2}

Tree species diversity versus tree species identity: Driving forces in structuring forest food webs as indicated by soil nematodes

Simone Cesarz, Liliane Ruess, Mascha Jacob, Andreas Jacob, Matthias Schaefer and Stefan Scheu

Published in Soil Biology and Biochemistry doi: 10.1016/j.soilbio.2013.02.020

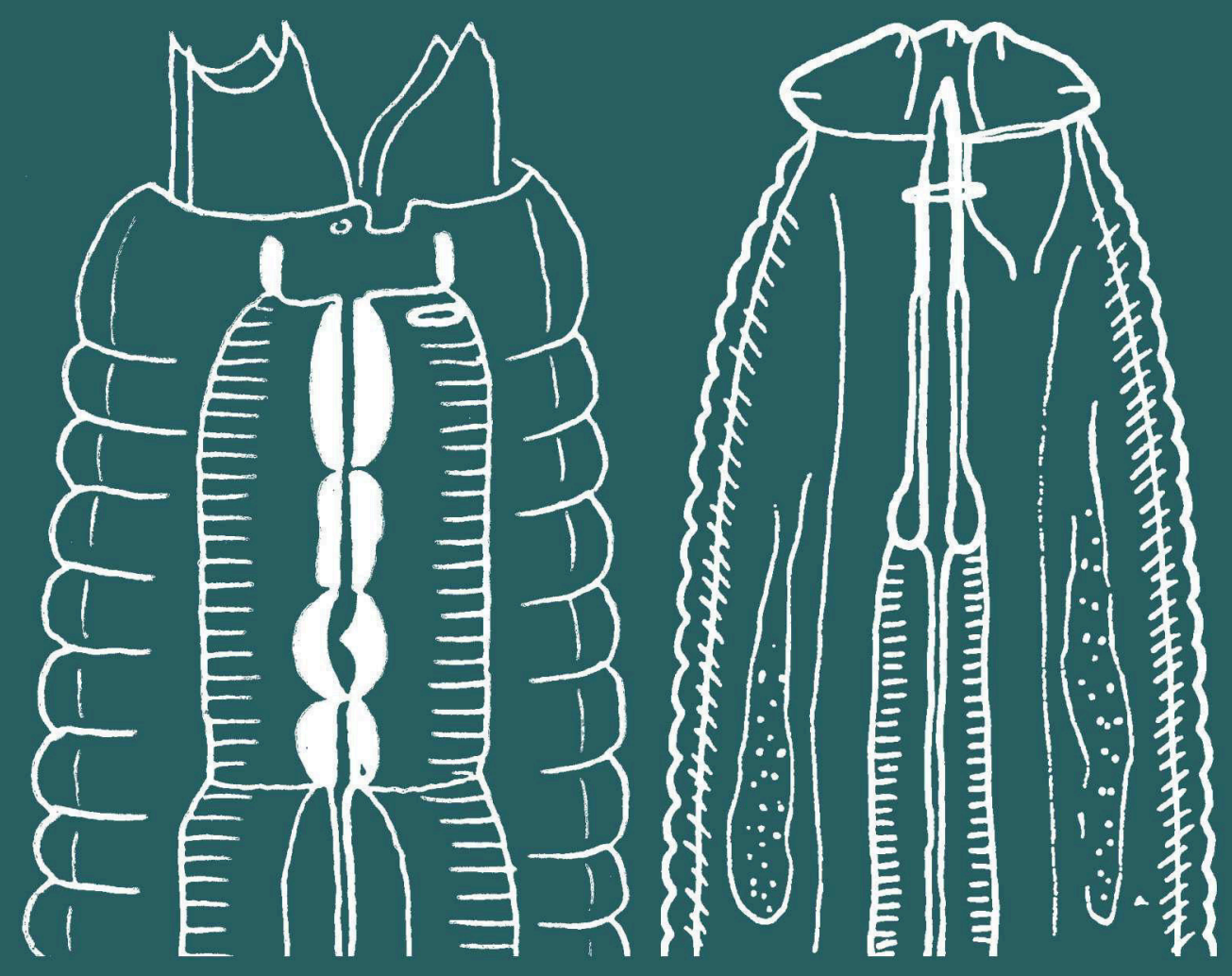




\section{Abstract}

Positive relationships between biodiversity and ecosystem functioning are reported in many studies. The underlying mechanisms are, however, only little understood, likely due to the focus on the aboveground system and neglecting species-specific traits. We used different clusters of tree species composition to investigate how tree species diversity and tree species identity structure affect belowground nematode communities. Since soil nematodes comprise different trophic groups and are strongly linked to the microbial community, results can provide insight on how soil food webs are structured. In addition, data on leaf litter and fine root biomass were included to account for different effects of aboveground and belowground resources. Clusters of three trees of one, two and three species were established within a temperate deciduous forest. Target tree species were European beech (Fagus sylvatica), common ash (Fraxinus excelsior) and lime (Tilia cordata, T. platyphyllos) differing in physiology, leaf litter quality and type of mycorrhiza. Tree species identity strongly affected nematode trophic structure, whereas tree species diversity had no impact. Ash beneficially affected bacterial-feeding nematodes, whereas fungal feeders were suppressed, likely caused by ash litter increasing soil $\mathrm{pH}$. Fostering of the bacterial food chain by ash additionally could be related to rhizodeposition gaining importance after disappearance of high quality ash litter in spring, highlighting seasonal shifts in root and leaf litter derived resources. The negative effect of ash on fungal-feeding nematodes is suggested to be due to the lack of ectomycorrhizal fungi as ash roots only form arbuscular mycorrhiza. In contrast, beech benefited fungal feeders and detrimentally affected bacterial feeders due to more acidic soil conditions that increase the competitive strength of fungi. Lime tended to negatively impact total nematode density but positively influenced plant-feeding nematodes. Generally, beech 
and ash strongly but opposingly influenced the trop hic structure of nematode communities suggesting that changes in tree species identity result in major shifts in the channeling of energy through decomposer food webs. The results indicate that the structure of soil food webs varies markedly with tree species and point to the importance of basal resources, i.e., leaf litter and rhizodeposits. This suggests bottom-up forces mediated by individual tree species to control major decomposition pathways rather than tree diversity.

\section{Introduction}

The relationship between biodiversity and ecosystem functioning has been mainly investigated considering aboveground systems. However, aboveground and belowground systems are intimately linked and a combined approach is of significant importance when aiming to understand ecosystem functioning (Wardle et al., 2004). Recently, above- and belowground systems were studied together in grasslands showing significant effects of biodiversity on the decomposer fauna (Scherber et al., 2010; Eisenhauer et al., 2012) being the driver of important ecosystem functions such as decomposition and nutrient mineralization. Grasslands, however, comprise short-lived plant species, therefore results are unlikely to be conferrable to forest systems, which, however, store about $60 \%$ of terrestrial carbon and are important for global carbon cycling (McKinley et al., 2011).

There is evidence that an increase in tree species diversity fosters ecosystem functions such as mineralization and decomposition (Morin et al., 2011). However, an increasing number of studies also report identity effects of single tree species to be stronger than tree species diversity (De Deyn et al., 2004; Nadrowski et al., 2010). It is therefore important to identify 
the species-specific mechanisms responsible for biodiversity effects. Thus, is the observed positive biodiversity effect due to an increase in species number (true biodiversity effect) or to individual tree species in mixtures (identity effect)? Regarding soil decomposer systems, differences between tree species result in differences in the resources provided mainly leaf litter and root exudation. Traditionally, effects of biodiversity on the decomposer system were investigated focusing on leaf litter residues regarded as the main driving factor for soil processes (Reich et al., 2005; Ball et al., 2009; Langenbruch et al., 2012) as it influences soil $\mathrm{pH}$, microbial activity and biomass (Swift et al., 1979; Wardle et al., 1998). Recently, the importance of root derived resources for soil food webs has been stressed (Albers et al., 2006; Pollierer et al., 2007; Endlweber et al., 2009; Keith et al., 2009). Rhizodeposits influence the soil system significantly by supplying labile resou rces to the food web and priming soil organic matter decomposition (Kuzyakov, 2010; Bird et al., 2011). In forest soils the fungal energy channel is of predominant importance as litter resources entering the soil are low in nitrogen and rich in lignin as compared to e.g., grassland soils (Wardle, 2002).

The quality of leaf litter and rhizodeposits (e.g., sugars, amino acids, organic acids) varies strongly between plant species (Grayston et al., 1996; Jones et al., 2004, 2009) and this likely differentially affects decomposers. High quality le af litter is characterized by low carbon-tonitrogen ratio, high quantities of soluble nutrients and high calcium and magnesium concentrations resulting in fast decomposition, whe reas high carbon-to-nitrogen ratio and high lignin content is regarded as low quality decr easing decomposition rates (Coûteaux et al., 1995; Cadish and Giller, 1997; Jacob et al., 2009). Rhizodeposits comprise mainly labile compounds so that quality differences may arise in specific compounds and the amount exudated (Grayston et al., 1996). Likely due to methodological limitation only few data are 
available on rhizodeposition of different tree species in soil (but see Scandellari et al., 2010; Fender et al., 2013) and no published work provide data on the effect of tree diversity on rhizodeposition.

We investigated the effect of tree species identity and tree species diversity in a diverse oldgrowth deciduous forest in Central Germany by identifying tree clusters of three individuals varying in tree species composition, comprising one, two or three species. Target tree species were European beech (Fagus sylvatica L.), common ash (Fraxinus excelsoir L.) and lime (Tilia cordata P. Mill., T. platyphyllos Scop). Leaf litter of beech has high carbon-to-nit rogen ratio and high lignin content while the opposite applies to leaf litter of ash and lime leaf litter, therefore spanning a wide range of litter quality (Jacob et al., 2009). Roots of beech and lime are colonized by ectomycorrhizal (EM) fungi, whereas ash roots are associated with arbuscular mycorrhizal (AM) fungi (Lang et al., 2011). Differences in rhizodeposition between the three tree species are little known. Fender et al., (2013) showed the amount of organic acids exudated by roots to be higher in beech as compared to ash, and Cesarz et al., (2013) suggested beech but not ash root exudates to acidify rhizosphere soil and to stimulate soil organic matter decomposition.

We used soil nematodes as representatives of soil invertebrates since they are highly abundant in soil, drive important ecosystem functions and comprise a wide range of trophic groups including bacteria, fungi and plant feeders as well as predators and omnivores (Yeates et al., 1993), therefore providing important information on how tree species structure soil food webs. There is a strong linkage between nematodes and their microbial diet, and the coincidence of functional groups of bacteria, fungi and their nematode grazers during 
succession provides the opportunity to monitor the abundance of available resources (Ruess and Ferris, 2004). The occurrence of nematode functional guilds provides information on general soil food webs conditions such as enrichment and structure (Ferris et al., 2001; Ruess and Ferris, 2004). Thus, nematodes are likely to respond to variations in resource diversity and due to their position in the soil food web, plant-feeding nematodes show the most pronounced association to plant communities (Yeates, 1999).

We hypothesized tree diversity to positively affect the abundance of soil nematodes due to an increase in resource partitioning and availability. Further we hypothesized tree species identity to affect specific nematode groups due to differences in litter and root quality, i.e., bacterial feeding nematodes to increase in ash and lime clusters due to high quality litter by favoring bacteria, whereas fungal-feeding nematodes to benefit from recalcitrant beech litter and acid rhizodeposits of beech roots favoring fung $i$.

\section{Material and methods}

\section{Site description}

The study site is located in the Hainich National Park, the largest cohesive deciduous forest in Central Germany $\left(51^{\circ} 06^{\prime} \mathrm{N}, 10^{\circ} 31^{\prime} \mathrm{E} ; 350 \mathrm{~m}\right.$ a.s.l). For the past four decades silvicultural management has been abandoned. Today large parts of the forest resemble semi-natural forest. Parent rock is Triassic Limestone covered by Pleistocene loess (Guckland et al., 2009). 


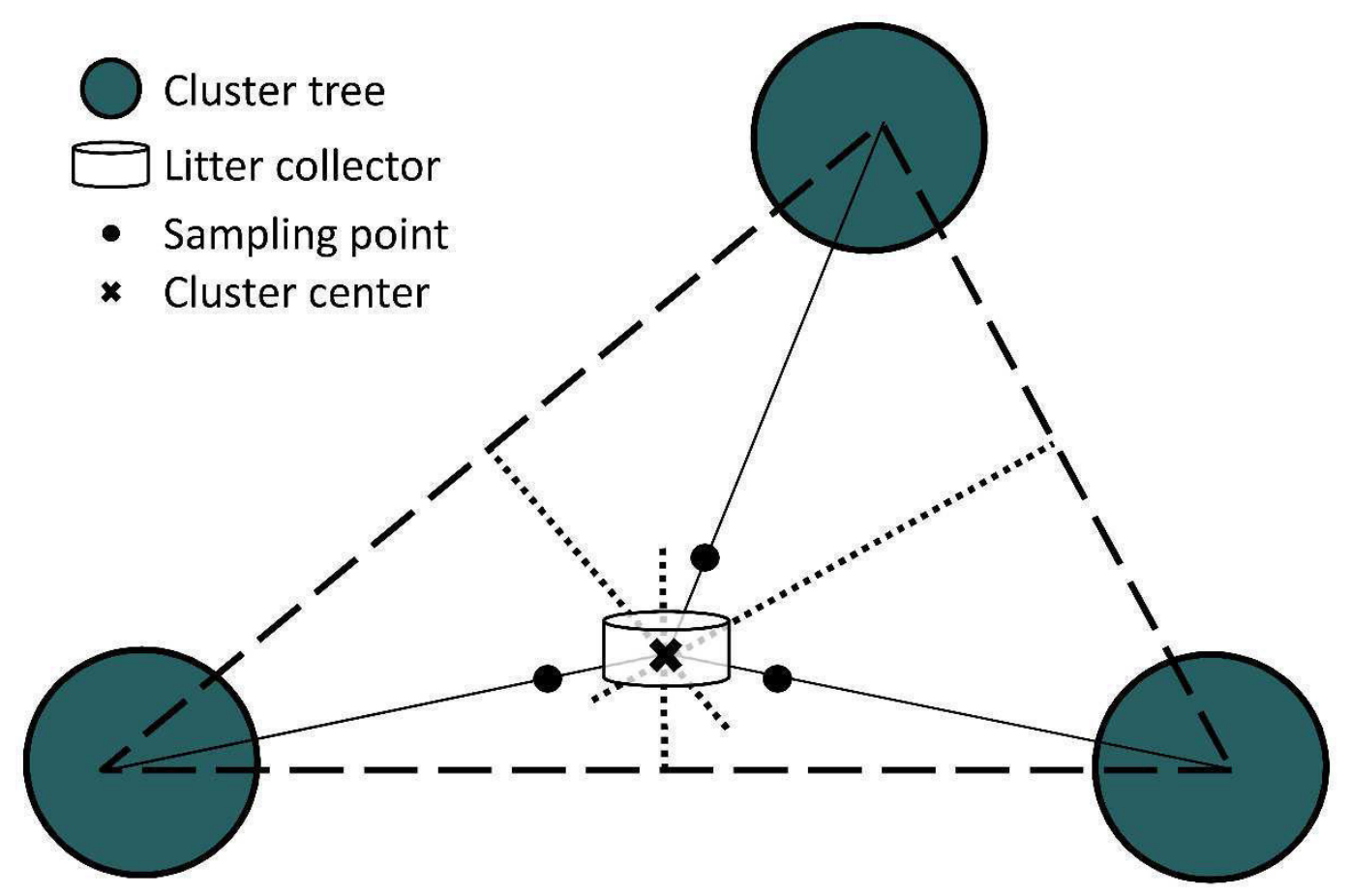

Figure 1 | Scheme of a tree cluster. Soil and fine root samples were taken $50 \mathrm{~cm}$ from the cluster center in direction to the cluster trees. Litter was collected with a litter collector in the cluster center.

The soil is characterized as Stagnic Luvisol with $\mathrm{pH}$ values ranging from $4.5 \pm 0.5$ to $5.8 \pm 0.6$. The mean annual temperature is $7.5^{\circ} \mathrm{C}$ and precipitation averages $590 \mathrm{~mm}$ year ${ }^{-1}$.

The three dominant tree species in the Hainich National Park are European beech ( $F$. sylvatica), common ash (F. excelsior) and lime (T.spp). To differentiate between tree species identity and diversity effects 28 tree clusters were established in areas with similar soil type. Individual clusters comprised three mature tree individuals of the canopy layer forming a triangle with the trees ranging 2.0 to $5.5 \mathrm{~m}$ from the center of the cluster (Fig. 1). Clusters were selected randomly and were spaced by at least 20 - 30 m (mean distance between clusters was $99 \mathrm{~m}$ ). This distance was accepted because earlier studies on tree fine root dynamics in this forest (e.g. Meinen et al., 2009a) had shown a maximum horizontal extension of the tree fine roots with $<15 \mathrm{~m}$, therefore water and nutrient fluxes between neighboring 
clusters could be excluded. Tree diameter at breast height was on average $34 \mathrm{~cm}$. No other trees or shrubs were present inside the clusters and cluster trees were at least $5 \mathrm{~m}$ away from trees outside the clusters. Single species clusters of beech (B), ash (A) and lime (L), two-species combinations, i.e., beech-ash (BA), beech-lime (BL) and ash-lime ( $A L)$, as well as the threespecies combination beech-ash-lime (BAL) were investigated. Each cluster was replicated four times.

\section{Nematode sampling}

In May 2008 three soil samples including the leaf litter layer $(2.5 \mathrm{~cm}$ ID; $5 \mathrm{~cm}$ depth) were taken in each cluster and pooled. Samples were taken $50 \mathrm{~cm}$ away from the cluster center towards the three cluster trees (Fig. 1). Pooled samples were stored at $4^{\circ} \mathrm{C}$ until extraction. Nematodes were extracted using a modified Baermann method (Ruess, 1995). Soil samples were placed in plastic vessels with gauze at the bottom coated with a milk filter, watered and kept at room temperature for $24 \mathrm{~h}$. Then, temperature was raised in steps of $5^{\circ} \mathrm{C}$ every hour until reaching $45^{\circ} \mathrm{C}$. Extracted nematodes were killed with $80^{\circ} \mathrm{C}$ hot water to achieve elongation of the animals and fixed by addition of formaldehyde solution (4\%). Animals were counted and a minimum of 100 individuals (or all if below), were identified and ascribed to trophic groups and functional guilds according to Yeates et al. (1993) and Ferris et al. (2001) using 100x magnification (Zeiss Axiovert 135, Jena, Germany). Nematode guilds comprise bacterivores $\left(\mathrm{Ba}_{\mathrm{x}}\right)$, fungivores $\left(\mathrm{Fu}_{\mathrm{x}}\right)$, plant-feeders $\left(\mathrm{PI}_{\mathrm{x}}\right)$ predators $\left(\mathrm{Pr}_{\mathrm{x}}\right)$ and omnivores $\left(\mathrm{Om}_{\mathrm{x}}\right)$ ranging along the $c-p$ scale from $x=1$ to $x=5$ (Bongers, 1990; Ferris et al., 2001). Nematodes of $c-p 1$ have a short life cycle, high fecundity, are tolerant to disturbance and can be ascribed to $r$-strategists. In contrast, nematodes of $c-p 5$ produce few large eggs, have a long life cycle 
combined with a long generation time and are sensitive to disturbance resembling $K$ strategists. Numbers between 1 and 5 reflect gradations between these opposing life history strategies (Bongers, 1990; Neher and Darby, 2009).

\section{Leaf litter and fine root sampling}

In the center of each cluster leaf litter was collected in 2008 on October 1 and 23, November 12, and in 2009 on March 10 using $35 \mathrm{~L}$ buckets with an aperture of $0.29 \mathrm{~m}^{2}$ (Fig. 1). Water could drain from the buckets through $8 \mathrm{~mm}$ holes in the bottom. Leaf litter was separated and assigned to the different tree species, oven-dried at $70^{\circ} \mathrm{C}$ for $48 \mathrm{~h}$ and weighed.

Fine root sampling was conducted in May 2008 in each of the clusters. Soil cores were taken from the upper $0-20 \mathrm{~cm}$ of the soil (including the organic layer) using a steel corer of a diameter of $35 \mathrm{~mm}$. Samples were transferred into polyethylene bags and stored at $4^{\circ} \mathrm{C}$ in darkness until processing within 3 weeks. The soil samples were soaked in water and sieved (mesh size $0.25 \mathrm{~mm}$ ) to separate fine roots ( $\leq 2 \mathrm{~mm}$ diameter) from soil. Coarse roots ( $\geq 2 \mathrm{~mm}$ diameter) and dead fine roots of trees, and roots of other vascular plants (mainly herbs and grasses) were discarded. Fine root fragments longer than $1 \mathrm{~cm}$ were collected manually using a pair of tweezers; living root fragments were ascribed to species using a stereomicroscope at 6-40x magnification. Species identification of fine roots present in the cluster was based on a set of morphological criteria, including branching patterns, color and surface structure of periderm, size and shape of root tips, diameter of fine and finest roots, established by Hölscher et al., (2002) and (Meinen et al., 2009b)Finally, fine root biomass was determined after drying at $70^{\circ} \mathrm{C}$ for $48 \mathrm{~h}$. Root mass was expressed as dry mass per square meter of soil surface area. 


\section{Statistical analysis}

Discriminant function analysis (DFA) was used to in vestigate if tree clusters in fact represented the respective leaf litter and fine root composition using STATISTICA 7.0 for Windows (StatSoft, Tulsa, USA, 2001). To analyze if the trophic structure of nematode communities varied with the composition of leaf litter and fine roots redundancy analysis (RDA) was performed. The length of gradient of detrended correspondance analysis (DCA) suggested linear to be more adequate than unimodal ordination (Ter Braak \& Šmilauer 2002). Analyses were implemented in CANOCO for Windows 4.5 (Wageningen, The Netherlands).

We used one-way ANOVA (SAS 9.2, SAS Institute Inc., Cary, NC, USA) to test the effects of tree species diversity (1, 2 and 3 tree species, respectively) and to observe differences in the ratio of fungal feeders-to-bacterial feeders within the different clusters (beech only (B), ash only (A), lime only (L) and all possible combinations, i .e., beech-ash (BA), beech-lime (BL), ash-lime $(A L)$ and beech-ash-lime (BAL)). Three-way ANOVA was used to inspect effects of beech (absence, B-; presence, $\mathrm{B}+$ ), ash (absence, A-; presence, $\mathrm{A}+$ ) and lime (absence, L-; presence, $L+)$ and all possible interactions. Akaike information criterion was calculated to improve the model by removing non-significant variables or inte ractions.

Multiple Regressions were calculated (SAS 9.2, SAS Institute Inc., Cary, NC, USA) to determine if the influence of tree species on nematodes was related to leaf litter or fine roots. We used stepwise regression analyses, combining forward and backward selection, to identify subsets of explanatory variables (Der and Everitt, 2002). Leaf litter and roots of tree species contributing only little to litter and fine root biomass $(<5 \%)$ were excluded, i.e., 
hornbeam (Carpinus betulus L.), oak (Quercus spec.) and maple (Acer pseudoplatanus L.). Nematode abundance data, leaf litter biomass and fine root biomass were log-transformed to meet requirements of ANOVA and regression analyses.

\section{Results}

\section{Leaf litter and fine roots}

Clusters of trees were well represented by their leaf litter and fine root composition as indicated by DFA (Fig. 2). Single species clusters of beech, ash and lime contained more species-specific leaf litter and fine root biomass than mixed clusters. The proportion of the corresponding litter and fine root biomass in pure clusters decreased with increasing tree species diversity. Fine roots had a lower spreading than leaf litter. In pure lime and pure ash clusters, high amounts of fine roots of beech were present. BAL clusters were in midst of the other treatments.

RDA indicated that the nematode community composition in clusters with beech differed significantly from that in other clusters, especially from clusters where ash co-occurred (Fig. 3). Beech clusters were associated with low amounts of leaf litter and fine roots of ash, and high amounts of lime litter. Mainly fungal feeders occurred in beech only clusters, whereas A, BA and BAL clusters were dominated by bacterial feeders. Generally, ash leaf litter and ash fine roots contributed most to the separation of the samples. 

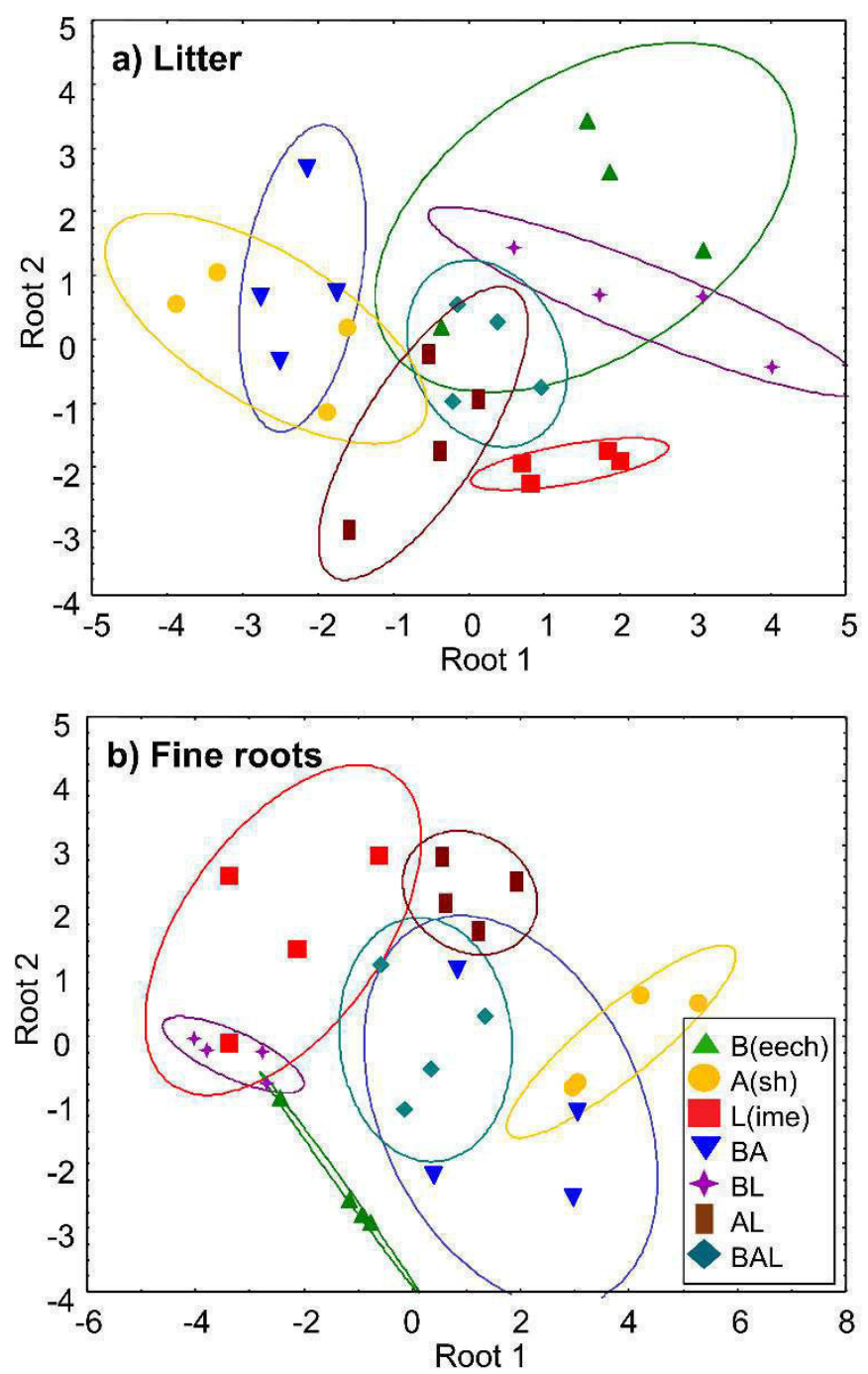

Figure 2 | Discriminant function analysis (DFA) of the composition of leaf litter (a) and fine roots (b) in tree clusters, i.e., the fraction of litter and fine roots of beech (B), ash (A) and lime (L). Ellipses rep resent confidence intervals at $P=0.05$; Wilks Lambda $=0.0435, F_{36,73}=2.11, P=0.0034$ and Wilks Lambda $=0.0158$, $F_{36,73}=3.19, P=0.0001$ for litter and fine roots, respectively.

\section{Tree species identity and diversity}

Ash beneficially affected bacterial feeders (Table 1); densities were significantly higher in clusters with ash only (i.e., B- and L- clusters; two-way interaction) and decreased when other tree species were present (Fig. 4a, Table 2). This also applied for all families of bacterial feeders and the respective functional guilds both reaching high densities in A+ clusters reflecting the overall beneficial effect of ash on bacterial-feeding nematodes (Table S1). 


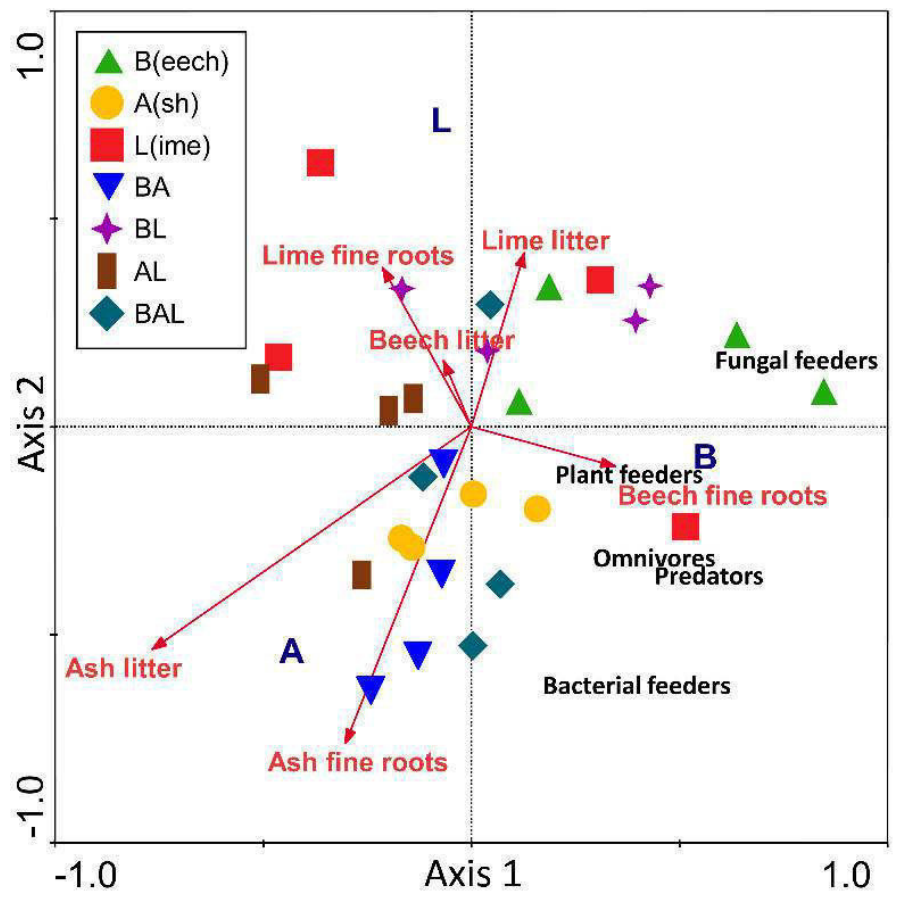

Figure 3 | Redundancy analysis (RDA) of nematode trophic groups in tree clusters. Environmental variables were litter and root biomasses $\left(\mathrm{g} \mathrm{m}^{-2}\right)$ of the target tree species beech, ash and lime (red arrows). Nominal variables are indicated by bold capital letters ( $B$, beech; $A$, ash; L, lime). Eigenvalue of axis 1 and axis 2 is 0.256 and 0.069 , respectively $(F=7.20, P=0.0360$ and $F=1.81, P=0.0560)$, explaining $25.6 \%$ and $6.9 \%$ of the variation, respectively.

Opportunistic $\mathrm{Ba}_{1}$ nematodes largely followed this positive ash influence but were also abundant in the 3-species cluster (Fig. 4b). Other tree species did not influence bacterial feeders except densities of $\mathrm{Ba}_{3}$ nematodes which were significantly higher in $\mathrm{B}+$ clusters (Table 1).

In contrast, fungal-feeding nematodes were negatively affected by ash but benefitted from beech (Fig. 4c). Fu 4 nematodes made up $24 \pm 4 \%$ in beech only clusters but reached only low frequency of $5 \pm 2 \%$ in the other clusters. The positive effect of beech and the negative effect of ash are illustrated by the interaction of ash and lime, $\mathrm{Fu}_{4}$ nematodes increased significantly in clusters with beech only (i.e., the A- and L- clusters) and decreased when either ash and lime or both were present (Tables 1, 2). 

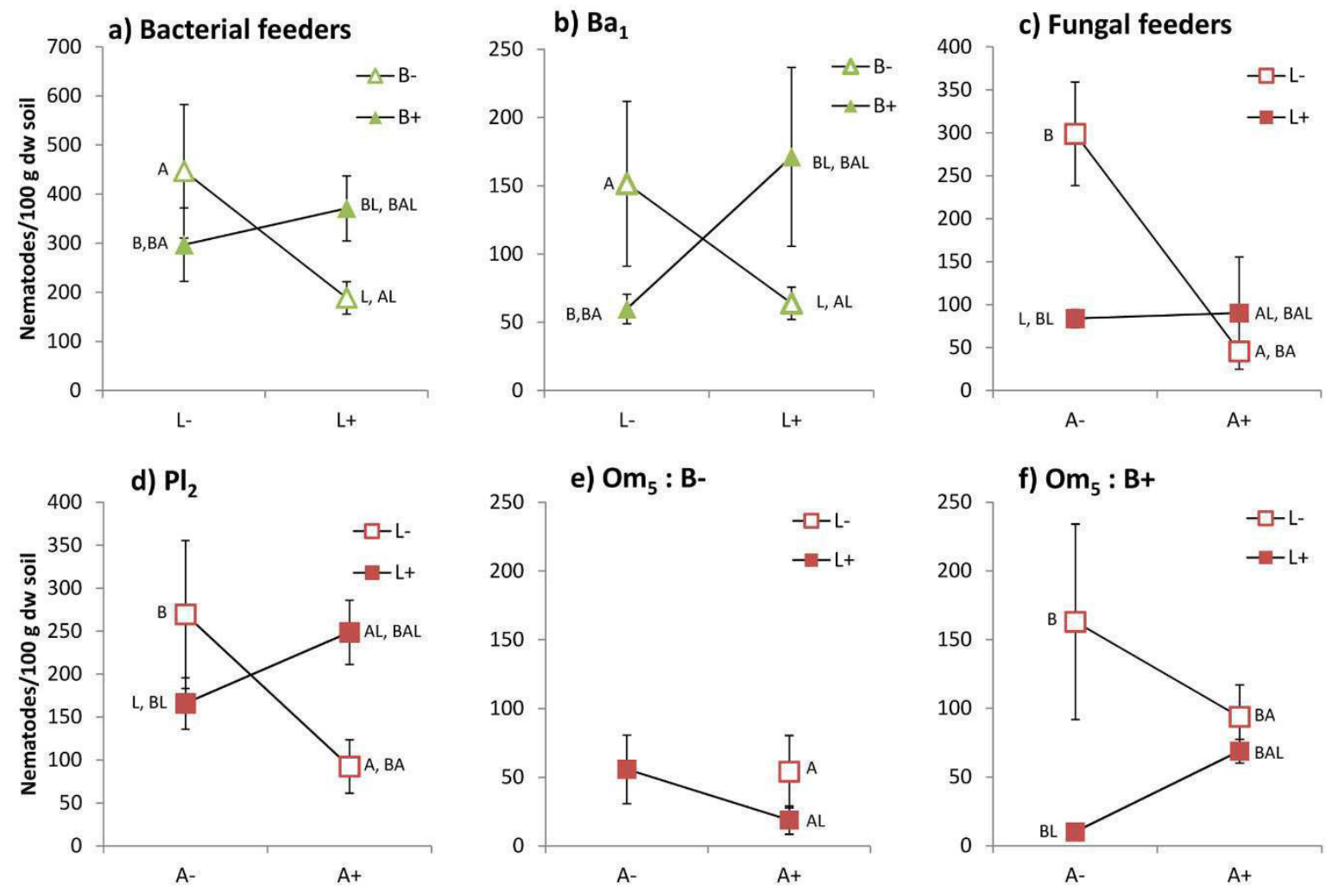

Figure 4 | Nematode abundances of trophic groups and function al guilds as affected by the interactions of beech ( $B-$, absent; $B+$, present), ash ( $A-$, absent; $A+$, present) and lime ( $L-$, absent; $L+$, present) in different tree clusters. Capitals in graphs indicate the different clusters. B: beech only, A: ash only, L: lime only, BA: beechash, BL: beech-lime, AL: ash lime, BAL: beech-ash-lime clusters. PI: plant feeders, Ba: bacterial feeders, Fu: fungal feeders, Om: omnivors, Pr: predators, subscript numbers indicate the corresponding $c-p$ classification according to Bongers (1990). Means \pm SE.

The functional guilds of plant feeders $\mathrm{Pl}_{2}$ and $\mathrm{Pl}_{3}$ differentially responded to tree species. Hoplolaimidae were dominant in $\mathrm{Pl}_{3}$ and were responsible for the positive influence of ash on this nematode guild (Tables $1, \mathrm{~S} 1$ ). In addition, th eir densities increased significantly when beech was present and lime absent. Tylenchidae were dominant in $\mathrm{Pl}_{2}$ and were largely responsible for the response of this nematode guild (Table S1). Densities of $\mathrm{Pl}_{2}$ nematodes were high in beech only clusters (i.e., A- and L- clusters) and declined significantly when ash was present $(-63 \%$, Tables 1, 2). This negative effect of ash was reduced by lime (Fig. 4d, Table 1). Generally, $\mathrm{Pl}_{2}$ nematodes benefited from the presence of lime, whereas most other nematodes tended to be negatively influenced by this tree species. Qudsianematidae were 
Table 1 | ANOVA table of $F$ - and $P$-values on the effects of beech, ash and lime presence and absence, respectively, and all possible interactions on the abundance of trophic groups and functional guilds (for families consider Table S1) of soil nematodes. Pl: plant fee ders, Ba: bacterial feeders, Fu: fungal feeders, Om: omnivores, Pr: predators, subscript numbers indicate the corresponding $c-p$ classification according to Bongers (1990). Rare groups are not displayed and were not analyzed with ANOVA due to very low densities (<2\%). For means and SE see Table 2.

\begin{tabular}{|c|c|c|c|c|c|c|c|c|c|c|c|c|c|c|c|c|c|}
\hline \multirow[b]{3}{*}{ Total } & \multicolumn{2}{|c|}{ B(eech) } & & \multicolumn{2}{|c|}{$A(s h)$} & & \multicolumn{2}{|c|}{ L(ime) } & & \multicolumn{2}{|c|}{$B \times A$} & \multicolumn{2}{|c|}{$B \times L$} & \multicolumn{2}{|c|}{$A \times L$} & \multicolumn{2}{|c|}{$B \times A \times L$} \\
\hline & $\boldsymbol{F}$ & $\boldsymbol{P}$ & & $\boldsymbol{F}$ & $P$ & & $\boldsymbol{F}$ & $P$ & & $\boldsymbol{F}$ & $P$ & $\boldsymbol{F}$ & $\boldsymbol{P}$ & $\boldsymbol{F}$ & $P$ & $\boldsymbol{F}$ & $\boldsymbol{P}$ \\
\hline & 3.90 & 0.0616 & & 0.50 & 0.49 & & 0.82 & 0.3755 & & 0.69 & 0.42 & 0.53 & 0.4730 & excl & ded & 1.23 & 0.2794 \\
\hline \multicolumn{18}{|l|}{ Trophic groups } \\
\hline Plant feeders & 1.22 & 0.2824 & & 0.14 & 0.7140 & & 0.44 & 0.5132 & & \multicolumn{2}{|c|}{ excluded } & 0.40 & 0.5364 & 2.01 & 0.1710 & 0.11 & 0.7425 \\
\hline Bacterial feeders & 2.08 & 0.1636 & & 6.54 & 0.0183 & $\uparrow$ & 1.21 & 0.2830 & & 1.72 & 0.2044 & 5.46 & 0.0295 & \multicolumn{2}{|c|}{ excluded } & 0.00 & 1.0000 \\
\hline Fungal feeders & 3.94 & 0.0602 & & 3.79 & 0.0651 & & 0.24 & 0.6324 & & 2.00 & 0.1717 & \multicolumn{2}{|c|}{ excluded } & 6.32 & 0.0202 & 0.00 & 1.0000 \\
\hline Predators & 3.15 & 0.0904 & & 0.02 & 0.8867 & & 3.54 & 0.0740 & & 1.21 & 0.2834 & 0.00 & 1.0000 & \multicolumn{2}{|c|}{ excluded } & 2.57 & 0.1242 \\
\hline Omnivores & 0.23 & 0.6364 & & 0.89 & 0.3561 & & 3.53 & 0.0744 & & \multicolumn{2}{|c|}{ excluded } & 1.05 & 0.3169 & 0.75 & 0.3961 & 1.20 & 0.2850 \\
\hline \multicolumn{18}{|l|}{ Functional guilds } \\
\hline $\mathrm{Pl}_{2}$ & 2.41 & 0.1353 & & 1.58 & 0.2225 & & 5.57 & 0.0280 & $\uparrow$ & \multicolumn{2}{|c|}{ excluded } & 1.63 & 0.2154 & 8.08 & 0.0097 & 0.00 & 1.0000 \\
\hline $\mathrm{Pl}_{3}$ & 1.28 & 0.2703 & & 4.81 & 0.0398 & $\uparrow$ & 2.35 & 0.1402 & & 0.41 & 0.5288 & \multicolumn{2}{|c|}{ excluded } & 0.00 & 1.0000 & 1.74 & 0.2016 \\
\hline $\mathrm{Ba}_{1}$ & 0.05 & 0.8182 & & 0.53 & 0.4764 & & 0.57 & 0.4582 & & \multicolumn{2}{|c|}{ excluded } & 4.47 & 0.0466 & 1.84 & 0.1894 & 2.78 & 0.1105 \\
\hline $\mathrm{Ba}_{2}$ & 1.41 & 0.2487 & & 3.59 & 0.0720 & & 1.18 & 0.2903 & & 1.79 & 0.1950 & \multicolumn{2}{|c|}{ excluded } & 0.00 & 1.0000 & 0.00 & 1.0000 \\
\hline $\mathrm{Ba}_{3}$ & 4.54 & 0.0450 & $\uparrow$ & 0.87 & 0.3606 & & 0.03 & 0.8674 & & 0.99 & 0.3307 & 0.15 & 0.7060 & \multicolumn{2}{|c|}{ excluded } & 1.75 & 0.1998 \\
\hline $\mathrm{Ba}_{4}$ & 1.23 & 0.2795 & & 0.56 & 0.4642 & & 1.00 & 0.3280 & & 3.81 & 0.0644 & 0.00 & 1.0000 & \multicolumn{2}{|c|}{ excluded } & 0.00 & 1.0000 \\
\hline $\mathrm{Fu}_{2}$ & 0.82 & 0.3742 & & 0.00 & 0.9976 & & 0.23 & 0.6348 & & 0.95 & 0.3406 & 2.91 & 0.1026 & \multicolumn{2}{|c|}{ excluded } & 0.09 & 0.7639 \\
\hline $\mathrm{Fu}_{4}$ & 9.62 & 0.0054 & $\uparrow$ & 12.40 & 0.0021 & $\downarrow$ & 1.66 & 0.2118 & & 0.00 & 1.0000 & \multicolumn{2}{|c|}{ excluded } & 10.00 & 0.0047 & 0.00 & 1.0000 \\
\hline $\mathrm{Pr}_{3}$ & 0.19 & 0.6666 & & 1.83 & 0.1906 & & 0.37 & 0.5503 & & 0.30 & 0.5915 & \multicolumn{2}{|c|}{ excluded } & 0.64 & 0.4339 & 1.79 & 0.1953 \\
\hline $\mathrm{Pr}_{4}$ & 0.03 & 0.8719 & & 1.39 & 0.2513 & & 1.40 & 0.2505 & & \multicolumn{2}{|c|}{ excluded } & 1.93 & 0.1792 & 0.58 & 0.4540 & 0.00 & 1.0000 \\
\hline $\mathrm{Om}_{4}$ & 0.04 & 0.8424 & & 0.54 & 0.4721 & & 0.42 & 0.5237 & & \multicolumn{2}{|c|}{ excluded } & 1.35 & 0.2583 & 1.53 & 0.2290 & 0.00 & 1.0000 \\
\hline $\mathrm{Om}_{5}$ & 4.30 & 0.0507 & & 0.10 & 0.7578 & & 11.98 & 0.0023 & $\downarrow$ & \multicolumn{2}{|c|}{ excluded } & 0.70 & 0.4126 & 3.47 & 0.0766 & 13.25 & 0.0015 \\
\hline
\end{tabular}

$\underset{\omega}{\omega}$ 
Table 2 | Means \pm SE of abundance (Individuals/g soil dry wei ght) of nematodes grouped in trophic groups and fun ctional guilds (for families see Table S2) as affected by beech (B), ash (A) and lime (L) presence (+) or absence (-), respectively. PI: plant feeders, Ba: bacterial feeders, Fu: fungal feeders, Om: omnivores, Pr: predators, subscript numbers indicate the corresponding $c-p$ classification according to Bongers (1990). * = not analyzed with ANOVA due to very low densities (<2\%). For statistical analysis see Table 1.

\begin{tabular}{|c|c|c|c|c|c|c|c|c|c|c|c|c|c|c|c|c|c|c|c|c|c|}
\hline \multirow[b]{6}{*}{ Total } & \multicolumn{9}{|c|}{$-B$} & \multicolumn{12}{|c|}{$+B$} \\
\hline & \multirow{2}{*}{\multicolumn{3}{|c|}{$\begin{array}{l}-\mathrm{A} \\
+\mathrm{L}\end{array}$}} & \multicolumn{6}{|c|}{$+A$} & \multicolumn{6}{|c|}{$-A$} & \multicolumn{6}{|c|}{$+A$} \\
\hline & & & & \multicolumn{3}{|c|}{$-L$} & \multicolumn{3}{|c|}{$+\mathrm{L}$} & \multicolumn{3}{|c|}{$-L$} & \multirow{2}{*}{\multicolumn{3}{|c|}{$\frac{+\mathrm{L}}{\text { Beech-Lime }}$}} & \multicolumn{3}{|c|}{$-L$} & \multicolumn{3}{|c|}{$+\mathrm{L}$} \\
\hline & \multicolumn{3}{|c|}{ Lime } & \multicolumn{3}{|c|}{ Ash } & \multicolumn{3}{|c|}{ Ash-Lime } & \multicolumn{3}{|c|}{ Beech } & & & & \multicolumn{3}{|c|}{ Beech-Ash } & \multicolumn{3}{|c|}{ Beech-Ash-Lime } \\
\hline & Mean & & SE & Mean & & SE & Mean & & SE & Mean & & SE & Mean & & SE & Mean & & SE & Mean & & SE \\
\hline & 532.43 & \pm & 140.56 & 910.83 & \pm & 279.09 & 570.23 & \pm & 71.52 & 1100.87 & \pm & 261.30 & 785.02 & \pm & 210.96 & 1060.92 & \pm & 497.30 & 1264.29 & \pm & 149.67 \\
\hline \multicolumn{22}{|l|}{ Trophic groups } \\
\hline Plant feeders & 175.65 & \pm & 38.54 & 252.85 & \pm & 127.22 & 223.88 & \pm & 38.76 & 296.28 & \pm & 90.39 & 234.62 & \pm & 74.66 & 365.60 & \pm & 266.19 & 462.85 & \pm & 98.48 \\
\hline Bacterial feeders & 176.10 & \pm & 63.78 & 446.81 & \pm & 136.17 & 201.54 & \pm & 30.34 & 210.76 & \pm & 28.61 & 245.51 & \pm & 42.67 & 383.71 & \pm & 143.16 & 496.91 & \pm & 89.55 \\
\hline Fungal feeders & 34.10 & \pm & 17.89 & 48.84 & \pm & 22.64 & 41.95 & \pm & 18.11 & 298.90 & \pm & 86.25 & 133.33 & \pm & 42.59 & 42.47 & \pm & 36.47 & 138.56 & \pm & 66.82 \\
\hline Predators & 105.38 & \pm & 33.07 & 99.06 & \pm & 23.08 & 53.68 & \pm & 20.48 & 193.54 & \pm & 72.14 & 99.12 & \pm & 53.76 & 158.44 & \pm & 45.52 & 139.97 & \pm & 35.14 \\
\hline Omnivores & 38.89 & \pm & 17.92 & 60.46 & \pm & 30.05 & 49.19 & \pm & 24.54 & 97.71 & \pm & 29.63 & 72.43 & \pm & 26.72 & 96.06 & \pm & 36.28 & 25.99 & \pm & 13.75 \\
\hline \multicolumn{22}{|l|}{ Functional guilds } \\
\hline $\mathrm{Pl}_{2}$ & 126.16 & \pm & 25.75 & 106.54 & \pm & 51.76 & 172.40 & \pm & 42.80 & 269.54 & \pm & 86.19 & 205.62 & \pm & 49.70 & 78.48 & \pm & 41.35 & 324.88 & \pm & 28.41 \\
\hline $\mathrm{Pl}_{3}$ & 49.49 & \pm & 20.95 & 146.32 & \pm & 75.83 & 51.48 & \pm & 11.35 & 26.74 & \pm & 6.87 & 29.00 & \pm & 26.50 & 287.12 & \pm & 224.88 & 137.97 & \pm & 90.59 \\
\hline $\mathrm{Ba}_{1}$ & 59.18 & \pm & 21.42 & 151.61 & \pm & 60.31 & 68.76 & \pm & 13.50 & 69.27 & \pm & 6.47 & 79.36 & \pm & 26.46 & 50.28 & \pm & 20.96 & 263.12 & \pm & 117.04 \\
\hline $\mathrm{Ba}_{2}$ & 76.48 & \pm & 48.33 & 220.91 & \pm & 106.12 & 77.35 & \pm & 12.48 & 74.16 & \pm & 12.43 & 80.23 & \pm & 11.89 & 195.95 & \pm & 112.10 & 148.58 & \pm & 29.43 \\
\hline $\mathrm{Ba}_{3}$ & 18.29 & \pm & 10.59 & 46.76 & \pm & 36.92 & 14.09 & \pm & 0.74 & 27.14 & \pm & 13.10 & 44.41 & \pm & 10.13 & 100.11 & \pm & 40.69 & 44.23 & \pm & 12.06 \\
\hline $\mathrm{Ba}_{4}$ & 22.16 & \pm & 16.02 & 27.53 & \pm & 6.66 & 41.33 & \pm & 17.79 & 40.19 & \pm & 17.59 & 41.51 & \pm & 13.43 & 37.38 & \pm & 6.49 & 40.98 & \pm & 19.50 \\
\hline $\mathrm{Fu}_{2}$ & 6.56 & \pm & 4.10 & 20.59 & \pm & 9.27 & 14.17 & \pm & 11.04 & 18.48 & \pm & 9.89 & 25.50 & \pm & 11.37 & 7.74 & \pm & 6.00 & 53.40 & \pm & 21.00 \\
\hline $\mathrm{Fu}_{3} *$ & 1.42 & \pm & 1.42 & 24.28 & \pm & 15.29 & 9.01 & \pm & 3.26 & 0.00 & \pm & 0.00 & 1.39 & \pm & 1.39 & 14.06 & \pm & 12.24 & 4.21 & \pm & 4.21 \\
\hline $\mathrm{Fu}_{4}$ & 26.12 & \pm & 12.62 & 3.97 & \pm & 3.97 & 18.77 & \pm & 7.21 & 280.41 & \pm & 81.47 & 106.44 & \pm & 46.72 & 20.67 & \pm & 18.46 & 80.95 & \pm & 49.19 \\
\hline $\mathrm{Pr}_{3}$ & 5.61 & \pm & 4.40 & 33.92 & \pm & 31.12 & 11.12 & \pm & 3.78 & 13.87 & \pm & 13.87 & 8.62 & \pm & 3.58 & 41.05 & \pm & 21.38 & 11.27 & \pm & 6.59 \\
\hline $\mathrm{Pr}_{4}$ & 33.28 & \pm & 13.68 & 26.53 & \pm & 13.63 & 38.07 & \pm & 23.31 & 76.10 & \pm & 28.90 & 63.81 & \pm & 27.47 & 55.01 & \pm & 32.43 & 14.72 & \pm & 8.07 \\
\hline $\mathrm{Om}_{4}$ & 49.65 & \pm & 24.14 & 45.03 & \pm & 6.91 & 34.82 & \pm & 11.12 & 38.35 & \pm & 18.80 & 89.03 & \pm & 52.42 & 64.69 & \pm & 34.64 & 71.14 & \pm & 26.87 \\
\hline $\mathrm{Om}_{5}$ & 55.72 & \pm & 24.93 & 54.03 & \pm & 26.34 & 18.86 & \pm & 10.25 & 162.94 & \pm & 71.11 & 10.09 & \pm & 2.05 & 93.75 & \pm & 23.34 & 68.83 & \pm & 8.62 \\
\hline
\end{tabular}


detrimentally affected by lime but the negative effect was reduced by beech (Tables S1, S2) and also $\mathrm{Om}_{5}$ nematodes decreased significantly in presence of lime but increased when beech was present (Fig. 4e, f). Predatory nematodes were not significantly affected by tree species.

Tree species diversity did not significantly affect total nematode abundance (Fig. 5, Table 3). However, nematode density was highest in three species clusters (Mean \pm SE: $1264 \pm 75$ ind. $100 \mathrm{~g}^{-1}$ soil dry weight) as compared to one (848 \pm 41 ind. $100 \mathrm{~g}^{-1}$ soil dry weight) and two species clusters (805 \pm 76 ind. $100 \mathrm{~g}^{-1}$ soil dry weight). Also none of the trophic groups or functional guilds studied was significantly influen ced by tree species diversity although densities were often highest in the high diverse clusters (Fig. 5).

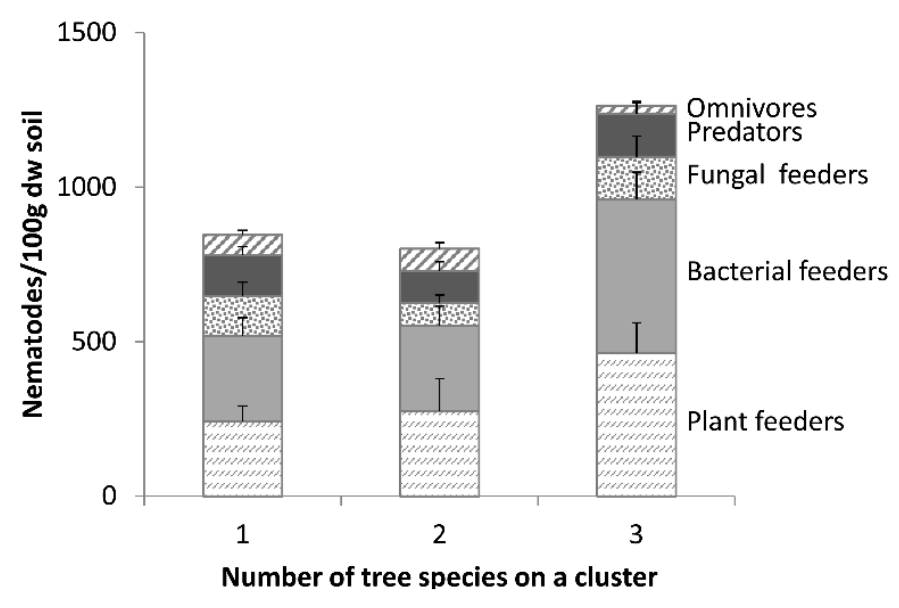

Figure 5 | Total and trophic nematode abundances as affected by tree species diversity. Means \pm SE.

\section{The role of above- vs. belowground plant input}

To explore whether tree species effects were due to above- or belowground impacts, we employed multiple regression analyses relating nematode densities to leaf litter and fine root 


\begin{tabular}{|c|c|c|}
\hline & $\boldsymbol{F}$ & $\boldsymbol{P}$ \\
\hline Total soil nematodes & 1.80 & 0.1868 \\
\hline \multicolumn{3}{|l|}{ Trophic groups } \\
\hline Plant feeders & 2.00 & 0.1564 \\
\hline Bacterial feeders & 2.24 & 0.1274 \\
\hline Fungal feeders & 0.40 & 0.6778 \\
\hline Predators & 1.17 & 0.3254 \\
\hline Omnivores & 2.67 & 0.0892 \\
\hline \multicolumn{3}{|l|}{ Functional guilds } \\
\hline $\mathrm{Pl}_{2}$ & 2.65 & 0.0907 \\
\hline $\mathrm{Pl}_{3}$ & 0.13 & 0.8818 \\
\hline $\mathrm{Ba}_{1}$ & 3.10 & 0.0625 \\
\hline $\mathrm{Ba}_{2}$ & 0.95 & 0.4007 \\
\hline $\mathrm{Ba}_{3}$ & 2.60 & 0.0939 \\
\hline $\mathrm{Ba}_{4}$ & 1.16 & 0.3312 \\
\hline $\mathrm{Fu}_{2}$ & 0.87 & 0.4295 \\
\hline $\mathrm{Fu}_{4}$ & 0.45 & 0.6411 \\
\hline $\mathrm{Pr}_{3}$ & 1.47 & 0.2500 \\
\hline $\operatorname{Pr}_{4}$ & 1.94 & 0.1642 \\
\hline $\mathrm{Om}_{4}$ & 0.67 & 0.5190 \\
\hline $\mathrm{Om}_{5}$ & 2.94 & 0.0715 \\
\hline
\end{tabular}

Table 3 | ANOVA table of $F$ - and $P$-values on the effects of tree species diversity $(1,2$ and 3$)$ on the abundance of total soil nematodes and of trophic groups and functional guilds of soil nematodes. PI: plant feeders, Ba: bacterial feeders, Fu: fungal feeders, Om: omnivors, Pr: predators, subscript numbers indicate the corresponding $c-p$ classification according to Bongers (1990). Rare groups are not displayed and were not analyzed with ANOVA due to low densities $(<2 \%)$.

\begin{tabular}{|c|c|c|c|c|}
\hline & \multirow[b]{2}{*}{ Tree species } & \multicolumn{3}{|c|}{ Litter biomass } \\
\hline & & $\boldsymbol{F}$ & $\boldsymbol{P}$ & $R^{2}$ \\
\hline $\mathrm{Pl}_{2}$ & B & 10.02 & 0.0040 & 0.2338 \\
\hline Tylenchidae $\left(\mathrm{Pl}_{2}\right)$ & B & 7.52 & 0.0109 & 0.2244 \\
\hline Fungal feeders & A & 13.56 & 0.0011 & 0.3427 \\
\hline \multirow[t]{3}{*}{$\mathrm{Fu}_{4}$} & B & 8.28 & 0.0079 & 0.2416 \\
\hline & & \multicolumn{3}{|c|}{ Root biomass } \\
\hline & Tree species & $\boldsymbol{F}$ & $\boldsymbol{P}$ & $R^{2}$ \\
\hline Bacterial feeders & A & 5.59 & 0.0258 & 0.1770 \\
\hline Rhabditidae $\left(\mathrm{Ba}_{1}\right)$ & $\mathbf{L}$ & 6.36 & 0.0181 & 0.1966 \\
\hline Mononchidae $\left(\operatorname{Pr}_{4}\right)$ & A & 5.46 & 0.0274 & 0.1737 \\
\hline
\end{tabular}

Table 4 | Results of significant multiple regressions analyses of nematodes, litter and fine root biomass (g dry weight). Capitals indicate a significant effect of litter or roots of a distinct tree species. B: beech, A: ash, L: lime. PI: Plant feeders, Fu: Fungal feeders, $\mathrm{Pr}$ : predators, Ba: bacterial feeders, subscript numbers indicate the corresponding $c-p$ classification according to Bongers (1990). 

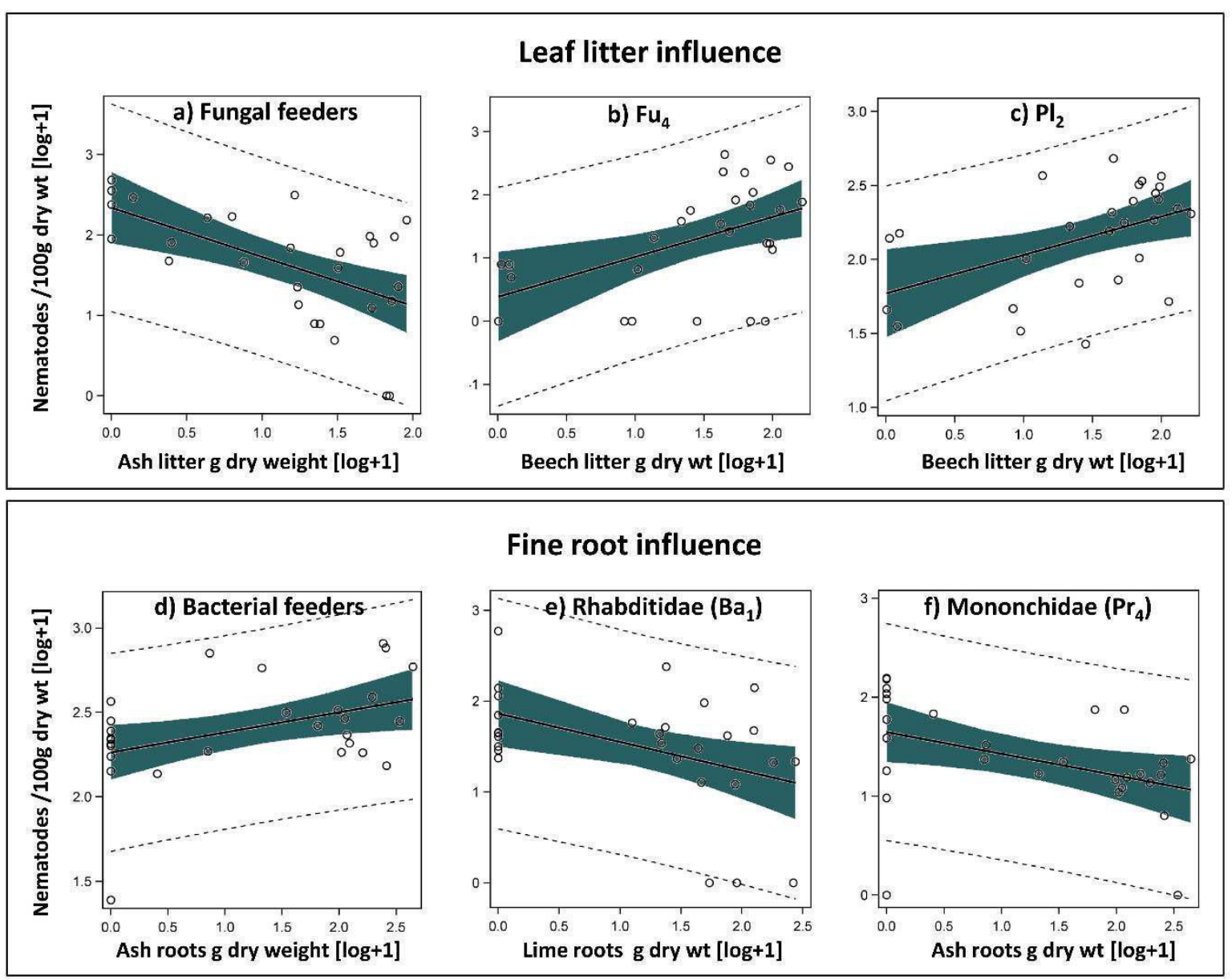

Figure 6 | Significant regressions between soil nematode density and tree species specific litter (left) and fine root (right) biomass with confidence intervals at 95\% (shaded). PI: plant feeders, Ba: bacterial feeders, Fu: fungal feeders, Pr: predators, subscript numbers indicate the corresponding $c-p$ classification according to Bongers (1990).

biomass (Fig. 6). The density of fungal feeders correlated negatively with ash leaf litter biomass, whereas beech litter biomass correlated positively with densities of $\mathrm{Fu}_{4}$ (Leptonchidae), $\mathrm{PI}_{2}$ and the Tylenchidae dominating $\mathrm{PI}_{2}$, whereas fine roots had no effect on these taxa (Table 4). Increased ash fine root biomass was associated with increased density of bacterial-feeding nematodes. By contrast, fine root biomass of lime correlated negatively with the density of bacterial-feeding Rhabditidae $\left(\mathrm{Ba}_{1}\right)$ and ash fine root biomass correlated negatively with the density of predatory Mononchidae (Fig. 6, Table 4). 


\section{Discussion}

\section{Tree diversity}

The present study did reveal impact of tree species identity but not diversity on nematode density and community composition, suggesting tree identity is predominantly modulating the nematode fauna. Similarly, other studies found plant species identity to surpass effects of species diversity on soil biota (De Deyn et al., 2004; Ball et al., 2009; Nadrowski et al., 2010). The results suggest that particular tree species modify the importance of bacterial and fungal energy channels as indicated by bacterial and fungal-feeding nematodes.

\section{Ash}

In mono-specific stands, ash beneficially affected bacterial-feeding nematodes and in parallel detrimentally affected fungal feeders. Bacteria are positively influenced by neutral to high soil $\mathrm{pH}$ what is in line with high Base saturation, soil $\mathrm{pH}$ and exchangeable $\mathrm{Mg}^{2+}$ were high in ash clusters (Langenbruch et al., 2012) and is reported to increase bacteria positively, whereas fungi tolerate a wider range of soil pH (Aciego Pietri and Brookes, 2008; Rousk et al., 2009; Strickland and Rousk, 2010). Indeed, bacterial biomass was highest in ash only clusters as indicated by PLFA analysis (A. Scheibe, unpublished results). Positive correlations of exchangeable $\mathrm{Mg}^{2+}$ and $\mathrm{Ca}^{2+}$ with the amount of ash leaf litter suggests that the beneficial effect of ash is mediated via its litter (Langenbruch et al., 2012). In a common garden experiment with 14 tree species planted in monocultures on a prior pine-dominated forest, Reich et al., (2005) showed ash also to increase soil pH, exchangeable calcium and base saturation arguing for specific species effects of plants on soil conditions forming distinct environments. Moreover, the density of bacterial-feeding nematodes correlated positively 
with ash fine root biomass suggesting that belowground input via roots contributed to fostering bacterial feeders in ash clusters. The amount of dissolved organic carbon (DOC) in forest soils peaks in spring and decreases during litter fall (Weintraub et al., 2007; Kaiser, Koranda, et al., 2010). High amounts of DOC in soil in spring match the period when only small amounts of ash leaf litter were present at our study sites, as it decomposes rapidly, i.e., within six months after litter fall (Jacob et al., 2009, 2010). We therefore suggest seasonal variations in resource inputs to induce changes in the importance of leaf litter- and root-derived resources in soil food webs and therefore likely affect the balance between aboveground to belowground plant carbon input.

The detrimental effect of ash on fungal-feeding nematodes presumably was mediated via ash leaf litter as indicated by negative correlations between the density of fungal-feeding nematodes and the amount of ash leaf litter. Low densities of fungal-feeding nematodes in ash clusters were associated with low fungal-to-bacteria ratio as indicated by PLFA markers (A. Scheibe, unpublished results). However, as indicated by the marker PLFA 18:2w6,9 (Frostegård and Bååth, 1996) fungal biomass was highest in ash only clusters (as also overall microbial biomass) suggesting that the impact of ash was not based on a depletion of fungal resources. Both competition for fungi as food but a lso control of nematodes by predators could have contributed to this pattern. A range of soil invertebrates feed on both fungi and nematodes, including oribatid mites, earthworms and collembolans (Ruess et al., 2005; Curry and Schmidt, 2007; Pollierer et al., 2012). The density of both oribatid mites and collembolans were low in ash as compared to beech clusters, whereas the density of earthworms did not vary between clusters (Eissfeller et al., 2013; V. Eissfeller and S.Cesarz, unpublished results). This suggests that low density of fungal-feeding nematodes was not due to increased 
competition for fungi as food or top-down control by predators in ash clusters. Probably, ash roots are exclusively colonized by AM fungi resulting in only low amounts of EM fungi in pure ash clusters. The high amount of fungal biomass in ash clusters indicated by the PLFA marker 18:2w6,9, indicating EM fungi and saprotrophic fungi but no AM fungi (Ruess and Chamberlain, 2010), therefore refers mainly to saprotrophic fungi. This suggests EM fungi to contribute strongly to the nutrition of nematodes and is supported by a compound-specific ${ }^{13} \mathrm{C}$ fatty acid analysis of Pollierer et al. (2012) for other soil organisms such as the Lepidocyrtus lanuginosus (Collembola) and Trichoniscus pusillus (Isopod). Further, nematodes are known to preferentially feed and to multiply faster on ectomycorrhiza compared to saprotrophic fungi (Mankau and Mankau, 1963; Ruess and Dighton, 1996).

Plant feeding Hoplolaimidae $\left(\mathrm{Pl}_{3}\right)$ benefited from the presence of both ash and beech and reached maximum density in BA and A clusters, whereas densities were low in B clusters. Tree fine root biomass and fine root composition did not differ among mono-specific ( $A$ and $B$ clusters) and diverse tree stands (e.g., BA clusters) at the same site (Meinen et al., 2009a; Jacob et al., 2012) and suggest no direct impact of tree root biomass and composition but other factors to affect plant feeding Hoplolaimidae. Herb species richness and cover was influenced by tree species diversity (Vockenhuber et al., 2011) and may have contributed to the flourishing of Hoplolaimidae in BA and A clusters by increasing host diversity and availability. For instance, spring ephemeral herbs are a higher nutrient sink in spring compared to trees (Mabry et al., 2008; Ellum et al., 2010) whereof Hoplolaimidae may have benefitted.

As for plant feeders, seasonal fluctuations in nutrient supply by herbs and trees may have caused the reduction in predatory Mononchidae. These are known to sensitively respond to 
altered soil nutrient concentrations (Neher and Darby, 2009), and such fluctuations are likely associated with the shift in the input of above- vs. belowground components in ash clusters. Generally, Mononchidae feed on nematodes (Loof, 1999) but apparently did not profit from higher densities of bacterial-feeding nematodes which benefited from ash. Presumably, Mononchidae, as typical $K$-strategists, more sensitively respond to variations in environmental conditions rather than food (Bongers 1990; Ferris et al. 2001). Although bacteria and bacterialfeeders were fostered by ash leaf litter, no "long" food webs with substantial biomass at higher trophic levels could be sustained as indicat ed by Mononchidae. Thus, the litter supply did not result in an enhanced carbon and energy transfer to higher trophic levels, i.e., increased propagation of carbon to upper trophic le vels potentially due to the lack of EM fungi and EM fungi consumers.

\section{Beech}

In contrast to ash, beech beneficially affected fungal feeders and this was most pronounced in $\mathrm{Fu}_{4}$ nematodes. These changes in trophic structure were associated with low soil $\mathrm{pH}$, low concentrations of exchangeable $\mathrm{Mg}^{2+}$, and low carbon-to-nitrogen and lignin-to-nitrogen ratios in beech only clusters (Langenbruch et al. 2012), i.e., factors known to favor fungi over bacteria (Aciego Pietri and Brookes, 2008; Rousk et al., 2009; Strickland and Rousk, 2010). As the density of $\mathrm{Fu}_{4}$ nematodes correlated more closely with the amount of beech leaf litter than with beech fine root biomass the favorable effect of beech presumably was mediated by beech leaf litter associated factors such as increased fungal-to-bacterial ratio likely increasing the competitive strength of fungi. 
Surprisingly, the plant-feeding Tylenchidae, the do minant group in $\mathrm{Pl}_{2}$ nematodes, correlated positively with the amount of beech leaf litter and not with root biomass. This suggests that rather than feeding on living roots these nematodes rely on fungi associated with beech leaf litter, and illustrates the ambiguity in ascribing nematodes bearing a stylet to root or fungal feeders. Supporting this conclusion species of the tylenchid genera Filenchus and Tylenchus have been shown to feed on fungi (Wood, 1973; Okada et al., 2002, 2005; Okada and Kadota, 2003). Generally, there is a lack of evidence that Tylenchidae pierce plant cells and live as root feeders (Yeates, 1999).

\section{Lime}

Generally, increasing proportion of lime in clusters decreased total nematode density (Pearsons Rho $=-0.39904, P=0.0354$ ), in particular bacterial-feeding Rhabditidae were negatively affected, whereas Tylenchidae benefitted from lime presence. The detrimental effect of lime could not be attributed to any of the soil factors assessed but the decomposition constant k of lime litter correlated positively with the density of earthworms (Jacob et al., 2009) suggesting that nematodes suffer from the decline of leaf litter in the litter layer and direct interactions with earthworms (Yeates, 1981). The detrimental effect of lime also applied to omnivorous nematodes, which were neither affected by leaf litter nor by roots. Although ash and lime leaf litter both are regarded as high quality food for decomposers these tree species negatively affected soil nematodes suggesting that other factors than litter quality per se, e.g., rhizodeposits or biotic interactions also structure soil nematode communities. 


\section{Conclusions}

The results suggest that tree species identity is more important than tree species diversity in structuring nematode communities and associated soil processes. Ash increased the density of bacterial-feeding nematodes and reduced the number of fungal feeders, indicating distinct changes in regulatory forces of soil food webs. Beech detrimentally affected bacterial feeders but favored fungal feeders probably via $\mathrm{pH}$-mediated increase in the fungal-to-bacterial ratio.The effect of lime was less pronounced but te nded to be generally negative. The results indicate that both leaf litter and roots influence the nematode community, with the effects being driven not solely by resource quality but additionally by availability, i.e. seasonal shifts in dominant belowground resources. In sum the structure of soil food webs varies markedly with tree species pointing to the importance of variations in plant resources, i.e., leaf litter quality and root exudates, as strong bottom-up regulating factors of microbial communities and energy channels of decomposer systems.

\section{Acknowledgements}

Simone Cesarz gratefully acknowledges funding by the Ministry of Science and Culture of Lower Saxony and the 'Niedersächsisches Vorab' as part of the Cluster of Excellence 'Functional Biodiversity Research'. The study was associated to Graduate School 1086 which was funded by the Deutsche Forschungsgemeinschaft (DFG). We thank Andrea Scheibe for providing data on PLFA analysis and Michael Ackermann and René Seiml-Buchinger for 
providing help in nematode identification. We thank two anonymous reviewer for their comments which considerably improved our work.

\section{References}

Aciego Pietri, J.C., Brookes, P.C., 2008. Relationships between soil pH and microbial properties in a UK arable soil. Soil Biology and Biochemistry 40, 1856-1861.

Albers, D., Schaefer, M., Scheu, S., 2006. Incorporation of plant carbon into the soil animal food web of an arable system. Ecology 87, 235-245.

Ball, B.A., Bradford, M.A., Coleman, D.C., Hunter, M.D., 2009. Linkages between below and aboveground communities: Decomposer responses to simulated tree species loss are largely additive. Soil Biology and Biochemistry 41, 1155-1163.

Bird, J.A., Herman, D.J., Firestone, M.K., 2011. Rhizosphere priming of soil organic matter by bacterial groups in a grassland soil. Soil Biology and Biochemistry 43, 718-725.

Bongers, T., 1990. The maturity index: an ecological measure of environmental disturbance based on nematode species composition. Oecologia 83, 14-19.

Cadish, G., Giller, K.E., 1997. Driven by nature: plant litter and decomposition. CABI, Wallingford, UK.

Cesarz, S., Fender, A.-C., Beyer, F., Valtanen, K., Pfeiffer, B., Gansert, D., Hertel, D., Polle, A., Daniel, R., Leuschner, C., Scheu, S., 2013. Roots from beech (Fagus sylvatica L.) and ash (Fraxinus excelsior L.) differentially affect soil microorganisms and carbon dynamics. Soil Biology and Biochemistry doi: 10.1016/j.soilbi o.2013.02.003.

Coûteaux, M.-M., Bottner, P., Berg, B., 1995. Litter decomposition, climate and litter quality. Trends in Ecology \& Evolution 10, 63-66.

Curry, J.P., Schmidt, O., 2007. The feeding ecology of earthworms - A review. Pedobiologia 50, 463-477.

De Deyn, G.B., Raaijmakers, C.E., Ruijven, J. Van, Berendse, F., van der Putten, W.H., 2004. Plant species identity and diversity effects on different trophic levels of nematodes in the soil food web. Oikos 106, 576-586.

Der, G., Everitt, B.S., 2002. A handbook of statistical analyses using SAS. Chapman and Hall/CRC, London. 
Eisenhauer, N., Cesarz, S., Koller, R., Worm, K., Reich, P.B., 2012. Global change belowground: impacts of elevated $\mathrm{CO}_{2}$, nitrogen, and summer drought on soil food webs and biodiversity. Global Change Biology 18, 435-447.

Ellum, D.S., Ashton, M.S., Siccama, T.G., 2010. Spatial pattern in herb diversity and abundance of second growth mixed deciduous-evergreen forest of southern New England, USA. Forest Ecology and Management 259, 1416-1426.

Endlweber, K., Ruess, L., Scheu, S., 2009. Collembola switch diet in presence of plant roots thereby functioning as herbivores. Soil Biology and Biochemistry 41, 1151-1154.

Fender, A.-C., Gansert, D., Jungkunst, H.F., Fiedler, S., Beyer, F., Schützenmeister, K., Thiele, B., Valtanen, K., Polle, A., Leuschner, C., 2013. Root-induced tree species effects on the source/sink strength for greenhouse gases $\left(\mathrm{CH}_{4}, \mathrm{~N}_{2} \mathrm{O}\right.$ and $\left.\mathrm{CO}_{2}\right)$ of a temperate deciduous forest soil. Soil Biology and Biochemistry 57, 587-597.

Ferris, H., Bongers, T., de Goede, R.G.M., 2001. A framework for soil food web diagnostics: extension of the nematode faunal analysis concept. Applied Soil Ecology 18, 13-29.

Frostegård, A., Bååth, E., 1996. The use of phospholipid fatty acid analysis to estimate bacterial and fungal biomass in soil. Biology and Fertility of Soils 22, 59-65.

Grayston, S.J., Vaughan, D., Jones, D., 1996. Rhizosphere carbon flow in trees, in comparison with annual plants: the importance of root exudation and its impact on microbial activity and nutrient availability. Applied Soil Ec ology 5, 29-56.

Guckland, A., Jacob, M., Flessa, H., Thomas, F.M., Leuschner, C., 2009. Acidity, nutrient stocks, and organic-matter content in soils of a temperate deciduous forest with different abundance of European beech (Fagus sylvatica L.). Journal of Plant Nutrition and Soil Science 172, 500-511.

Hölscher, D., Hertel, D., Leuschner, C., Hottkowitz, M., 2002. Tree species diversity and soil patchiness in a temperate broad-leaved forest with limited rooting space. Flora 197, 118-125.

Jacob, A., Hertel, D., Leuschner, C., 2012. On the significance of belowground overyielding in temperate mixed forests: separating species identity and species diversity effects. Oikos doi: 10.1111/j.1600-0706.2012.20476.x.

Jacob, M., Viedenz, K., Polle, A., Thomas, F.M., 2010. Leaf litter decomposition in temperate deciduous forest stands with a decreasing fraction of beech (Fagus sylvatica). Oecologia 164, 1083-1094.

Jacob, M., Weland, N., Platner, C., Schaefer, M., Leuschner, C., Thomas, F.M., 2009. Nutrient release from decomposing leaf litter of temperate deciduous forest trees along a gradient of increasing tree species diversity. Soil Biology and Biochemistry 41, 21222130. 
Jones, D.L., Hodge, A., Kuzyakov, Y., 2004. Plant and mycorrhizal regulation of rhizodeposition. New Phytologist 163, 459-480.

Jones, D.L., Nguyen, C., Finlay, R.D., 2009. Carbon flow in the rhizosphere: carbon trading at the soil-root interface. Plant and Soil 321, 5-33.

Kaiser, C., Koranda, M., Kitzler, B., Fuchslueger, L., Schnecker, J., Schweiger, P., Rasche, F., Zechmeister-Boltenstern, S., Sessitsch, A., Richter, A., 2010. Belowground carbon allocation by trees drives seasonal patterns of extracellular enzyme activities by altering microbial community composition in a beech forest soil. New Phytologist 187 , 843-858.

Keith, A.M., Brooker, R.W., Osler, G.H.R., Chapman, S.J., Burslem, D.F.R.P., van der Wal, R., 2009. Strong impacts of belowground tree inputs on soil nematode trophic composition. Soil Biology and Biochemistry 41, 1060-1065.

Kuzyakov, Y., 2010. Priming effects: Interactions between living and dead organic matter. Soil Biology and Biochemistry 42, 1363-1371.

Lang, C., Seven, J., Polle, A., 2011. Host preferences and differential contributions of deciduous tree species shape mycorrhizal species richness in a mixed Central European forest. Mycorrhiza 21, 297-308.

Langenbruch, C., Helfrich, M., Flessa, H., 2012. Effects of beech (Fagus sy/vatica), ash (Fraxinus excelsior) and lime (Tilia spec.) on soil chemical properties in a mixed deciduous forest. Plant and Soil 352, 389-403.

Loof, P.A.A., 1999. Nematoda, Adenophorea (Dorylaimida). Spektrum Akademischer Verlag, Heidelberg.

Mabry, C.M., Gerken, M.E., Thompson, J.R., 2008. Seasonal storage of nutrients by perennial herbaceous species in undisturbed and disturbed deciduous hardwood forests. Applied Vegetation Science 11, 37-44.

Mankau, R., Mankau, S.K., 1963. The role of mycophagous nematodes in the soil. I. The relationships of Aphelenchus avenae to phytopathogenic soil fungi, in: Docksen, J., van der Drift, J. (Eds.), Phytopathology. North-Holland Publishing Company, Amsterdam, pp. 271-280.

McKinley, D.C., Ryan, M.G., Birdsey, R.A., Giardina, C.P., Harmon, M.E., Heath, L.S., Houghton, R.A., Jackson, R.B., Morrison, J.F., Murray, B.C., Pataki, D.E., Skog, K.E., 2011. A synthesis of current knowledge on forests and carbon storage in the United States. Ecological Applications 21, 1902-1924.

Meinen, C., Hertel, D., Leuschner, C., 2009a. Biomass and morphology of fine roots in temperate broad-leaved forests differing in tree species diversity: is there evidence of below-ground overyielding? Oecologia 161, 99-111. 
Meinen, C., Leuschner, C., Ryan, N.T., Hertel, D., 2009b. No evidence of spatial root system segregation and elevated fine root biomass in multi-species temperate broad-leaved forests. Trees 23, 941-950.

Morin, X., Fahse, L., Scherer-Lorenzen, M., Bugmann, H., 2011. Tree species richness promotes productivity in temperate forests through strong complementarity between species. Ecology Letters 14, 1211-1219.

Nadrowski, K., Wirth, C., Scherer-Lorenzen, M., 2010. Is forest diversity driving ecosystem function and service? Current Opinion in Environmen tal Sustainability 2, 75-79.

Neher, D.A., Darby, B.J., 2009. Community indices, in: Wilson, M.J., Kakouli-Duarte, T. (Eds.), Nematodes as Environmental Indicators. CABI, Wallingford, UK, pp. 107-123.

Okada, H., Harada, H., Kadota, I., 2005. Fungal-feeding habits of six nematode isolates in the genus Filenchus. Soil Biology and Biochemistry 37, 1113-1120.

Okada, H., Kadota, I., 2003. Host status of 10 fungal isolates for two nematode species, Filenchus misellus and Aphelenchus avenae. Soil Biology and Biochemistry 35, 16011607.

Okada, H., Tsukiboshi, T., Kadota, I., 2002. Mycetophagy in Filenchus misellus (Andrassy, 1958) Lownsbery \& Lownsbery, 1985 (Nematoda: Tylenchidae), with notes on its morphology. Nematology 4, 795-801.

Pollierer, M.M., Dyckmans, J., Scheu, S., Haubert, D., 2012. Carbon flux through fungi and bacteria into the forest soil animal food web as in dicated by compound specific ${ }^{13} \mathrm{C}$ fatty acid analysis. Functional Ecology 26, 978-990.

Pollierer, M.M., Langel, R., Körner, C., Maraun, M., Scheu, S., 2007. The underestimated importance of belowground carbon input for forest soil animal food webs. Ecology Letters 10, 729-36.

Reich, P.B., Oleksyn, J., Modrzynski, J., Mrozinski, P., Hobbie, S.E., Eissenstat, D.M., Chorover, J., Chadwick, O.A., Hale, C.M., Tjoelker, M.G., 2005. Linking litter calcium, earthworms and soil properties: a common garden test with 14 tree species. Ecology Letters 8, 811818.

Rousk, J., Brookes, P.C., Bååth, E., 2009. Contrasting soil pH effects on fungal and bacterial growth suggest functional redundancy in carbon mineralization. Applied and Environmental Microbiology 75, 1589-96.

Ruess, L., 1995. Studies on the nematode fauna of a $n$ acid forest soil: spatial disturbance and extraction. Nematologica 41, 229-239.

Ruess, L., Chamberlain, P.M., 2010. The fat that matters: Soil food web analysis using fatty acids and their carbon stable isotope signature. Soil Biology and Biochemistry 42, 1898-1910. 
Ruess, L., Dighton, J., 1996. Cultural studies on soil nematodes and their fungal hosts. Nematologica 42, 330-346.

Ruess, L., Ferris, H., 2004. Decomposition pathways and successional changes. Nematology Monographs \& Perspectives 2, 547-556.

Ruess, L., Tiunov, A., Haubert, D., Richnow, H.H., Häggblom, M.M., Scheu, S., 2005. Carbon stable isotope fractionation and trophic transfer of fatty acids in fungal based soil food chains. Soil Biology and Biochemistry 37, 945-953.

Scandellari, F., Ventura, M., Gioacchini, P., Vittori Antisari, L., Tagliavini, M., 2010. Seasonal pattern of net nitrogen rhizodeposition from peach (Prunus persica (L.) Batsch) trees in soils with different textures. Agriculture, Ecosystems \& Environment 136, 162-168.

Scherber, C., Eisenhauer, N., Weisser, W.W., Schmid, B., Voigt, W., Fischer, M., Schulze, E.-D., Roscher, C., Weigelt, A., Allan, E., Bessler, H., Bonkowski, M., Buchmann, N., Buscot, F., Clement, L.W., Ebeling, A., Engels, C., Halle, S., Kertscher, I., Klein, A.-M., Koller, R., König, S., Kowalski, E., Kummer, V., Kuu, A., Lange, M., Lauterbach, D., Middelhoff, C., Migunova, V.D., Milcu, A., Müller, R., Partsch, S., Petermann, J.S., Renker, C., Rottstock, T., Sabais, A., Scheu, S., Schumacher, J., Temperton, V.M., Tscharntke, T., 2010. Bottom-up effects of plant diversity on multitrophic interactions in a biodiversity experiment. Nature 468, 553-556.

Strickland, M.S., Rousk, J., 2010. Considering fung al:bacterial dominance in soils - Methods, controls, and ecosystem implications. Soil Biology and Biochemistry 42, 1385-1395.

Swift, M.J., Heal, O.W., Anderson, J.M., 1979. Decomposition in terrestrial ecosystems. University of California Press, Berkeley.

Ter Braak, C.J.F., Smilauer, P., 2002. CANOCO Reference Manual and CanoDraw for Windows User's Guide: Software for Canonical Community Ordination (version 4.5). Ithaca, NY, USA.

Vockenhuber, E.A., Scherber, C., Langenbruch, C., Meißner, M., Seidel, D., Tscharntke, T., 2011. Tree diversity and environmental context predict herb species richness and cover in Germany's largest connected deciduous forest. Perspectives in Plant Ecology, Evolution and Systematics 13, 111-119.

Wardle, D.A., 2002. Communities and Ecosystems: Linking the Aboveground and Belowground Components. Princeton University Press, Princeton and Oxford.

Wardle, D.A., Bardgett, R.D., Klironomos, J.N., Setälä, H., van der Putten, W.H., Wall, D.H., 2004. Ecological linkages between aboveground and belowground biota. Science 304, 1629-1633.

Wardle, D.A., Verhoef, H.A., Clarholm, M., 1998. Trophic relationships in the soil microfoodweb: predicting the responses to a changing global. Global Change Biology 4, 713-727. 
Weintraub, M.N., Scott-Denton, L.E., Schmidt, S.K., Monson, R.K., 2007. The effects of tree rhizodeposition on soil exoenzyme activity, dissolved organic carbon, and nutrient availability in a subalpine forest ecosystem. Oecologia 154, 327-338.

Wood, F.H., 1973. Nematode feeding relationships. Soil Biology and Biochemistry 5, 593-601.

Yeates, G.W., 1981. Soil nematodes depressed by earthworms. Pedobiologia 22, 191-195.

Yeates, G.W., 1999. Effects of plants on nematode community structure. Annual Review of Phytopathology 37, 127-149.

Yeates, G.W., Bongers, T., de Goede, R.G.M., Freckman, D.W., Georgieva, S.S., 1993. Feeding habits in soil nematode families and genera - an outline for soil ecologist. Journal of Nematology 25, 315-331. 
Supplementary 
Table S1 | Means \pm SE of abundance (Individuals/g soil dry wei ght) of families of soil nematodes as affected by beech (B), ash (A) and lime (L) presence (+) or absence (-), respectively. The respective cluster combinations are given in parenthesis. PI: plant feeders, Ba: bacterial feeders, Fu: fungal feeders, Om: omnivores, Pr: predators, subscript numbers indicate the corresponding $c-p$ classification according to Bongers (1990).

\begin{tabular}{|c|c|c|c|c|c|c|c|c|c|c|c|c|c|c|c|c|c|c|c|c|c|c|}
\hline & & \multicolumn{9}{|c|}{$-B$} & \multicolumn{12}{|c|}{$+\mathrm{B}$} \\
\hline & & \multirow{2}{*}{\multicolumn{3}{|c|}{$\begin{array}{l}-A \\
+L\end{array}$}} & \multicolumn{6}{|c|}{$+\mathrm{A}$} & \multicolumn{6}{|c|}{$-A$} & \multicolumn{6}{|c|}{$+\mathrm{A}$} \\
\hline & & & & & \multirow{2}{*}{\multicolumn{3}{|c|}{$\frac{-L}{\text { (Ash) }}$}} & \multirow{2}{*}{\multicolumn{3}{|c|}{$\frac{+\mathrm{L}}{\text { sh-Lime) }}$}} & \multirow{2}{*}{\multicolumn{3}{|c|}{$\frac{-L}{\text { 3eech) }}$}} & \multicolumn{3}{|c|}{$+\mathrm{L}$} & \multirow{2}{*}{\multicolumn{3}{|c|}{$-\mathrm{L}$}} & \multicolumn{3}{|c|}{$+\mathrm{L}$} \\
\hline & & & ime & & & & & & & & & & & (Beec & $\mathrm{h}-\mathrm{Li}$ & ime) & & & & (Beech & -Ash & ר-Lime) \\
\hline & & Mean & & SE & Mean & & SE & Mean & & SE & Mean & & SE & Mean & & SE & Mean & & SE & Mean & & SE \\
\hline \multicolumn{2}{|c|}{ Families } & & & & & & & & & & & & & & & & & & & & & \\
\hline $\mathrm{Pl}_{2}$ & Tylenchidae & 109.96 & \pm & 22.09 & 106.54 & \pm & 51.76 & 169.29 & \pm & 41.55 & 265.88 & \pm & 86.98 & 198.85 & \pm & 43.77 & 78.48 & \pm & 41.35 & 293.39 & \pm & 50.28 \\
\hline $\mathrm{Pl}_{2}$ & Tylodoridae & 16.21 & \pm & 16.21 & 0.00 & \pm & 0.00 & 3.11 & \pm & 1.83 & 3.66 & \pm & 3.66 & 6.77 & \pm & 6.77 & 0.00 & \pm & 0.00 & 31.48 & \pm & 23.85 \\
\hline $\mathrm{Pl}_{3}$ & Criconematidae* & 9.58 & \pm & 2.10 & 0.00 & \pm & 0.00 & 1.68 & \pm & 1.68 & 7.96 & \pm & 4.62 & 0.00 & \pm & 0.00 & 21.98 & \pm & 12.47 & 20.34 & \pm & 9.14 \\
\hline $\mathrm{Pl}_{3}$ & Dolichoridae* & 8.32 & \pm & 3.82 & 3.97 & \pm & 3.97 & 1.34 & \pm & 1.34 & 3.66 & \pm & 3.66 & 0.00 & \pm & 0.00 & 0.00 & \pm & 0.00 & 13.64 & \pm & 8.96 \\
\hline $\mathrm{Pl}_{3}$ & Hoplolaimidae & 28.89 & \pm & 19.20 & 142.34 & \pm & 72.31 & 45.00 & \pm & 13.31 & 7.56 & \pm & 4.37 & 0.00 & \pm & 0.00 & 265.14 & \pm & 224.20 & 94.15 & \pm & 81.41 \\
\hline $\mathrm{Pl}_{3}$ & Paratylenchidae* & 2.71 & \pm & 1.68 & 0.00 & \pm & 0.00 & 3.45 & \pm & 2.09 & 7.56 & \pm & 4.37 & 8.69 & \pm & 6.39 & 0.00 & \pm & 0.00 & 9.84 & \pm & 3.57 \\
\hline $\mathrm{Pl}_{3}$ & Pratylenchidae* & 0.00 & \pm & 0.00 & 0.00 & \pm & 0.00 & 0.00 & \pm & 0.00 & 0.00 & \pm & 0.00 & 20.31 & \pm & 20.31 & 0.00 & \pm & 0.00 & 0.00 & \pm & 0.00 \\
\hline $\mathrm{Ba}_{1}$ & Bunonematidae* & 0.00 & \pm & 0.00 & 0.00 & \pm & 0.00 & 0.00 & \pm & 0.00 & 0.00 & \pm & 0.00 & 0.00 & \pm & 0.00 & 3.43 & \pm & 3.43 & 0.00 & \pm & 0.00 \\
\hline $\mathrm{Ba}_{1}$ & Monhysteridae & 26.37 & \pm & 19.05 & 46.73 & \pm & 15.71 & 30.39 & \pm & 9.56 & 39.26 & \pm & 14.07 & 35.00 & \pm & 21.62 & 19.10 & \pm & 14.89 & 47.03 & \pm & 12.42 \\
\hline $\mathrm{Ba}_{1}$ & Rhabditidae & 32.80 & \pm & 12.53 & 104.89 & \pm & 52.96 & 38.37 & \pm & 7.66 & 30.01 & \pm & 10.71 & 44.36 & \pm & 17.95 & 27.75 & \pm & 11.14 & 216.09 & \pm & 126.91 \\
\hline $\mathrm{Ba}_{2}$ & Cephalobidae & 26.77 & \pm & 11.91 & 142.97 & \pm & 86.22 & 55.16 & \pm & 10.26 & 37.87 & \pm & 23.95 & 44.75 & \pm & 7.74 & 80.18 & \pm & 41.09 & 98.86 & \pm & 17.59 \\
\hline $\mathrm{Ba}_{2}$ & Plectidae & 49.71 & \pm & 37.21 & 77.94 & \pm & 28.46 & 22.19 & \pm & 4.34 & 36.29 & \pm & 15.99 & 35.49 & \pm & 13.76 & 115.77 & \pm & 71.79 & 49.73 & \pm & 23.75 \\
\hline $\mathrm{Ba}_{3}$ & Bastianidae & 7.26 & \pm & 3.20 & 17.61 & \pm & 13.23 & 3.54 & \pm & 3.54 & 8.14 & \pm & 4.71 & 32.48 & \pm & 8.81 & 55.28 & \pm & 34.24 & 20.66 & \pm & 10.89 \\
\hline $\mathrm{Ba}_{3}$ & Prismatolaimdae* & 3.45 & \pm & 3.45 & 18.60 & \pm & 16.27 & 7.82 & \pm & 3.31 & 0.00 & \pm & 0.00 & 4.78 & \pm & 3.20 & 10.84 & \pm & 6.27 & 11.89 & \pm & 6.89 \\
\hline $\mathrm{Ba}_{3}$ & Teratocephalidae & 7.57 & \pm & 4.54 & 10.55 & \pm & 7.94 & 2.74 & \pm & 1.66 & 19.00 & \pm & 11.51 & 7.15 & \pm & 5.46 & 33.99 & \pm & 16.49 & 11.68 & \pm & 7.25 \\
\hline $\mathrm{Ba}_{4}$ & Alaimidae & 22.16 & \pm & 16.02 & 27.53 & \pm & 6.66 & 41.33 & \pm & 17.79 & 40.19 & \pm & 17.59 & 41.51 & \pm & 13.43 & 37.38 & \pm & 6.49 & 40.98 & \pm & 19.50 \\
\hline $\mathrm{Fu}_{2}$ & Anguinidae* & 1.42 & \pm & 1.42 & 0.00 & \pm & 0.00 & 1.68 & \pm & 1.68 & 0.00 & \pm & 0.00 & 10.16 & \pm & 10.16 & 0.00 & \pm & 0.00 & 19.60 & \pm & 19.60 \\
\hline $\mathrm{Fu}_{2}$ & Aphelenchoididae* & 2.83 & \pm & 2.83 & 15.00 & \pm & 5.21 & 10.09 & \pm & 10.09 & 18.48 & \pm & 9.89 & 0.00 & \pm & 0.00 & 7.74 & \pm & 6.00 & 15.43 & \pm & 11.99 \\
\hline $\mathrm{Fu}_{2}$ & Aphelenchidae* & 2.32 & \pm & 2.32 & 5.60 & \pm & 5.60 & 2.40 & \pm & 1.40 & 0.00 & \pm & 0.00 & 15.35 & \pm & 6.20 & 0.00 & \pm & 0.00 & 18.38 & \pm & 10.69 \\
\hline $\mathrm{Fu}_{3}$ & Diphterophoridae* & 1.42 & \pm & 1.42 & 24.28 & \pm & 15.29 & 9.01 & \pm & 3.26 & 0.00 & \pm & 0.00 & 1.39 & \pm & 1.39 & 14.06 & \pm & 12.24 & 4.21 & \pm & 4.21 \\
\hline $\mathrm{Fu}_{4}$ & Leptonchidae & 26.12 & \pm & 12.62 & 3.97 & \pm & 3.97 & 18.77 & \pm & 7.21 & 280.41 & \pm & 81.47 & 106.44 & \pm & 46.72 & 20.67 & \pm & 18.46 & 80.95 & \pm & 49.19 \\
\hline $\mathrm{Pr}_{3}$ & Tripylidae & 5.61 & \pm & 4.40 & 33.92 & \pm & 31.12 & 11.12 & \pm & 3.78 & 13.87 & \pm & 13.87 & 8.62 & \pm & 3.58 & 41.05 & \pm & 21.38 & 11.27 & \pm & 6.59 \\
\hline $\mathrm{Pr}_{4}$ & Anatonchidae* & 0.00 & \pm & 0.00 & 0.00 & \pm & 0.00 & 1.34 & \pm & 1.34 & 0.00 & \pm & 0.00 & 5.76 & \pm & 5.76 & 0.00 & \pm & 0.00 & 0.00 & \pm & 0.00 \\
\hline $\mathrm{Pr}_{4}$ & Mononchidae & 33.28 & \pm & 13.68 & 26.53 & \pm & 13.63 & 36.72 & \pm & 23.86 & 76.10 & \pm & 28.90 & 58.05 & \pm & 29.76 & 55.01 & \pm & 32.43 & 14.72 & \pm & 8.07 \\
\hline $\mathrm{Om}_{4}$ & Aporcelaimidae & 49.65 & \pm & 24.14 & 45.03 & \pm & 6.91 & 34.82 & \pm & 11.12 & 38.35 & \pm & 18.80 & 89.03 & \pm & 52.42 & 64.69 & \pm & 34.64 & 71.14 & \pm & 26.87 \\
\hline $\mathrm{Om}_{5}$ & Qudsianematidae & 40.62 & \pm & 17.41 & 12.89 & \pm & 2.28 & 9.81 & \pm & 6.32 & 43.43 & \pm & 23.47 & 6.70 & \pm & 2.80 & 42.08 & \pm & 21.69 & 29.61 & \pm & 7.99 \\
\hline $\mathrm{Om}_{5}$ & Thornenematidae & 15.11 & \pm & 7.62 & 41.14 & \pm & 25.28 & 9.04 & \pm & 4.62 & 119.50 & \pm & 79.31 & 3.39 & \pm & 3.39 & 51.68 & \pm & 4.64 & 39.21 & \pm & 15.00 \\
\hline
\end{tabular}


Table S2 | ANOVA table of $F$ - and $P$-values on the effects of beech, ash and lime presence and absence, respectively, and all possible interactions on the abundance families of soil nematodes. Pl: plant feeders, Ba: bacterial feeders, Fu: fungal feeders, Om: omnivores, Pr: predators, subscript numbers indicate the corresponding $c-p$ classification according to Bongers (1990). Rare groups are not displayed and were not analyzed with ANOVA due to very low densities $(<2 \%)$.

\begin{tabular}{|c|c|c|c|c|c|c|c|c|c|c|c|c|c|c|c|c|c|c|}
\hline & & \multicolumn{2}{|c|}{ Beech } & & \multicolumn{2}{|c|}{ Ash } & & \multicolumn{2}{|c|}{ Lime } & & \multicolumn{2}{|c|}{$B \times A$} & \multicolumn{2}{|c|}{$B \times L$} & \multicolumn{2}{|c|}{$A \times L$} & \multicolumn{2}{|c|}{$B \times A \times L$} \\
\hline & & $\boldsymbol{F}$ & $P$ & & $\boldsymbol{F}$ & $P$ & & $\boldsymbol{F}$ & $P$ & & $\boldsymbol{F}$ & $P$ & $\boldsymbol{F}$ & $P$ & $\boldsymbol{F}$ & $P$ & $\boldsymbol{F}$ & $P$ \\
\hline \multicolumn{19}{|c|}{ Families } \\
\hline $\mathrm{Pl}_{2}$ & Tylenchidae & 2.37 & 0.1390 & & 1.44 & 0.2433 & & 4.30 & 0.0505 & & \multicolumn{2}{|c|}{ excluded } & 1.42 & 0.2470 & 7.78 & 0.0110 & 0.00 & 1.0000 \\
\hline $\mathrm{Pl}_{2}$ & Tylodoridae & 0.12 & 0.7365 & & 0.04 & 0.8409 & & 2.91 & 0.1028 & & \multicolumn{2}{|c|}{ excluded } & 0.29 & 0.5976 & 1.03 & 0.3225 & 0.50 & 0.4888 \\
\hline $\mathrm{Pl}_{3}$ & Hoplolaimidae & 4.96 & 0.0370 & $\downarrow$ & 13.10 & 0.0016 & $\uparrow$ & 3.46 & 0.0768 & & 0.00 & 1.0000 & \multicolumn{2}{|c|}{ excluded } & 0.00 & 1.0000 & 1.56 & 0.2258 \\
\hline $\mathrm{Ba}_{1}$ & Monhysteridae & 0.48 & 0.4970 & & 0.19 & 0.6696 & & 0.07 & 0.7975 & & \multicolumn{2}{|c|}{ excluded } & 1.40 & 0.2495 & 2.85 & 0.1062 & 2.86 & 0.1058 \\
\hline $\mathrm{Ba}_{1}$ & Rhabditidae & 0.01 & 0.9421 & & 0.58 & 0.4540 & & 2.04 & 0.1681 & & \multicolumn{2}{|c|}{ excluded } & 1.68 & 0.2095 & 0.57 & 0.4589 & 0.27 & 0.6069 \\
\hline $\mathrm{Ba}_{2}$ & Cephalobidae & 0.52 & 0.4780 & & 2.92 & 0.1020 & & 0.52 & 0.4798 & & 0.53 & 0.4736 & \multicolumn{2}{|c|}{ excluded } & 0.53 & 0.4749 & 0.83 & 0.3714 \\
\hline $\mathrm{Ba}_{2}$ & Plectidae & 0.15 & 0.6997 & & 1.66 & 0.2119 & & 1.75 & 0.1996 & & \multicolumn{2}{|c|}{ excluded } & 0.64 & 0.4330 & 0.19 & 0.6665 & 0.29 & 0.5983 \\
\hline $\mathrm{Ba}_{3}$ & Bastianidae & 3.60 & 0.0716 & & 0.16 & 0.6933 & & 0.00 & 0.9836 & & 0.21 & 0.6536 & 1.00 & 0.3288 & \multicolumn{2}{|c|}{ excluded } & 2.97 & 0.0994 \\
\hline $\mathrm{Ba}_{3}$ & Teratocephalidae & 0.63 & 0.4379 & & 0.02 & 0.8839 & & 1.01 & 0.3255 & & \multicolumn{2}{|c|}{ excluded } & 0.04 & 0.8478 & 0.10 & 0.7494 & 0.89 & 0.3568 \\
\hline $\mathrm{Ba}_{4}$ & Alaimidae & 1.23 & 0.2795 & & 0.56 & 0.4642 & & 1.00 & 0.3280 & & 3.81 & 0.0644 & 0.00 & 1.0000 & \multicolumn{2}{|c|}{ excluded } & 0.00 & 1.0000 \\
\hline $\mathrm{Fu}_{4}$ & Leptonchidae & 9.62 & 0.0054 & $\uparrow$ & 12.40 & 0.0021 & $\downarrow$ & 1.66 & 0.2118 & & 0.00 & 1.0000 & \multicolumn{2}{|c|}{ excluded } & 10.00 & 0.0047 & 0.00 & 1.0000 \\
\hline $\operatorname{Pr}_{3}$ & Tripylidae & 0.19 & 0.6666 & & 1.83 & 0.1906 & & 0.37 & 0.5503 & & 0.30 & 0.5915 & \multicolumn{2}{|c|}{ excluded } & 0.64 & 0.4339 & 1.79 & 0.1953 \\
\hline $\mathrm{Pr}_{4}$ & Mononchidae & 0.02 & 0.8801 & & 1.19 & 0.2877 & & 1.83 & 0.1906 & & \multicolumn{2}{|c|}{ excluded } & 1.97 & 0.1750 & 0.63 & 0.4374 & 0.00 & 1.0000 \\
\hline $\mathrm{Om}_{4}$ & Aporcelaimidae & 0.04 & 0.8424 & & 0.54 & 0.4721 & & 0.42 & 0.5237 & & \multicolumn{2}{|c|}{ excluded } & 1.35 & 0.2583 & 1.53 & 0.2290 & 0.00 & 1.0000 \\
\hline $\mathrm{Om}_{5}$ & Qudsianematidae & 0.14 & 0.7163 & & 0.01 & 0.9413 & & 0.60 & 0.4472 & & 6.25 & 0.0207 & 0.41 & 0.5297 & \multicolumn{2}{|c|}{ excluded } & 2.05 & 0.1666 \\
\hline $\mathrm{Om}_{5}$ & Thornenematidae & 2.22 & 0.1514 & & 1.28 & 0.2712 & & 9.70 & 0.0052 & $\downarrow$ & \multicolumn{2}{|c|}{ excluded } & 0.78 & 0.3870 & 2.52 & 0.1275 & 6.11 & 0.0220 \\
\hline
\end{tabular}




\section{Chapter 3}

Roots from beech (Fagus sylvatica L.) and ash (Fraxinus excelsior L.) differentially affect soil microorganisms and carbon dynamics

Simone Cesarz, Ann-Catrin Fender, Friderike Beyer, Kerttu Valtanen, Birgit Pfeiffer, Dirk Gansert, Dietrich Hertel, Andrea Polle, Rolf Daniel, Christoph Leuschner and Stefan Scheu

Published in Soil Biology and Biochemistry (2013) 61, 23-32

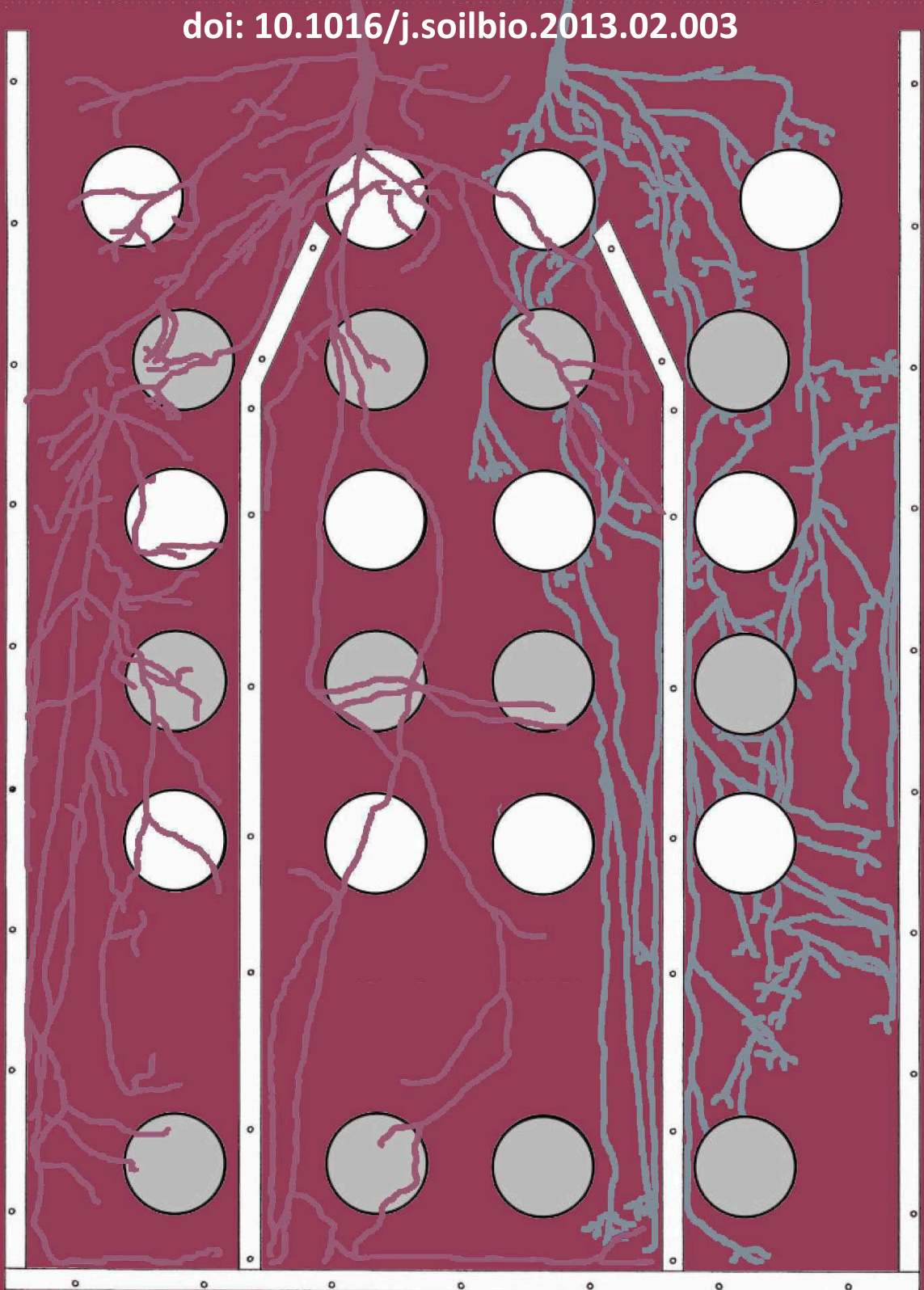




\section{Abstract}

Knowledge about the influence of living roots on de composition processes in soil is scarce but is needed to understand carbon dynamics in soil. We investigated the effect of dominant deciduous tree species of the Central European forest vegetation, European beech (Fagus sylvatica L.) and European ash (Fraxinus excelsior L.), on soil biota and carbon dynamics differentiating between root- and leaf litter-mediated effects. The influence of beech and ash seedlings on carbon and nitrogen flow was investigated using leaf litter enriched in ${ }^{13} \mathrm{C}$ and ${ }^{15} \mathrm{~N}$ in double split-root rhizotrons planted with beec $\mathrm{h}$ and ash seedlings as well as a mixture of both tree species and a control without plants. Stable isotope and compound-specific fatty acid analysis ( ${ }^{13}$ C-PLFA) were used to follow the incorporation of stable isotopes into microorganisms, soil animals and plants. Further, the bacterial community composition was analyzed using pyrosequencing of $16 \mathrm{~S}$ rRNA gene amplicons. Although beech root biomass was significantly lower than that of ash only beech significantly decreased soil carbon and nitrogen concentrations after 475 days of incubation. In addition, beech significantly decreased microbial carbon use efficiency as indicated by higher specific respiration. Low soil $\mathrm{pH}$ probably increased specific respiration of bacte ria suggesting that rhizodeposits of beech roots induced increased microbial respiration and therefore carbon loss from soil. Compared to beech $\delta^{13} \mathrm{C}$ and $\delta^{15} \mathrm{~N}$ signatures of gamasid mites in ash rhizotrons were significantly higher indicating higher amounts of litter-derived carbon and nitrogen to reach higher trophic levels. Similar $\delta^{13} \mathrm{C}$ signatures of bacteria and fine roots indicate that mainly bacteria incorporated root-derived carbon in beech rhizotrons. The result s suggest that beech and ash differentially impact soil processes with beech more strongly affecting the belowground system via root exudates and associated changes in rhizosphere microorganisms and carbon dynamics than ash. 


\section{Introduction}

Soils store twice as much carbon as plants and the atmosphere together thereby forming an important component of the global carbon cycle (Sch lesinger and Andrews, 2000). However, the way carbon is processed and how carbon dynamics are controlled still is not well understood. Knowledge on factors changing the flux of carbon from plants into the soil and controlling its turnover is of significant importance especially in face to global warming (McKinley et al., 2011).

In terrestrial ecosystems $90 \%$ of the annual biomass produced by plants enters the dead organic matter pool forming the basis of the decomposer system in soil (Gessner et al., 2010). Plant carbon enters the soil via two pathways, dead organic matter (leaf litter and dead roots) and root exudates. Soil chemical properties are mainly influenced by parent material and mineralogy but also by leaf litter forming the major resource of soil biota responsible for decomposition processes (Reich et al., 2005; Jacob et al., 2009; Langenbruch et al., 2012). Litter quality strongly influences soil $\mathrm{pH}$, as calc ium and magnesium of the litter compete with $\mathrm{H}^{+}$and $\mathrm{Al}^{3+}$ for exchange sites on soil particle surfaces or organic matter (Reich et al., 2005). As a consequence, high $\mathrm{pH}$ often promotes higher microbial biomass resulting in higher soil respiration, mineralization and decomposition (Swift et al., 1979; Wardle, 1998). Low mineralization and decomposition rates are associat ed with high C-to- $\mathrm{N}$ ratios and high lignin contents as it is typical for recalcitrant litter. In contrast, Pollierer et al. (2007) highlighted that in temperate forests carbon does not enter the soil food web predominantly via litter but rather via roots. Rhizodeposits comprise labile exu dates (e.g., sugars, amino acids and organic acids), but also complex molecules (e.g., polysaccharides, mucilage and proteins). Labile exudates control both community structure and activity of rhizosphere microorganisms 
(Paterson et al., 2009). Summarizing results of 95 plant ${ }^{14} \mathrm{C}$ labeling studies, Jones et al. (2004) estimated the loss of carbon by exudation to be equivalent to 5 - $10 \%$ of the net carbon fixed by plants and $25 \%$ of the carbon plants allocate to root growth. This supply of energy increases microbial biomass (Butler et al., 2004), acts as soil organic matter (SOM) priming agent (Bird et al., 2011) and alters the physical and chemical soil environment (Gregory, 2006). Microbial communities in rhizosphere and bulk soil are therefore responsible for root exudate-mediated changes in soil processes (Söderberg et al., 2004; Paterson et al., 2007). Since plant species differ in the quality and quantity of exudates (Jon es et al., 2004), soil carbon dynamics are likely affected by plant species identity and diversity (Grayston, Wang, et al., 1998; Steinbeiss et al., 2008).

Decomposition studies report both effects of indivi dual plant species (Jacob et al., 2009) and positive mixing effects (Gartner and Cardon, 2004; Hättenschwiler et al., 2005). Until today, however, studies investigating the influence of plant diversity on belowground dynamics in forests are scare (but see Meinen et al., 2009) and most often only consider the effect of aboveground plant residues (Hättenschwiler and Gasser, 2005; Jacob et al., 2009, 2010). To what extent belowground processes mediated by roots and root exudates affect soil organisms and thereby carbon dynamics remains largely unknown. This lack of knowledge is unfortunate as $60 \%$ of the terrestrial carbon is bound in forests and its contribution to global carbon cycling is of fundamental importance (McKinley et al., 2011).

To improve knowledge on carbon dynamics in forest soils from a root perspective we used the common temperate broad-leaved tree species European beech (Fagus sylvatica L.) and European ash (Fraxinus excelsior L.) to differentiate between general and species-specific effects of living roots on soil organisms and decom position of litter material in soil. Beech is 
the dominant tree species in many Central European deciduous forests. Ash often is associated with beech and is expected to increase in dominance in a warmer and drier climate (Broadmeadow and Ray, 2005). Life history traits of beech and ash differ strongly, e.g., speed of growth, root morphology, litter quality, mycorrhizal association, and nutrient, water and light use efficiency (Grime et al., 1997; Emborg, 1998). Beech has higher specific root tip abundance, specific fine root surface area (SRA) and specific fine root length (SRL), whereas ash roots are characterized by higher mean fine root diameter (Meinen, Hertel, et al., 2009). Roots of beech are colonized by ectomycorrhizal (EM) fungi and those of ash by arbuscular mycorrhizal (AM) fungi which differ in nutrient acquisition strategies (Smith and Read, 2008). Beech tolerates soil pH from acid to highly alkaline, while ash is restricted to soils of high base saturation (Weber-Blaschke et al., 2002). Litter of beech at more acidic sites has high C-to-N ratio ( $>50)$ and high lignin content, while ash litt er is regarded as high quality litter due to its low C-to-N ratio of about 28 and low lignin content (Jacob et al., 2010).

For allowing access to the root system and to investigate interactions between both tree species, beech and ash seedlings were planted into double split-root systems. The systems allowed dissecting root associated processes and belowground interactions between beech and ash. Carbon and nitrogen fluxes in soil were traced following the incorporation of ${ }^{13} \mathrm{C}$ and ${ }^{15} \mathrm{~N}$ from labelled ash litter into soil, bacteria, fungi, soil animals and plants. Ash litter was used to follow the uptake of resources from high quality litter materials by beech and ash as compared to more recalcitrant soil resources.

We hypothesized that (1) beech and ash differentially affect the structure of the microbial community thereby modifying soil processes and plant nutrient capture. Differences in microbial community structure are expected to (2) result in differential decomposition of 
labeled ash litter and differential mobilization of nutrients from the litter. Further, we expected (3) modifications of the soil microorganis m community and soil processes to be most pronounced in the mixed treatment with both tree species present due to complementary effects of the two tree species.

\section{Material and methods}

\section{Rhizotrons}

Double split-root rhizotrons were used to separate root systems of two tree seedlings into compartments with root strands of one individual se edling at each side and a shared root compartment in the center where root strands of both tree seedlings could interact (Fig. 1). We focused on the middle compartment where the two root strands grew together. The central compartment had a volume of $7.6 \mathrm{IH}$ and side compartments half the volume. Rhizotrons were $90 \mathrm{~cm}$ high and $64 \mathrm{~cm}$ wide, and were built from anodized aluminum covered at the front with a $10-\mathrm{mm}$ Perspex plate. They were tilted at $35^{\circ}$ to direct roots growing along the Perspex plate. The Perspex plate was covered with black scrim to ensure that roots grow in darkness. Rhizotrons were divided into six soil depth sections (I-VI). Each soil depth contained four experimental sites (ES), two in the center and two at the sides (Fig. 1). The back side of the rhizotrons was equipped with a cooling system keeping the temperature at a constant level of $20^{\circ} \mathrm{C}$ over the whole soil column. Climate conditions were set to $20^{\circ} \mathrm{C}$ air temperature, $70 \%$ relative air humidity and $10 \mathrm{~h}$ daylight in winter and $14 \mathrm{~h}$ in summer. The 
a)

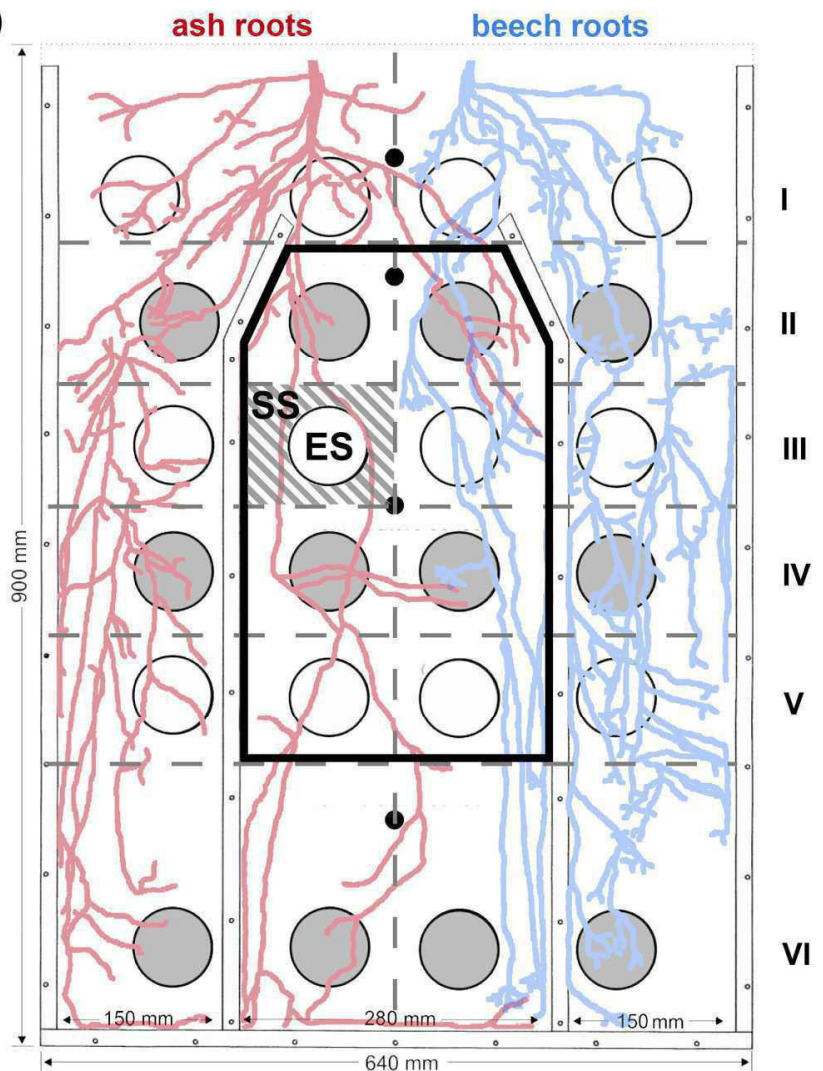

b)

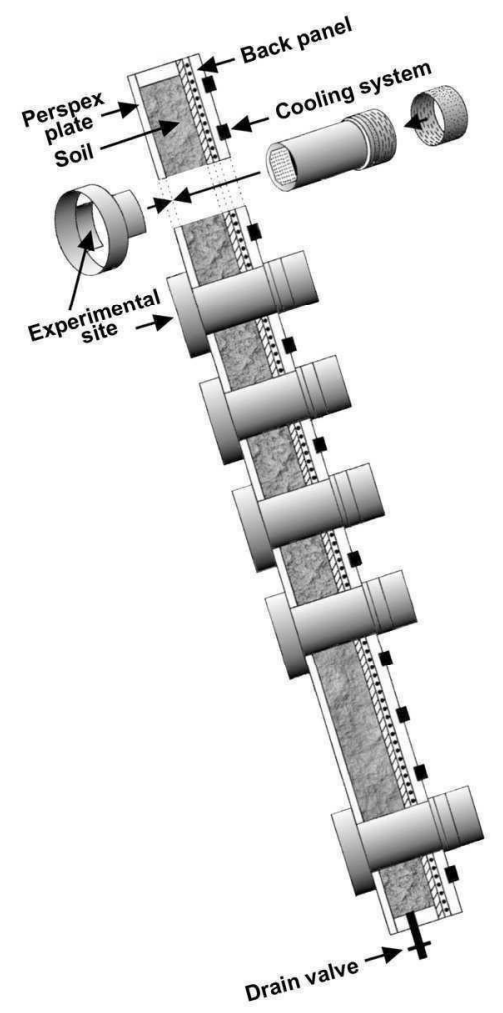

Figure 3 | Scheme of double split-root rhizotrons. (a) Front view of mixed species tr eatments with ash (left) and beech (right) roots interacting in the central compartment. Circles represent experimental sites (ES) with soil (open circles) or soil-litter mixture (grey circles). The shaded area refers to the surrounding sampling site (SS). Roman numerals indicate soil depths (I-VI). The bold rim in the central compartment from soil depth II to VI represents the sampling area. Black dots along the central dashed line refer to the position of temperature sensors. Dashed lines mark the sampling grid. (b) Side view of the double split- root rhizotron and assembly of ES. Tubes inside ES were withdrawn and the empty space filled with soil or soil-litter mixture allowing roots to grow into ES. A water flux based cooling system is insta lled at the back panel. A valve allowed drainage of the rhizotrons.

tree seedlings were illuminated (EYE Lighting, Clean Ace, Mentor, OH, USA) ensuring a minimum PPFD of $200 \pm 10 \mu \mathrm{mol} \mathrm{m} \mathrm{m}^{-2} \mathrm{~s}^{-1}$ from June 2009 to October 2010. The experiment lasted for 475 days, i.e., plants were harvested after the second season.

\section{Soil and plants}

The soil was taken from a mixed temperate broadleaf forest dominated by $F$. sylvatica, $F$. excelsior and Tilia cordata in Central Germany (Hainich forest, 51 $04^{\prime} \mathrm{N} 10^{\circ} 30^{\prime} \mathrm{E}$, about $350 \mathrm{~m}$ a.s.I.) from a depth of $0-10 \mathrm{~cm}$ after removing the litter. The soil type was a Stagnic Luvisol 
(IUSS Working Group WRB 2007; $1.8 \%$ sand, $80.2 \%$ silt and $18.1 \%$ clay) and free of carbonate $\left(<0.02 \%\right.$ of total carbon) with a $\mathrm{pH}\left(\mathrm{H}_{2} \mathrm{O}\right)$ of $4.56 \pm 0.03$ and a gravimetric water content at date of sampling of $22.7 \%$. Initial total carbon amounted to $19.2 \pm 0.3 \mathrm{~g} \mathrm{~kg}^{-1} \mathrm{dry}$ weight, initial total nitrogen averaged $1.56 \pm 0.01 \mathrm{~g} \mathrm{~kg}^{-1}$ dry weight and base saturation was $22.9 \pm 1.3 \%$. Each rhizotron was filled with $15.2 \mathrm{~L}$ of sieved soil ( $1 \mathrm{~cm}$ mesh) containing soil microflora and fauna. Volumetric soil water content was monitored three times a week with a TDR measurement device (Trime-FM, IMKO, Ettlingen, Germany), and kept at constant level by adding distilled water. Soil temperature was measured with NTC thermistors (Epcos, Munich, Germany), arranged vertically in the center of the rhizotrons at soil depths of $8,20,42.5$ and $70.5 \mathrm{~cm}$ at a distance of $2 \mathrm{~cm}$ from the Perspex plate. Data were recorded in 15-min intervals with a CR1000 data logger (combined with two AM416 Relay Multiplexer, Campbell Scientific Inc., Utah, USA). In spring 2009 beech (F. sylvatica) and ash (F. excelsior) seedlings with comparable root biomass were excavated in the Hainich forest with intact soil cores to preserve the root system. Initial shoot height was $23.1 \pm 1.2$ and $17.9 \pm 1.1 \mathrm{~cm}$, and root length was $12.1 \pm 0.7$ and $15.4 \pm 1.2 \mathrm{~cm}$ for beech and ash seedlings, respectively. At the start of the experiment, ash had significantly higher fine root biomass than beech, but tree species did not differ significantly in total root and total aboveground b iomass (Table 1). Before planting, the soil material adhering to the root systems was removed by watering. The remaining soil-water mixture was used to equilibrate microbial communiti es in soil. Fifty-three days after planting, $1.5 \mathrm{~g}$ labeled ash litter was added to ES of each of the treatments, i.e., the control, beech, ash and mixed rhizotrons at every second soil depth (II, IV, VI; see Fig. 1). Therefore, tubes were withdrawn and the empty space filled with soil or soil-litter mixture. Prior to adding ash leaves (air dried, crushed to pieces $<1 \mathrm{~cm}$ ) were mixed with $40 \mathrm{~g}$ soil (air dried). The litter was labeled 
Table $1 \mid$ Means \pm 1 SE and $T$ - and $P$-values of plant biomass of beech and ash seedlings at the start of the experiment ( $g$ dry weight plant ${ }^{-1} ; n=5$ ).

\begin{tabular}{|c|c|c|c|c|c|c|c|c|}
\hline \multirow{4}{*}{ Biomass [g dw] per plant } & \multicolumn{8}{|c|}{ Initial Biomass } \\
\hline & \multicolumn{3}{|l|}{ Beech } & \multicolumn{3}{|l|}{ Ash } & \multirow{3}{*}{$T$} & \multirow[b]{2}{*}{$\boldsymbol{P}$} \\
\hline & Mean & & SE & Mean & & SE & & \\
\hline & & & & & & & & \\
\hline Total & 2.04 & \pm & 0.46 & 2.13 & \pm & 0.22 & 0.15 & 0.7122 \\
\hline Total aboveground & 1.26 & \pm & 0.27 & 1.25 & \pm & 0.15 & 0.01 & 0.9294 \\
\hline Total belowground & 0.78 & \pm & 0.20 & 0.88 & \pm & 0.08 & 0.81 & 0.3933 \\
\hline Shoots & 0.88 & \pm & 0.22 & 0.74 & \pm & 0.09 & 0.27 & 0.6190 \\
\hline Leaves & 0.38 & \pm & 0.05 & 0.52 & \pm & 0.07 & 2.49 & 0.1530 \\
\hline Fine roots & 0.16 & \pm & 0.05 & 0.41 & \pm & 0.08 & 6.49 & 0.0343 \\
\hline Coarse roots & 0.64 & \pm & 0.17 & 0.56 & \pm & 0.06 & 0.08 & 0.7866 \\
\hline
\end{tabular}

with ${ }^{13} \mathrm{C}$ and ${ }^{15} \mathrm{~N}$ by incubating ash trees in the greenhouse for one vegetation period with the $\mathrm{CO}_{2}$ concentration in air elevated by adding ${ }^{13} \mathrm{CO}_{2}$ (maximum concentration 1,200 ppm) and by watering the soil with nutrient solution contain ing ${ }^{15} \mathrm{NO}_{3}{ }^{15} \mathrm{NH}_{4}$ (both 99 atom \%; Eurisotop, Saint-Aubin, Essonne, France). The solution co ntained $0.6 \mathrm{mM} \mathrm{CaCl}_{2}, 0.4 \mathrm{mM} \mathrm{MgSO}_{4}, 0.01$ $\mathrm{mM} \mathrm{FeCl}_{3}, 0.4 \mathrm{mM} \mathrm{K}_{3} \mathrm{PO}_{4}, 1.8 \mu \mathrm{M} \mathrm{MnSO}_{4}, 0.064 \mu \mathrm{M} \mathrm{CuCl}, 0.15 \mu \mathrm{M} \mathrm{ZnCl}_{2}, 0.1 \mu \mathrm{M} \mathrm{MoO}_{3}, 5 \mathrm{mM}$ $\mathrm{NO}_{3} \mathrm{NH}_{4}$ and $0.01 \mathrm{mM} \mathrm{H}_{3} \mathrm{BO}_{3}$. The stable isotope signature of the ash litter was $146.8 \pm 0.3 \%$ o for $\delta^{13} \mathrm{C}$ and $13,139 \pm 60 \%$ for $\delta^{15} \mathrm{~N}$ (Table 2, see Table S1 for atom\% values).

\section{Experimental design}

The experiment was set up in a factorial design with the factors beech (absence and presence) and ash (absence and presence), resulting in the following treatments with four replicates each: (a) two beech seedlings (BB), (b) two ash seedlings (AA), (c) a mixture with one beech and one ash seedling ( $B A$ or $A B$, depending on target tree species), and (d) an unplanted control $(\mathrm{Co})$, resulting in rhizotrons without ( $\mathrm{B}-\mathrm{-}$ : $\mathrm{Co}$ and $\mathrm{AA})$ and with beech ( $\mathrm{B}+\mathrm{BB}$ and $\mathrm{BA})$, as well as rhizotrons without ( $A-$ : $C o$ and $B B)$ and with ash ( $A+$ : $A A$ and $A B)$. 
Table 2 | Isotopic signatures of the soil, labeled ash litter and soil-litter-mixture at the start and end of the experiment after 422 days [(means $\pm 1 \mathrm{SE} ; \mathrm{n}=5$ except for samples taken at the end of the experiment which are pooled across treatments $(n=16)]$.

\begin{tabular}{|c|c|c|c|c|c|c|c|c|}
\hline & Start & & & & & & End & \\
\hline & Soil & & Litter & & Soil-litte & $\mathrm{r}$ mixture & Soil-lit & er mixture \\
\hline & Mean & SE & Mean & SE & Mean & SE & Mean & SE \\
\hline$\delta^{13} \mathrm{C}$ & -26.20 & \pm 0.10 & 146.80 & \pm 0.32 & 69.00 & \pm 0.60 & -17.44 & \pm 1.86 \\
\hline$\delta^{15} N$ & 1.60 & \pm 0.16 & 13139.30 & \pm 59.10 & 6153.80 & \pm 0.40 & 577.38 & \pm 124.88 \\
\hline
\end{tabular}

\section{Sampling}

After 475 days, rhizotrons were harvested. They were opened in horizontal position and a sampling grid was used to identify locations for sa mpling, i.e., at ES and the surrounding of these sites (SS; see Fig. 1). Samples from the depth layers II, III, IV and V of the central compartment were analyzed. As we were not interested in effects of soil depth we pooled the data from the four layers. In addition to soil samples, plant shoots and roots from each of the soil layers were taken for measuring plant biomass. Details on root biomass distribution along the soil depth gradient as well as on gas emissions are presented elsewhere (Fender et al., 2013)

\section{Plants}

At harvest shoot length and root collar diameter of seedlings were measured. Roots were separated from soil, washed and cleaned from adhering soil particles. To obtain overall plant biomass fine root biomass estimated from ES for mycorrhizal analysis were combined with plant biomass data from SS. Whenever possible three intact root strands of ca $7 \mathrm{~cm}$ length from each tree species per compartment and soil depths were taken and digitized on a flatbed scanner for image analysis carried out using WinRhizo 2005c software (Régent Instruments Inc., Québec, QC, Canada) to determine specific fine root area (SRA; $\mathrm{cm}^{2} \mathrm{~g}^{-1} \mathrm{dry}$ matter), specific fine root length (SRL; $\mathrm{cm} \mathrm{g}^{-1}$ dry matter) and total fine root surface. Thereafter, 
samples were oven-dried $\left(70^{\circ} \mathrm{C}, 48 \mathrm{~h}\right)$, weighed and milled for measurement of organic carbon $\left(\mathrm{C}_{\text {org }}\right)$, total nitrogen ( $\mathrm{N}_{\text {total }}$ ) as well as $\delta^{13} \mathrm{C}$ and $\delta^{15} \mathrm{~N}$ signatures (Delta $\mathrm{C}$, Finnigan MAT, Bremen, Germany).

\section{Mycorrhiza}

Colonization of roots at ES by mycorrhiza-forming fungi was determined. Fine roots were stored in Falcon tubes with moist tissue paper at $4{ }^{\circ} \mathrm{C}$ until analysis. Fine roots of beech were analyzed with a stereomicroscope (Leica M205 FA, Leica Microsystems, Wetzlar, Germany). The percentage of EM fungi colonization was calcula ted using the following equation:

EM fungi colonization [\%] $=\left(\frac{n \text { mycorrhizal root tips }}{n \text { vital root tips }}\right) \times 100$

Fine roots of ash were stored in $70 \% \mathrm{EtOH}$ at room temperature. For determining the colonization by AM fungi roots were stained with la ctophenole-blue (Schmitz et al., 1991) and stored at room temperature in $50 \%$ glycerol until microscopic inspection at $200 \times$ magnification. AM fungi colonization was calculated with the magnified intersection method of McGonigle et al. (1990) using a $10 \times 10$ grid. The abundance of vesicles, arbuscles and hyphae was calculated as percentage of mycorrhizal structures of the total number of intersections. The percentage of vesicles was taken as relative colonization rate of AM fungi and used for further calculations.

\section{Soil properties}

Soil pH was measured in a suspension of $10 \mathrm{~g}$ soil and $25 \mathrm{ml} \mathrm{H}_{2} \mathrm{O}$ with a Vario $\mathrm{pH}$ meter (WTW $\mathrm{GmbH}$, Weilheim, Germany). Soil water content was measured gravimetrically after drying at $105^{\circ} \mathrm{C}$ for $24 \mathrm{~h}$. Nitrate and ammonium concentrations were measured by extracting soil samples in $0.5 \mathrm{M} \mathrm{K}_{2} \mathrm{SO}_{4}$ solution (1:3 wet soil mass-to-solution ratio). Sa mples were shaken for 
$1 \mathrm{~h}$ and filtered through Sartorius folded filters (Sartorius Stedim, Aubagne, France). Nitrate and ammonium concentrations of filtered extracts were analyzed using continuous flow injection colorimetry $\left(\mathrm{SAN}^{+}\right.$Continuous Flow Analyzer, Skalar Instruments, Breda, Netherlands). Nitrate was determined by copper cadmium reduction method (ISO method 13395) and ammonium was quantified by Berthelot reaction method (ISO method 11732). $\mathrm{C}_{\text {org }}, \mathrm{N}_{\text {total }}$ as well as $\delta^{13} \mathrm{C}$ and $\delta^{15} \mathrm{~N}$ values were measured after grinding soil samples with a disc mill. Samples were analyzed with a coupled system consisting of an elemental analyzer (NA 1500, Carlo Erba, Mailand) and a mass spectrometer (Delta C, Finnigan MAT, Bremen, Germany).

\section{Microbial respiration}

Basal respiration (BAS), microbial biomass $\left(\mathrm{C}_{\mathrm{mic}}\right)$, and specific respiration $\left(q \mathrm{O}_{2}\right)$ were measured by substrate-induced respiration (SIR), i.e., the respiratory response of microorganisms to glucose (Anderson and Domsch, 1978). Before measurement, roots were removed and soil samples were sieved $(2 \mathrm{~mm})$. Measurements were done using an automated $\mathrm{O}_{2}$ microcompensation system (Scheu, 1992). BAS of microorganisms reflected their averaged oxygen consumption rate without the addition of glu cose within 10-30 h after attachment of the samples to the analysis system. Subsequently, $4 \mathrm{mg}$ glucose $\mathrm{g}^{-1}$ soil dry weight was added as aqueous solution to the soil samples. The mean of the three lowest hourly measurements within the first $10 \mathrm{~h}$ was taken as the maximum initial respiratory response (MIRR). $\mathrm{C}_{\text {mic }}(\mu \mathrm{g} \mathrm{C}$ $\left.\mathrm{g}^{-1}\right)$ was calculated as $38 \times \operatorname{MIRR}\left(\mu \mathrm{O}_{2} \mathrm{~g}^{-1}\right.$ soil dry weight $\left.\mathrm{h}^{-1}\right)$ according to Beck et al., (1997). Microbial specific respiration $q \mathrm{O}_{2}\left(\mu \mathrm{O}_{2} \mathrm{mg}^{-1} \mathrm{C}_{\text {mic }} \mathrm{h}^{-1}\right)$ was calculated as $\mathrm{BAS} / \mathrm{C}_{\text {mic. }}$. 


\section{Fatty acid analysis}

Before extraction of lipids, soil samples were sieved $(2 \mathrm{~mm})$ and root and litter pieces were removed. Lipid extraction followed Frostegård et al. (1991). Bacterial biomass was estimated using the following PLFAs: a15:0, i15:0, i16:0, 16:1w7, i17:0, cy17:0 and cy19:0; the PLFA 18:2w6,9 was used as fungal biomarker (Ruess and Chamberlain, 2010). A gaschromatography-combustion-isotope-ratio-monitoring-mass spectrometer (GC-C-IRM-MS) using Thermo Finnigan Trace GC coupled via a GP interface to a Delta Plus mass spectrometer (Finnigan, Bremen, Germany) was used to determine the isotopic composition of individual PLFAs. Fatty acid identification was verified by GC-MS using a Varian CP-3800 chromatograph coupled to a $1200 \mathrm{~L}$ mass spectrometer and a fused silica column (Phenomenex Zebron ZB$5 \mathrm{MS}, 30 \mathrm{~m}, 0.25 \mu \mathrm{m}$ film thickness, ID $0.32 \mathrm{~mm}$ ) and helium as carrier gas.

\section{Pyrosequencing}

$16 \mathrm{~S}$ rRNA as well as the $16 \mathrm{~S}$ rDNA were co-isolated to capture the active and the present microbial community; $2 \mathrm{~g}$ soil were extracted from control, beech and ash treatments using the RNA PowerSoil ${ }^{\mathrm{TM}}$ Total RNA Isolation Kit and DNA Elution Accessory Kit (MO BIO Laboratories Inc., Carlsbad, CA, USA). Residual DNA contaminations in RNA extracts were removed using the TURBO DNA-free ${ }^{\mathrm{TM}}$ Kit (Ambion Applied Biosystems, Darmstadt, Germany). RNA was concentrated using the RNeasy MiniElute Kit (QIAGEN, Hilden, Germany). The nucleic acid concentration was estimated using a NanoDrop ND-1000 spectrophotometer (Peqlab Biotechnologie $\mathrm{GmbH}$, Erlangen, Germany).

The V2-V3 region of the 16S rRNA was reverse transcribed using the SuperScript ${ }^{\mathrm{TM}}$ III reverse transcriptase (Invitrogen, Karlsruhe, Germany). As template $100 \mathrm{ng}$ of the DNA-free RNA were applied. The resulting cDNA as well as the extracted DNA was amplified in triplicate using the 
Phusion ${ }^{\circledR}$ Hot Start High-Fidelity DNA polymerase (FINNZYMES, Espoo, Finland) as described by Nacke et al. (2011).

The following barcoded primer set was used for reverse transcription and amplification, containing the Roche 454 pyrosequencing adaptors (underlined): V2for 5'CTATGCGCCTTGCCAGCCCGCTCAGAGTGGCGGACGGGTGAGTAA-3' and V3rev 5'CGTATCGCCTCCCTCGCGCCATCAGCGTATTACCGCGGCTGCTG-3' modified from (Schmalenberger et al., 2001).

The PCR products were treated and purified as described by Nacke et al. (2011). All kits were used as described in the manufacturer's instructions. The Göttingen Genomics Laboratory determined the sequences of the partial 16S rRNA genes using a Roche GS-FLX 454 pyrosequencer (Roche, Mannheim, Germany) according to the manufacturer's instructions for amplicon sequencing.

Sequences shorter than $300 \mathrm{bp}$ were removed from the dataset. To minimize the bias introduced by pyrosequencing due to decreasing read precision at the end of the reads denoising was carried out using Denoiser 0.91 (Reeder and Knight, 2010). OTU determination was performed using uclust OTU picker 1.2.22q (Edgar, 2010) at genetic divergence of 3\%, 5\% and $20 \%$ according to Schloss and Handelsman (2005). The resulting datasets have been deposited in the GenBank short-read archive under accession number SRA050002.

\section{Soil animals}

Soil not needed for other analysis was taken to extract soil animals by heat (Kempson et al., 1963). Animals were preserved in saturated $\mathrm{NaCl}$ solution and kept at $-10^{\circ} \mathrm{C}$ until analysis. The gamasid mite Hypoaspis aculeifer (G. Canestrini, 1884) was taken for stable isotope analysis 
as it occurred in sufficient numbers. Twenty adult mites were weighed into each tin capsule and dried at $40^{\circ} \mathrm{C}$ for $24 \mathrm{~h}$. Samples were analyzed as described above.

\section{Statistical analysis}

Two-way ANOVA was used to test for main effects of beech ( $B-$ and $B+)$, ash $(A-$ and $A+$ ) and their interactions with data of the four soil depths pooled. To detect differences in plant biomass and mycorrhizal colonization contrasts were calculated in a GLM using pairwise $t$-test to account for dependence in mixed rhizotrons. U-Test was used for analyzing the number of root tips. Treatments in beech-only rhizotrons (BB) were compared to ash-only (AA) and beech-ash mixture (BA). Ash ( $A A)$ was also compared with beech-ash mixture (AB). Statistical analyses were done using SAS 9.2 (SAS Institute; Cary, NC, USA).

Discriminant function analysis (DFA) was used to an alyze pyrosequencing data as well as fatty acid patterns combined with microbial respiration and soil chemical data. Differences of the bacterial composition in beech and ash rhizotrons a nd the control were calculated using nonmultidimensional scaling (NMDS) to reduce dimension s in the dataset. DFA and NMDS were calculated using STATISTICA 7.0 for Windows (StatSo ft, Tulsa, USA, 2001).

Means were compared using Tukey's Honestly Significant Difference test $(P<0.05)$. Data on plant biomass, isotopic signatures, SRA, SLR, number of fine root tips, $\mathrm{NO}_{3}{ }^{-}, \mathrm{NH}^{+}, \mathrm{C}_{\text {org }}, \mathrm{N}_{\text {total }}$, microbial respiration and PLFA content were log-transformed and percentage data, i.e., colonization rate of mycorrhiza, were arcsine-square root transformed prior to statistical analyses to improve homogeneity of variance. Means given in text and tables are based on non-transformed data. 


\section{Results}

\section{Plants and mycorrhizae}

After 475 days, total biomass of tree seedlings in BB rhizotrons was significantly lower than in $A A$ and $B A$ rhizotrons (Table 3). Fine and coarse root biomass were significantly lower in BB rhizotrons compared to that of seedlings in $A A(-69 \%)$ and $B A$ rhizotrons $(-62 \%)$ resulting in significantly lower total root biomass. Total bioma ss, total root biomass and coarse root biomass of seedlings in mixtures exceeded that of seedlings in monocultures, but this increase was only significant for beech $(60 \%, 62 \%, 70 \%$, respectively); biomass of ash seedlings in mixture increased by $11 \%, 17 \%$ and $23 \%$, respectively.

$\delta^{13} \mathrm{C}$ and $\delta^{15} \mathrm{~N}$ signatures in fine roots were significantly lower in BB than those in AA rhizotrons (Table 3; see Table S2 for atom\% values). SRA and SRL did not differ significantly between tree species but tended to be higher in beech (BB vs $A A:+6 \%$ and $+68 \%$, respectively), especially in the mixture (BA vs $A B:+24 \%$ and $+79 \%$, respectively). Generally, fine root tips of tree seedlings increased in mixed rhizotrons, especially beech in mixed rhizotrons had a significantly higher number of root tips than beech in monoculture by $+89 \%$ compared to ash in mixed rhizotrons and by $+54 \%$ compared to ash in monoculture. Mycorrhizal colonization of roots of beech in $B B$ rhizotrons was significantly lower than that of roots of ash in AA rhizotrons, however, as beech and ash are colonized by different types of mycorrhiza the differences have to be interpreted with caution. Beech did not influence the colonization rate of ash by arbuscular mycorrhiza ( $A A$ vs $A B ;+2 \%$ ), whereas ash increased the colonization of beech by ectomycorrhiza (BB vs BA; +45\%) although the effect was not significant (Table 3). 
Table 3 | GLM table of contrasts between rhizotrons planted with beech (BB), ash (AA), beech mixed with ash (BA) and ash mixed with beech (AB) for plant parameters of rhizotrons planted with beech, ash or both after 475 days as well as means \pm 1 SE of the respective parameters ( $n=4)$. Significant effects are given in bold. Atom\% values of plant compartments are given in Table S2.

\begin{tabular}{|c|c|c|c|c|c|c|c|c|c|c|c|c|c|c|c|c|c|c|}
\hline \multirow[b]{3}{*}{ Biomass [g dw] per plant } & \multicolumn{2}{|c|}{ BB vs $A A$} & \multicolumn{2}{|c|}{ BB vs BA } & \multicolumn{2}{|c|}{$A A$ vs $A B$} & \multicolumn{3}{|c|}{$\begin{array}{c}\text { BB } \\
\text { (pure beech) }\end{array}$} & \multicolumn{3}{|c|}{$\begin{array}{c}\mathbf{A A} \\
\text { (pure ash) }\end{array}$} & \multicolumn{3}{|c|}{$\begin{array}{c}\text { BA } \\
\text { (beech in mixture) } \\
\end{array}$} & \multicolumn{3}{|c|}{$\begin{array}{c}\mathbf{A B} \\
\text { (ash in mixture) }\end{array}$} \\
\hline & $\boldsymbol{F}$ & $P$ & $\boldsymbol{F}$ & $P$ & $F$ & $P$ & Mean & & SE & Mean & & SE & Mean & & SE & Mean & & SE \\
\hline & & & & & & & & & & & & & & & & & & \\
\hline Total & 8.82 & 0.0117 & 6.39 & 0.0266 & 0.00 & 0.9518 & 4.52 & \pm & 0.79 & 12.08 & \pm & 1.69 & 11.33 & \pm 2 & 2.03 & 13.50 & \pm & 2.33 \\
\hline Total aboveground & 4.20 & 0.0629 & 2.91 & 0.1138 & 1.11 & 0.3128 & 1.85 & \pm & 0.38 & 3.34 & \pm & 0.86 & 4.29 & \pm & 1.01 & 3.03 & \pm & 0.43 \\
\hline Total root & 9.52 & 0.0094 & 6.96 & 0.0217 & 0.28 & 0.6035 & 2.67 & \pm & 0.50 & 8.74 & \pm & 1.08 & 7.04 & \pm 1 & 1.08 & 10.47 & \pm & 2.15 \\
\hline Shoot & 1.24 & 0.2876 & 3.43 & 0.0889 & 0.12 & 0.7300 & 1.38 & \pm & 0.25 & 1.78 & \pm & 0.32 & 3.20 & \pm & 0.73 & 2.64 & \pm & 0.41 \\
\hline Leaves & 5.14 & 0.0426 & 0.53 & 0.4809 & 7.50 & 0.0180 & 0.46 & \pm & 0.14 & 1.56 & \pm & 0.56 & 1.08 & \pm & 0.34 & 0.39 & \pm & 0.22 \\
\hline Fine roots & 9.14 & 0.0106 & 4.60 & 0.0532 & 0.09 & 0.7669 & 0.78 & \pm & 0.18 & 2.38 & \pm & 0.30 & 1.80 & \pm & 0.27 & 2.27 & \pm & 0.44 \\
\hline Coarse roots & 7.95 & 0.0154 & 6.50 & 0.0255 & 0.59 & 0.4557 & 1.89 & \pm & 0.35 & 6.36 & \pm & 0.87 & 5.24 & \pm & 0.82 & 8.21 & \pm & 1.91 \\
\hline \multicolumn{19}{|c|}{$\delta^{13} \mathrm{C}[\%$ ] plant compartments } \\
\hline Shoot & 5.14 & 0.0426 & 7.00 & 0.0214 & 2.12 & 0.1708 & -29.09 & \pm & 0.32 & -28.07 & \pm & 0.28 & -27.90 & \pm & 0.22 & -27.40 & \pm & 0.26 \\
\hline Leaves & 0.30 & 0.5955 & 0.25 & 0.6287 & 0.75 & 0.4029 & -29.62 & \pm & 0.56 & -29.26 & \pm & 0.27 & -29.29 & \pm & 0.44 & -29.83 & \pm & 0.20 \\
\hline Fine roots & 8.27 & 0.0139 & 0.04 & 0.8402 & 0.01 & 0.9395 & -27.64 & \pm & 0.34 & -25.60 & \pm & 0.85 & -27.49 & \pm & 0.19 & -25.56 & \pm & 0.23 \\
\hline Coarse roots & 12.86 & 0.0037 & 2.78 & 0.1215 & 0.06 & 0.8162 & -28.35 & \pm & 0.31 & -25.74 & \pm & 0.76 & -27.15 & \pm & 0.31 & -25.92 & \pm & 0.32 \\
\hline \multicolumn{19}{|c|}{$\delta^{15} \mathrm{~N}[\%$ ] plant compartments } \\
\hline Shoot & 0.87 & 0.3701 & 0.07 & 0.8018 & 2.15 & 0.1682 & 171.27 & \pm & 30.67 & 260.05 & \pm & 66.16 & 154.54 & \pm 1 & 18.34 & 154.40 & \pm & 26.76 \\
\hline Leaves & 5.34 & 0.0394 & 0.55 & 0.4741 & 1.98 & 0.1853 & 192.42 & \pm & 32.67 & 316.50 & \pm & 43.37 & 166.67 & \pm 2 & 23.49 & 228.28 & \pm & 15.10 \\
\hline Fine roots & 4.77 & 0.0496 & 1.35 & 0.2674 & 4.07 & 0.0666 & 209.02 & \pm & 41.75 & 396.07 & \pm & 99.34 & 148.85 & \pm & 17.63 & 214.48 & \pm & 22.80 \\
\hline Coarse roots & 9.34 & 0.0100 & 0.10 & 0.7630 & 2.81 & 0.1196 & 193.66 & \pm & 27.78 & 390.78 & \pm & 78.87 & 178.50 & \pm & 12.60 & 257.86 & \pm & 19.23 \\
\hline \multicolumn{19}{|l|}{$\mathrm{SRA}^{\S}\left[\mathrm{m}^{2} / \mathrm{g}\right]$} \\
\hline $\begin{array}{l}\text { Fine roots } \\
\text { SRL }^{\S}[\mathrm{m} / \mathrm{g}]\end{array}$ & 0.23 & 0.6385 & 0.05 & 0.8271 & 0.78 & 0.3950 & 485.16 & \pm & 15.36 & 456.49 & \pm & 42.70 & 509.00 & \pm 5 & 54.07 & 410.65 & \pm & 64.00 \\
\hline Fine roots & 2.89 & 0.1150 & 0.50 & 0.4947 & 0.20 & 0.6596 & 2374.80 & \pm & 221.17 & 1414.42 & \pm & 168.82 & 3235.44 & $\pm \varepsilon$ & 848.14 & 1810.83 & \pm & 450.85 \\
\hline \multicolumn{19}{|l|}{ Fine root tips } \\
\hline $\begin{array}{c}\text { Number } \\
\text { Mycorrhiza [\%] }\end{array}$ & -0.48 & 0.9970 & -13.16 & 0.0000 & 2.13 & 0.1750 & 1623.50 & \pm & 230.01 & 2299.00 & \pm & 419.58 & 3072.50 & \pm 2 & 207.37 & 3543.75 & \pm & 107.79 \\
\hline Colonization $^{\dagger}$ & 27.50 & 0.0002 & 3.07 & 0.1053 & 0.04 & 0.8481 & 37.81 & \pm & 8.58 & 81.82 & \pm & 5.17 & 54.80 & \pm & 6.51 & 83.54 & \pm & 2.87 \\
\hline
\end{tabular}

${ }^{\dagger}$ Note that the different type of mycorrhiza in beech and ash demanded for special counting techniques, thus direct comparisons have to be treated with caution but allow comparison with trees in mixture. 
Table $4 \mid$ ANOVA table of $F$ - and $P$-values on the effect of beech and ash on soil and microbial parameters, and signatures in ga masid mites as well as means \pm 1 SE of the respective parameters in rhizotrons planted with beech (B) and ash (A) after 475 days $(n=4)$. Significant effects are given in bold. Atom\% values of soil C and N, PLFA and gamasid mites are given in Table S2.

\begin{tabular}{|c|c|c|c|c|c|c|c|c|c|c|c|c|c|c|c|c|c|c|}
\hline \multirow[b]{4}{*}{ Soil data } & \multirow{2}{*}{\multicolumn{2}{|c|}{ Beech }} & \multirow{2}{*}{\multicolumn{2}{|c|}{ Ash }} & \multirow{2}{*}{\multicolumn{2}{|c|}{ Beech $\times$ Ash }} & \multicolumn{6}{|c|}{ B- } & \multicolumn{6}{|c|}{$B+$} \\
\hline & & & & & & & (Co & $\begin{array}{l}\text { A- } \\
\text { ntro }\end{array}$ & & & Ash) & & & eech & & (Mix & ur & \\
\hline & \multirow[t]{2}{*}{$\mathbf{F}$} & \multirow[t]{2}{*}{$\mathbf{P}$} & \multirow[t]{2}{*}{$\mathbf{F}$} & \multirow[t]{2}{*}{$\mathbf{P}$} & \multirow[t]{2}{*}{$\mathbf{F}$} & \multirow[t]{2}{*}{$\mathbf{P}$} & \multirow[t]{2}{*}{ Mean } & \multirow{2}{*}{\multicolumn{2}{|c|}{ SE }} & \multirow[t]{2}{*}{ Mean } & \multirow{2}{*}{\multicolumn{2}{|c|}{ SE }} & \multirow[t]{2}{*}{ Mean } & \multirow{2}{*}{\multicolumn{2}{|c|}{ SE }} & \multirow[t]{2}{*}{ Mean } & \multirow{2}{*}{\multicolumn{2}{|c|}{ SE }} \\
\hline & & & & & & & & & & & & & & & & & & \\
\hline $\mathrm{pH}\left(\mathrm{H}_{2} \mathrm{O}\right)$ & 5.77 & 0.0334 & 0.11 & 0.7436 & 0.02 & 0.8944 & 4.78 & \pm & 0.12 & 4.83 & \pm & 0.05 & 4.53 & \pm & 0.14 & 4.55 & \pm & 0.11 \\
\hline $\mathrm{N}-\mathrm{NO}_{3}{ }^{-}\left[\mathrm{mg} \mathrm{kg}^{-1} \mathrm{dw}\right]$ & 1.00 & 0.3387 & 0.04 & 0.8532 & 0.62 & 0.4487 & 41.31 & \pm & 4.96 & 42.88 & \pm & 3.20 & 39.32 & \pm & 3.83 & 35.14 & \pm & 5.93 \\
\hline $\mathrm{N}-\mathrm{NH}_{4}{ }^{+}\left[\mathrm{mg} \mathrm{kg}^{-1} \mathrm{dw}\right]$ & 0.01 & 0.9422 & 0.41 & 0.5360 & 0.38 & 0.5477 & 2.46 & \pm & 0.93 & 1.47 & \pm & 0.63 & 1.88 & \pm & 0.72 & 1.86 & \pm & 0.69 \\
\hline$C_{\text {org }}\left[\mathrm{mg} \mathrm{kg}^{-1} \mathrm{dw}\right]$ & 15.02 & 0.0022 & 0.08 & 0.7829 & 0.02 & 0.8980 & 1.89 & \pm & 0.04 & 1.91 & \pm & 0.05 & 1.76 & \pm & 0.03 & 1.77 & \pm & 0.02 \\
\hline$\delta^{13} \mathrm{C}$ soil [\%o] & 7.54 & 0.0177 & 1.73 & 0.2129 & 1.40 & 0.2604 & -23.27 & \pm & 0.58 & -21.21 & \pm & 1.35 & -24.51 & \pm & 0.57 & -24.41 & \pm & 0.40 \\
\hline $\mathrm{N}_{\text {total }}\left[\mathrm{mg} \mathrm{kg}^{-1} \mathrm{dw}\right]$ & 7.82 & 0.0162 & 0.24 & 0.6297 & 0.00 & 0.9687 & 0.18 & \pm & 0.00 & 0.17 & \pm & 0.00 & 0.17 & \pm & 0.00 & 0.16 & \pm & 0.00 \\
\hline$\delta^{15} \mathrm{~N}$ soil $[\%$ o] & 7.42 & 0.0185 & 0.83 & 0.3816 & 0.31 & 0.5907 & 212.18 & \pm & 55.44 & 318.33 & \pm & 78.47 & 126.29 & \pm & 37.64 & 127.79 & \pm & 20.99 \\
\hline C-to-N ratio & 0.56 & 0.4677 & 0.98 & 0.3406 & 0.00 & 0.9932 & 10.78 & \pm & 0.24 & 10.94 & \pm & 0.08 & 10.66 & \pm & 0.16 & 10.82 & \pm & 0.14 \\
\hline $\mathrm{CEC}\left[\mu \mathrm{molc} \mathrm{g}^{-1} \mathrm{dw}\right]$ & 0.06 & 0.8162 & 1.33 & 0.2726 & 0.06 & 0.8109 & 189.78 & \pm & 3.98 & 185.12 & \pm & 2.33 & 191.98 & \pm & 9.94 & 201.36 & \pm & 7.84 \\
\hline Base saturation [\%] & 1.39 & 0.2638 & 0.04 & 0.8518 & 1.13 & 0.3108 & 20.21 & \pm & 0.29 & 20.80 & \pm & 0.65 & 19.90 & \pm & 0.99 & 20.92 & \pm & 0.47 \\
\hline \multicolumn{19}{|l|}{ Microbial parameters } \\
\hline $\operatorname{BAS}\left[\mu l O_{2} h^{-1} g^{-1}\right]^{\S}$ & 4.04 & 0.0674 & 0.09 & 0.7674 & 0.19 & 0.6701 & 1.18 & \pm & 0.09 & 1.18 & \pm & 0.05 & 1.41 & \pm & 0.07 & 1.36 & \pm & 0.15 \\
\hline$C_{\text {mic }}\left[\mu g \mathrm{Cg}^{-1}\right]^{\S}$ & 0.03 & 0.8643 & 0.48 & 0.5019 & 0.40 & 0.5365 & 150.03 & \pm & 13.65 & 134.32 & \pm & 5.93 & 139.79 & \pm & 6.62 & 140.86 & \pm & 13.38 \\
\hline$q \mathrm{O}_{2}\left[\mu \mathrm{l} \mathrm{O} \mathrm{mg}^{-1} \mathrm{C}_{\mathrm{mic}} \mathrm{h}^{-1}\right]^{\S}$ & 9.00 & 0.0111 & 0.14 & 0.7178 & 1.59 & 0.2311 & 0.008 & \pm & 0.001 & 0.009 & \pm & 0.000 & 0.010 & \pm & 0.001 & 0.010 & \pm & 0.001 \\
\hline \multicolumn{19}{|l|}{ PLFA [nmol g ${ }^{-1}$ dry weight] } \\
\hline Total & 0.75 & 0.4025 & 0.00 & 0.9619 & 1.11 & 0.3130 & 7.22 & \pm & 1.32 & 6.03 & \pm & 1.36 & 6.57 & \pm & 0.55 & 8.19 & \pm & 0.97 \\
\hline Bacteria & 0.53 & 0.4801 & 0.01 & 0.9377 & 1.05 & 0.3262 & 6.95 & \pm & 1.20 & 5.85 & \pm & 1.29 & 6.25 & \pm & 0.52 & 7.66 & \pm & 0.95 \\
\hline Fungi & 3.36 & 0.0916 & 0.18 & 0.6757 & 1.20 & 0.2955 & 0.27 & \pm & 0.16 & 0.18 & \pm & 0.07 & 0.33 & \pm & 0.05 & 0.53 & \pm & 0.15 \\
\hline Fungi-to-bacteria ratio & 5.17 & 0.0422 & 0.33 & 0.5755 & 0.85 & 0.3752 & 0.032 & \pm & 0.017 & 0.026 & \pm & 0.010 & 0.050 & \pm & 0.008 & 0.073 & \pm & 0.019 \\
\hline \multicolumn{19}{|l|}{ PLFA $\delta^{13} \mathrm{C}[\% \circ]$} \\
\hline Total & 2.43 & 0.1454 & 1.40 & 0.2590 & 0.30 & 0.5944 & -22.80 & \pm & 2.37 & -21.49 & \pm & 2.09 & -27.14 & \pm & 0.60 & -23.55 & \pm & 2.51 \\
\hline Bacteria & 2.01 & 0.1818 & 0.49 & 0.4960 & 0.51 & 0.4871 & -24.38 & \pm & 1.47 & -24.43 & \pm & 1.05 & -27.25 & \pm & 0.45 & -25.31 & \pm & 1.89 \\
\hline Fungi & 7.48 & 0.0181 & 0.08 & 0.7807 & 0.16 & 0.6941 & -21.01 & \pm & 6.61 & -17.06 & \pm & 4.53 & -31.59 & \pm & 0.92 & -28.27 & \pm & 4.01 \\
\hline \multicolumn{19}{|l|}{ Gamasid mites } \\
\hline$\delta^{13} \mathrm{C}[\%$ o] & 20.59 & 0.0008 & 159.43 & $<.0001$ & 7.80 & 0.0175 & -23.37 & \pm & 0.86 & -13.89 & \pm & 0.31 & -20.19 & \pm & 1.40 & -8.78 & \pm & 0.43 \\
\hline$\delta^{15} \mathrm{~N}[\%$ ] & 25.75 & 0.0004 & 148.88 & $<.0001$ & 11.93 & 0.0054 & 130.14 & \pm & 23.08 & 713.33 & \pm & 43.37 & 339.07 & \pm & 37.35 & 1121.26 & \pm & 26.97 \\
\hline
\end{tabular}

${ }^{\S} \mathrm{BAS}$, basal respiration; $\mathrm{C}_{\text {mic, }}$ microbial biomass; $\mathrm{qO}_{2}$, specific respiration. 


\section{Soil properties}

In general, the studied soil properties were strongly affected by beech and not by ash with interactions between tree species also being not significant (Table 4). Soil pH was significantly lower in $\mathrm{B}+(4.54 \pm 0.08)$ than in $\mathrm{B}$ - rhizotrons $(4.80 \pm 0.06)$. In presence of beech $\mathrm{C}_{\text {org }}$ and $\mathrm{N}_{\text {total }}$ were significantly decreased by $-7 \%$ and $-6 \%$, respectively, but $\mathrm{NO}_{3}{ }^{-}$and $\mathrm{NH}_{4}{ }^{+}$concentrations remained unaffected (Table 4, see Table S3 for atom\% values). Further, $\delta^{13} \mathrm{C}$ and $\delta^{15} \mathrm{~N}$ of bulk soil were significantly lower in $B+(-24.46 \pm 0.32 \%$ ond $127.04 \pm 19.95 \%$, respectively $)$ compared to B- rhizotrons (-22.24 $\pm 0.78 \%$ o and $265.25 \pm 48.79 \%$, respectively). Generally, after 422 days of litter incubation, the signatures of $\delta^{13} \mathrm{C}$ and $\delta^{15} \mathrm{~N}$ within the soil-littermixtures decreased strongly by 86 and 5576 delta units, respectively (Table 2; see Table S1 for atom $\%$ values).

\section{Microorganisms}

$\mathrm{C}_{\text {mic }}$ was not significantly affected by tree species and averaged over all treatments $141.25 \pm$ $4.93 \mu \mathrm{g} \mathrm{C} \mathrm{g}{ }^{-1}$. However, $q \mathrm{O}_{2}$ was significantly higher in $\mathrm{B}+\left(0.0101 \pm 0.003 \mu \mathrm{O}_{2} \mathrm{mg}^{-1} \mathrm{C}_{\text {mic }} \mathrm{h}^{-1}\right)$ than in B- rhizotrons (-16\%, Table 4), which was due to marginally higher BAS in B+ $(1.39 \pm$ $\left.0.08 \mu \mathrm{O} \mathrm{O}_{2} \mathrm{~h}^{-1} \mathrm{~g}^{-1}\right)$ as compared to B- rhizotrons (-15\%).

The ratio of fungal-to-bacterial marker PLFAs was significantly higher in $B+(0.061 \pm 0.007)$ than in $\mathrm{B}$ - rhizotrons $(-53 \%)$ as the fungal biomass was higher in $\mathrm{B}+\left(0.43 \pm 0.08 \mathrm{nmol} \mathrm{g} \mathrm{g}^{-1} \mathrm{dry}\right.$ weight) than in B- rhizotrons (-47\%), whereas bacterial biomass remained unaffected.

Bacterial and total PLFA content were not significantly affected by the treatments and averaged $6.67 \pm 1.67$ and $7.00 \pm 0.53 \mathrm{nmol} \mathrm{g}{ }^{-1}$ dry weight, respectively. The $\delta^{13} \mathrm{C}$ values of the fungal marker PLFA $18: 2 \omega 6,9$ were significantly lower in $B+(-29.93 \pm 2.00 \%$ o $)$ than in Brhizotrons $\left(-18.75 \pm 3.60 \%\right.$ o). Also, weighted $\delta^{13} \mathrm{C}$ values of bacterial PLFAs were lower in $\mathrm{B}+(-$ 
$26.28 \pm 0.97 \%$ o $)$ than in $B$ - rhizotrons $(-24.40 \pm 0.84 \%$ o), whereas in $A+$ rhizotrons $(-24.87 \pm$ $1.01 \%$ ) they tended to be higher than in A- rhizotrons $(-25.82 \pm 0.89 \%$ ). In general, ash did not significantly influence $\delta^{13} \mathrm{C}$ values of marker PLFAs (Table 4; see Table S3 for atom\% values).

DFA suggested strong similarity in the composition of PLFAs in BB and BA rhizotrons. Both treatments differed strongly from AA and control treatments (Fig. 2). Differences were mainly due to low amounts of gram-negative (cy17:0) and gram-positive bacteria (i17:0) in beech rhizotrons. Higher fungal biomass and low $\mathrm{pH}$ in bee ch and mixed rhizotrons also contributed to the separation of these treatments but to a lower extent (Tables 5,6). Pyrosequencing of the bacterial community revealed high overlap of bacterial phyla and species with little differences between the treatments (Fig. 3).

\section{Soil fauna / gamasid mites}

The $\delta^{13} \mathrm{C}$ and $\delta^{15} \mathrm{~N}$ from the added ash litter was incorporated into basal species of the soil food web as indicated by the label in the predatory mite H. aculeifer (Table 4; see Table S3 for atom\% values). The signatures suggest that incorporation of label was most pronounced in mixed rhizotrons (significant interaction between beech and ash) followed by $A A, B B$ and control rhizotrons. Abundances of soil animal taxa extracted by heat, i.e., collembolans, gamasid and oribatid mites as well as earthworms, generally did not differ between treatments (Table S4). 


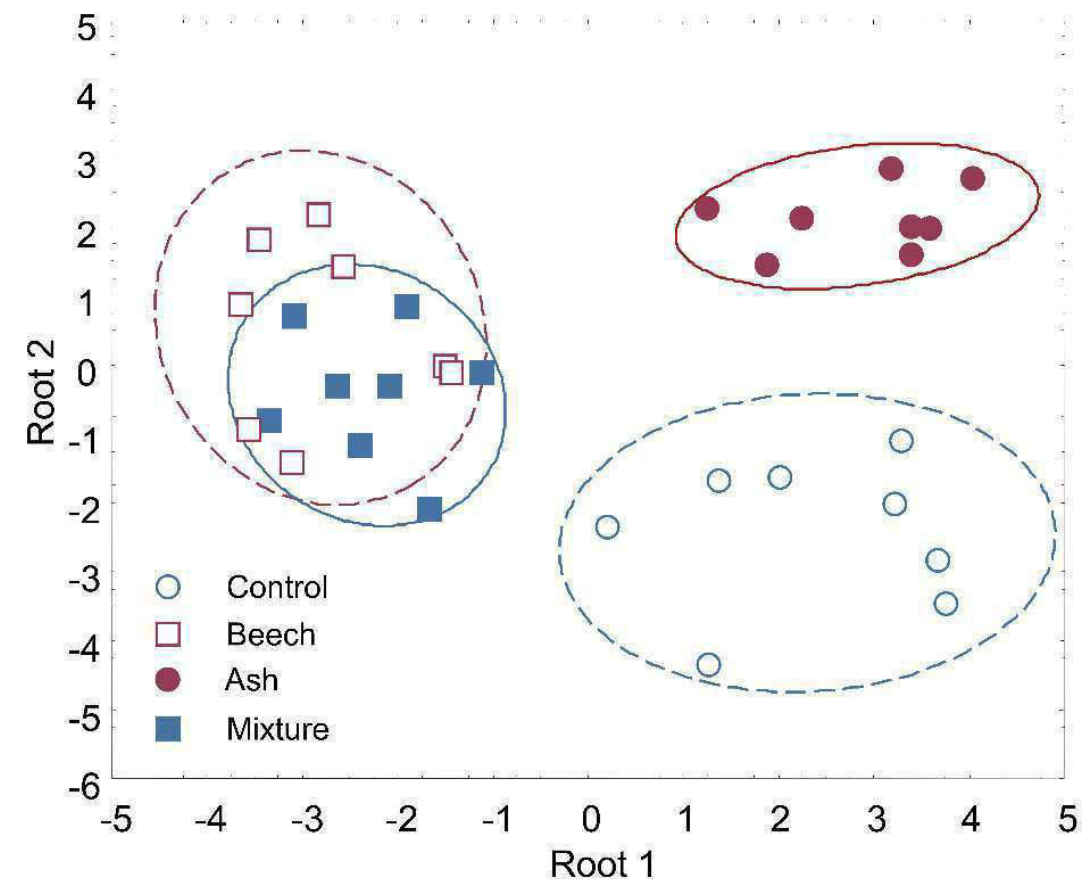

Figure 4 | Discriminant function analysis (DFA) of microbial P LFAs, microbial respiration and soil properties in rhizotrons without trees (control), with beech, ash and a mixture of beech and ash. Wilks' Lambda $0.0165, F_{(54,33)}$ $=1.85, P=0.0296$. Ellipses represent confidence intervals at $P=0.05$.

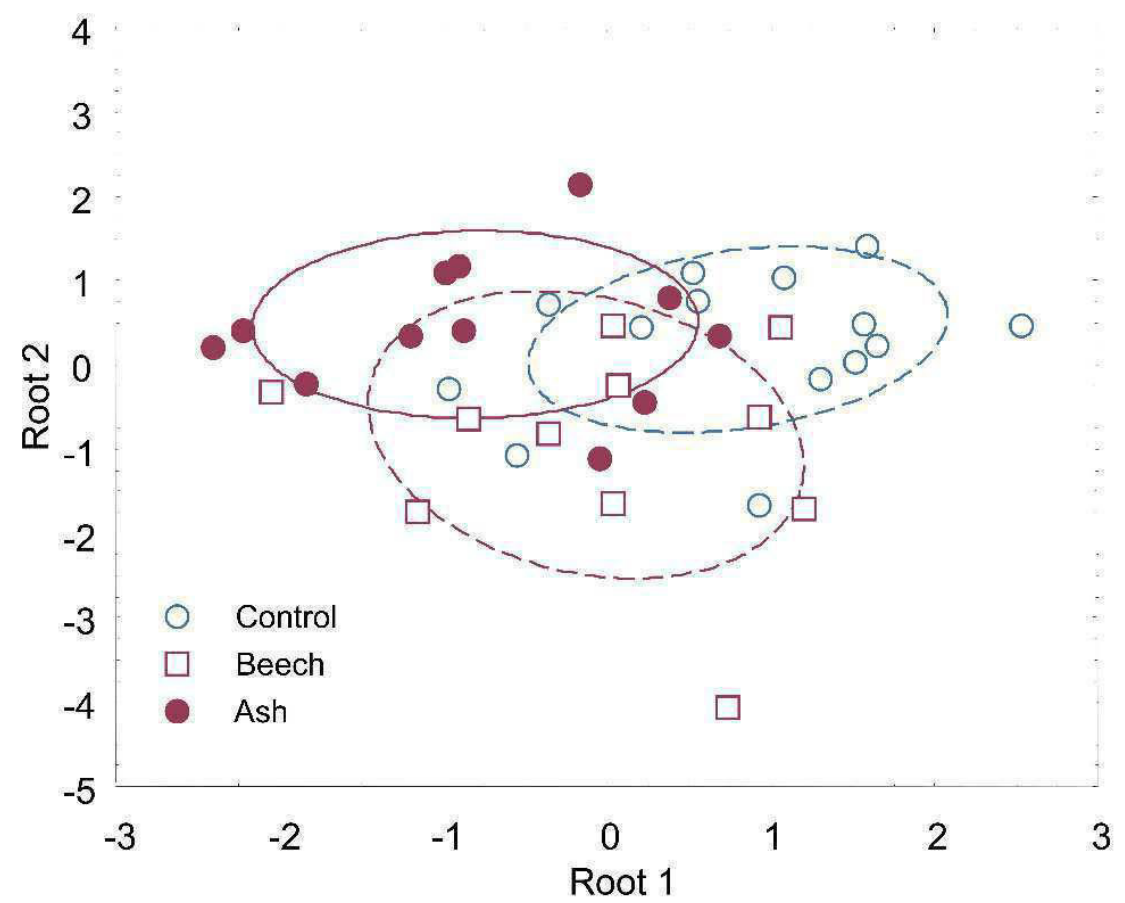

Figure 5 | Discriminat function analysis (DFA) of bacterial ph yla based on pyrosequencing of 16S rRNA in rhizotrons without trees (control) and with beech and ash seedlings after reducing data to six dimensions by non-multidim ensional scaling (NMDS). Wilks' Lambda 0.4996; $F_{(12,60)}=2.07 ; P=0.0325$. Ellipses represent confidence intervals at $P=0.05$. 


\section{Discussion}

\section{Changes in the microbial community due to rhizodeposition}

Lower $\mathrm{pH}$ in the rhizosphere of beech likely contributed to favoring soil fungi supporting our hypothesis (1) that beech and ash differentially affect the structure of the microbial community. Acidification of the soil by beech is well known (Holzwarth et al., 2011; Langenbruch et al., 2012), however, commonly it has been ascribed to low concentrations of calcium and magnesium and high concentrations of recalcitrant compounds such as lignin in beech leaf litter (Reich et al., 2005; Hobbie et al., 2006; Hansen et al., 2009). As we excluded leaf litter fall from seedlings to the rhizotron so il surface and uniformly placed high quality ash litter in each of the treatments, the observed differences must have been due to the activity of beech roots. Indeed, in the vicinity of beech roots concentrations of formate and acetate were increased as compared to control rhizotrons in the same experiment, whereas in the vicinity of ash roots only the concentration of ace tate increased (Fender et al., 2013). The release of organic acids increases nutrient availability and this is facilitated by low pH (Jones et al., 2004); presumably, beech employs this strategy to increase nutrient mobilization and uptake. Low pH in the soil, however, predominantly is caused by the release of $\mathrm{H}^{+}$by roots rather than by dissociation of organic acids (Neumann and Römheld, 1999). Notably, acidification of the soil by beech roots occurred despite a comparatively lower root biomass in beech than ash rhizotrons. However, SRA and SRL were higher in B+ rhizotrons as compared to $A+$ rhizotrons. This suggests that the observed modifications were partly due to changes in root physiology rather than root biomass and number of fine root tips (Lehmann, 2003). Differences in the release rates of specific exudates of the two species presumably also contributed to the observed changes. 
Bacterial community composition was little affected by tree roots as indicated by analysis of 16S rRNA. The ratio of fungal-to-bacterial biomass measured via fatty acid analysis increased in $\mathrm{B}+$ rhizotrons and reflected the general pattern of increasing fungal dominance at low $\mathrm{pH}$ accounting for differences in soil processes (Aciego Pietri and Brookes, 2008; Rousk et al., 2009). Fungal biomass was measured using $18: 2 \omega 6,9$ as marker PLFA (Ruess and Chamberlain, 2010; Frostegård et al., 2011) which includes EM and saprotrophic fungi (Kaiser, Frank, et al., 2010). We suggest the change in fungal biomass to refer not to AM fungi since the PLFA $18: 2 \omega 6,9$ is only found in very low densities in this type of fungi (Olsson and Johansen, 2000) and since the AM colonization rate did not change. Colonization by EM fungi in beech was relatively low ( $46 \pm 6 \%$, pooled data from $\mathrm{BB}$ and $\mathrm{BA}$ rhizotrons). This corresponds to low colonization rates in other greenhouse and rhizotron experiments (Dučić et al., 2009; Reich et al., 2009; Winkler et al., 2010) when compared to field data (Leuschner et al., 2004; Lang et al., 2011). Low EMF colonization rate and a stronge $r$ depletion of $\delta^{13} C$ of PLFA $18: 2 \omega 6,9$ in $B+$ rhizotrons point to SOM decomposition suggesting th at saprotrophic rather than EM fungi increased in beech rhizotrons as fine root tips and mycorrhiza were shown to have relatively similar signatures, whereas soil is stronger depleted in $\delta^{13} \mathrm{C}$ (Eissfeller et al., 2013). We therefore suggest saprotrophic fungi to substantially contribute to changes in the fungal PLFA marker.

Combined data on PLFAs, soil properties and microbi al respiration revealed high similarity of beech and mixed rhizotrons in DFA with these differing significantly from ash and control rhizotrons. The fatty acids $i 17: 0$ and cy17:0 contributed most to this separation, with lesser contribution by $\mathrm{pH}$ and fungal biomass. The fatty ac id i17:0 is regarded as marker for grampositive bacteria whereas cy 17:0 characterizes gram-negative bacteria, the former considered to dominate in microorganisms being present in bulk soil whereas the latter in rhizosphere 
soil processing labile root derived carbon (Söderberg et al., 2004; Paterson et al., 2007). The relative abundance of both was lowest in BB rhizotrons suggesting that both suffered from the presence of beech roots, presumably due to beech increasing the competitive strength of saprotrophic fungi.

\section{Changes in decomposition due to different tree species}

Hypothesis (2) assuming that litter decomposition is differentially affected by tree species was supported by our data. Generally, stable isotope values of the litter-soil mixture in ES decreased strongly during incubation. Ash litter is known to decompose fast; in the field it disappears entirely after two years (Jacob et al., 2009). High and constant temperatures within the climate chambers $\left(20^{\circ} \mathrm{C}\right)$ contributed to fast decomposition of the litter in the rhizotrons. Data on higher $\mathrm{qO}_{2}$ (this study) and higher cumulative heterotrophic $\mathrm{CO}_{2}$ production in beech as compared to ash rhizotrons (Fender et al., 2013) suggest an overall higher stimulation of litter decomposition in beech root affected soil, i.e., higher carbon loss due to microbial respiration. High $\mathrm{H}^{+}$concentrations have been shown to limit bacterial growth, while low concentrations limit fungal growth (Rousk et al., 2009). The fact that $q \mathrm{O}_{2}$ increased whereas bacterial biomass did not change suggests that the metabolic costs of rhizosphere bacteria increased at least at the end of the experiment. Presumably, lower soil pH in beech rhizotrons decreased the efficiency of bacteria to use carbon for biomass production by increasing respiratory losses.

$\delta^{13} \mathrm{C}$ values in fungal and bacterial PLFAs were depleted most in $\mathrm{B}+$ rhizotrons suggesting that bacteria and fungi incorporated less litter carbon in presence of beech roots than of ash also indicating a faster turnover of litter carbon. Further, the more depleted $\delta^{13} \mathrm{C}$ values in fungi compared to beech fine roots suggest that fungal carbon originated from soil organic matter 
in beech rhizotrons, whereas higher $\delta^{13} \mathrm{C}$ values in bacteria rather suggest bacteria to depend on root-derived carbon as their signatures resembled that of beech fine roots (Bowling et al., 2008).

Several studies found plant species identity to have stronger effects than plant diversity (De Deyn et al., 2004; Hättenschwiler and Gasser, 2005; Ball et al., 2009), with certain plant species acting as key species (Jacob et al., 2009). The strong effect of beech in this study is mediated by roots whereas ash had no effect suggesting that rhizodeposition in ash is of minor importance. Despite this low rhizosphere changes ash incorporated more litter nitrogen than beech (Lang and Polle, 2011; Schulz et al., 2011); potentially, ash is more effective in exploiting resources from fast decomposing litter such as ash leaves or by virtue of the higher root biomass production of ash in our experiment. Notably, ash seedlings incorporated more litter

${ }^{15} \mathrm{~N}$ than beech seedlings supporting the conclusion that the reduced $\mathrm{N}_{\text {total }}$ in $\mathrm{B}+$ rhizotrons was due to increased SOM decomposition and not due to plant uptake by beech. Notably, the uptake of ${ }^{15} \mathrm{~N}$ declined in mixture with ash. This corresponds to field observations where the $\mathrm{N}$ concentrations in ash declined in mixtures with other tree species and their ectomycrrhizal diversity (Lang and Polle, 2011). A higher uptake of $\mathrm{N}$ by ash roots was also found in a ${ }^{15} \mathrm{~N}$ tracer study in the Hainich forest where ash fine roots showed a significantly higher massspecific uptake of labeled $\mathrm{NH}_{4}^{+}$and glycine (but not of $\mathrm{NO}_{3}{ }^{-}$) than beech roots (A. Jacob, unpubl.results).

\section{Channeling of litter-derived carbon into higher trophic levels}

Hypothesis (3) assuming that mixing of both tree species beneficially affects microorganisms thereby stimulating carbon turnover is supported in part by our data. Generally, mixing of tree species increased plant biomass, fine root tips, SRA, SRL and mycorrhizal colonization especially that of beech seedlings but did not affect soil chemistry and microbial biomass. 
However, soil chemistry and microbial data are point measures and do not reflect fluxes over the whole period of the experiment. As the plants are sinks for resources made available over the whole experimental time higher plant growth in mixed rhizotrons suggests that the gross flux of resources was greater in mixed rhizotrons.

Isotope analyses of food web components are a net measure over the long experimental period. Here, we measured $\delta^{13} \mathrm{C}$ and $\delta^{15} \mathrm{~N}$ being incorporated within the predatory mite $H$. aculeifer. $\delta^{13} \mathrm{C}$ and $\delta^{15} \mathrm{~N}$ values of $H$. aculeifer were significantly increased in mixed and ash rhizotrons suggesting that more litter-derived carbon and nitrogen entered basal species of the soil food web which served as prey for gamasid mites, such as nematodes and collembolans feeding on bacteria and fungi. In cont rast, in control and beech rhizotrons $\delta^{13} \mathrm{C}$ values of $H$. aculeifer resembled those in Hainich beech forests $\left(\delta^{13} \mathrm{C}:-23.9 \pm 0.76 \%\right.$; $\delta^{15} \mathrm{~N}$ : $+2.0 \pm 2.11 \%$; Klarner et al., 2013) suggesting low incorporation of litter-derived carbon (and nitrogen) into the prey of $H$. aculeifer. However, the turnover of belowground $\mathrm{C}$ in unplanted soil, i.e., the control, was numerously shown to be lower compared to planted soil (Kuzyakov, 2010; Bird et al., 2011), i.e., soil with beech trees. Low incorporation of litter resources in BB rhizotrons may point to the fast decomposition of a sh litter and to the dominance of root derived resources as basis of the soil animal food web in beech forests as suggested earlier (Pollierer et al., 2007). Of course, measurements of a single species, i.e., H. aculeifer, do not allow to predict carbon and nitrogen cycling through the whole soil food web. However, since the soil fauna composition within the rhizotrons did not differ, we suggest tree species to significantly affect the amount and the way carbon is channeled through the soil food web. 


\section{Conclusions}

The results suggest that the effect of living roots on litter decomposition, SOM dynamics and energy channels varies with tree species identity. Rhizodeposits have the potential to change soil $\mathrm{pH}$ with the potential to affect the metabolic activity of microorganisms. This propagates to higher trophic levels as tree species can impact the amount of litter-derived resource entering the soil food web and on energy channels. Effects of living roots are notoriously understudied and have to be included into studies on soil $\mathrm{C}$ dynamics to understand carbon and nutrient cycling as well as soil food web funct ioning of forests.

\section{Acknowledgements}

We thank Dr. Lars Köhler and Dr. Heinz Coners for technical support and Martin Blumberg for GC-MS measurements. This study was funded by the Ministry of Science and Culture of Lower Saxony and the 'Niedersächsisches Vorab' as part of the Cluster of Excellence 'Functional Biodiversity Research'.

\section{References}

Aciego Pietri, J.C., Brookes, P.C., 2008. Relationships between soil pH and microbial properties in a UK arable soil. Soil Biology and Biochemistry 40, 1856-1861.

Anderson, J.P.E., Domsch, K.H., 1978. A physiological method for the quantitative measurement of microbial biomass in soils. Soil Bio logy and Biochemistry 10, 215-221.

Ball, B.A., Bradford, M.A., Coleman, D.C., Hunter, M.D., 2009. Linkages between below and aboveground communities: Decomposer responses to simulated tree species loss are largely additive. Soil Biology and Biochemistry 41, 1155-1163. 
Beck, T., Joergensen, R.G., Kandeler, E., Makeschin, E., Nuss, E., Oberholzer, H.R., Scheu, S., 1997. An inter-laboratory comparison of ten different ways of measuring soil microbial biomass C. Soil Biology and Biochemistry 29, 1023-1032.

Bird, J.A., Herman, D.J., Firestone, M.K., 2011. Rhizosphere priming of soil organic matter by bacterial groups in a grassland soil. Soil Biology and Biochemistry 43, 718-725.

Bligh, E.G., Dyer, W.J., 1959. A rapid method of total lipid extraction and purification. Journal of Biochemistry and Physiology 37, 911-917.

Bowling, D.R., Pataki, D.E., Randerson, J.T., 2008. Carbon isotopes in terrestrial ecosystem pools and $\mathrm{CO}_{2}$ fluxes. New Phytologist 178, 24-40.

Broadmeadow, M., Duncan, R., 2005. Climate Change and British Woodland. Forestry Commission Information Note 69.

Butler, J.L., Bottomley, P.J., Griffith, S.M., Myrold, D.D., 2004. Distribution and turnover of recently fixed photosynthate in ryegrass rhizospheres. Soil Biology and Biochemistry 36, 371-382.

De Deyn, G.B., Raaijmakers, C.E., Ruijven, J.V., Berendse, F., van der Putten, W.H., 2004. Plant species identity and diversity effects on different trophic levels of nematodes in the soil food web. Oikos 106, 576-586.

Dučić, T., Berthold, D., Langenfeld-Heyser, R., Beese, F., Polle, A., 2009. Mycorrhizal communities in relation to biomass production and $\mathrm{n}$ utrient use efficiency in two varieties of Douglas fir (Pseudotsuga menziesii var. menziesii and var. glauca) in different forest soils. Soil Biology and Biochemist ry 41, 742-753.

Edgar, R.C., 2010. Search and clustering orders of magnitude faster than BLAST. Bioinformatics 26, 2460-2461.

Eissfeller, V., Beyer, F., Valtanen, K., Hertel, D., Maraun, M., Polle, A., Scheu, S., 2013. Incorporation of plant carbon and microbial nitroge $n$ into the rhizosphere food web of beech and ash. Soil Biology and Biochemistry, in press.

Emborg, J., 1998. Understorey light conditions and regeneration with respect to the structural dynamics of a near-natural temperate deciduous forest in Denmark. Forest Ecology and Management 106, 83-95.

Fender, A.-C., Gansert, D., Jungkunst, H.F., Fiedler, S., Beyer, F., Schützenmeister, K., Thiele B., Valtanen, K., Polle, A., Leschner, C., (2013). Root-induced tree species effects on the 
source/sink strength for greenhouse gases $\left(\mathrm{CH}_{4}, \mathrm{~N}_{2} \mathrm{O}\right.$ and $\left.\mathrm{CO}_{2}\right)$ of a temperate deciduous forest soil, Soil Biology and Biochemistry 57, 587-597.

Frostegård, Å., Tunlid, A., Bååth, E., 1991. Microbial biomass measured as total lipid phosphate in soils of different organic content. Jo urnal of Microbiological Methods 4, 151-163.

Frostegård, Å., Tunlid, A., Bååth, E., 2011. Use and misuse of PLFA measurements in soils. Soil Biology and Biochemistry 43, 1621-1625.

Gartner, T.B., Cardon, Z.G., 2004. Decomposition dynamics in mixed-species leaf litter. Oikos 104, 230-246.

Gessner, M.O., Swan, C.M., Dang, C.K., McKie, B.G., Bardgett, R.D., Wall, D.H., Hättenschwiler, S., 2010. Diversity meets decomposition. Trends in Ecology \& Evolution 25, 372-380.

Grayston, S.J., Wang, S., Campbell, C.D., Edwards, A.C., 1998. Selective influence of plant species on microbial diversity in the rhizosphere. Science 30, 369-378.

Gregory, P.J., 2006. Roots, rhizosphere and soil: the route to a better understanding of soil science? European Journal of Soil Science 57, 2-12.

Grime, J.P., Thompson, K., Hunt, R., Hodgson, J.G., Cornelissen, J.H.C., Rorison, I.H., Hendry, G.A.F., Ashenden, T.W., Askew, A.P., Band, S.R., Booth, R.E., Bossard, C.C., Campbell, B.D., Cooper, J.E.L., Davison, A.W., Gupta, P.L., Hall, W., Hand, D.W., Hannah, M.A., Hillier, S.H., Hodkinson, D.J., Jalili, A., Liu, Z., Mackey, J.M.L., Matthews, N., Mowforth, M.A., Neal, A.M., Reader, R.J., Reiling, K., Ross-Fraser, W., Spencer, R.E., Sutton, F., Tasker, D.E., Thorpe, P.C., Whitehouse, J., 1997. Integrated screening validates primary axes of specialisation in plants. Oikos 79, 259-281.

Hansen, K., Vesterdal, L., Schmidt, I.K., Gundersen, P., Sevel, L., Bastrup-Birk, A., Pedersen, L.B., Bille-Hansen, J., 2009. Litterfall and nutrient return in five tree species in a common garden experiment. Forest Ecology and Management 257, 2133-2144.

Hobbie, S.E., Reich, P.B., Oleksyn, J., Ogdahl, M., Zytkowiak, R., Hale, C., Karolewski, P., 2006. Tree species effects on decomposition and forest floor dynamics in a common garden. Ecology 87, 2288-2297.

Holzwarth, F.M., Daenner, M., Flessa, H., 2011. Effects of beech and ash on small-scale variation of soil acidity and nutrient stocks in a mixed deciduous forest. Journal of Plant Nutrition and Soil Science 174, 799-808. 
Hättenschwiler, S., Gasser, P., 2005. Soil animals alter plant litter diversity effects on decomposition. Proceedings of the National Academy of Sciences of the United States of America 102, 1519-1524.

Hättenschwiler, S., Tiunov, A.V., Scheu, S., 2005. Biodiversity and Litter Decomposition in Terrestrial Ecosystems. Annual Review of Ecology, Evolution, and Systematics 36, 191 218.

Jacob, M., Viedenz, K., Polle, A., Thomas, F.M., 2010. Leaf litter decomposition in temperate deciduous forest stands with a decreasing fraction of beech (Fagus sylvatica). Oecologia 164, 1083-1094.

Jacob, M., Weland, N., Platner, C., Schaefer, M., Leuschner, C., Thomas, F.M., 2009. Nutrient release from decomposing leaf litter of temperate deciduous forest trees along a gradient of increasing tree species diversity. Soil Biology and Biochemistry 41, 21222130.

Jones, D.L., Hodge, A., Kuzyakov, Y., 2004. Plant and mycorrhizal regulation of rhizodeposition. New Phytologist 163, 459-480.

Kaiser, C., Frank, A., Wild, B., Koranda, M., Richter, A., 2010. Negligible contribution from roots to soil-borne phospholipid fatty acid fungal biomarkers 18:2w6,9 and 18:1w9. Soil Biology and Biochemistry 42, 1650-1652.

Kempson, D., Lloyd, M., Ghelardi, R., 1963. A new extractor for woodland litter. Pedobiologia 3, 1-21.

Klarner, B., Maraun, M., Scheu, S., 2012. Trophic diversity and niche partitioning in a species rich predator guild - Natural variations in stable isotope ratios $\left({ }^{13} \mathrm{C} /{ }^{12} \mathrm{C},{ }^{15} \mathrm{~N} /{ }^{14} \mathrm{~N}\right)$ of mesostigmatid mites (Acari, Mesostigmata) from Central European beech forests. Soil Biology and Biochemistry, doi.org/10.1016/j.soilbio .2012.08.013.

Kuzyakov, Y., 2010. Priming effects: Interactions between living and dead organic matter. Soil Biology and Biochemistry 42, 1363-1371.

Lang, C., Seven, J., Polle, A., 2011. Host preferences and differential contributions of deciduous tree species shape mycorrhizal species richness in a mixed Central European forest. Mycorrhiza 21, 297-308.

Lang, C., Polle, A., 2011, Ectomycorrhizal fungal diversity, tree diversity and root nutrient relations in a mixed Central European forest. Tree Physiology 31, 531-538. 
Langenbruch, C., Helfrich, M., Flessa, H., 2012. Effects of beech (Fagus sylvatica), ash (Fraxinus excelsior) and lime (Tilia spec.) on soil chemical properties in a mixed deciduous forest. Plant and Soil 352, 389-403.

Lehmann, J., 2003. Subsoil root activity in tree-based cropping systems. Plant and Soil 255, 319-331.

Leuschner, C., Hertel, D., Schmid, I., Koch, O., Muhs, A., Hölscher, D., 2004. Stand fine root biomass and fine root morphology in old-growth beech forests as a function of precipitation and soil fertility. Plant and Soil 25 8, 43-56.

McGonigle, T.P., Millers, M.H., Evans, D.G., Fairchild, G.L., Swan, J.A., 1990. A new method which gives an objective measure of colonization of roots by vesicular-arbuscular mycorrhizal fungi. New Phytologist 115, 495-501.

McKinley, D.C., Ryan, M.G., Birdsey, R.A., Giardina, C.P., Harmon, M.E., Heath, L.S., Houghton, R.A., Jackson, R.B., Morrison, J.F., Murray, B.C., Pataki, D.E., Skog, K.E., 2011. A synthesis of current knowledge on forests and carbon storage in the United States. Ecological Applications 21, 1902-1924.

Meinen, C., Hertel, D., Leuschner, C., 2009. Biomass and morphology of fine roots in temperate broad-leaved forests differing in tree species diversity: is there evidence of below-ground overyielding? Oecologia 161, 99-111.

Moore-Kucera, J., Dick, R.P., 2008. Application of 13C-labeled litter and root materials for in situ decomposition studies using phospholipid fatty acids. Soil Biology and Biochemistry 40, 2485-2493.

Nacke, H., Thürmer, A., Wollherr, A., Will, C., Hodac, L., Herold, N., Schöning, I., Schrumpf, M., Daniel, R., 2011. Pyrosequencing-based assessment of bacterial community structure along different management types in German forest and grassland soils. PloS one 6, e17000.

Neumann, G., Römheld, V., 1999. Root excretion of carboxylic acids and protons in phosphorus-deficient plants. Plant and Soil 211, $121-130$.

Olsson, P.A., Johansen, A., 2000. Lipid and fatty a cid composition of hyphae and spores of arbuscular mycorrhizal fungi at different growth stages. Mycological Research 104, 429434.

Paterson, E., Gebbing, T., Abel, C., Sim, A., Telfer, G., 2007. Rhizodeposition shapes rhizosphere microbial community structure in organic soil. New Phytologist 173,600 610. 
Paterson, E., Midwood, A.J., Millard, P., 2009. Through the eye of the needle: a review of isotope approaches to quantify microbial processes mediating soil carbon balance. New Phytologist 184, 19-33.

Pollierer, M.M., Langel, R., Körner, C., Maraun, M., Scheu, S., 2007. The underestimated importance of belowground carbon input for forest soil animal food webs. Ecology Letters 10, 729-736.

Reeder, J., Knight, R., 2010. Rapidly denoising pyrosequencing amplicon reads by exploiting rank abundance distributions. Nature Methods 7, 668-669.

Reich, M., Göbel, C., Kohler, A., Buée, M., Martin, F., Feussner, I., Polle, A., 2009. Fatty acid metabolism in the ectomycorrhizal fungus Laccaria bicolor. New Phytologist 182, $950-$ 964.

Reich, P.B., Oleksyn, J., Modrzynski, J., Mrozinski, P., Hobbie, S.E., Eissenstat, D.M., Chorover, J., Chadwick, O.A., Hale, C.M., Tjoelker, M.G., 2005. Linking litter calcium, earthworms and soil properties: a common garden test with 14 tree species. Ecology Letters 8, 811818.

Rousk, J., Brookes, P.C., Bååth, E., 2009. Contrasting soil pH effects on fungal and bacterial growth suggest functional redundancy in carbon mine ralization. Applied and Environmental Microbiology 75, 1589-1596.

Ruess, L., Chamberlain, P.M., 2010. The fat that matters: Soil food web analysis using fatty acids and their carbon stable isotope signature. So il Biology and Biochemistry 42, 18981910.

Scheu, S., 1992. Automated measurement of the respiratory response of soil microcompartments: active microbial biomass in earthworm faces. Soil Biology and Biochemistry 24, 1113-1118.

Schlesinger, W.H., Andrews, J.A., 2000. Soil respiration and the global carbon cycle. Biogeochemistry 48, 7-20.

Schloss, P.D., Handelsman, J., 2005. Introducing DOTUR, a computer program for defining operational taxonomic units and estimating species richness. Applied and Environmental Microbiology 71, 1501-1506.

Schmalenberger, A., Schwieger, F., Tebbe, C., 2001. Effect of primers hybridizing to different evolutionarily conserved regions of the small-subun it rRNA Gene in PCR-based microbial community analyses and genetic profiling. Applied and Environmental Microbiology 67, 3557-3563. 
Schmitz, O., Danneberg, G., Hundeshagen, B., Klinger, A., Bothe, H., 1991. Quantification of vesicular-arbuscular mycorrhiza by biochemical parameters. Journal of Plant Physiology $139,104-114$.

Schulz, H., Härtling, S., Stange, C.F., 2011. Species-specific differences in nitrogen uptake and utilization by six European tree species. Journal of Plant Nutrition and Soil Science 174, 28-37.

Smith, S., Read, D.J., 2008. Mycorrhizal symbiosis. Cambridge, UK, Academic Press.

Steinbeiss, S., Beßler, H., Engels, C., Temperton, V.M., Buchmann, N., Roscher, C., Kreutziger, Y., Baade, J., Habekost, M., Gleixner, G., 2008. Plant diversity positively affects shortterm soil carbon storage in experimental grasslands. Global Change Biology 14, 29372949.

Swift, M.J., Heal, O.W., Anderson, J.M., 1979. Decomposition in Terrestrial Ecosystems. University of California Press, Berkeley.

Söderberg, K.H., Probanza, A., Jumpponen, A., Bååth, E., 2004. The microbial community in the rhizosphere determined by community-level physiological profiles (CLPP) and direct soil- and cfu-PLFA techniques. Applied Soil Ecology 25, 135-145.

Wardle, D.A., 1998. Review controls of temporal variability of the soil microbial biomass: a global-scale synthesis. Soil Biology and Biochemist ry 30, 1867-1878.

Weber-Blaschke, G., Claus, M., Rehfuss, K.E., 2002. Growth and nutrition of ash (Fraxinus excelsior L.) and sycamore (Acer pseudoplatanus L.) on soils of different base saturation in pot experiments. Forest Ecology and Management 167, 43-56.

Winkler, J.B., Dannenmann, M., Simon, J., Pena, R., Offermann, C., Sternad, W., Clemenz, C., Naumann, P.S., Gasche, R., Kögel-Knabner, I., Gessler, A., Rennenberg, H., Polle, A, 2010. Carbon and nitrogen balance in beech roots under competitive pressure of soilborne microorganisms induced by girdling, drought and glucose application. Functional Plant Biology 37, 879-889. 
Table S1 | Atom\% values of the used soil, labeled ash litter and of the soil-litter mixture in experimental sites at the start of the experiment and at the end after 422 days of litter incubation (means $\pm 1 \mathrm{SE}$ ). Soil, litter and the soil-litter mixture samples at the start were replicated $\mathrm{n}=5$, whereas soil-litter mixture samples of the end were pooled across all treatments $(n=16)$.

\begin{tabular}{|c|c|c|c|c|c|c|c|c|}
\hline & \multicolumn{6}{|c|}{ Start } & \multirow{2}{*}{\multicolumn{2}{|c|}{$\begin{array}{c}\text { End } \\
\text { Soil-litter mixture } \\
\end{array}$}} \\
\hline & \multicolumn{2}{|c|}{ Soil } & \multicolumn{2}{|c|}{ Litter } & \multicolumn{2}{|c|}{ Soil-litter mixture } & & \\
\hline & Mean & SE & Mean & SE & Mean & SE & Mean & SE \\
\hline atom $\%{ }^{13} \mathrm{C}$ & 1.0769728 & \pm 0.00009197 & $1.2659526=$ & \pm 0.0003437 & 1.1810216 & \pm 0.00070136 & 1.08698261 & \pm 0.0020640 \\
\hline atom $\%{ }^{15} \mathrm{~N}$ & 0.36690135 & \pm 0.00006571 & $4.941404787=$ & \pm 0.01963241 & 2.56267058 & \pm 0.02469798 & 0.57583439 & \pm 0.04140770 \\
\hline
\end{tabular}


Table S2 | Means \pm 1 SE of atom\% values of plant parameters influenced by beech (B) and ash (A) in rhizotrons after 475 days $(n=4)$.

\begin{tabular}{|c|c|c|c|c|c|c|c|c|c|c|c|c|}
\hline \multirow[b]{3}{*}{ atom $\%{ }^{13} \mathrm{C}$} & \multicolumn{3}{|c|}{$\begin{array}{c}\text { BB } \\
\text { (pure beech) } \\
\end{array}$} & \multicolumn{3}{|c|}{$\begin{array}{c}\text { AA } \\
\text { (pure ash) }\end{array}$} & \multicolumn{3}{|c|}{$\begin{array}{c}\text { BA } \\
\text { (beech in mixture) }\end{array}$} & \multicolumn{3}{|c|}{$\begin{array}{c}\mathbf{A B} \\
\text { (ash in mixture) }\end{array}$} \\
\hline & \multirow[t]{2}{*}{ Means } & \multirow{2}{*}{\multicolumn{2}{|c|}{ SE }} & \multirow[t]{2}{*}{ Means } & \multirow{2}{*}{\multicolumn{2}{|c|}{ SE }} & \multirow[t]{2}{*}{ Means } & \multirow{2}{*}{\multicolumn{2}{|c|}{ SE }} & \multirow[t]{2}{*}{ Means } & & \multirow[t]{2}{*}{ SE } \\
\hline & & & & & & & & & & & & \\
\hline Shoot & 1.07383513 & \pm & 0.00017420 & 1.07495600 & \pm & 0.00015455 & 1.07514475 & \pm & 0.00017318 & 1.07568600 & \pm & 0.00019963 \\
\hline Leave & 1.07326575 & \pm & 0.00030884 & 1.07365156 & \pm & 0.00015001 & 1.07362325 & \pm & 0.00034224 & 1.07303038 & \pm & 0.00015562 \\
\hline Fine roots & 1.07465313 & \pm & 0.00018487 & 1.07750778 & \pm & 0.00046296 & 1.07596411 & \pm & 0.00014480 & 1.07730790 & \pm & 0.00017922 \\
\hline Coarse roots & 1.07542997 & \pm & 0.00016796 & 1.07765847 & \pm & 0.00041694 & 1.07558569 & \pm & 0.00023790 & 1.07770568 & \pm & 0.00024885 \\
\hline \multicolumn{13}{|l|}{ atom $\%{ }^{15} \mathrm{~N}$} \\
\hline Shoot & 0.42876468 & \pm & 0.00559039 & 0.46109969 & \pm & 0.01205151 & 0.42266801 & \pm & 0.00472628 & 0.42261393 & \pm & 0.00689963 \\
\hline Leave & 0.43647468 & \pm & 0.00595216 & 0.48166205 & \pm & 0.00789685 & 0.42708961 & \pm & 0.00605381 & 0.44954088 & \pm & 0.00388855 \\
\hline Fine roots & 0.43692704 & \pm & 0.00760428 & 0.50868574 & \pm & 0.01805715 & 0.43140529 & \pm & 0.00454258 & 0.46031359 & \pm & 0.00587501 \\
\hline Coarse roots & 0.44251012 & \pm & 0.00506091 & 0.51054955 & \pm & 0.01435028 & 0.42058708 & \pm & 0.00324815 & 0.44450474 & \pm & 0.00495442 \\
\hline
\end{tabular}


Table S3 | Means \pm 1 SE of atom\% values of soil C and N, PLFA and gamasid mites as influenced by beech (B) and ash (A) in rhizotrons after 475 days ( $n=4)$.

\begin{tabular}{|c|c|c|c|c|c|c|c|c|c|}
\hline \multirow[b]{4}{*}{ soil data } & \multicolumn{4}{|c|}{ B- } & \multicolumn{5}{|c|}{ B+ } \\
\hline & \multicolumn{2}{|c|}{$\begin{array}{c}\text { A- } \\
\text { (Control) }\end{array}$} & \multicolumn{2}{|c|}{$\begin{array}{c}\text { A+ } \\
\text { (Ash) }\end{array}$} & \multicolumn{2}{|c|}{$\begin{array}{c}\text { A- } \\
\text { (Beech) }\end{array}$} & \multicolumn{3}{|c|}{$\begin{array}{c}\text { A+ } \\
\text { (Mixture) }\end{array}$} \\
\hline & Mean & SE & Mean & SE & Mean & SE & Mean & & SE \\
\hline & \multirow[b]{2}{*}{1.08291691} & \multirow[b]{2}{*}{0.00068504} & \multirow[b]{2}{*}{1.08691934} & \multirow[b]{2}{*}{0.00267118} & \multirow[b]{2}{*}{1.08061441} & \multirow[b]{2}{*}{0.00267118} & \multirow[b]{2}{*}{1.07997306} & \multirow[b]{2}{*}{ \pm} & \multirow[b]{2}{*}{0.00267118} \\
\hline atom $\%{ }^{13} \mathrm{C}$ & & & & & & & & & \\
\hline atom $\%{ }^{15} \mathrm{~N}$ & 0.4995646 & \pm 0.02435464 & 0.57079355 & \pm 0.05107642 & 0.44495352 & \pm 0.05107642 & 0.43470143 & \pm & 0.05107642 \\
\hline \multicolumn{10}{|l|}{ PLFA } \\
\hline Total (atom $\%{ }^{13} \mathrm{C}$ ) & 1.08072624 & \pm 0.00041531 & 1.08215226 & \pm 0.00067208 & 1.07597482 & \pm 0.00038549 & 1.07989923 & \pm & 0.00226927 \\
\hline Bacteria (atom $\%{ }^{13} \mathrm{C}$ ) & 1.07898895 & \pm 0.00120338 & 1.07894481 & \pm 0.00082096 & 1.07585318 & \pm 0.00033924 & 1.07797283 & \pm & 0.00043242 \\
\hline Fungi (atom $\%{ }^{13} \mathrm{C}$ ) & 1.08268228 & \pm 0.00023938 & 1.08700051 & \pm 0.00061554 & 1.07110968 & \pm 0.00036185 & 1.07473461 & \pm & 0.00054633 \\
\hline \multicolumn{10}{|l|}{ gamasid mites } \\
\hline atom $\%{ }^{13} \mathrm{C}$ & 1.08010350 & \pm 0.00093649 & 1.09026840 & \pm 0.00034029 & 1.08358067 & \pm 0.00152939 & 1.09606000 & \pm & 0.00046706 \\
\hline atom $\%{ }^{15} \mathrm{~N}$ & 0.41377425 & \pm 0.00841666 & 0.62595061 & \pm 0.01575008 & 0.48989230 & \pm 0.01359816 & 0.77384461 & \pm & 0.00976060 \\
\hline
\end{tabular}


Table S4 | ANOVA table of $F$ - and $P$-values as well as means \pm 1 SE for soil animal taxa extracted by heat from rhizotrons influenced by beech (B) and ash (A) after 475 days $(n=4)$. Means refer to densities in the middle part of the microcosms.

\begin{tabular}{|c|c|c|c|c|c|c|c|c|c|c|c|c|c|c|c|c|c|c|}
\hline \multirow[b]{4}{*}{ Soil taxa } & & & & & & & \multicolumn{6}{|c|}{ B- } & \multicolumn{6}{|c|}{$\mathrm{B}+$} \\
\hline & \multicolumn{2}{|c|}{ Beech } & \multicolumn{2}{|c|}{ Ash } & \multicolumn{2}{|c|}{ Beech $\times$ Ash } & \multicolumn{3}{|c|}{$\begin{array}{c}\text { A- } \\
\text { (Control) }\end{array}$} & \multicolumn{3}{|c|}{$\begin{array}{c}\text { A+ } \\
\text { (Ash) }\end{array}$} & \multicolumn{3}{|c|}{$\begin{array}{c}\text { A- } \\
\text { (Beech) }\end{array}$} & \multicolumn{3}{|c|}{$\begin{array}{c}\text { A+ } \\
\text { (Mixture) }\end{array}$} \\
\hline & $\boldsymbol{F}$ & $\boldsymbol{P}$ & $\boldsymbol{F}$ & $P$ & $\boldsymbol{F}$ & $\boldsymbol{P}$ & Mean & & SE & Mean & & SE & Mean & & SE & Mean & & SE \\
\hline & & & & & & & & & & & & & & & & & & \\
\hline Collembola $†$ & 0.20 & 0.6614 & 0.70 & 0.4177 & 0.20 & 0.6597 & 61.00 & \pm & 37.94 & 46.00 & \pm & 15.63 & 59.50 & \pm & 30.63 & 65.00 & \pm & 9.35 \\
\hline Sminthurida & 0.63 & 0.4418 & 2.70 & 0.1263 & 4.41 & 0.0576 & 54.00 & \pm & 13.36 & 14.50 & \pm & 5.48 & 12.25 & \pm & 6.84 & 21.50 & \pm & 12.07 \\
\hline Gamasida & 2.89 & 0.1148 & 0.00 & 0.9889 & 2.22 & 0.1624 & 167.25 & \pm & 116.19 & 21.50 & \pm & 4.03 & 49.50 & \pm & 17.29 & 43.50 & \pm & 9.46 \\
\hline Oribatida & 0.15 & 0.7023 & 0.28 & 0.6059 & 0.82 & 0.3820 & 10.50 & \pm & 2.50 & 8.50 & \pm & 2.99 & 9.75 & \pm & 2.29 & 10.25 & \pm & 0.48 \\
\hline Lumbricidae & 0.35 & 0.5626 & 2.40 & 0.1474 & 0.08 & 0.7884 & 2.00 & \pm & 0.91 & 2.50 & \pm & 1.19 & 0.50 & \pm & 0.29 & 1.00 & \pm & 0.41 \\
\hline
\end{tabular}




\title{
| Chapter 4
}

Global change belowground: impacts of elevated $\mathrm{CO}_{2}$, nitrogen, and summer drought on soil food webs and biodiversity

\author{
Nico Eisenhauer, Simone Cesarz, Robert Koller, \\ Kally Worm and Peter B. Reich
}

Published in Global Change Biology (2012) 18, $435-447$

doi: 10.1111/j.1365-2486.2011.02555.x

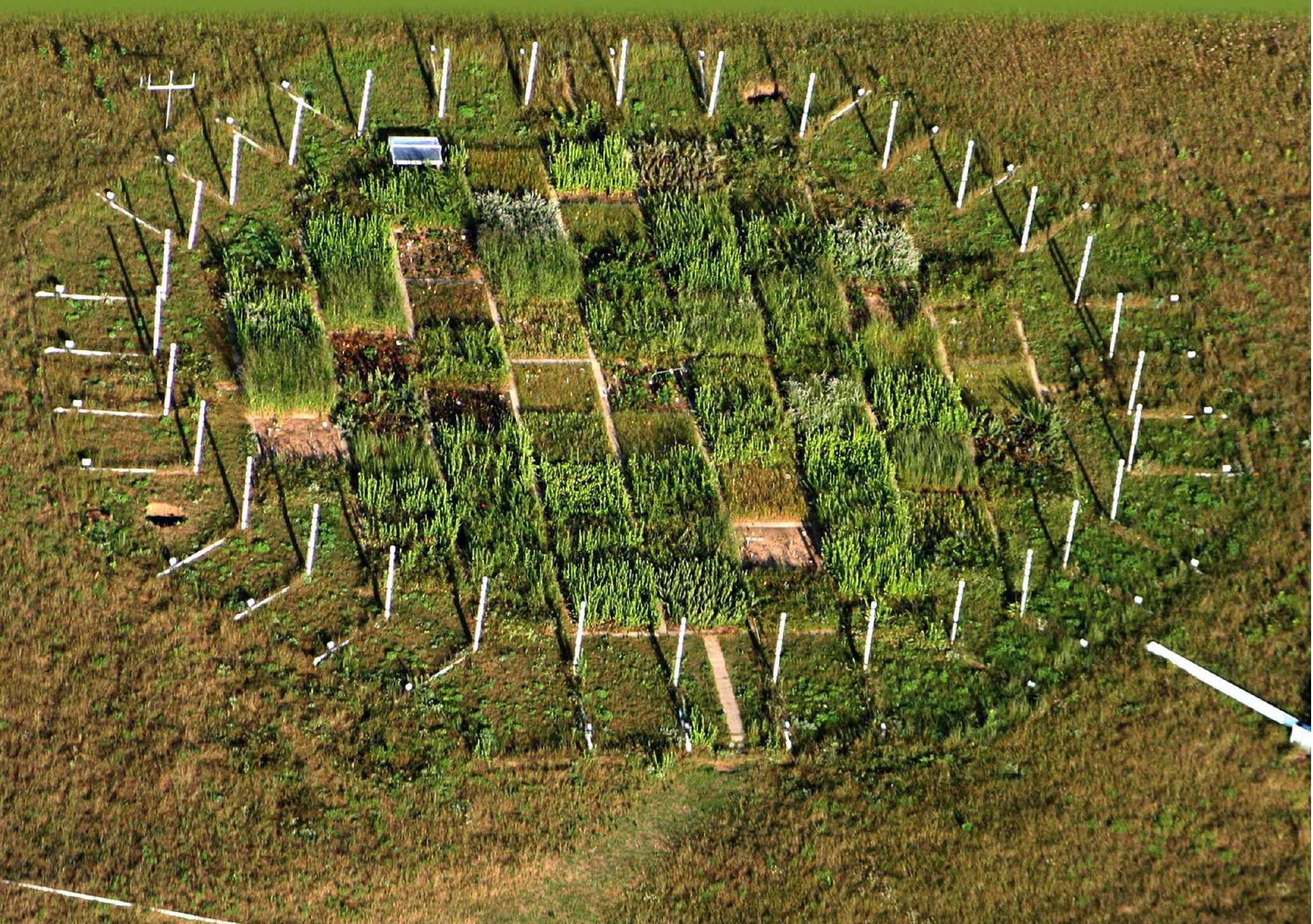




\section{Abstract}

The world's ecosystems are subjected to various anthropogenic global change agents, such as enrichment of atmospheric $\mathrm{CO}_{2}$ concentrations, nitrogen $(\mathrm{N})$ deposition and changes in precipitation regimes. Despite the increasing appreciation that the consequences of impending global change can be better understood if varying agents are studied in concert, there is a paucity of multi-factor long-term studies, particularly on belowground processes.

Here, we address this gap by examining the responses of soil food webs and biodiversity to enrichment of $\mathrm{CO}_{2}$, elevated $\mathrm{N}$ and summer drought in a long-term grassland study at Cedar Creek, Minnesota, USA (BioCON experiment). We use structural equation modeling (SEM), various abiotic and biotic explanatory variables, and data on soil microorganisms, protozoa, nematodes and soil microarthropods to identify the impacts of multiple global change effects on drivers below ground.

We found that long-term (13-year) changes in $\mathrm{CO}_{2}$ and $\mathrm{N}$ availability resulted in modest alterations of soil biotic food webs and biodiversi ty via several mechanisms, encompassing soil water availability, plant productivity and - m ost importantly - changes in rhizodeposition. Four years of manipulation of summer drought exerted surprisingly minor effects, only detrimentally affecting belowground herbivores, and ciliate protists at elevated N. Elevated $\mathrm{CO}_{2}$ increased microbial biomass and the density of ciliates, microarthropod detritivores and gamasid mites, most likely by fuelling soil food webs with labile C. Moreover, beneficial bottom-up effects of elevated $\mathrm{CO}_{2}$ compensated for detrimental elevated $\mathrm{N}$ effects on soil microarthropod taxa richness. By contrast, nematode taxa richness was lowest at elevated $\mathrm{CO}_{2}$ and elevated $\mathrm{N}$. Thus, enrichment of atmospheric $\mathrm{CO}_{2}$ concentrations and $\mathrm{N}$ deposition may result in taxonomically and functionally altere $d$, potentially simplified, soil communities. Detrimental effects of $\mathrm{N}$ deposition on soil biodive rsity underscore recent reports on plant community simplification. This is of particular con cern as soils house a considerable fraction of global biodiversity and ecosystem functions. 


\section{Introduction}

Humankind is changing the composition and functioni ng of ecosystems by causing alterations in global biochemistry and climate (Vitousek et al., 1997; Assessment Millenium Ecosystem (MA), 2005; IPCC, 2007). The burning of fossil fuel in the last two centuries led to a substantial increase of atmospheric $\mathrm{CO}_{2}$ concentrations with accelerating impacts on global climate and weather events (IPCC, 2007; Kerr, 2007). Concurrently, nitrogen (N) inputs increased dramatically due to fertilization and fossil fuel burning (Hungate et al., 1997; Vitousek et al., 1997). As a component of climate change the amount and timing of precipitation events are likely to change (Kerr, 2007), and globally the area affected by drought has increased since the 1970s (IPCC, 2007). All these global change agents are likely to have substantial effects on terrestrial ecosystems and knowledge on single-factor effects has proliferated considerably in the last two decades. However, given that multiple global change agents may interactively impact ecosystems (Sala, 2000; Reich, Knops, et al., 2001; Hungate et al., 2003; Dukes et al., 2005; IPCC, 2007), it remains largely unclear if results of previous single-factor experiments realistically model future responses (Luo et al., 2008; Butenschoen et al., 2011).

While aboveground responses to global change have elicited the most attention, belowground biota and functions are notoriously understudied (e.g., (West et al., 2006; Bardgett and Wardle, 2010). However, information on belowground responses to global changes is crucially important as soils house a considerable fraction of global biodiversity (Decaëns, 2010) and support a wide range of key ecosystem functions (Bardgett and Wardle, 2010). Moreover, a recent meta-analysis indicates that effects of $\mathrm{CO}_{2}$, warming and precipitation change with time, reinforcing the urgent need for long-term experiments (Blankinship et al., 2011).

Previous studies reported mainly positive effects of elevated $\mathrm{CO}_{2}$ on soil biota, although effects are assumed to attenuate with time (Blankinship et al., 2011). Several plant-mediated mechanisms underlying $\mathrm{CO}_{2}$ effects on soil biota are conceivable and not mutually exclusive. First, elevated $\mathrm{CO}_{2}$ often reduces plant stomatal conductance and thereby increases soil moisture content (Field et al., 1995), including in the BioCON experiment (Reich, 2009; Adair et al., 2011), which beneficially affects most soil biota (Coleman et al., 2004). Second, elevated $\mathrm{CO}_{2}$ may increase belowground translocation of assimila ted carbon by plants, which has been shown to increase microbial growth, biomass and change community composition (Blagodatskaya et al., 2010; Drigo et al., 2010). This effect can be due to elevated root biomass 
production and/or increased rhizodeposition (Jones et al., 2009). Third, increased shoot productivity at elevated $\mathrm{CO}_{2}$ (Reich, Knops, et al., 2001; Reich, Hobbie, et al., 2006b) may enhance the amount of aboveground litter input.

The above-mentioned mechanisms may also apply to effects of $\mathrm{N}$ addition and drought on plant productivity. While $\mathrm{N}$ addition has been shown to increase plant productivity in the BioCON experiment (Reich, Knops, et al., 2001; Reich, Hobbie, et al., 2006b), it may also decrease root exudation supporting lower microbial biomass (Dijkstra et al., 2005). Moreover, $\mathrm{N}$ fertilization was shown to decrease microbial enzyme activity (DeForest et al., 2004), though previous studies report inconsistent $\mathrm{N}$ addition effects on soil microorganisms (Niklaus and Körner, 1996; Zak et al., 2000; Dijkstra et al., 2005). Sjursen et al. (2005) reported enhanced microarthropod abundances in a subarctic ecosystem after application of NPK fertilizer, and assumed bottom-up effects of enhanced food availability.

Summer drought had mainly detrimental effects on soil enzyme activity and nematode density in the Old-Field Community, Climate and Atmosphere Manipulation Experiment in Oak Ridge, Tennesee, USA (Kardol et al. 2010). In contrast to effects of elevated $\mathrm{CO}_{2}$ and $\mathrm{N}$ addition, summer drought may also have direct effects on soil biota (Bardgett and Wardle, 2010) as most soil biota strongly depend on the availability of water (Coleman et al., 2004; Kardol et al., 2011). However, $\mathrm{CO}_{2}$, temperature and water availability interactively impacted soil ecosystem functioning in the study by Kardol et al. (2010). For instance, detrimental effects of temperature and drought on some nematode feeding groups materialized under ambient $\mathrm{CO}_{2}$ but not elevated $\mathrm{CO}_{2}$. Similarly, Chung et al. (2007) found that the com position and functioning of soil microbial communities depended on complex i nteractions between plant diversity, $\mathrm{CO}_{2}$ and $\mathrm{N}$ addition. Hoeksema et al. (2000) as well as Li et al. (2007) showed that nematode community structure and diversity significantly responded to interactions between $\mathrm{CO}_{2}$ and $\mathrm{N}$ under trembling aspen and wheat, respectively, pointing at a complex interplay between bottom-up and top-down forces in structuring soil b iotic communities. These studies present clear indications that the consequences of future global change agents may be better understood if studied in concert. Moreover, there is insufficient knowledge of the mechanisms of how different global change agents interactively impact soils (Bardgett and Wardle, 2010).

We address this critical gap by studying the most relevant soil food web components (microorganisms, protozoans, nematodes and microarthropods) in a well-established 
grassland global change experiment in Minnesota, USA (BioCON; Reich et al. 2001a), manipulating $\mathrm{CO}_{2}, \mathrm{~N}$ and summer drought in a complete factorial design. In order to gain a mechanistic understanding of how global change effects materialize below ground we use structural equation modeling (SEM).

According to the knowledge summarized above, we hypothesized that (1) elevated $\mathrm{CO}_{2}$ increases, (2) elevated $\mathrm{N}$ increases, and (3) summer drought decreases the abundance and diversity of soil biota. We expected beneficial impacts of $\mathrm{CO}_{2}$ and $\mathrm{N}$ to be due to increased resource availability for soil biota. In addition, elevated $\mathrm{CO}_{2}$ may positively affect soil biota by increasing soil water content. Moreover and most importantly, we hypothesized (4) the three global change agents to interactively affect soil biota. For instance, elevated $\mathrm{CO}_{2}$ may ameliorate drought effects, whereas elevated $\mathrm{N}$ may exacerbate drought effects by increasing plant productivity. By contrast, effects of elevated $\mathrm{CO}_{2}$ and $\mathrm{N}$ may be additive or greater-thanadditive as both factors and their interaction have been shown to increase plant productivity (Reich, Knops, et al., 2001; Reich, Hobbie, et al., 2006b). Moreover, N addition may decrease $\mathrm{N}$ constraints on soil microorganisms due to $\mathrm{CO}_{2}$ enrichment (Hu et al. 2001). Although many complex and plausible hypotheses about the interactions of these three factors could be stated, the complexity of the belowground network system is such that alternative hypotheses would often be equally plausible. This highlights the need for empirical examination of whether, and what kinds of interactions, occur in belowground communities.

\section{Materials and methods}

\section{The site and experimental design}

The present experiment was conducted in the framework of the BioCON experiment at the Cedar Creek LTER site in Minnesota (Reich et al. 2001a). The region has a continental climate with cold winters (mean January temperature $-11^{\circ} \mathrm{C}$ ) and warm summers (July temperature $22^{\circ} \mathrm{C}$ ) and mean annual precipitation of $660 \mathrm{~mm}$ (Reich et al. 2001a). The soils are sands (Typic Udipsamment, Nymore series) derived from sandy glacial outwash ( $94.4 \%$ sand, $2.5 \%$ clay). The BioCON experiment (including factorial combinations of $\mathrm{CO}_{2} \times \mathrm{N}$ treatments in plots varying in plant species composition and number) was established in 1997 on a level, secondary successional grassland after removing prior vegetation (Reich et al. 2001a). 
For the present experiment we used 48 of the original $2 \times 2 \mathrm{~m}$ BioCON plots, chosen randomly from the 64 plots that were initially planted with 9 species and now contain 6-7 species on average. We used a complete factorial design of 2 summer drought (precipitation) $\times 2 \mathrm{CO}_{2} \times$ $2 \mathrm{~N}$ treatments, each consisting of 6 unique replicates. Each plot was planted in 1997 with 9 species randomly selected from a pool of 16 herbaceous species representing 4 functional groups. The species pool was comprised of the $\mathrm{C}_{3}$ grasses Agropyron repens, Bromus inermis, Koeleria cristata, and Poa pratensis; the $\mathrm{C}_{4}$ grasses Andropogon gerardii, Bouteloua gracilis, Schizachyrium scoparium, and Sorghastrum nutans; the herbaceous forbs Achillea millefolium, Anemone cylindrica, Asclepias tuberosa, Solidago rigida; and the N-fixing legumes Amorpha canescens, Lespedeza capitata, Lupinus perennis, and Petalostemum villosum.

$\mathrm{CO}_{2}$ treatments consist of ambient and elevated $\mathrm{CO}_{2}$. Briefly, six circular areas ( $24 \mathrm{~m}$ diameter; Fig. 1) were randomly assigned, three each to ambient $\left(a \mathrm{CO}_{2}\right)$ and elevated $\mathrm{CO}_{2}\left(e \mathrm{CO}_{2} ;+180\right.$ ppm, $24 \mathrm{hrs} /$ day, early spring to late fall). The added $\mathrm{CO}_{2}$ is delivered using FACE technology (Hendrey et al. 1993). Within the central $20 \mathrm{~m}$ diameter zone in each ring, we established eight square plots for the present experiment. Plots are separated by a $20 \mathrm{~cm}$ walkway buffer and metal barriers $30 \mathrm{~cm}$ deep separate each plot. The elevated $\mathrm{CO}_{2}$ treatment has been ongoing since 1997.

Nitrogen was added to the surface of half the plots in each ring as $4 \mathrm{~g} \mathrm{~N} \mathrm{~m}^{-2} \mathrm{yr}^{-1}$ slow-release ammonium nitrate $\left(\mathrm{NH}_{4} \mathrm{NO}_{3}\right)$ in equal fractions in early May, June and July (a mbient $\mathrm{N}=a \mathrm{~N}$, elevated $\mathrm{N}=e \mathrm{~N}$ ). Annual net mineralization rates are roughly $3-4 \mathrm{~g} \mathrm{~N} \mathrm{~m}^{-2} \mathrm{y}^{-1}$ in grassland at Cedar Creek (Reich et al. 2001b). Thus, adding $4 \mathrm{~g} \mathrm{~N} \mathrm{~m}^{-2} \mathrm{y}^{-1}$ simulates doubling of available $\mathrm{N}$ in this system and serves to elucidate responses of ecosystems differing in soil $\mathrm{N}$ supply because of differences in fertility or N deposition. This treatment has been ongoing since 1997.

Half of the plots experienced summer drought via rain removal. For this treatment we used 24 portable $(2 \times 2 \mathrm{~m})$ rainout shelters (Fig. 1) to reduce both the number of precipitation events and total rainfall in the May-August period each year by approximately $-45 \%$. This represents current and a plausible but markedly different future climate scenario for Minnesota; the latter might occur as some predictions are for warmer and drier summers, with fewer rainfall events, that might collectively lead to an average of $10-40 \%$ lower soil moisture by 2100 (Wuebbles \& Hayhoe 2004; IPCC 2007). However, such predictions are 
highly uncertain and we view our treatment largely as a test of potential effects of diminished soil moisture rather than an assessment of effects of specific climate predictions.

Intercepted rain is channeled using gutters, and moved well outside each of the six approximately 60 -plot rings. Shelters were put into place during $45 \%$ of significant rainfall events between May and August (Table 1). Shelters were in place much less than $1 \%$ of the time (and nearly always under cloudy conditions and rarely during midday) and cumulatively reduce integrated photosynthetic photon flux density (May to August) by much less than $1 \%$ (approximately $0.05 \%$ ). The chambers cause minimal warming under conditions of use and given the limited time they are in place. This treatment has been ongoing since 2007 (details are given in Table 1).

\section{Samplings and measurements}

In August 2010, i.e., 13 years after establishment of the $\mathrm{CO}_{2}$ and $\mathrm{N}$ treatments, and after four years of summer drought treatment, we took soil samples to investigate treatment effects on soil biota. From each of the 48 plots we took three small soil samples (diameter $2 \mathrm{~cm}$, depth $6 \mathrm{~cm}$ ) and one larger soil sample (diameter $5 \mathrm{~cm}$, depth $6 \mathrm{~cm}$ ) using steel corers. The small soil samples were pooled in a plastic bag, carefully but thoroughly homogenized and stored at 4 ${ }^{\circ} \mathrm{C}$ until further processing. Large soil samples were kept intact and stored in plastic containers at $4{ }^{\circ} \mathrm{C}$ until further processing. Soil from the small samples was subdivided into three portions

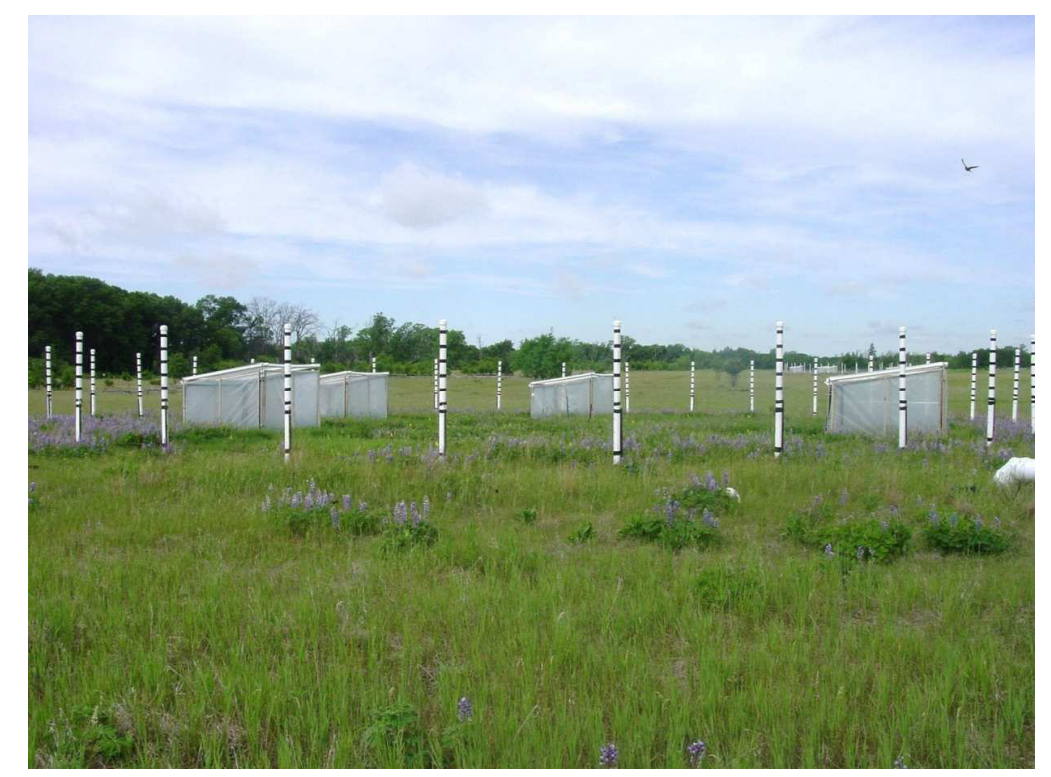

Figure 1 | Photograph showing one of the six FACE rings of the BioCON experiment in secondary successional grassland in Minnesota, USA (Reich et al., 2001a) with four of the 24 portable rain-out shelters temporarily in position above the plots of the precipitation. 
of approximately $15 \mathrm{~g}$ of soil (fresh weight) and used to measure soil microbial biomass, protozoans and nematodes, while large samples were extracted for soil microarthropods.

Before measurement of soil microbial biomass, soil sub-samples were sieved $(2 \mathrm{~mm})$ to remove larger roots, animals and stones (Anderson \& Domsch 1978). Microbial biomass C of approximately $5 \mathrm{~g}$ soil (fresh weight) was measured using an $\mathrm{O}_{2}$-microcompensation apparatus (Scheu 1992). Substrate induced respiration was calculated from the respiratory response to D-glucose for $10 \mathrm{~h}$ at $22{ }^{\circ} \mathrm{C}$ (Anderson \& Domsch 1978). Glucose was added according to preliminary studies to saturate the catabolic enzymes of microorganisms ( $4 \mathrm{mg}$ $\mathrm{g}^{-1}$ dry weight solved in $400 \mu$ l deionized water). The mean of the lowest three readings within the first $10 \mathrm{~h}$ was taken as maximum initial respiratory response (MIRR; $\mu \mathrm{O}_{2} \mathrm{~h}^{-1} \mathrm{~g}^{-1}$ soil dry weight) and microbial biomass ( $\mu \mathrm{g} \mathrm{C}^{-1}$ soil dry weight) was calculated as $38 \times \mathrm{MIRR}$ (Beck et al. 1997).

Total numbers of protozoa (i.e., active and encysted forms of amoebae, ciliates and flagellates) were enumerated by a modified most probable number method (Darbyshire et al. 1974). Briefly, $5 \mathrm{~g}$ fresh weight of soil was suspended in $20 \mathrm{ml}$ sterile Neff's modified amoebae saline (NMAS; Page 1976) and gently shaken (70 rpm) for $20 \mathrm{~min}$ on a vertical shaker. Threefold dilution series with nutrient broth (Merck, Darmstadt, Germany) and NMAS at 1:9 v/v were prepared in 96-well microtiter plates (VWR, Darmstadt, Germany) with four replicates each.

Table 1 | Details of the precipitation experiment. Given are the period of precipitation manipulation in each year, the amount of precipitation accumulated within the respective periods, and the percentage of precipitation removed using rain-out shelters (Fig. 1).

\begin{tabular}{ccccc}
\hline Year & Start & End & Precipitation [mm] & Percent removed \\
\hline 2007 & 30-May-2007 & 5-Aug-07 & 144 & 37 \\
2008 & 13-May-2008 & 6-Aug-08 & 235 & 47 \\
2009 & 9-May-2009 & 8-Aug-09 & 185 & 46 \\
2010 & 7-May-2010 & 10-Aug-10 & 445 & 49 \\
\hline
\end{tabular}


The microtiter plates were incubated at $15^{\circ} \mathrm{C}$ in darkness and the wells were inspected for presence of protozoa using an inverted microscope at 100x and 200x magnification (Nikon, Eclipse TE 2000-E, Tokyo, Japan) after 3, 6, 11, 19 and 26 days. Densities of protozoa were calculated according to Hurley \& Roscoe (1983) and related to g soil dry weight.

Nematodes were extracted from $10 \mathrm{~g}$ soil (fresh weight) using a modified Baermann method (Ruess 1995). After an extraction time of $30 \mathrm{~h}$ nematodes were preserved in $4 \%$ formaldehyde, counted and related to $g$ soil dry weight. Subsequently, $10 \%$ of the individuals (but not less than 100 individuals, if possible) were additionall y identified to family level or if necessary to genus level and assigned to the trophic groups bacterial feeders, fungal feeders, omnivores, plant feeders and predators according to Yeates et al. (1993).

Soil microarthropods were extracted by heat (Kempson et al. 1963), collected in diluted glycerol, and transferred into ethanol (70\%) for storage. Soil animals were determined following Gisin (1960), Fjellberg (1980), Schaefer (2000), Hopkin (2007) and Krantz \& Walter (2009) and counted (abundance $\mathrm{m}^{-2}$ ). Astigmatic and prostigmatic were pooled without further determination; however, for the calculation of microarthropod taxa richness morphospecies were distinguished.

\section{Explanatory variables}

In order to investigate how different global change agents influence soil biota, we measured several explanatory variables contemporary to the samplings explained above. In August 2010, we determined plant community shoot, root biomass and surface litter biomass ( $\mathrm{g} \mathrm{m}^{-2}$; for detailed methods see e.g., Reich et al. 2006), and realized plant species richness. Briefly, aboveground biomass was harvested by clipping a $10 \mathrm{~cm} \times 100 \mathrm{~cm}$ strip just above the soil surface. Total root biomass was sampled at 0-20 cm depth by taking three soil cores of $5 \mathrm{~cm}$ in diameter. Further, $\mathrm{C}$ and $\mathrm{N}$ concentrations in plant shoot and root tissue were determined (Reich et al. 2001a). Gravimetric soil water content [\%] was measured from soil samples for microbial analyses. Moreover, $\mathrm{N}$ mineralization $\left[\mathrm{mg} \mathrm{kg}^{-1} \mathrm{~d}^{-1}\right.$ ] was determined according to West et al. (2006). These variables were determined to test the hypotheses that effects of global change agents on soil biota are due to chang es (i) in soil water content, (ii) aboveground productivity (shoot and litter biomass), (iii) belo wground productivity (root biomass), (iv) quality of plant inputs ( $\mathrm{N}$ concentration of plant $\mathrm{t}$ issue, $\mathrm{N}$ mineralization), or indirectly $(\mathrm{v}$ ) in 
root rhizodeposits. Since we did not measure root rhizodeposits directly, we followed the approach by Lamb et al. (2011) using structural equation modeling (see below for more details). Treatment effects on explanatory variables have been or will be published elsewhere (e.g., Reich et al. 2001a, 2006; West et al. 2006; Adair et al. 2009; Reich 2009; P. B. Reich, unpublished data) and thus are not given in the pre sent paper; however, they were included in the SEM (see below).

\section{Statistical analyses}

Data on soil water content, soil microbial biomass, soil animal densities and taxa richness were log-transformed to meet the assumptions of parametric statistical tests (normality and homoscedasticity of errors). Means ( \pm standard error) presented in text, Fig. 2 and Table 2 were calculated using non-transformed data. Analysis of variance (ANOVA) was performed to test the effects of $\mathrm{CO}_{2}$ (ambient and elevated), $\mathrm{N}$ (ambient and elevated) and summer drought (D; ambient and precipitation reduction) and interactions on soil microbial biomass, abundance of amoebae, ciliates, flagellates, total nematodes, plant feeding nematodes, bacterial feeding nematodes, fungal feeding nematodes, omnivorous nematodes, carnivorous nematodes, collembolans, oribatid mites, gamasid mites, astigmatic and prostigmatic mites, microarthropod herbivores (mainly Thysanoptera), and on the taxa richness of nematodes and microarthropods. The effect of $\mathrm{CO}_{2}$ was tested against the random effect of ring nested within $\mathrm{CO}_{2}$ (Reich et al. 2001a). $\mathrm{CO}_{2}, \mathrm{~N}$ and $\mathrm{D}$ treatments were independent from each other. We did not correct for multiple statistical tests considering the mathematical and logical argumentation by Moran (2003). Some models were improved based on AIC by removing nonsignificant independent variables or interactions (Table 3).

In addition to ANOVA, we used SEM to investigate how global change agents affect soil food webs and biodiversity. The results of the ANOVA approach served as the hypothetical base for the initial SEM model. In the initial model we only used exogenous and endogenous variables which had significant effects in the ANOVA approach and which were significantly affected or showed distinct tendencies due to the low number of replicates ( $n=48$; Grace 2006). Moreover, predicted causal relationships between variables were based on prior knowledge on effects of global change agents on the performance of plants and soil biota. The adequacy of the model was determined via $\chi^{2}$ tests, AIC and RMSEA. Adequate model fits are indicated by non-significant $\chi^{2}$ tests $(P>0.05)$, low AIC and low RMSEA $(<0.05)$ (Grace 2006; Arbuckle 
2010). Two separate models were determined for soil food web structure (based on abundance and biomass data) and soil biodiversity (i.e., nematode taxa richness and microarthropod taxa richness). Due to the complete factorial design global change agents were uncorrelated to each other. Both bottom-up and top-down relationships between soil food web components were tested (not shown). Model modification indices were used to improve the models; however, only scientifically sound relationships were considered (Grace 2006).

As stated above, we tested five hypothetical pathways regarding how global change agents affect soil biota; four of them were measured directly (aboveground productivity, belowground productivity, input quality and soil wa ter content). Since effects through altered rhizodeposits are also likely, we followed the approach by Lamb et al. (2011) to assume that any effects of that type would be either covered by direct paths from global change agents to endogenous variables or should have been captured by the model modification indices. SEM was performed using Amos 5 (Amos Development Corporation, Crawfordville, FL, USA).

\section{Results}

\section{ANOVA results}

Overall, manipulation of $\mathrm{CO}_{2}, \mathrm{~N}$ and summer drought exerted modest effects on soil biota, significantly affecting the abundance/biomass of 7, 5 and 2 out of 14 groups of soil biota, respectively (Table 3). The interaction between $\mathrm{CO}_{2}$ and $\mathrm{N}$ accounted for 5 out of 6 significant $(P<0.1)$ interactions between the investigated global change agents. Microbial biomass (mean $\pm \mathrm{SE}: 478.2 \pm 20.5 \mu \mathrm{g} \mathrm{C}_{\text {mic }} \mathrm{g}^{-1}$ soil dry weight) was significantly higher at $e \mathrm{CO}_{2}$ than at $a \mathrm{CO}_{2}$ (+28\%; Table 3), which was mainly due to low microbial biomass at $a \mathrm{CO}_{2}$ and $e \mathrm{~N}$ (Fig. 2a). While flagellates $\left(16,309 \pm 2,542\right.$ ind. $\mathrm{g}^{-1}$ soil $\left.\mathrm{dw}\right)$ and amoebae $\left(8,962 \pm 1,017\right.$ ind. $\mathrm{g}^{-1}$ soil $d w$ ) were not significantly affected by our treatments, ciliate abundance ( $27 \pm 4$ ind. $g^{-1}$ soil $\mathrm{dw}$ ) was considerably higher at $e \mathrm{CO}_{2}$ than at $a \mathrm{CO}_{2}(+83 \%)$. Moreover, ciliate abundance did not differ between $a \mathrm{~N}$ and $e \mathrm{~N}$ at ambient precipitation, but was significantly lower at $e \mathrm{~N}$ in comparison to $a \mathrm{~N}$ in treatments with reduced precipitation (-39\%). Generally, total nematode abundance ( $20 \pm 2$ ind. $\mathrm{g}^{-1}$ soil $\mathrm{dw}$ ) was little affected by our treatments (Table 3 ). However, this masks different responses of contrasting nematode functional groups. The abundance of 

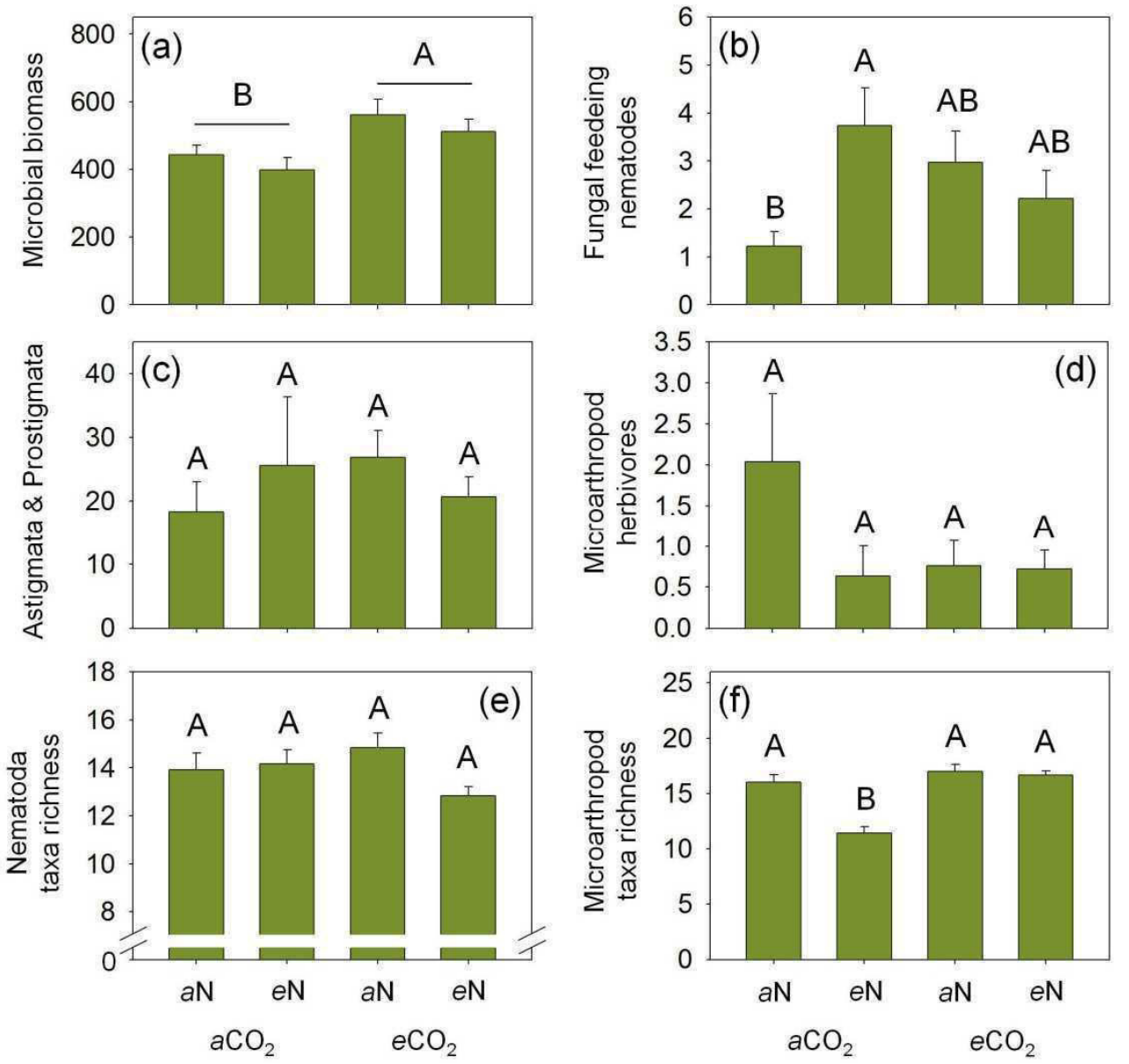

Figure 2 | Microbial biomass [ $\mu \mathrm{g} \mathrm{Cmic}^{-1}$ soil dw] (a), abundance of fungal feeding nematodes [ind. $\mathrm{g}^{-1}$ soil $\mathrm{dw}$ ] (b), abundance of Astigmata and Prostigmata [ind. $\mathrm{m}^{-2} \times 1000$ ] (c), microarthropod herbivores [ind. $\mathrm{m}^{-2} \times 1000$ ] (d), Nematoda taxa richness (e), and soil microarthropod taxa richness ( $\mathrm{f}$ ) as affected by nitrogen addition ( $a \mathrm{~N}=$ ambient $\mathrm{N}, e \mathrm{~N}=$ elevated $\mathrm{N})$ and $\mathrm{CO}_{2}\left(a \mathrm{CO}_{2}=\right.$ ambient $\mathrm{CO}_{2}, e \mathrm{CO}_{2}=$ elevated $\left.\mathrm{CO}_{2}\right)$ in secondary successional grassland in Minnesota, USA. Means \pm SE. Bars with varying letters differ significantly (Tukey's HSD test, $P<$ 0.05).

predator nematodes $\left(0.58 \pm 0.10\right.$ ind. $\mathrm{g}^{-1}$ soil $\left.\mathrm{dw}\right)$ decreased significantly at $e \mathrm{~N}$ in comparison to $a \mathrm{~N}(-62 \%)$. By contrast, the abundance of nematode fungal feeders $\left(2.53 \pm 0.33\right.$ ind. $\mathrm{g}^{-1}$ soil dw) was significantly higher at $e \mathrm{~N}$ in comparison to $a \mathrm{~N} \mathrm{(+206 \% )} \mathrm{at} a \mathrm{CO}_{2}$, whereas the $\mathrm{N}$ treatment had no significant effect at $e \mathrm{CO}_{2}$ (Fig. 2b). Abundance of collembolans $(3,523 \pm 526$ ind. $\left.\mathrm{m}^{-2}\right)$ and gamasid mites $\left(840 \pm 143\right.$ ind $\left.\mathrm{m}^{-2}\right)$ increased significantly at $e \mathrm{CO}_{2}(+64 \%$ and $+143 \%$, respectively; Table 3 ). There was also a strong but statistically marginal increase in oribatid mite abundance $\left(9,809 \pm 1,273\right.$ ind. $\left.\mathrm{m}^{-2}\right)$ at elevated $\mathrm{CO}_{2}(+87 \%)$, resulting in an overall tendency of increased microarthropod detritivore abundance $(+81 \%$; collembolans and oribatid mites). While the abundance of astigmatic and prostigmatic mites $(22,854 \pm 3,156$ ind. $\mathrm{m}^{-2}$ ) was little affected by $\mathrm{CO}_{2}$ at $e \mathrm{~N}$, it increased at $e \mathrm{CO}_{2}$ in comparison to $a \mathrm{CO}_{2}$ 
Table 2 Mean (SE in brackets) biomass (microorganisms), abundance (soil animals), and taxa richness of soil bi ota in the varying $\mathrm{CO}_{2}$ (ambient [aCO$\left.{ }_{2}\right]$ and elevated $\left.\left[e \mathrm{CO}_{2}\right]\right), \mathrm{N}$ (ambient $[\mathrm{aN}]$ and elevated $[a \mathrm{~N}]$ ), and summer drought (ambient $\left[a \mathrm{H}_{2} \mathrm{O}\right]$ and reduced precipitation $\left[r \mathrm{H}_{2} \mathrm{O}\right]$ ) treatments

\begin{tabular}{|c|c|c|c|c|c|c|c|c|}
\hline & \multicolumn{4}{|c|}{$e \mathrm{CO}_{2}$} & \multicolumn{4}{|c|}{$\mathrm{aCO}_{2}$} \\
\hline & \multicolumn{2}{|c|}{$a \mathbf{N}$} & \multicolumn{2}{|c|}{$e N$} & \multicolumn{2}{|c|}{$a \mathrm{~N}$} & \multicolumn{2}{|c|}{$e N$} \\
\hline & $r \mathrm{H}_{2} \mathrm{O}$ & $a \mathrm{H}_{2} \mathrm{O}$ & $r \mathrm{H}_{2} \mathrm{O}$ & $a \mathrm{H}_{2} \mathrm{O}$ & $r \mathrm{H}_{2} \mathrm{O}$ & $a \mathrm{H}_{2} \mathrm{O}$ & $r \mathrm{H}_{2} \mathrm{O}$ & $a \mathrm{H}_{2} \mathrm{O}$ \\
\hline \multicolumn{9}{|l|}{ Microorganisms } \\
\hline Microbial biomass & $541(73)$ & $580(66)$ & $520(56)$ & $503(50)$ & 428 (49) & $458(32)$ & $340(50)$ & $455(50)$ \\
\hline \multicolumn{9}{|l|}{ Protozoa } \\
\hline Flagellata & 11619 (2293) & 13025 (3587) & $22629(15196)$ & 20256 (6593) & 13801 (5504) & $22983(10158)$ & 11636 (4057) & $14518(3214)$ \\
\hline Amoebae & 8211 (3995) & 8197 (2369) & $8533(2543)$ & $10316(2102)$ & 7850 (3537) & $8740(2683)$ & 9864 (4455) & $9983(2108)$ \\
\hline Ciliates & $42(16)$ & $36(7)$ & $28(11)$ & $31(10)$ & $22(7)$ & $17(8)$ & $12(5)$ & $24(5)$ \\
\hline \multicolumn{9}{|l|}{ Microfauna } \\
\hline Nematoda (total) & $21(4)$ & $19(2)$ & $19(4)$ & $20(6)$ & $15(3)$ & $20(3)$ & $24(3)$ & $21(3)$ \\
\hline Plant feeders & $2(1)$ & $3(1)$ & $2(1)$ & $3(2)$ & $2(1)$ & $4(2)$ & $2(1)$ & $3(2)$ \\
\hline Bacterial feeders & $13(3)$ & $10(2)$ & $12(3)$ & $13(4)$ & $7(2)$ & $11(2)$ & $12(3)$ & $10(1)$ \\
\hline Fungal feeders & $4(2)$ & $3(1)$ & $2(1)$ & $3(1)$ & $1(1)$ & $2(1)$ & $4(2)$ & $4(1)$ \\
\hline Omnivores & $3(1)$ & $3(1)$ & $3(1)$ & $2(1)$ & $3(1)$ & $4(1)$ & $4(1)$ & $3(1)$ \\
\hline Predators & $1(1)$ & $1(1)$ & $1(1)$ & $1(1)$ & $1(1)$ & $2(1)$ & $1(1)$ & $1(1)$ \\
\hline \multicolumn{9}{|l|}{ Microarthopods } \\
\hline Collembola & $4072(862)$ & 3988 (987) & $6617(2661)$ & $2800(366)$ & $934(306)$ & $4836(1692)$ & $2800(1912)$ & 2121 (970) \\
\hline Oribatida & $10096(2101)$ & $13743(2810)$ & 16204 (5959) & $11114(3466)$ & $8060(2652)$ & $8144(3152)$ & 6787 (4388) & $4326(2321)$ \\
\hline Microarthropod detritivores & $14168(2627)$ & $17731(3436)$ & $22821(6956)$ & 13914 (3589) & $8994(2762)$ & $12980(4155)$ & 9587 (4616) & 6447 (2929) \\
\hline Gamasida & $679(252)$ & $1612(594)$ & 1273 (599) & $1188(503)$ & $764(218)$ & $679(252)$ & $85(85)$ & $425(243)$ \\
\hline Astigmata \& Prostigmata & $26129(7857)$ & $27571(4348)$ & $25196(5768)$ & 16204 (1448) & 18664 (7394) & 17985 (6535) & $25790(15166)$ & $25366(16716)$ \\
\hline Herbivores & $594(333)$ & $933(533)$ & $509(263)$ & $933(382)$ & 1103 (499) & 2970 (1569) & $255(174)$ & $1018(720)$ \\
\hline \multicolumn{9}{|l|}{ Soil animal biodiversity } \\
\hline Nematoda taxa richness & $15(1)$ & $15(1)$ & $13(1)$ & $13(1)$ & $14(1)$ & $14(1)$ & $14(1)$ & $15(1)$ \\
\hline Microarthropod taxa richness & $17(2)$ & $18(2)$ & $17(2)$ & $17(2)$ & $15(2)$ & $17(3)$ & $12(3)$ & $11(2)$ \\
\hline
\end{tabular}


Table 3 ANOVA table of $F$ and $P$ values on the effects of $\mathrm{CO}_{2}$ (ambient and elevated), $\mathrm{N}$ (ambient and elevated), summer drought (ambient and reduced precipitation), and all possible interactions on the biomass (micro organisms), abundance (soil animals), and taxa rich ness of soil biota.

\begin{tabular}{|c|c|c|c|c|c|c|c|c|c|c|c|c|c|c|c|c|c|c|c|}
\hline & \multicolumn{2}{|c|}{$\mathrm{CO}_{2}$} & & \multicolumn{2}{|c|}{$\operatorname{Ring}\left(\mathrm{CO}_{2}\right)$} & \multicolumn{2}{|r|}{$\mathrm{N}$} & \multicolumn{3}{|c|}{ Drought (D) } & & \multicolumn{2}{|c|}{$\mathrm{CO}_{2} \times \mathrm{N}$} & \multicolumn{2}{|c|}{$\mathrm{CO}_{2} \times \mathrm{D}$} & \multicolumn{2}{|c|}{$\mathrm{N} \times \mathrm{D}$} & \multicolumn{2}{|c|}{$\mathrm{CO}_{2} \times \mathrm{N} \times \mathrm{D}$} \\
\hline & $F$ & $P$ & & $F$ & $P$ & $F$ & $P$ & & $F$ & $P$ & & $F$ & $P$ & $F$ & $P$ & $F$ & $P$ & $F$ & $P$ \\
\hline \multicolumn{20}{|l|}{ Microorganisms } \\
\hline Microbial biomass & 13.23 & 0.0226 & $\uparrow$ & 0.70 & 0.5972 & 1.89 & 0.1772 & \multicolumn{4}{|c|}{ excluded } & \multicolumn{2}{|c|}{ excluded } & exc & uded & \multicolumn{2}{|c|}{ excluded } & \multicolumn{2}{|c|}{ excluded } \\
\hline \multicolumn{20}{|l|}{ Protozoa } \\
\hline Flagellata & \multicolumn{2}{|c|}{ excluded } & & 0.90 & 0.4907 & \multicolumn{2}{|c|}{ excluded } & & 1.85 & 0.1812 & & \multicolumn{2}{|c|}{ excluded } & exc & uded & \multicolumn{2}{|c|}{ excluded } & \multicolumn{2}{|c|}{ excluded } \\
\hline Amoebae & \multicolumn{2}{|c|}{ excluded } & & 0.51 & 0.7643 & 1.97 & 0.1684 & & 2.00 & 0.1653 & & \multicolumn{2}{|c|}{ excluded } & exc & uded & \multicolumn{2}{|c|}{ excluded } & \multirow{2}{*}{\multicolumn{2}{|c|}{$\begin{array}{l}\text { excluded } \\
\text { excluded }\end{array}$}} \\
\hline Ciliates & 7.65 & 0.0528 & $\uparrow$ & 0.79 & 0.5393 & 0.01 & 0.9163 & & 0.45 & 0.5079 & & 1.49 & 0.2296 & exc & uded & 4.23 & 0.0466 & & \\
\hline \multicolumn{20}{|l|}{ Microfauna } \\
\hline Nematoda (total) & 0.04 & 0.8454 & & 5.01 & 0.0025 & 0.34 & 0.5649 & & 0.01 & 0.9420 & & 2.17 & 0.1495 & 0.32 & 0.5747 & 1.52 & 0.2247 & exc & uded \\
\hline Plant feeders & 2.41 & 0.1959 & & 2.13 & 0.0944 & \multicolumn{2}{|c|}{ excluded } & & \multicolumn{2}{|c|}{ excluded } & \multicolumn{3}{|c|}{ excluded } & exc & uded & exc & uded & exc & uded \\
\hline Bacterial feeders & 0.23 & 0.6573 & & 3.95 & 0.0092 & 0.73 & 0.3986 & & 0.00 & 0.9552 & & 0.63 & 0.4340 & 0.40 & 0.5288 & 0.42 & 0.5204 & 3.14 & 0.0850 \\
\hline Fungal feeders & 0.06 & 0.8188 & & 6.01 & 0.0006 & 1.94 & 0.1717 & & exc & luded & & 8.99 & 0.0047 & exc & uded & exc & uded & exc & uded \\
\hline Omnivores & excl & uded & & 2.99 & 0.0298 & 0.73 & 0.3979 & & 1.90 & 0.1754 & & exc & luded & exc & uded & 2.65 & 0.1116 & exc & uded \\
\hline Predators & 2.49 & 0.1910 & & 1.21 & 0.3218 & 9.08 & 0.0045 & $\downarrow$ & 0.36 & 0.5385 & & exc & luded & exc & uded & 2.57 & 0.1172 & exc & uded \\
\hline Microarthropods & & & & & & & & & & & & & & & & & & & \\
\hline Collembola & 7.85 & 0.0487 & $\uparrow$ & 0.75 & 0.5663 & exc & luded & & exc & luded & & exc & luded & exc & uded & exc & uded & exc & uded \\
\hline Oribatida & 2.29 & 0.2044 & & 10.26 & $<.0001$ & exc & luded & & exc & luded & & exc & luded & exc & uded & exc & uded & exc & uded \\
\hline Microarthropod detrit. & 2.81 & 0.1688 & & 5.84 & 0.0008 & 0.80 & 0.3754 & & exc & luded & & exc & luded & exc & uded & exc & uded & exc & uded \\
\hline Gamasida & 11.14 & 0.0324 & $\uparrow$ & 0.57 & 0.6875 & 0.37 & 0.5446 & & 0.97 & 0.3323 & & 0.83 & 0.3685 & 0.28 & 0.5999 & 0.21 & 0.6468 & 1.77 & 0.1912 \\
\hline Astig. \& Prostig. & 0.01 & 0.9129 & & 10.23 & $<.0001$ & 0.78 & 0.3843 & & 0.20 & 0.6581 & & 4.56 & 0.0396 & 0.11 & 0.7414 & 0.06 & 0.8048 & 1.72 & 0.1974 \\
\hline Herbivores & 0.31 & 0.6076 & & 6.50 & 0.0004 & 3.01 & 0.0905 & $\downarrow$ & 5.48 & 0.0244 & $\downarrow$ & 4.58 & 0.0387 & exc & uded & exc & uded & exc & uded \\
\hline Soil animal biodiversity & & & & & & & & & & & & & & & & & & & \\
\hline Nematoda richness & 0.03 & 0.8703 & & 6.10 & 0.0006 & 6.01 & 0.0187 & $\downarrow$ & exc & luded & & 2.72 & 0.1069 & exc & uded & exc & uded & exc & uded \\
\hline Microarthropod richness & 1.75 & 0.2533 & & 8.01 & $<.0001$ & 4.93 & 0.0323 & $\downarrow$ & exc & luded & & 4.17 & 0.0479 & exc & uded & exc & uded & exc & uded \\
\hline
\end{tabular}


at $a \mathrm{~N}(+46 \%$; Fig. $2 \mathrm{c})$. The abundance of herbivores $\left(1,049 \pm 255\right.$ ind. $\left.\mathrm{m}^{-2}\right)$ was decreased significantly in the reduced precipitation treatment (-58\%). Moreover, microarthropod herbivore abundance was higher, though not signific antly, at $a \mathrm{CO}_{2}$ and $a \mathrm{~N}$ in comparison to all other treatments (Fig. 2d).

Nematode taxa richness (14 \pm 1 taxa) and microarthropod taxa richness (16 \pm 1 taxa) decreased significantly at elevated N (-7\%; and $-15 \%$, respectively; Table 3, Fig. 2e,f). Microarthropod taxa richness decreased significantly at $\mathrm{eN}$ when $\mathrm{CO}_{2}$ was at ambient levels ($28 \%$ ), but it was virtually unaffected by $e \mathrm{~N}$ at $e \mathrm{CO}_{2}$ (Fig. 2f). By contrast, the detrimental effect of $\mathrm{N}$ addition on nematode taxa richness was most pronounced at e $\mathrm{CO}_{2}$ (-13\%; Fig. 2e).

\section{SEM results}

The final models adequately fit the data on soil fo od web $\left(\chi^{2}{ }_{13}=12.68, P=0.47 ; \mathrm{AIC}=58.68\right.$; RMSEA $=<.001)$ and soil biodiversity $\left(\chi^{2} 15=6.93, P=0.96 ;\right.$ AIC $\left.=48.93 ; \operatorname{RMSEA}<.001\right)$ (standardized path coefficients are given in Fig. 3, unstandardized path coefficients are given in Fig. S1). $\mathrm{CO}_{2}$ was the only exogenous variable remaining in the final soil food web model (Table S1; Fig. 3a) supporting the ANOVA results. Elevated $\mathrm{CO}_{2}$ increased gravimetric soil water content, soil microbial biomass, and the abundances of soil microarthropod detritivores and ciliates (the latter marginally significantly). Increased microbial biomass and ciliate abundance increased the abundance of amoebae, although these relationships were marginally significant. Increasing abundance of microarthropod detritivores increased the abundance of gamasid mites. The remaining relationships between exo- and endogenous variables were not significant but improved the fit of the model (Table S1; Fig. 3a). In the final soil biodiversity model, $e \mathrm{CO}_{2}$ had direct positive effects on soil water content, shoot biomass, microbial biomass and soil microarthropod taxa richness (Table S1; Fig. 3b). By contrast, eN negatively influenced soil microarthropod taxa richness. Incre ased shoot biomass led to increased litter biomass on the soil surface. Moreover, increased litter biomass positively influenced soil microarthropod taxa richness, whereas nematode taxa richness decreased. Microarthropod taxa richness was negatively related to soil water content, although this relationship was only marginally significant. The remaining relationships between exo- and endogenous variables were not significant but improved the fit of the model (Table S1; Fig. 3b). The final model 

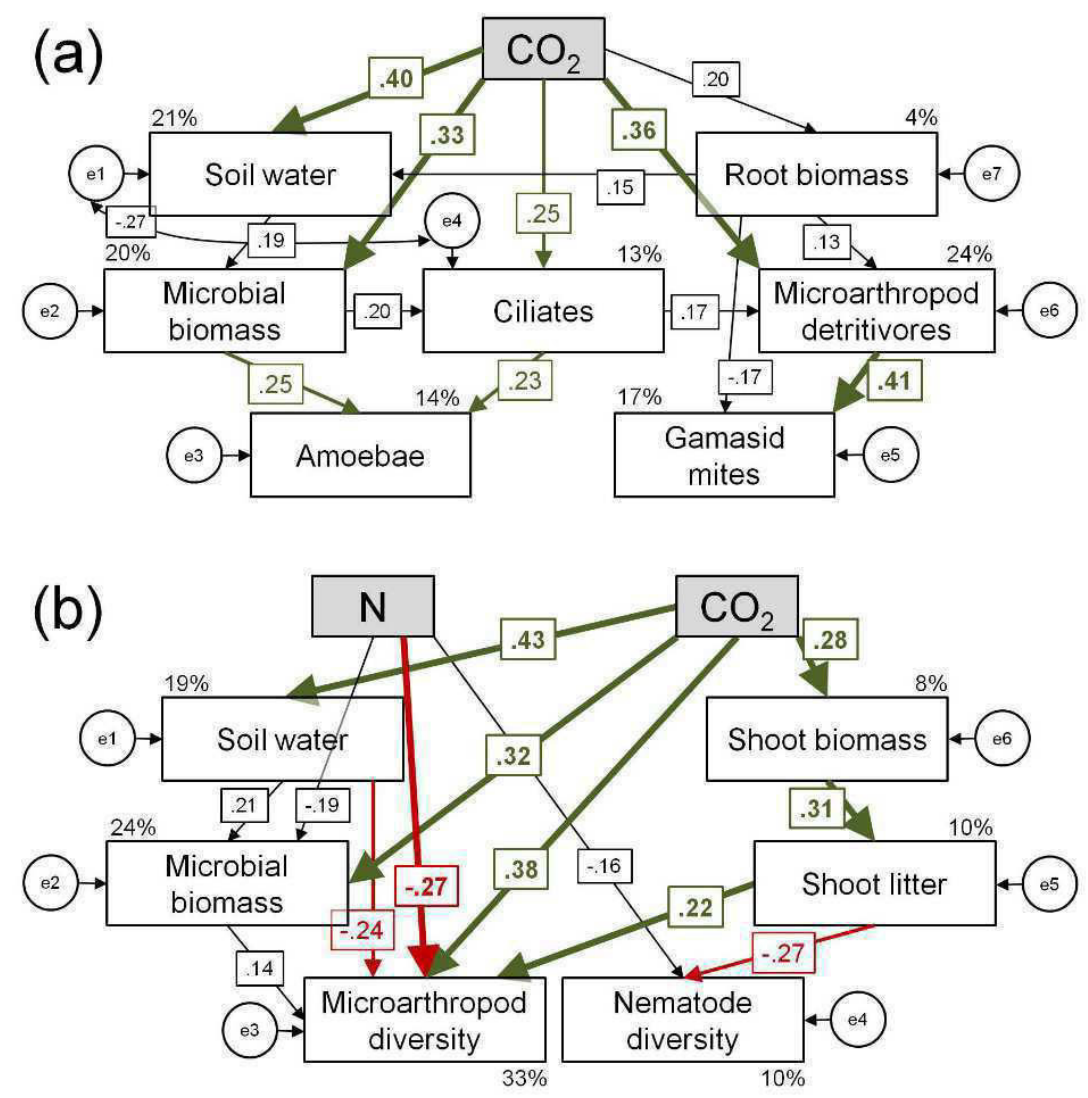

Figure 3 | Structural equation models of global change effects on soil biota in secondary successional grassland in Minnesota, USA. (a) Causal influences of elevated $\mathrm{CO}_{2}$ (exogenous variable; grey rectangle) on soil water content, root biomass productivity, microbial biomass and abundance of soil animals (endogenous variab les; white rectangle). The model fit the data well: $\chi^{2}{ }_{13}=12.68, P=0.47$. Numbers on arrows are standardized path coefficients (equivalent to correlation coefficients). Width of the arrows indicated the strength of the causal influence: green or red, bold arrows indicate significant standardized path coefficients $(P<0.05)$, green or red arrows indicate marginally significant path coeffic ients $(0.05<P<0.1)$, black, single-headed arrows indicate non-significant path coefficients $(P>0.1)$. Green arrows indicate significant and marginally significant positive relationships and red arrows significant and margin ally significant negative relationships. Circles in dicate error terms (e1-e7); double-headed errors indicate correlations between error terms. Percentages close to endogenous variables indicate the variance explained by the model $\left(R^{2}\right)$. (b) Causal influences of elevated $\mathrm{N}$ and $\mathrm{CO}_{2}$ (exogenous variables) on soil water content, shoot biomass productivity, shoot litter, microbial biomass and taxa richness of soil microarthropods and nematodes (endogenous variables; white rectangle). The model fit the data well: $\chi^{2} 15=6.93, P=0.96$.

explained a large proportion of the variance in soil microarthropod taxa richness (33\%), whereas it explained only $10 \%$ of nematode taxa richness. Both final SEM models indicate that direct pathways between global change agents and soil food web components and biodiversity, which are assumed to represent changes in rhizodeposition, were more important than indirect connections via changes in soil water content, or shoot and root productivity. 

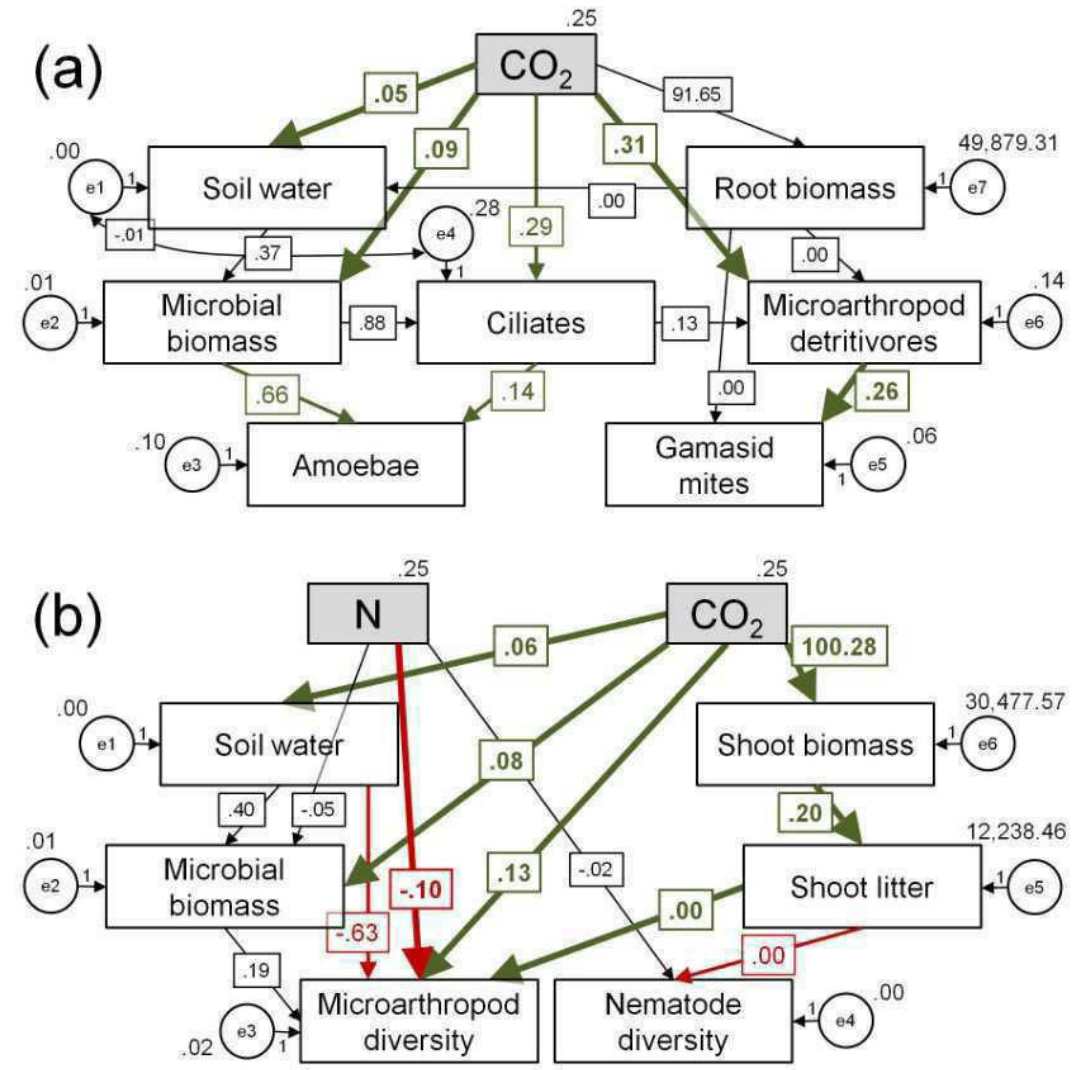

Figure S1 | Structural equation models of global change effects on soil biota. (a) Causal influences of elevated $\mathrm{CO}_{2}$ (exogenous variable; grey rectangle) on soil water content, root biomass productivity, microbial biom ass and abundance of soil animals (endogenous variables; white rectangle). The model fit the data well: $\chi^{2} 13=$ $12.68, P=0.47$. (b) Causal influences of elevated $\mathrm{N}$ and $\mathrm{CO}_{2}$ (exogenous variables) on soil water content, shoot biomass productivity, shoot litter, microbial biomass and diversity of soil microarthropods and nemato des (endogenous variables; white rectangle). The model fit the data well: $\chi^{2} 15=6.93, P=0.96$. Numbers on arrows are unstandardized path coefficients. Width of the arrows indicated the strength of the causal influence: green or red, bold arrows indicate significant relationships $(P<0.05)$, green or red arrows indicate marginally significant relationships $(0.05<P<0.1)$, black, single-headed arrows indicate non-significant relationships $(P$ $>0.1)$. Green arrows indicate significant and marginally significant positive relationships and red ar rows significant and marginally significant negative relationships. Circles indicate error terms (e1-e7); doubleheaded errors indicate correlations between error terms.

\section{Discussion}

Our hypothesis (1) predicting $e \mathrm{CO}_{2}$ to increase the abundance and biodiversity of soil biota, was confirmed in part as $e \mathrm{CO}_{2}$ increased the density and taxa richness of about half of the groups of soil biota. Elevated $\mathrm{CO}_{2}$ increased soil water content most likely by decreasing leaf stomatal conductance (Lee et al. 2011). Elevated $\mathrm{CO}_{2}$ also increased plant shoot and root biomass as shown before at the same field site (Reich et al. 2001a, 2006). Increased shoot biomass led to more aboveground shoot litter material. Soil water content and root biomass, 
Table S1 | Results of structural equation models of global cha nge effects on soil biota illustrated in Fig. 3a an d 3b. Given are the unstandardized path coefficients (estimates), standard error of regression weight (S.E.), the critical value for regression weight (C.R.; $z$ = estimate / S.E.) and level of significance for regression weight $(P)$. For more information of exogenous and endogenous va riables as well as on model fit see main text.

\begin{tabular}{|c|c|c|c|c|c|c|}
\hline a) & & & Estimate & S.E. & C.R. & $P$ \\
\hline Root biomass & $\leftarrow$ & $\mathrm{CO}_{2}$ & 91.65 & 65.15 & 1.41 & 0.160 \\
\hline Soil water & $\leftarrow$ & $\mathrm{CO}_{2}$ & 0.06 & 0.02 & 3.04 & 0.002 \\
\hline Soil water & $\leftarrow$ & Root biomass & 0.00 & 0.00 & 1.17 & 0.243 \\
\hline Microbial biomass & $\leftarrow$ & $\mathrm{CO}_{2}$ & 0.09 & 0.04 & 2.29 & 0.022 \\
\hline Microbial biomass & $\leftarrow$ & Soil water & 0.37 & 0.28 & 1.34 & 0.180 \\
\hline Ciliates & $\leftarrow$ & Microbial biomass & 0.88 & 0.63 & 1.40 & 0.163 \\
\hline Ciliates & $\leftarrow$ & $\mathrm{CO}_{2}$ & 0.29 & 0.17 & 1.70 & 0.088 \\
\hline Microarthropod & $\leftarrow$ & Ciliates & 0.13 & 0.10 & 1.28 & 0.202 \\
\hline Microarthropod & $\leftarrow$ & $\mathrm{CO}_{2}$ & 0.31 & 0.12 & 2.64 & 0.008 \\
\hline Microarthropod & $\leftarrow$ & Root biomass & 0.00 & 0.00 & 1.03 & 0.303 \\
\hline Gamasid mites & $\leftarrow$ & Microarthropod & 0.26 & 0.09 & 3.05 & 0.002 \\
\hline Amoebae & $\leftarrow$ & Microbial biomass & 0.66 & 0.38 & 1.77 & 0.077 \\
\hline Gamasid mites & $\leftarrow$ & Root biomass & 0.00 & 0.00 & -1.22 & 0.223 \\
\hline Amoebae & $\leftarrow$ & Ciliates & 0.14 & 0.09 & 1.65 & 0.099 \\
\hline b) & & & Estimate & S.E. & C.R. & $P$ \\
\hline Soil water & $\leftarrow$ & $\mathrm{CO}_{2}$ & 0.06 & 0.02 & 3.30 & $<<.001$ \\
\hline Shoot biomass & $\leftarrow$ & $\mathrm{CO}_{2}$ & 100.28 & 50.93 & 1.97 & 0.049 \\
\hline Microbial biomass & $\leftarrow$ & $\mathrm{CO}_{2}$ & 0.09 & 0.04 & 2.30 & 0.021 \\
\hline Shoot litter & $\leftarrow$ & Shoot biomass & 0.20 & 0.09 & 2.26 & 0.024 \\
\hline Microbial biomass & $\leftarrow$ & Soil water & 0.40 & 0.27 & 1.46 & 0.145 \\
\hline Microbial biomass & $\leftarrow$ & $\mathrm{N}$ & -0.05 & 0.03 & -1.47 & 0.141 \\
\hline Microarthropod diversity & $\leftarrow$ & Microbial biomass & 0.19 & 0.18 & 1.04 & 0.301 \\
\hline Microarthropod diversity & $\leftarrow$ & Shoot litter & 0.00 & 0.00 & 2.20 & 0.028 \\
\hline Nematode diversity & $\leftarrow$ & $\mathrm{N}$ & -0.02 & 0.02 & -1.12 & 0.262 \\
\hline Microarthropod diversity & $\leftarrow$ & $\mathbf{N}$ & -0.10 & 0.04 & -2.25 & 0.024 \\
\hline Microarthropod diversity & $\leftarrow$ & $\mathrm{CO}_{2}$ & 0.13 & 0.05 & 2.69 & 0.007 \\
\hline Nematode diversity & $\leftarrow$ & Shoot litter & 0.00 & 0.00 & -1.95 & 0.051 \\
\hline Microarthropod diversity & $\leftarrow$ & Soil water & -0.63 & 0.35 & -1.80 & 0.072 \\
\hline
\end{tabular}

though not significant, remained in the final SEM m odel for the soil food web, whereas shoot litter stayed in the final model for soil biodivers ity, indicating that soil biota slightly benefited from those indirect $\mathrm{CO}_{2}$ effects. By contrast, our proxy measure for quality of plant inputs did not remain in the final SEM models. In addition to the measured pathways, direct pathways from $\mathrm{CO}_{2}$ to some response variables remained in the final models and often were the most significant ones. We suggest that these direct connections represent effects of elevated rhizodeposition (Lamb et al. 2011) at e $\mathrm{CO}_{2}$ (Jones et al. 2009; de Graaff et al. 2010) increasing microbial biomass, the density of ciliates, microarthropod detritivores and microarthropod 
taxa richness (Fig. 3). This is in line with prior findings in this experiment of Chung et al. (2007), indicating that increased microbial biomass at $e \mathrm{CO}_{2}, \mathrm{eN}$ and high plant diversity were not only due to increased plant biomass and of Adair et al. $(2009,2011)$ who reported higher carbon inputs at $e \mathrm{CO}_{2}$ and associated heightened soil microbial activity (as evidenced by increased soil $\mathrm{CO}_{2}$ flux). Moreover, Dijkstra et al. (2005) reported that $e \mathrm{CO}_{2}$ increased labile soil $\mathrm{C}$ at the same field site, potentially representing elevated root exudation (Hungate et al. 1997; Box et al. 1998). However, more studies are needed verifying this proposed indirect pathway by directly measuring root rhizodeposition and accompa nying effects on soil biota in response to $e \mathrm{CO}_{2}$ as the present study indicates its prime importance in mediating global change effects below ground.

Similar to a recent study on plant diversity effects on soil biota (Scherber et al. 2010), we found some evidence for the bottom-up control of belowground food webs: increased rhizodeposition and thus resource availability led to elevated microbial biomass and density of ciliates and microarthropod detritivores, which increased the density of amoebae and gamasid mites (predators). The positive effect of $e \mathrm{CO}_{2}$ on soil microorganisms (e.g., Rice et al. 1994; Hu et al. 2001; Carney et al. 2007) and soil animals (e.g., Yeates \& Newton 2009; Blankinship et al. 2011) is well established; however, the present study suggests that $e \mathrm{CO}_{2}$ influences soil biota mainly through increased rhiz odeposition, and that these effects are, in contrast to the conclusions of the meta-analysis by Blankinship et al. (2011), still significant for several groups of soil biota after 13 years of $\mathrm{CO}_{2}$ manipulation. The pronounced response of soil biota to $e \mathrm{CO}_{2}$ - in comparison to the other global change agents - may be due to the deficiency of soil organic matter in the sandy outwash (Reich et al. 2001a). This is likely to result in shifts of soil biotic composition and fun ctioning (Drigo et al. 2010).

In contrast to hypothesis (2) expecting positive $\mathrm{N}$ addition effects on soil biota, the number of nematode predators, microarthropod herbivores (only marginally significant) and the taxa richness of nematodes and microarthropods decreased significantly at eN (Figs. 2b, 3). Moreover, ciliate density was minimal at $e \mathrm{~N}$ in the drought treatment, whereas fungal feeding nematodes as well as astigmatic and prostigmatic mites were increased at $\mathrm{eN}$ in comparison to $a \mathrm{~N}$ when $\mathrm{CO}_{2}$ was at ambient levels. The modest and mostly detrimental effect of $e \mathrm{~N}$, in particular on soil biodiversity, is surprising since soil biota are essentially fueled by the energy derived from plants (Högberg et al. 2010), and since $\mathrm{N}$ addition has been shown to increase 
plant productivity (e.g. Reich et al. 2001a, 2006). For instance, van der Wal et al. (2009) found increased diversity of nematodes, collembolans, mites and enchytraeids in a long-term (>40 years) $\mathrm{N}$ fertilization experiment in grassland, attributing this finding to elevated plant productivity. However, previous findings are inconsistent, reporting positive (e.g., Sjursen et al. 2005; van der Wal et al. 2009; Cusack et al. 2011) and negative effects (e.g., Kopeszki 1993; van Diepen et al. 2010; Högberg et al. 2010) on soil biota in various ecosystems. Our results on elevated abundances of fungal feeding nematodes at $\mathrm{eN}$ are in line with the studies of Ruess et al. (1999) and Li et al. (2007), reporting shifts in nematode community structure in response to $\mathrm{N}$ addition in favor of fungal feeding nematodes. This increase in fungal feeding nematodes in the study of Ruess et al. 1999) was associated with an increase in soil fungal biomass due to fertilization.

Our SEM results on soil biodiversity suggest that detrimental $\mathrm{eN}$ effects may have been mainly due to changes in rhizodeposition. Indeed, Högberg et al. (2010) reported reduced belowground $\mathrm{C}$ allocation due to $\mathrm{N}$ addition in a Pinus sylvestris forest, resulting in decreased microbial biomass markers. Moreover, Dijkstra et al. (2005) reported reduced labile soil C at $e N$ at the same field site. Similarly, in BioCON Adair et al. (2009) found that eN stimulates little increase in belowground activities beyond those strictly associated with root biomass, and in fact a decreased total belowground carbon allocation once roots are accounted for. Thus, similar to part of the positive $e \mathrm{CO}_{2}$ effects on soil biota, negative $\mathrm{eN}$ effects may also be due to changes in rhizodeposition. In addition, $\mathrm{N}$ addit ion can decelerate decomposition of soil organic material, including in BioCON (Dijkstra et al. 2004), and change soil community composition and reduce soil biodiversity (Ruess et al. 1999; Frey et al. 2004; Treseder 2008), possibly by favouring few opportunistic taxa, such as shown for $e \mathrm{CO}_{2}$ effects on microbial communities (Drigo et al. 2010).

In contrast to hypothesis (3) expecting summer drought to decrease the abundance and biodiversity of soil biota, summer drought only had few effects on soil biota; only the density of microarthropod herbivores decreased in response to reduced precipitation and the density of ciliates at $\mathrm{eN}$ and reduced precipitation. This is surprising given the strong dependency of many taxa of soil biota on water availability (Cole man et al. 2004) and the significant reduction of summer precipitation by approximately $-45 \%$. Many global change experiments found detrimental drought effects on soil biota (e.g., Lindberg \& Bengtsson 2005; Landesman et al. 
2011; Kardol et al. 2011; Blankinship et al. 2011) and Blankinship et al. (2011) found precipitation to have significantly stronger effects on soil biota than $e \mathrm{CO}_{2}$ and warming. In addition, the positive impact of precipitation inte nsified with time (Blankinship et al. 2011), a change that should have been covered by our study examining drought effects after four years. However, soil biota colonizing the upper $10 \mathrm{~cm}$ of the soil may also be adapted to drought events as the soil is a sandy outwash (Reich et al. 2001a), which dries out quickly during summer. Further studies are needed to investigate the generality of our findings as well as long-term effects of summer drought.

Recent reviews on global change effects on soil biota concluded that there is an urgent need for long-term studies investigating interactions between different agents acting in concert (Maraldo \& Holmstrup 2010; Blankinship et al. 2011). Indeed, effects of global change agents depended on each other. Although we found no significant three-way interactions between $\mathrm{CO}_{2}, \mathrm{~N}$ and summer drought, there were several two-way interactions, supporting our hypothesis (4) in part, expecting significant inter active effects of global change agents on the abundance and biodiversity of soil biota. Remarkably, five of the six two-way interactions were those between $\mathrm{CO}_{2}$ and $\mathrm{N}$. Although the direction of interactions varied between groups of soil biota, the response of soil microarthropod taxa richness is of specific interest as it is in line with a recent study on plant diversity responses (Reich 2009). Soil microarthropod taxa richness decreased significantly at $e \mathrm{~N}$, but only at $a \mathrm{CO}_{2}$ (Fig. 2b), suggesting that $e \mathrm{CO}_{2}$ ameliorated the negative effect of $\mathrm{N}$ enrichment. Reich (2009) found the same interaction for plant species richness and ascribed the changes to multiple effects of $\mathrm{CO}_{2}$ and $\mathrm{N}$ on plant traits and soil resources altering competitive interactions between plant species. Impacts on soil microarthropod taxa richness in the present study were not due to changes in plant species richness $(r=0.14, P=0.35)$. The reduction in rhizodeposition at $e \mathrm{~N}$ suggested above may have been counteracted by $e \mathrm{CO}_{2}$ through elevated shoot litter inputs, increase rhiz odeposition and soil microbial biomass.

In contrast to microarthropod taxa richness, nematode taxa richness was at minimum at $e \mathrm{CO}_{2}$ and $\mathrm{eN}$ (Fig. 3b), indicating more adverse belowground consequences for biodiversity of interactions between these global change agents. In accordance with the response of microarthropod taxa richness, there was a slight negative effect of $e \mathrm{~N}$ on nematode taxa richness, suggesting decreased rhizodeposition (Fig. 3b). This assumption is supported by the 
finding that the density of predatory nematodes strongly decreased at $e \mathrm{~N}$. As predatory nematodes indicate complex and diverse soil food we bs (Ferris et al. 2001; Williamson et al. 2005; Eisenhauer et al. 2011), we suggest that $\mathrm{N}$ enrichment leads to simplified belowground food webs.

Elevated $\mathrm{CO}_{2}$ had an indirect negative effect on nematode species richness through increasing shoot litter biomass. This effect may have been due to changes in nematode community composition due to the promotion of few dominant ne matode and/or microarthropod species. Moreover, $e \mathrm{CO}_{2}$ can change plant stoichiometry (e.g., Novotny et al. 2007; Reich 2009) and high excess $C$ has been shown to fuel plant defense compounds, reducing the palatability of plant tissue (Agrawal \& Fishbein 2006). This assumption is supported by the significant interactive effect of $\mathrm{CO}_{2}$ and $\mathrm{N}$ on microarthropod herbivores: densities were markedly higher in the ambient treatment than in the elevated treatments. The reasons for the differential response of nematode taxa richness and microarthropod taxa richness to litter biomass remain unclear, but perhaps are driven by complex interactions between bottom-up and top-down forces (Hoeksema et al. 2000), which cannot be comprehensively evaluated without detailed knowledge of all possible food sou rces and trophic interactions. For instance, both Astigmata and Prostigmata as well as fungal feeding nematodes had higher densities at $e \mathrm{~N}$ in comparison to $a \mathrm{~N}$ at $a \mathrm{CO}_{2}$, whereas the opposite was true at $e \mathrm{CO}_{2}$. This indicates distinct shifts in microbial community composition and in the ratio between bacteria and fungi in response to global change agents (Ruess et al. 1999; Chung et al. 2007), which were not reflected by total microbial biomass. Future studies should investigate the relative importance of the bacterial and fungal energy channels in varying global change treatments, since shifts in soil microbial community composition are likely to have marked effects on ecosystem functioning (Coleman 1983; Hunt et al. 1987).

In addition to interactions between $\mathrm{CO}_{2}$ and $\mathrm{N}$, the abundance of ciliates varied significantly in response to $\mathrm{eN}$ and summer drought, supporting that the abundance of active ciliates is particularly sensitive to drought events (Foissner 1997). Minimum densities at $\mathrm{eN}$ in the summer drought treatment indicate that the assumed reduction in rhizodeposition due to $\mathrm{N}$ addition was most detrimental under dry conditions. More long-term studies are needed to investigate if this significant interaction will al so materialize in other groups of soil biota, since 
ciliates represent a crucial link between bacteria and higher trophic levels in soil (Ekelund \& Rønn 1994).

Overall, the modest response of soil biota found in the present study may indicate that soil food webs are buffered against global change stressors and/or have already adapted to the treatments. It should however be noted that bottom-up and top-down stressor effects on soil food webs are likely to depend on climatic conditions; thus, there is an urgent need for repeated belowground measurements in long-term global change experiments in order to appreciate the significance of stressor effects in multiple years and varying climatic conditions (Yeates et al. 2003; Li et al. 2007).

In conclusion, four years of summer drought exerted surprisingly minor effects, but long-term (13 years) changes in $\mathrm{CO}_{2}$ and $\mathrm{N}$ availability resulted in modest alterations of soil biotic food webs and biodiversity via several mechanisms, encompassing soil water availability, plant productivity and - most importantly - rhizodeposition. Enrichment of atmospheric $\mathrm{CO}_{2}$ concentrations and elevated $\mathrm{N}$ deposition may result in taxonomically and functionally altered, potentially simplified, soil communities. The overall reduction of soil biodiversity by $11 \%$ at $e \mathrm{~N}\left(F_{1,36}=8.58, P=0.006\right)$ likely is an underestimation of real consequences as many taxa have not been identified to species level. Simplification of soil food webs due to $\mathrm{N}$ deposition may thus be similar to or even more pronounced than that of plant communities (Clark \& Tilman 2008; Reich 2009), and of particular concern as soils house a considerable fraction of global biodiversity (Decaëns 2010).

\section{Acknowledgements}

We thank Molly Heit for help during field sampling, and Cindy Buschena, Montara Roberts, Chris Bergquist and Susan Barrott (University of Minnesota) for their help during the processing of soil samples, and Michael Bonkowski (University of Cologne) for his helpful comments on the manuscript. Constructive comments of two anonymous reviewers improved the manuscript considerably. Nico Eisenhauer gratefully acknowledges funding by the Deutsche Forschungsgemeinschaft (DFG; Ei 862/1-1). This work was supported by the Department of Energy (DOE/DE-FG02-96ER62291) and the National Science Foundation (NSF Biocomplexity 0322057, NSF LTER DEB 9411972 (1994-2000), DEB 0080382 (2000-2006), and 
DEB 0620652 (2006-2012), and NSF LTREB 0716587). Further, this research was supported by the Department of Energy, Office of Science (BER) through the Midwestern Regional Center of the National Institute for Climatic Change Research at Michigan Technological University (DE-FC02-06ER64158).

\section{References}

Adair, E. C., Reich, P. B., Hobbie, S. E. \& Knops, J. M. H., 2009. Interactive effects of time, $\mathrm{CO}_{2}$, $\mathrm{N}$, and diversity on total belowground carbon alloca tion and ecosystem carbon storage in a grassland community. Ecosystems 12, 1037-1052.

Adair, E. C., Reich, P. B., Trost, J. J. \& Hobbie, S. E., 2011. Elevated $\mathrm{CO}_{2}$ stimulates grassland soil respiration by increasing carbon inputs rather than by enhancing soil moisture. Global Change Biology 17, 3546-3563.

Agrawal, A. A. \& Fishbein, M., 2006. Plant defense syndromes. Ecology 87, S132-S149.

Anderson, J. P. E. \& Domsch, K. H., 1978. A physiological method for the quantitative measurement of microbial biomass in soils. Soil Bio logy and Biochemistry 10, 215-221.

Arbuckle, J. L., 2010. IBM SPSS Amos 19 User's Guide. Amos Development Corporation, Crawfordville, FL, USA.

Assessment Millenium Ecosystem (MA), 2005. Ecosystems and human well-being: Biodiversity Synthesis. World Resources Institute, Washington DC, US.

Bardgett, R. D. \& Wardle, D. A., 2010. Aboveground-Belowground Linkages, Biotic Interactions, Ecosystem Processes, and Global Change. Oxford Series in Ecology and Evolution, Oxford University Press, New York, USA.

Beck, T., Joergensen, R. G., Kandeler, E., Makeschin, E., Nuss, E., Oberholzer, H. R. \& Scheu, S., 1997. An inter-laboratory comparison of ten different ways of measuring soil microbial biomass C. Soil Biology and Biochemistry 29, 1023-1032.

Blagodatskaya, E., Blagodatsky, S., Dorodnikov, M. \& Kuzyakov, Y., 2010. Elevated atmospheric $\mathrm{CO}_{2}$ increases microbial growth rates in soil: results of three $\mathrm{CO}_{2}$ enrichment experiments. Global Change Biology 16, 836-848.

Blankinship, J. C., Niklaus, P. A. \& Hungate, B. A., 2011. A meta-analysis of responses of soil biota to global change. Oecologia 165, 553-65.

Box, P. O., Cheng, W. \& Johnson, D. W., 1998. Elevated $\mathrm{CO}_{2}$, rhizosphere processes, and soil organic matter decomposition. Plant and Soil 202, 167-174. 
Butenschoen, O., Scheu, S. \& Eisenhauer, N., 2011. Interactive effects of warming, soil humidity and plant diversity on litter decomposition and microbial activity. Soil Biology and Biochemistry 43, 1902-1907.

Carney, K. M., Hungate, B. A., Drake, B. G. \& Megonigal, J. P., 2007. Altered soil microbial community at elevated $\mathrm{CO}_{2}$ leads to loss of soil carbon. Proceedings of the National Academy of Sciences of the United States of America 104, 4990-5.

Chung, H., Zak, D. R., Reich, P. B. \& Ellsworth, D. S., 2007. Plant species richness, elevated $\mathrm{CO}_{2}$, and atmospheric nitrogen deposition alter soil microbial community composition and function. Global Change Biology 13, 980-989.

Clark, C. M. \& Tilman, D., 2008. Loss of plant species after chronic low-level nitrogen deposition to prairie grasslands. Nature 451, 712-5.

Coleman, D. C., 1983. Biological strategies of nutrient cycling in soil systems. Advances in Ecological Research 13, 1-55.

Coleman, D. C., Crossley, J. D. A. \& Hendrix, P. F., 2004. Fundamentals of Soil Ecology. Elsevier Academic Press, San Diego, USA.

Cusack, D. F., Silver, W. L., Torn, M. S., Burton, S. D. \& Firestone, M. K., 2011. Changes in microbial community characteristics and soil organic matter with nitrogen additions in two tropical forests. Ecology 92, 621-632.

Darbyshire, J. F., Wheatley, R., Greaves, M. \& Inkson, R., 1974. A rapid micromethod for estimating bacterial and Protozoan populations in soil. Revue d'Ecologie et Biologie du Sol 11, 465-475.

DeForest, J. L., Zak, D. R., Pregitzer, K. S. \& Burton, A. J., 2004. Atmospheric nitrate deposition, microbial community composition, and enzyme activity in northern hardwood forests. Soil Science Society of America Journal 68, 132-138.

Decaëns, T., 2010. Macroecological patterns in soil communities. Global Ecology and Biogeography 19, 287-302.

de Graaff, M.-A., Classen, A. T., Castro, H. F. \& Schadt, C. W., 2010. Labile soil carbon inputs mediate the soil microbial community composition and plant residue decomposition rates. New Phytologist 188, 1055-1064.

Dijkstra, F. A., Hobbie, S. E., Knops, J. M. H. \& Reich, P. B., 2004. Nitrogen deposition and plant species interact to influence soil carbon stabiliza tion. Ecology Letters 7, 1192-1198.

Dijkstra, F. A., Hobbie, S. E., Reich, P. B. \& Knops, J. M. H., 2005. Divergent effects of elevated $\mathrm{CO}_{2}, \mathrm{~N}$ fertilization, and plant diversity on soil $\mathrm{C}$ and $\mathrm{N}$ dynamics in a grassland field experiment. Plant and Soil 272: 41-52.

Drigo, B., Pijl, A. S., Duyts, H., Kielak, A. M., Gamper, H. A., Houtekamer, M. J., Boschker, H. T. S., Bodelier, P. L. E., Whiteley, A. S., van Veen, J. A. \& Kowalchuk, G. A., 2010. Shifting 
carbon flow from roots into associated microbial communities in response to elevated atmospheric $\mathrm{CO}_{2}$. PNAS 107: 10938-10942.

Dukes, J. S., Chiariello, N. R., Cleland, E. E., Moore, L. A, Shaw, M. R., Thayer, S., Tobeck, T., Mooney, H. A \& Field, C. B., 2005. Responses of grassland production to single and multiple global environmental changes. PLoS biology 3: e319.

Eisenhauer, N., Migunova, V. D., Ackermann, M., Ruess, L. \& Scheu, S., 2011. Changes in plant species richness induce functional shifts in soil $n$ ematode communities in experimental grassland. PloS one 6: e24087.

Ekelund, F. \& Rønn, R., 1994. Notes on protozoa in agricultural soils with emphasis on heterotrophic flagellates and naked amoebae and their ecology. FEMS Microbiology Reviews 15, 321-353.

Ferris, H., Bongers, T. \& de Goede, R. G. M., 2001. A framework for soil food web diagnostics: extension of the nematode faunal analysis concept. Applied Soil Ecology 18, 13-29.

Field, C. B., Jackson, R. B. \& Mooney, H. A., 1995. Stomatal responses to increased $\mathrm{CO}_{2}$ : implications from the plant to the global scale. Plant, Cell and Environment 18, 12141225.

Fjellberg, A., 1980. Identification keys to Norwegian Collembola. Norsk Entomologisk Forening, Ås.

Foissner, W., 1997. Global soil ciliate (Protozoa, Ciliophora) diversity: a probability-based approach using large sample collections from Africa, Australia and Antarctica. Biodiversity and Conservation 6, 1627-1638.

Frey, S. D., Knorr, M., Parrent, J. L. \& Simpson, R. T., 2004. Chronic nitrogen enrichment affects the structure and function of the soil microbial community in temperate hardwood and pine forests. Forest Ecology and Management 196, 159-171.

Gisin, H., 1960. Collembolenfauna Europas. Museum d'Histoire Naturelle, Geneve, Switzerland.

Grace, J. B., 2006. Structural equation modeling and natural systems. Cambridge University Press, Cambridge, UK.

Hendrey, G. R., Lewein, K. F. \& Nagy, J., 1993. Free air carbon dioxide enrichment: development, progress, results. Vegetatio 104-105, 17-31.

Hoeksema, J. D., Lussenhop, J. \& Teeri, J. A., 2000. Soil nematodes indicate food web responses to elevated atmospheric $\mathrm{CO}_{2}$. Pedobiologia 735, 725-735.

Hopkin, S. P., 2007. A key to the springtails (Collembola) of Britain and Ireland. Field Studies Council (AIDGAP Project). 
Hu, S., Chapin, F. S., Firestone, M. K., Field, C. B. \& Chiariello, N. R., 2001. Nitrogen limitation of microbial decomposition in a grassland under ele vated $\mathrm{CO}_{2}$. Nature $409,188-191$.

Hungate, B. a, Dukes, J. S., Shaw, M. R., Luo, Y. \& Field, C. B., 2003. Nitrogen and climate change. Science 302, 1512-1513.

Hungate, B. A., Holland, E. A., Jackson, R. B., lii, F. S. C., Mooneyk, H. A. \& Field, C. B., 1997. The fate of carbon in grasslands under carbon dioxi de enrichment. Nature 388, 576-580.

Hunt, H. W., Coleman, D. C., Ingham, E. R., Ingham, R. E., Elliott, E. T., Moore, J. C., Rose, S. L., Reid, C. P. P. \& Morley, C. R. I., 1987. The detrital food web in a shortgrass prairie. Biology and Fertility of Soils, 57-68.

Hurley, M. \& Roscoe, M., 1983. Automated statistical analysis of microbial enumeration by dilution series. Journal of Applied Bacteriology 55, 159-164.

Högberg, M. N., Briones, M. J. I., Keel, S. G., Metcalfe, D. B., Campbell, C., Midwood, A. J., Thornton, B., Hurry, V., Linder, S., Näsholm, T. \& Högberg, P., 2010. Quantification of effects of season and nitrogen supply on tree below-ground carbon transfer to ectomycorrhizal fungi and other soil organisms in a boreal pine forest. New Phytologist 187, 485-93.

IPCC, 2007. Climate Change 2007: The Physical Science Basis. Contribution of Working Group I to the Fourth Assessment Report of the Intergovernmental Panel on Climate Change (S. Solomon, D. Qin, M. Manning, Z. Chen, M. Marquis, K.B. Averyt, M. Tignor, and H.L. Miller, eds.). Cambridge University Press, Cambridge, United Kingdom and New York, NY, USA.

Jones, D. L., Nguyen, C. \& Finlay, R. D., 2009. Carbon flow in the rhizosphere: carbon trading at the soil-root interface. Plant and Soil 321, 5-33.

Kardol, P., Cregger, M. A., Campany, C. E. \& Classen, A. T., 2010. Soil ecosystem functioning under climate change: plant species and community effects. Ecology 91, 767-781.

Kardol, P., Reynolds, W. N., Norby, R. J. \& Classen, A. T., 2011. Climate change effects on soil microarthropod abundance and community structure. Applied Soil Ecology 47, 37-44.

Kempson, D., Lloyd, M. \& Ghelardi, R., 1963. A new extractor for woodland litter. Pedobiologia 3, 1-21.

Kerr, R. A., 2009. Global warming is changing the world. Science 316, 188-190.

Kopeszki, H., 1993. Effects of fertilization on the mesofauna, especially Collembolan, in different forest habitats in the Bohemian woods. Zo ologischer Anzeiger 231, 83-98.

Krantz, G. W. \& Walter, D. E., 2009. A manual of Acarology. Texas Tech University Press, Lubbock, Texas, USA. 
Lamb, E. G., Kennedy, N. \& Siciliano, S. D., 2011. Effects of plant species richness and evenness on soil microbial diversity and function. Plant and Soil 338, 483-495.

Landesman, W. J., Treonis, A. M. \& Dighton, J., 2011. Effects of a one-year rainfall manipulation on soil nematode abundances and community compositi on. Pedobiologia 54, 87-91.

Lee, T. D., Barrott, S. H. \& Reich, P. B., 2011. Photosynthetic responses of 13 grassland species across 11 years of free-air $\mathrm{CO}_{2}$ enrichment is modest, consistent and independent of $\mathrm{N}$ supply. Global Change Biology 17, 2893-2904.

Li, Q., Liang, W., Jiang, Y., Shi, Y., Zhu, J. \& Neher, D. A., 2007. Effect of elevated $\mathrm{CO}_{2}$ and $\mathrm{N}$ fertilisation on soil nematode abundance and diversity in a wheat field. Applied Soil Ecology 36, 63-69.

Lindberg, N. \& Bengtsson, J., 2005. Population responses of oribatid mites and collembolans after drought. Applied Soil Ecology 28, 163-174.

Luo, Y., Gerten, D., Le Maire, G., Parton, W. J., Weng, E., Zhou, X., Keough, C., Beier, C., Ciais, P., Cramer, W., Dukes, J. S., Emmett, B., Hanson, P. J., Knapp, A., Linder, S., Nepstad, D. \& Rustad, L., 2008. Modeled interactive effects of precipitation, temperature, and $\mathrm{CO}_{2}$ on ecosystem carbon and water dynamics in different climatic zones. Global Change Biology 14, 1986-1999.

Maraldo, K. \& Holmstrup, M., 2010. Enchytraeids in a changing climate: a mini-review. Pedobiologia 53, 161-167.

Moran, M. D., 2003. Arguments for rejecting the sequential Bonferroni in ecological. OIKOS $100,403-405$.

Niklaus, P. A. \& Körner, C., 1996. Responses of soil microbiota of a late successional alpine grassland to long term $\mathrm{CO}_{2}$ enrichment. Plant and Soil 184, 219-229.

Novotny, A. M., Schade, J. D., Hobbie, S. E., Kay, A. D., Kyle, M., Reich, P. B. \& Elser, J. J., 2007. Stoichiometric response of nitrogen-fixing and non-fixing dicots to manipulations of $\mathrm{CO}_{2}$, nitrogen, and diversity. Oecologia 151, 687-696.

Reich, P. B., 2009. Elevated $\mathrm{CO}_{2}$ reduces losses of plant diversity caused by nitrogen deposition. Science 326, 1399-1402.

Reich, P. B., Hobbie, S. E., Lee, T., Ellsworth, D. S., West, J. B., Tilman, D., Knops, J. M. H., Naeem, S. \& Trost, J., 2006. Nitrogen limitation constrains sustainability of ecosystem response to $\mathrm{CO}_{2}$. Nature 440, 922-925.

Reich, P. B., Knops, J., Tilman, D., Craine, J., Ellsworth, D., Tjoelker, M., Lee, T., Wedin, D., Naeem, S., Bahauddin, D., Hendrey, G., Jose, S., Wrage, K., Goth, J. \& Bengston, W., 2001a. Plant diversity enhances ecosystem responses to elevated $\mathrm{CO}_{2}$ and nitrogen deposition. Nature 411, 809-812. 
Reich, P. B., Peterson, D. W., Wedin, D. A. \& Wrage, K., 2001b. Fire and vegetation effects on productivity and nitrogen cycling across a forest. Ecology 82, 1703-1719.

Rice, C. W., Garcia, F. O., Hampton, C. O. \& Owensby, C. E., 1994. Soil microbial response in tallgrass prairie to elevated $\mathrm{CO}_{2}$. Plant and Soil 165, 67-74.

Ruess, L., 1995. Studies on the nematode fauna of a $n$ acid forest soil: spatial disturbance and extraction. Nematologica 41, 229-239.

Ruess, L., Michelsen, A., Schmidt, I. K. \& Jonasson, S., 1999. Simulated climate change affecting microorganisms, nematode density and biodiversity in subarctic soils. Plant and Soil 212, 63-73.

Sala, O. E., 2000. Global Biodiversity Scenarios for the Year 2100. Science 287, 1770-1774.

Schaefer, M., 2000. Brohmer - Fauna von Deutschland. Quelle \& Meyer, Wiebelsheim, Germany.

Scherber, C., Eisenhauer, N., Weisser, W. W., Schmid, B., Voigt, W., Fischer, M., Schulze, E.-D., Roscher, C., Weigelt, A., Allan, E., Bessler, H., Bonkowski, M., Buchmann, N., Buscot, F., Clement, L. W., Ebeling, A., Engels, C., Halle, S., Kertscher, I., Klein, A.-M., Koller, R., König, S., Kowalski, E., Kummer, V., Kuu, A., Lange, M., Lauterbach, D., Middelhoff, C., Migunova, V. D., Milcu, A., Müller, R., Partsch, S., Petermann, J. S., Renker, C., Rottstock, T., Sabais, A., Scheu, S., Schumacher, J., Temperton, V. M. \& Tscharntke, T., 2010. Bottom-up effects of plant diversity on multitrophic interactions in a biodiversity experiment. Nature 468, 553-556.

Scheu, S., 1992. Automated measurement of the respiratory response of soil microcompartments: active microbial biomass in earthworm faces. Soil Biology and Biochemistry 24, 1113-1118.

Sjursen, H., Michelsen, A. \& Jonasson, S., 2005. Effects of long-term soil warming and fertilization on microarthropod abundances in three sub-arctic ecosystems. Applied Soil Ecology 30, 148-161.

Treseder, K. K., 2008. Nitrogen additions and microbial biomass: a meta-analysis of ecosystem studies. Ecology Letters 11, 1111-1120.

van der Wal, A., Geerts, R. H. E. M., Korevaar, H., Schouten, A. J., Jagers op Akkerhuis, G. A. J. M., Rutgers, M. \& Mulder, C., 2009. Dissimilar response of plant and soil biota communities to long-term nutrient addition in grasslands. Biology and Fertility of Soils 45, 663-667.

van Diepen, L. T. A., Lillekov, E. A., Pregitzer, K. S. \& Miller, R. M., 2010. Simulated nitrogen deposition causes a decline of intra- and extraradical abundance of arbuscular mycorrhizal fungi and changes in microbial community structure in northern hardwood forests. Ecosystems 13, 683-695. 
Vitousek, P. M., Mooney, H. A., Lubchenco, J. \& Melillo, J. M., 1997. Human domination of Earth's ecosystems. Science 277, 494-499.

West, J. B., Hobbie, S. E. \& Reich, P. B., 2006. Effects of plant species diversity, atmospheric $\left[\mathrm{CO}_{2}\right]$, and $\mathrm{N}$ addition on gross rates of inorganic $\mathrm{N}$ release from soil organic matter. Global Change Biology 12, 1400-1408.

Williamson, W. M., Wardle, D. A. \& Yeates, G. W., 2005. Changes in soil microbial and nematode communities during ecosystem decline across a long-term chronosequence. Soil Biology and Biochemistry 37, 1289-1301.

Wuebbles, D. J. \& Hayhoe, K., 2004. Climate change projections for the United States Midwest. Mitigation and Adaptation Strategies for Global Cha nge 9, 335-363.

Yeates, G. W. \& Newton, P. C. D., 2009. Long-term changes in topsoil nematode populations in grazed pasture under elevated atmospheric carbon dioxide. Biology and Fertility of Soils 45, 799-808.

Yeates, G. W., Bongers, T., de Goede, R. G. M., Freckman, D. W. \& Georgieva, S. S., 1993. Feeding habits in soil nematode families and genera - an outline for soil ecologist. Journal of Nematology 25, 315-331.

Yeates, G. W., Newton, P. C. D. \& Ross, D. J., 2003. Significant changes in soil microfauna in grazed pasture under elevated carbon dioxide. Biology and Fertility of Soils 38, 319-326.

Zak, D. R., Pregitzer, K. S., King, J. S. \& Holmes, W. E., 2000. Elevated atmospheric $\mathrm{CO}_{2}$, fine roots and the response of soil microorganisms: a review and hypothesis. New Phytologist $147,201-222$. 


\section{Chapter 5}

\section{Changes in soil processes indicated by soil nematodes: \\ Interactive responses to elevated $\mathrm{CO}_{2}$, nitrogen deposition and reduced precipitation}

Simone Cesarz, Peter B. Reich, Stefan Scheu, Liliane Ruess, Matthias Schaefer and Nico Eisenhauer

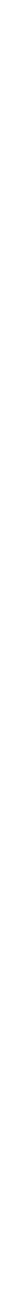




\section{Abstract}

Soils store $\sim 80 \%$ of global terrestrial organic carbon and alterations in fluxes into and out of this pool may interact with ongoing climate change. Little is known about the responses of belowground food webs driving soil $\mathrm{C}$ dynamics to co-occurring global change agents. We investigated grassland at ambient and elevated atmospheric $\mathrm{CO}_{2}$ concentration, ambient and enriched nitrogen input, and ambient and reduced summer precipitation to evaluate how these agents affect soil processes by utilizing nematodes as indicator group of soil food web structure. The density of fungal feeders increased considerably due to $\mathrm{N}$ addition, but this effect was alleviated by elevated $\mathrm{CO}_{2}$. Nematode community analysis suggested that the decomposer community switched from bacterial-dominated to a fungal-dominated system at elevated $\mathrm{N}$, indicating shifts in the microbial community as well as in the functioning of belowground food webs. Reduced densities of root-fe eding Longidoridae at elevated $\mathrm{CO}_{2}$ and elevated $\mathrm{N}$ suggest increased plant performance and lower susceptibility to herbivores. Predacious nematodes were less abundant at elevated $\mathrm{N}$, and changes in nematode community structure suggest reduced top-down forces and simplified soil food webs. At elevated $\mathrm{CO}_{2}$ top-down forces likely were strengthened as the density of bacterial-feeding nematodes did not change despite increased belowground carbon flow. The studied global change agents interactively and differentially affected functional guilds of soil nematodes, suggesting complex changes in soil processes with decomposition processes shifting towards the fungal channel at elevated N. Overall, the results indicate that shifts in soil food web structure may cause distinct changes in ecosystem functioning. 


\section{Introduction}

Human activity leads to changes in atmospheric $\mathrm{CO}_{2}$ concentration, nitrogen $(\mathrm{N})$ deposition and precipitation regimes with considerable impacts on ecosystem functioning (IPCC 2007). These global change agents are acting in concert, and understanding their interactive effects is crucial to predict the consequences for ecosystem functions and services (Reich et al. 2006a). Typically, in terrestrial ecosystems $>90 \%$ of the biomass produced by plants enters the dead organic matter pool forming the basis of the decomposer system (Schlesinger and Andrews 2000). Thus, understanding interactions between plants and decomposers is of high importance, especially as the balance between carbon sequestration and carbon loss depends on those interrelationships (Gessner et al. 2010).

Since the industrial revolution, $\mathrm{CO}_{2}$ concentrations in the atmosphere have increased from approximately 270 ppm to 380 ppm in 2005 and presumably will reach 550 ppm by the year 2050 (IPCC 2007; Rogelj et al. 2012). Higher atmospheric $\mathrm{CO}_{2}$ concentrations significantly impact plant physiological processes. At least three responses are relevant to the decomposer system. Most prominent is the increase in plant carbon (C) acquisition which leads to both greater biomass production (Ainsworth and Long 2005) and greater labile $C$ inputs to the soil (Adair et al. 2011). Also, increasing C acquisition is usually associated with a larger C-to-N ratio of live plant tissue and therefore reduced tissue quality for consumers (Körner 2000). These plant responses have cascading effects on both aboveground (Lau and Tiffin 2009) and belowground consumers (Blankinship et al. 2011), the latter representing important drivers of soil processes, such as organic matter decomposition and nutrient mineralization. Within the decomposer system, microbial biomass is enhanced at elevated atmospheric $\mathrm{CO}_{2}$ concentrations (He et al. 2010) probably due to increased rhizodeposition (Zak et al. 1993; Grayston et al. 1998). Furthermore, arbuscular mycorrhizal fungi (AMF) rapidly respond to 
increased input of root derived resources at elevated atmospheric $\mathrm{CO}_{2}$ concentrations (Drigo et al. 2010), although responses are variable (Wolf et al. 2003; Antoninka et al. 2011). The response of soil animals to elevated $\mathrm{CO}_{2}$ depends on ecosystem type and plant community composition (Blankinship et al. 2011). The recent study of Eisenhauer et al. (2012) proposed bottom-up control of soil fauna due to higher rhizo deposition in a long-term study (13 years) in temperate grassland. Belowground responses to higher plant biomass production and rhizodeposition under elevated atmospheric $\mathrm{CO}_{2}$ can either increase $\mathrm{C}$ loss by increasing bottom-up forces leading to increased decomposition or enhance $C$ sequestration when topdown forces counter bottom-up forces (Wardle et al. 1998; Schulze and Freibauer 2005). This balance has major implications for ecosystem feedback effects to atmospheric $\mathrm{CO}_{2}$ concentrations and therefore on the global $\mathrm{C}$ cycle and may depend on other co-occurring global change agents. However, effects of elevated $\mathrm{CO}_{2}$ concentrations on soil organisms may depend on other environmental conditions, such as N inputs (Hoeksema et al. 2000; Chung et al. 2007).

Nitrogen is a key nutrient in terrestrial ecosystems (LeBauer and Treseder 2008) and crucially determines processes such as decomposition, mineralization and nitrification (Swift et al. 1979; Parton et al. 2007). The previously reported effects of $\mathrm{N}$ addition on different processes are positive, negative or neutral, reflecting that $\mathrm{N}$ regimes in soils are poorly understood and may be context-dependent (Knorr et al. 2005; Keeler et al. 2008). N fertilization often increases plant productivity (Reich et al. 2001c), but also decreases above- and belowground biodiversity by favoring dominant species (Clark and Tilman 2008; Reich 2009; Eisenhauer et al. 2012). Elevated $\mathrm{N}$ availability can decrease rhizodeposition (Dijkstra et al. 2005; Högberg et al. 2010) with neutral to negative effects on soil microorganisms and higher trophic levels of soil organisms (Eisenhauer et al. 2012). As most terrestrial ecosystems are $\mathrm{N}$ limited, 
fertilization and atmospheric $\mathrm{N}$ deposition may indu ce unexpected $\mathrm{CO}_{2}$ responses with poorly understood consequences for the belowground system and ecosystem functioning (Reich et al. 2006b). Previous studies on soil biota found in teractive effects of increased atmospheric $\mathrm{CO}_{2}$ concentrations and $\mathrm{N}$ fertilization (Hoeksema et al. 2000; Hu et al. 2001; Eisenhauer et al. 2012). However, long-term responses of the belowground system remain little understood (Bardgett and Wardle 2010), though they are important as ecosystems often respond slowly to environmental changes (Kuzyakov and Gavrichkova 2010).

In addition to altered $\mathrm{CO}_{2}$ and $\mathrm{N}$ levels, climate is projected to change with altered precipitation regimes (IPCC 2007; Kerr 2007). Soil moisture and related biotic and abiotic parameters are important driving forces for soil processes (Kardol et al. 2010). Drought has mostly negative effects on soil fauna by hampering directly their reproduction and development (Lindberg et al. 2002) or indirectly by changing the composition and biomass of microorganisms (Hawkes et al. 2011) and plants (Kardol et al. 2010). In addition, responses to changes in soil moisture regime depend strongly on the plant community (Gross et al. 2008). Soil moisture may interact with $\mathrm{CO}_{2}$ and $\mathrm{N}$ availability since elevated $\mathrm{CO}_{2}$ levels often increasing soil water content and N content increase with higher soil moisture (Körner 2000; Zhang and Wienhold 2002), therefore complex interactions in soil processes and involved soil biota are likely. However, most previous studies separately investigated the influence of atmospheric $\mathrm{CO}_{2}$ enrichment, $\mathrm{N}$ fertilization and changes in soil water content (Blankinship et al. 2011).

One promising approach to detect changes in soil processes is the investigation of soil food web structure, exemplified by the composition of functional guilds of nematodes (Bongers 1990; Yeates et al. 1993; Ferris et al. 2001). As nematodes have diverse feeding behaviours and life strategies and play a key role in soil food webs, they function as important indicators 
for ecosystem processes (Ferris 2010; Yeates 2010). Functional grouping of nematodes can provide important information to detect changes in soil processes by considering distinct feeding strategies, e.g. bacterial or fungal-feeding, and their responses (tolerance vs. sensitivity) to environmental changes. In addition, nematode based indices allow evaluating ecosystem nutrient status (enriched vs. depleted), structure of the soil food web (complexity vs. simplicity), and the relevance of decomposition channels (bacterial vs. fungal) (Ferris et al. 2001).

We used a well-established global change experiment in grassland (BioCON) to explore interactive effects of elevated atmospheric $\mathrm{CO}_{2}, \mathrm{~N}$ deposition and reduced summer precipitation on soil nematode communities. The Bio CON experiment is ongoing for more than 13 years, and results may therefore not be biased by transient effects caused by the establishment of the experiment. Nematodes comprise diverse trophic groups of different trophic levels; using the approach by Ferris et al. (2001), nematodes can additionally be arranged into functional guilds, i.e., accounting for different life history strategies in each trophic group (Ferris et al. 2001). For instance, opportunistic species are favored by high nutrient supply and are tolerant to environmental stress, whereas sensitive species respond negatively to disturbance. Combining information on different life history strategies and trophic group affiliation allows identification of functional guilds, which respond similarly to food web enrichment and disturbance. Results of Eis enhauer et al. (2012) at the same site indicated altered soil communities mainly due to treatment-induced changes in rhizodeposition (Eisenhauer et al. 2012). The present study goes one step further by arranging nematodes into functional guilds. The calculation of the nematode functional indices introduced above provides information on the functioning of soils communities. 
We hypothesised (1) elevated $\mathrm{CO}_{2}$ to increase the abundance of opportunistic nematod es due to higher resource availability, (2) increased $\mathrm{N}$ fertilization to reduce connectivity and food web complexity, i.e., low number of trophic links and reduced stability of the nematode community, but to increase the abundance of opportunistic nematodes as they may profit from increases in resource availability, (3) reduced precipitation to have non-significant effects on opportunistic soil nematodes as they are adapted to the sandy and dry environment at the study site, and (4) nematode functional guilds to be influenced by interactions of the three global change agents and therefore not to be predictable from single factor effects.

\section{Material and Methods}

\section{Location and experimental design}

The study was carried out within the framework of the BioCON experiment (short for Biodiversity, $\mathrm{CO}_{2}$ and $\mathrm{N}$ ) at Cedar Creek in Minnesota, USA (Reich et al. 2001a; c). The site is situated on an $\mathrm{N}$ limited glacial outwash sand plain with soils containing $94.4 \%$ sand and $2.5 \%$ clay. The climate is continental with warm summers and cold winters. Mean annual precipitation is $660 \mathrm{~mm}$ (Reich et al. 2001a).

The experiment was conducted on secondary successional grassland after removing the previous vegetation. Starting in 1997 , atmospheric $\mathrm{CO}_{2}$ was elevated $\left(e \mathrm{CO}_{2}\right)$ via FACE (Free air $\mathrm{CO}_{2}$ enrichment) technology at $550 \mathrm{ppm}$ in three of six rings $20 \mathrm{~m}$ ID. An ambient $\mathrm{CO}_{2}\left(a \mathrm{CO}_{2}\right)$ concentration (370 ppm) was delivered to the three remaining rings. Within these rings, plots of $2 \times 2 \mathrm{~m}$ were established differing in $\mathrm{N}$ concentration, plant species richness (not considered in the present study) and summer precipitation. Nitrogen was delivered to half of the plots in 
May, June and July as $4 \mathrm{~g} \mathrm{NH}_{4} \mathrm{NO}_{3} \mathrm{~m}^{-2} \mathrm{yr}^{-1}$ slow release ammonium nitrate (ambient $\mathrm{N}=a \mathrm{~N}$, elevated $\mathrm{N}=e \mathrm{~N}$ ). This amount roughly doubles the ambient soil $\mathrm{N}$ availability at this site (Reich et al. 2001b). Since 2007, summer precipitation was reduced on half of the plots ( $r$ PREC; ambient precipitation $=a \mathrm{PREC}$ ) by approximately $45 \%$ with portable rain-out shelters from May to August to simulate summer drought (Eisenhauer et al. 2012) as one additional future global change scenario in this region (Wuebbles and Hayhoe 2004; IPCC 2007).

The main experiment was set up in 1997 with plots differing in plant species richness $(1,4,9$, and 16 species). Here, we focus on 48 randomly chosen 9-species plots now containing 6-7 species on average and belonging to 3-4 functional groups (Reich et al. 2001a; c; Eisenhauer et al. 2012). The sown plant species comprise four species of each of the functional groups C3 grasses, C4 grasses, herbs and $\mathrm{N}$ fixing legumes (Reich et al. 2001a). The experiment was arranged in a complete factorial combination of $2 \times \mathrm{CO}_{2}$ (ambient and $+180 \mathrm{ppm}$ ), $2 \times \mathrm{N}$ (ambient and $+2 \mathrm{~g} \mathrm{NH}_{4} \mathrm{NO}_{3} \mathrm{~m}^{-2} \mathrm{yr}^{-1}$ ) and $2 \times$ summer precipitation (ambient and $-45 \%$ ), replicated six times each.

\section{Sampling}

In August 2010, five weeks after the last $\mathrm{N}$ addition campaign, three soil samples $(2 \mathrm{~cm}$ in diameter, $6 \mathrm{~cm}$ depth) were taken at each plot. Homogenized samples were pooled and stored at $4^{\circ} \mathrm{C}$ until extraction. Nematodes were extracted from the soil samples (10 $\mathrm{g}$ fresh weight) by a modified Baermann method (Ruess 1995). Soil samples were placed in separate plastic vessels with gauze at the bottom coated with a milk filter. The vessels were put in funnels connected to glass vials and watered. Nematodes were left at $20^{\circ} \mathrm{C}$ for $30 \mathrm{~h}$ to traverse the filter. Animals were killed and fixed by formaldehyde solution (4\%). Nematodes were counted and a minimum of 100 individuals, if present, were determined to family or genus level using 
$100 \times$ magnification (Zeiss, Axiovert 135) for arranging them into trophic groups according to Yeates et al. (1993) (Yeates et al. 1993).

\section{Nematode community indices}

To evaluate food web characteristics, Enrichment Index (EI), Structure Index (SI) and Channel Index (Cl) were calculated after Ferris et al. (2001) (Ferris et al. 2001). These three indices are calculated independently from the weighted abundance of nematode guilds. Nematode guilds comprise bacterivores $\left(\mathrm{Ba}_{\mathrm{x}}\right)$, fungivores $\left(\mathrm{Fu}_{\mathrm{x}}\right)$, predators $\left(\mathrm{Pr}_{\mathrm{x}}\right)$ and omnivores $\left(\mathrm{Om}_{\mathrm{x}}\right)$ ranging along the $c-p$ scale from $x=1$ to $x=5$. Nematodes of $c-p 1$ have a short life cycle, high fecundity, are tolerant to disturbance and can be ascribed to $r$-strategists. In contrast, nematodes of $c-p$ 5 produce few large eggs, have a long life cycle combined with a long generation time and are sensitive to disturbance resembling $K$-strategists. Numbers between 1 and 5 reflect gradations between these opposing life history strategies (Neher and Darby 2009). The relative abundance of these guilds reflects certain soil con ditions and characteristics of the soil food web, such as level of enrichment, disturbance and food web complexity. The El provides information about the resource status of the ecosystem investigated. It is based on bacterialfeeding nematodes within $c-p 1\left(\mathrm{Ba}_{1}\right)$ and fungal feeders within $c-p 2\left(\mathrm{Fu}_{2}\right) . \mathrm{Ba}_{1}$ occur in high numbers in disturbed systems, e.g., due to mortality leading to nutrient flushes and high microbial activity (high EI). High availability of complex organic material is reflected in increased densities of $\mathrm{Fu}_{2}$. Therefore, both guilds represent nutrient enriched conditions. In addition, the calculation of the $\mathrm{El}$ is related to nematodes with a wide ecological range $\left(\mathrm{Ba}_{2}\right.$ and also $\mathrm{Fu}_{2}$ ) consequently being ubiquitous. The $\mathrm{El}$ is calculat ed as

$E I=100 \times[e /(e+b)]$ 
with e representing the enrichment component, calculated as the weighted frequencies of $\mathrm{Ba}_{1}$ and $\mathrm{Fu}_{2}$ and $b$ representing the basal food web component, calculated as the weighted frequencies of $\mathrm{Ba}_{2}$ and $\mathrm{Fu}_{2}$.

The Structure Index (SI) combines functional guilds of nematodes of higher $c-p$ values ranging from $c-p$ 3-5. Nematodes within these guilds represent more stable conditions, e.g., by recovering from stress and higher food web connectivity, i.e., a high degree of connectence through many interactions and therefore functional resilience to disturbance (Ferris et al. 2001). High SI values indicate a structured ecosystem with many trophic links, whereas low values stand for simplified and disturbed systems. It is calculated as

$\mathrm{SI}=100 \times[\mathrm{s} /(\mathrm{s}+\mathrm{b})]$,

with s representing the structure food web component calculated as the weighted frequencies of $\mathrm{Ba}_{3}-\mathrm{Ba}_{5}, \mathrm{Fu}_{3}-\mathrm{Fu}_{5}, \mathrm{Pr}_{3}-\mathrm{Pr}_{5}$ and $\mathrm{Om}_{3}-\mathrm{Om}_{5}$ as well as $b$ the basal food web component, calculated as the weighted frequencies of $\mathrm{Ba}_{2}$ and $\mathrm{Fu}_{2}$.

Information about the dominant decomposition channe I is provided by the Channel Index (CI), as it reflects the percentage of fungal-feeding nematodes among the total of fungal feeders and opportunistic bacterial-feeding nematodes. It is calculated as

$\mathrm{Cl}=100 \times\left[0.8 \times \mathrm{Fu}_{2} /\left(3.2 \times \mathrm{Ba}_{1}+0.8 \times \mathrm{Fu}_{2}\right)\right]$,

with $\mathrm{Fu}_{2}$ representing all fungal feeders of $c-p 2$ and $\mathrm{Ba}_{1}$ all bacterial feeders of $c-p 1$ and coefficients represents the population increase rate (Ferris et al. 1996). Low Cl values indicate bacterial dominated decomposition whereas high values refer to a more fungal dominated system. 


\section{Statistical analysis}

Three-way ANOVA (SAS 9.2, SAS Institute Inc., Cary, NC, USA) was used to test for main effects of $\mathrm{CO}_{2}$ (ambient and elevated), $\mathrm{N}$ (ambient and elevated) and precipitation (ambient and reduced) and all possible interactions. Nematode abundance data was log transformed to meet the requirements of ANOVA. The effect of $\mathrm{CO}_{2}$ was tested against the random effect of ring nested within $\mathrm{CO}_{2}$ (Reich et al. 2001c). We did not correct for multiple statistical tests considering the mathematical and logical argumentation by Moran (2003) (Moran 2003). Briefly, ecological studies often are highly variable and have low replication resulting in more high $P$-values and only few very low $P$-values, therefore decreasing the probability of significant multiple statistical tests. Nematode guilds and families comprising less than $2 \%$ of the entire population were excluded from the analysis. Treatment effects on the overall density of varying trophic groups and taxa richness are described elsewhere (Eisenhauer et al. 2012).

\section{Results}

\section{Family composition}

Elevated $\mathrm{CO}_{2}$ significantly decreased densities of plant feeders within $c-p 5\left(\mathrm{Pl}_{5}\right)$, consisting exclusively of root-feeding Longidoridae, but the effect varied with $\mathrm{N}$ supply (Table 1, Fig. 1a). At $e \mathrm{CO}_{2}, e \mathrm{~N}$ decreased Longidoridae density significantly by $-65 \%$ compared to $a \mathrm{~N}$ at $e \mathrm{CO}_{2}$. In contrast, at $a \mathrm{CO}_{2}, e \mathrm{~N}$ significantly increased Longidoridae density by $+148 \%$ compared to $a \mathrm{~N}$ at $a \mathrm{CO}_{2}$. Other plant feeders did not react significantly to the treatments. Elevated $\mathrm{N}$ significantly increased densities of $\mathrm{Ba}_{2}$ (Table 1), mainly due to dominant bacterial-feeding 
a)

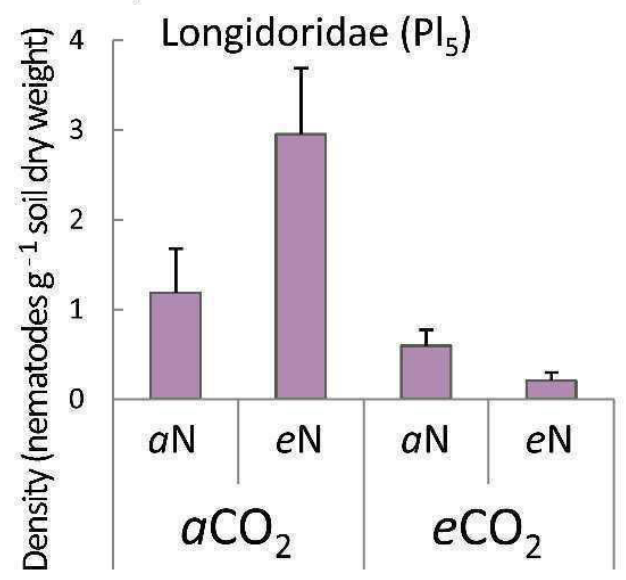

c)

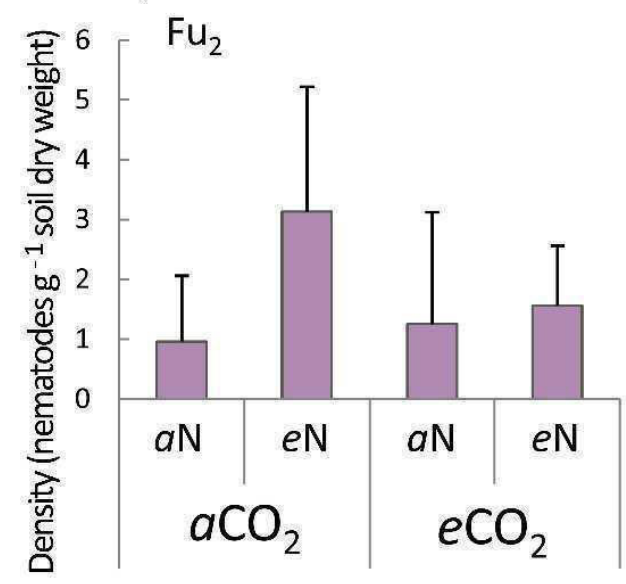

b)

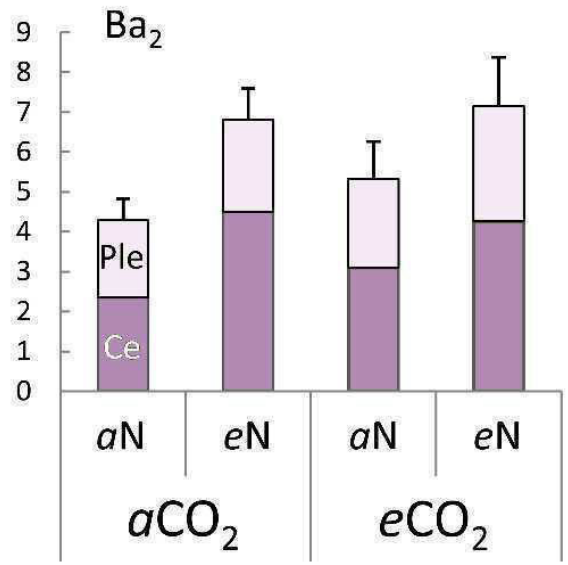

d)

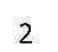

2

Mononchidae $\left(\mathrm{Pr}_{4}\right)$

1

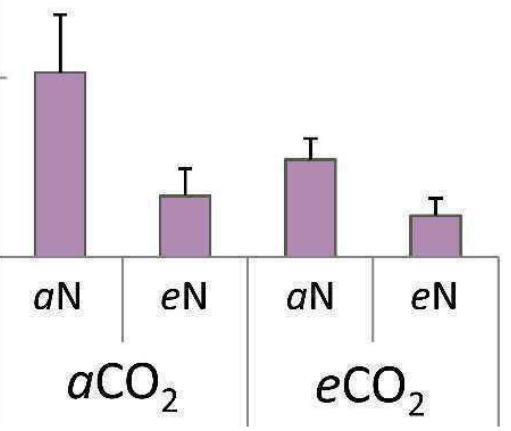

Figure 1 The effect of $\mathrm{CO}_{2}\left(a \mathrm{CO}_{2}=\right.$ ambient $\mathrm{CO}_{2}, e \mathrm{CO}_{2}=$ elevated $\left.\mathrm{CO}_{2}\right)$ and $\mathrm{N}$ addition $(a \mathrm{~N}=$ ambient $\mathrm{N}, e \mathrm{~N}=$ elevated $\mathrm{N}$ ) on a) Longidoridae $\left.\left(\mathrm{Pl}_{5}\right), \mathrm{b}\right) \mathrm{Ba} 2$ nematodes, c) Fu2 nematodes and d) Mononchidae $\left(\operatorname{Pr}_{4}\right)$. $\mathrm{PI}=\mathrm{plant}$ feeders, $\mathrm{Ba}=$ bacterial feeders, $\mathrm{Fu}=$ fungal feeders, $\mathrm{Pr}=$ predators, $\mathrm{Ce}=$ Cephalobidae, $\mathrm{Ple}=$ Plectidae. Subscript numbers refer to the related $c$ - $p$-classification of nematodes (see text for details). Means $\pm S E ; n=6$.

Cephalobidae increasing in density by $+61 \%$ at $e \mathrm{~N}$ compared to $a \mathrm{~N}$ (Fig. $1 \mathrm{~b}$; Table 2 ). No bacterial-feeding guild was significantly influenced by $\mathrm{CO}_{2}$ or precipitation nor by any interaction (Table 1).

Densities of $\mathrm{Fu}_{2}$ were significantly higher at $e \mathrm{~N}(+112 \%)$ than at $a \mathrm{~N}$, but differences mainly occurred at $a \mathrm{CO}_{2}$ (2-way interaction; Fig. 1c).

The predatory Mononchidae, representing the guild $\mathrm{Pr}_{4}$, were significantly reduced at $e \mathrm{~N}$ compared to ambient $\mathrm{N}$ levels (-63\%; Fig. 1d), whereas omnivore nematodes were not significantly influenced by any of the treatments (Table 1). 
Table 1 ANOVA table of $F$ - and $P$-values on the effects of $\mathrm{CO}_{2}$ (ambient and elevated), $\mathrm{N}$ (ambient and elevated), summer precipitation (PREC: ambient and reduced precipitation) and all possible interactions on the abundance of functional guilds of soil nematodes characterized with the same feeding habit (trophic group) and by life history characteristics expressed along a colonizer-persister ( $c-p)$ scale (after Bongers 1990) as indicated by the number on the $c-p$ scale and on nematode indices after Bongers (1990) and Ferris et al. (2001). PI = plant feeders, Ba = bacterial feeders, Fu = fungal feeders, Om $=$ omnivors, Pr $=$ predators, subscript numbers indicate the corresponding $c-p$ classification, $\mathrm{EI}=$ Enrichment Index, $\mathrm{SI}=$ Struct ure Index, $\mathrm{Cl}=$ Channel Index. Arrows indicate the direction of treatment effects. $\uparrow=$ increase in abundance at elevated $\mathrm{CO}_{2}$ and elevated $\mathrm{N}$ or at reduced level of precipitation, $\downarrow=$ decrease. Rare groups are not displayed and were not analyzed with ANOVA due to very low densities (<2\%). For means consider Table S2

\begin{tabular}{|c|c|c|c|c|c|c|c|c|c|c|c|c|c|c|c|c|}
\hline & \multicolumn{3}{|c|}{$\mathrm{CO}_{2}$} & \multicolumn{2}{|r|}{$\mathbf{N}$} & & \multicolumn{2}{|r|}{ PREC } & \multicolumn{2}{|c|}{$\mathrm{CO}_{2} \times \mathrm{N}$} & \multicolumn{2}{|c|}{$\mathrm{CO}_{2} \times \mathrm{PREC}$} & \multicolumn{2}{|c|}{$\mathbf{N} \times$ PREC } & \multicolumn{2}{|c|}{$\mathrm{CO}_{2} \times \mathrm{N} \times \mathrm{PREC}$} \\
\hline & $F$ & $P$ & & $F$ & $P$ & & $F$ & $P$ & $\boldsymbol{F}$ & $P$ & $\boldsymbol{F}$ & $P$ & $\boldsymbol{F}$ & $P$ & $F$ & $P$ \\
\hline \multicolumn{17}{|c|}{$c-p$-classes } \\
\hline$c-p 1$ & 1.47 & 0.2930 & & 1.58 & 0.2162 & & 0.10 & 0.7591 & 0.23 & 0.6316 & 3.09 & 0.0873 & 0.06 & 0.8079 & 0.05 & 0.8278 \\
\hline$c-p 2$ & 0.01 & 0.9215 & & 5.14 & 0.0294 & $\uparrow$ & 0.02 & 0.8879 & 0.78 & 0.3827 & 0.05 & 0.8298 & 0.97 & 0.3320 & 0.73 & 0.3970 \\
\hline$c-p 3$ & 0.10 & 0.7700 & & 0.01 & 0.9141 & & 1.99 & 0.1673 & 0.00 & 0.9967 & 0.81 & 0.3727 & 0.02 & 0.8832 & 2.84 & 0.1004 \\
\hline$c-p 4$ & 0.15 & 0.7206 & & 9.16 & 0.0046 & $\downarrow$ & 1.74 & 0.1949 & 3.17 & 0.0835 & 0.10 & 0.7590 & 2.28 & 0.1399 & 0.96 & 0.3346 \\
\hline$c-p 5$ & 2.56 & 0.1857 & & 1.26 & 0.2688 & & 0.15 & 0.7039 & 1.45 & 0.2371 & 0.12 & 0.7280 & 0.90 & 0.3498 & 0.01 & 0.9087 \\
\hline \multicolumn{17}{|c|}{ Functional guilds } \\
\hline $\mathrm{Pl}_{2}$ & 0.00 & 0.9936 & & 0.01 & 0.9386 & & 1.83 & 0.1851 & 0.50 & 0.4861 & 0.02 & 0.8951 & 0.06 & 0.8003 & 0.00 & 0.9603 \\
\hline $\mathrm{Pl}_{5}$ & 4.89 & 0.0922 & & 0.88 & 0.3544 & & 0.05 & 0.8203 & 7.06 & 0.0117 & 0.00 & 0.9563 & 0.25 & 0.6210 & 0.12 & 0.7295 \\
\hline $\mathrm{Ba}_{1}$ & 1.47 & 0.2930 & & 1.58 & 0.2162 & & 0.10 & 0.7591 & 0.23 & 0.6316 & 3.09 & 0.0873 & 0.06 & 0.8079 & 0.05 & 0.8278 \\
\hline $\mathrm{Ba}_{2}$ & 0.06 & 0.8118 & & 7.16 & 0.0112 & $\uparrow$ & 1.36 & 0.2506 & 0.33 & 0.5669 & 0.12 & 0.7331 & 0.51 & 0.4786 & 2.93 & 0.0956 \\
\hline $\mathrm{Ba}_{3}$ & 0.09 & 0.7804 & & 0.06 & 0.8034 & & 2.53 & 0.1204 & 0.01 & 0.9143 & 0.41 & 0.5256 & 0.03 & 0.8616 & 3.70 & 0.0623 \\
\hline $\mathrm{Fu}_{2}$ & 0.49 & 0.5230 & & 5.35 & 0.0266 & $\uparrow$ & 1.73 & 0.1964 & 4.30 & 0.0454 & 0.00 & 0.9870 & 0.81 & 0.3745 & 0.05 & 0.8280 \\
\hline $\mathrm{Fu}_{4}$ & 0.55 & 0.4995 & & 2.23 & 0.1437 & & 2.75 & 0.1059 & 3.35 & 0.0757 & 0.06 & 0.8007 & 0.06 & 0.8091 & 1.94 & 0.1721 \\
\hline $\mathrm{Om}_{4}$ & 1.24 & 0.3292 & & 2.44 & 0.1267 & & 1.60 & 0.2145 & 2.14 & 0.1517 & 0.08 & 0.7744 & 2.23 & 0.1437 & 0.14 & 0.7118 \\
\hline $\mathrm{Om}_{5}$ & 0.19 & 0.6842 & & 0.24 & 0.6291 & & 0.46 & 0.5035 & 1.48 & 0.2312 & 0.09 & 0.7696 & 0.48 & 0.4914 & 0.13 & 0.7240 \\
\hline $\mathrm{Pr}_{4}$ & 1.16 & 0.3444 & & 7.68 & 0.0088 & $\downarrow$ & 0.09 & 0.7677 & 0.16 & 0.6931 & 0.16 & 0.6894 & 1.94 & 0.1717 & 0.11 & 0.7404 \\
\hline \multicolumn{17}{|l|}{ Indices } \\
\hline $\mathrm{El}$ & 6.42 & 0.0688 & & 11.97 & 0.0014 & $\downarrow$ & 1.38 & 0.2480 & 0.17 & 0.6812 & 5.20 & 0.0286 & 0.59 & 0.4492 & 0.38 & 0.5429 \\
\hline SI & 10.67 & 0.0419 & $\downarrow$ & 10.09 & 0.0031 & $\downarrow$ & 0.00 & 0.9813 & 0.02 & 0.9030 & 0.12 & 0.7325 & 0.85 & 0.3638 & 0.92 & 0.3439 \\
\hline $\mathrm{Cl}$ & 1.84 & 0.2470 & & 10.59 & 0.0025 & $\uparrow$ & 0.56 & 0.4572 & 2.25 & 0.1423 & 4.19 & 0.0481 & 2.94 & 0.0950 & 0.83 & 0.3682 \\
\hline
\end{tabular}


Table 2 | Mean \pm SE of abundance (Individuals/g soil dry weig ht) for $c-p$ scale and functional guilds as well as Enrichment Index (EI), Structure Index (SI) and Channel Index (CI) as affected by of $\mathrm{CO} 2$ (ambient $=a \mathrm{CO} 2$, elevated $=e \mathrm{CO} 2$ ), nitrogen ( $a$ mbient $=a \mathrm{~N}$, elevated $e \mathrm{~N}$ ) and precipitation (ambient $=a \mathrm{PREC}$, elevated $=r \mathrm{PREC})$. Pl= plant feeders,

$\mathrm{Ba}=$ bacterial feeders, $\mathrm{Fu}=$ fungal feeders, $\mathrm{Om}=$ omnivors, $\mathrm{Pr}=$ predators, subscript numbers indicate the corresponding c-p classification. ${ }^{*}=$ not analyze $d$ with ANOVA due to very low densities $(<2 \%)$

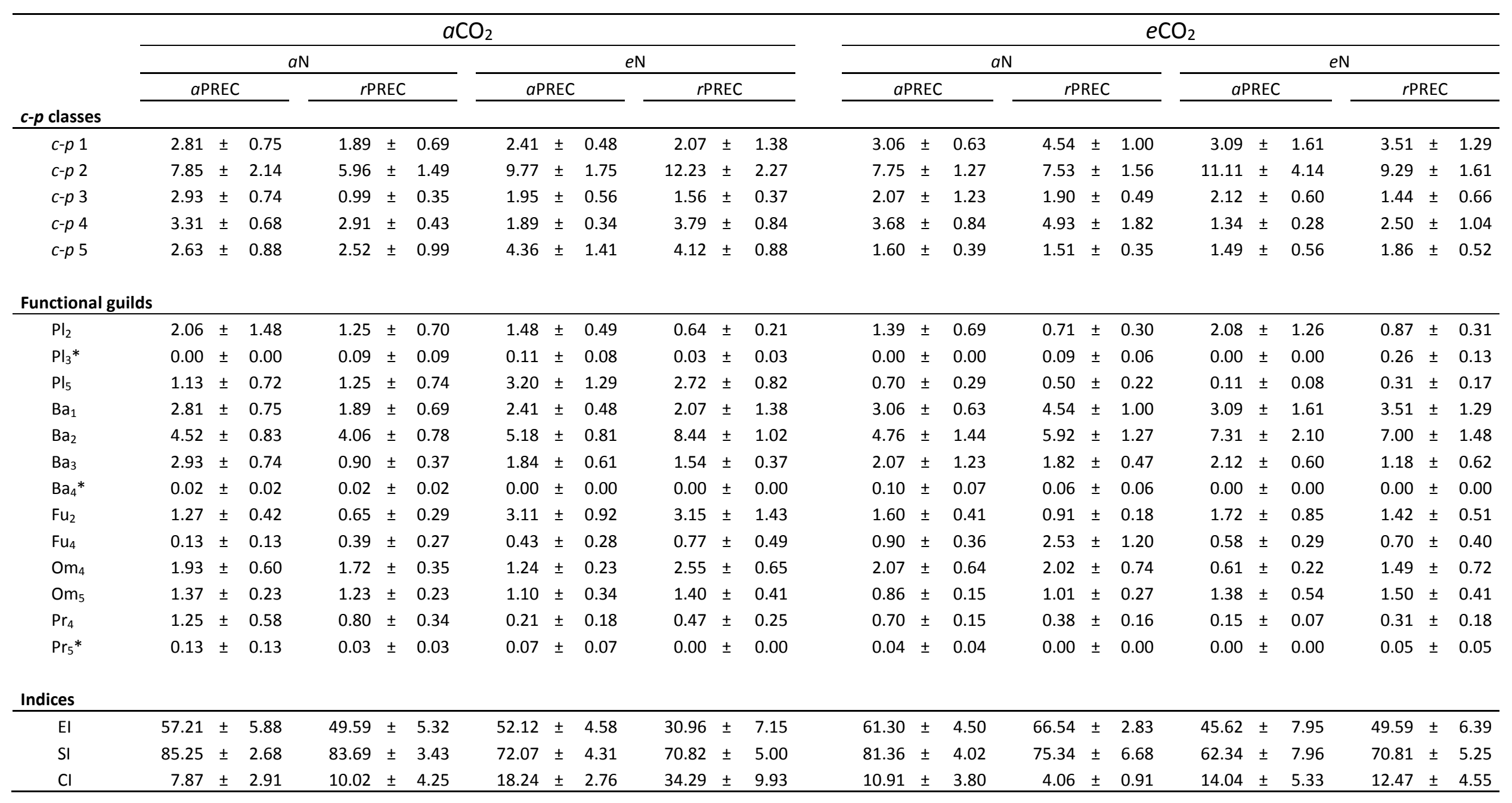




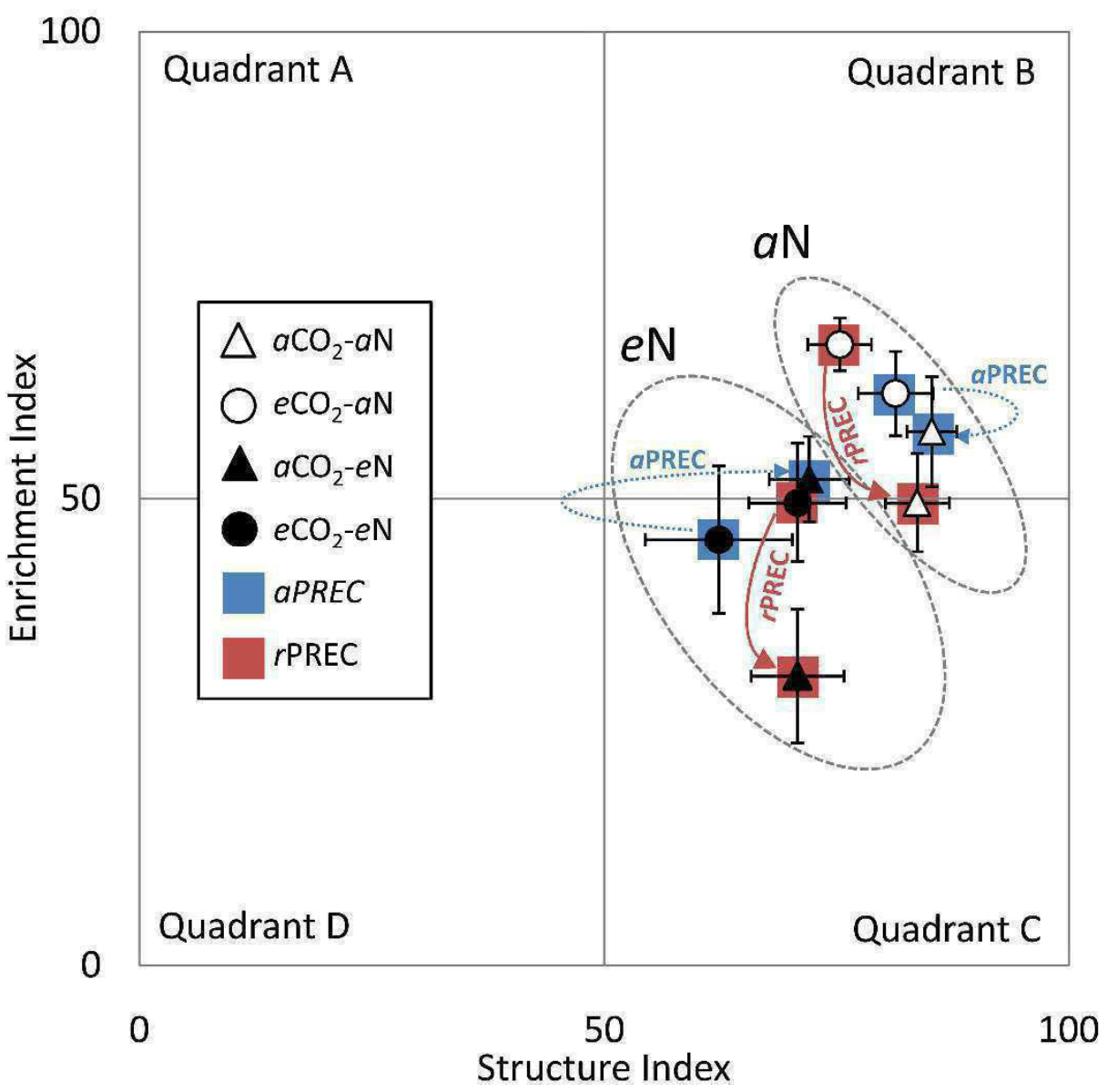

Figure 2 | Enrichment Index (EI) and Structure Index (SI) trajectories for plots arranged in a full factorial combination of ambient $\mathrm{CO}_{2}\left(a \mathrm{CO}_{2}\right.$; triangles) or elevated $\mathrm{CO}_{2}\left(e \mathrm{CO}_{2}\right.$; circles), ambient $\mathrm{N}$ ( $a \mathrm{~N}$; open symbols) or elevated $\mathrm{N}$ (eN; filled symbols) as well as ambient precipitation ( $a$ PREC; blue squares) and reduced precipitation ( $r$ PREC; red squares). Ellipses group $\mathrm{N}$ treatments. Continious arrows indicate effects of $r$ PREC reducing $\mathrm{EI}$, whereas dotted arrows show only little effects of aPREC on $\mathrm{EI},(n=6)$.

\section{Functional guilds}

Different components of the nematode community were influenced by the three environmental factors - most strongly by $\mathrm{N}$, modestly by $\mathrm{CO}_{2}$ and not at all by precipitation. Interactions were found mainly for $\mathrm{CO}_{2} \times \mathrm{N}$ on the level of nematode functional guilds, and for $\mathrm{CO}_{2} \times$ PREC in case of nematode indices. The BioCON site can be classified according to the position of samples within the faunal profile based on EI and SI (Fig. 2). The varying global change treatments were plotted closely dispersing in quadrants B and C. Generally, quadrant B classifies sites being little to moderately disturbed, $\mathrm{N}$ enriched, with a balanced 


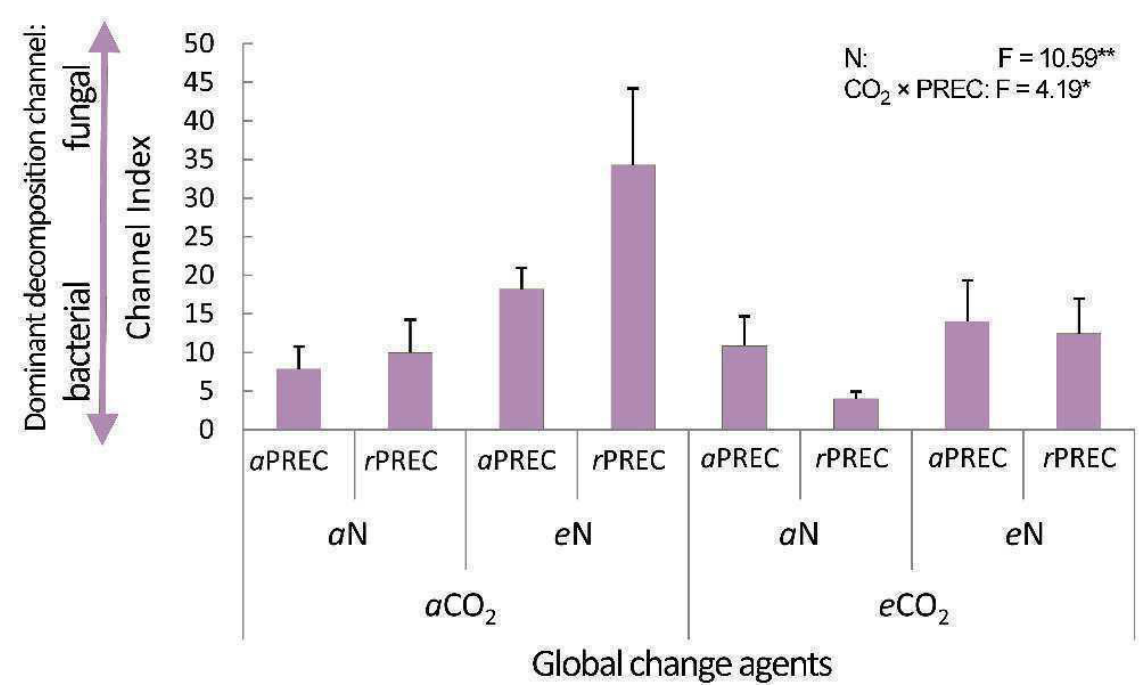

Figure 3 | The relative contribution (mean $\pm \mathrm{SE}$ ) of bacterial feeders within $c-p 1\left(\mathrm{Ba}_{1}\right)$ and fungal feeders within $c-p 2\left(\mathrm{Fu}_{2}\right)$ reflect the importance of decomposition channels as affected by $\mathrm{CO}_{2}\left(a \mathrm{CO}_{2}=\right.$ ambient $\mathrm{CO}_{2}$, $e \mathrm{CO}_{2}=$ elevated $\left.\mathrm{CO}_{2}\right), \mathrm{N}$ addition $(a \mathrm{~N}=$ ambient $\mathrm{N}, e \mathrm{~N}=$ elevated $\mathrm{N})$ and precipitation $(a \mathrm{PREC}=$ ambient precipitation, $r$ PREC $=$ reduced precipitation). Low $\mathrm{Cl}$ values indicate bacterial dominated decomposition, whereas high values refer to a more fungal dominated system $(n=6)$.

decomposition channel (i.e., bacterial vs. fungal-f eeding nematodes), and a mature food web, whereas quadrant $\mathrm{C}$ contains undisturbed sites, moderate enrichment, decomposition channels dominated by fungi and stable food web con ditions. Here, quadrant B contained plots treated with ambient $\mathrm{N}$, whereas quadrant $\mathrm{C}$ comprised plots with $\mathrm{N}$ addition, indicating a shift in decomposition channels towards fungi at $\mathrm{eN}$. In detail, nematode communities at $\mathrm{eN}$ were significantly less structured and enriched than at $a \mathrm{~N}$, as indicated by El and SI (Fig. 2, Table 1). In addition, nematode densities belonging to $c-p 2$ generally were significantly increased at $e \mathrm{~N}$ as compared to $a \mathrm{~N}(+46 \%)$, whereas nematodes of the $c-p 4$ class were significantly reduced at eN (-36\%; Table 1). Moreover, nematode communities were significantly less structured at $e \mathrm{CO}_{2}$ than at $a \mathrm{CO}_{2}$. The El was reduced by $r \mathrm{PREC}$ at $a \mathrm{CO}_{2}$, but not by aPREC.

The $\mathrm{Cl}$ indicated that decomposition pathways at the BioCON site ranged from strongly bacterial dominated to balanced energy channels (Fig. 3). Reduced precipitation significantly increased $\mathrm{Cl}$ at $a \mathrm{CO}_{2}$, whereas $\mathrm{Cl}$ was lowest at $e \mathrm{CO}_{2}$ and $r \mathrm{PREC}$. $\mathrm{Cl}$ was significantly higher at 
$e \mathrm{~N}$ than at $a \mathrm{~N}(-58 \%)$, indicating stronger fungal contribution to decomposition processes at $e \mathrm{~N}$, whereas decomposition was mainly driven by bacteria at $a \mathrm{~N}$ (Table 1 ).

\section{Discussion}

We used nematodes as indicators of soil ecological processes as affected by elevated $\mathrm{CO}_{2}$, enriched $\mathrm{N}$ and reduced precipitation as well as the ir interactions in a long-term global change experiment in grassland. Functional guilds of nematodes were significantly influenced by some of the treatments. In particular, elevated $\mathrm{N}$ and interactions between $\mathrm{N}$ and $\mathrm{CO}_{2}$ altered the structure of nematode communities, suggesting complex changes in belowground processes in a changing world.

Hypothesis (1) posited that $e \mathrm{CO}_{2}$ increases the abundance of opportunistic nematodes, which was not supported by our results. Indeed, however, the availability of carbon sources within the soil system increased as indicated by significantly higher labile carbon flow (Adair et al. 2011) and higher soil microbial biomass (Eisenhauer et al. 2012) at the same field site, but all nematode groups remained unaffected. Similar to our results, Hungate et al. (2000) and Yeates et al. (1997) reported no increase in bacterial-feeding nematodes despite higher microbial biomass. They argued that top-down forces, e.g. caused by increased densities of predatory microarthropods and nematodes, counteracted bottom-up effects. Indeed, eCO increased the density of gamasid mites $(+143 \%)$ and Collembola $(+64 \%)$ in the BioCON experiment (Eisenhauer et al. 2012), and both groups are known to feed on nematodes (Ruess et al. 2005; Klarner et al. 2012). In addition, the abundance of ciliates increased at $e \mathrm{CO}_{2}$ by $+83 \%$ (Eisenhauer et al. 2012), and this may have also circumvented bottom-up effects on bacterial-feeding nematodes due to resource competition (Bergtold et al. 2005). Thus, 
elevated availability of labile $\mathrm{C}$ compounds in this soil at $e \mathrm{CO}_{2}$ might have affected competitively superior groups of soil biota, and therefore is likely to induce shifts in the composition of soil food webs by altering the prevalence of bottom-up vs. top-down forces.

Hypothesis (2), assuming $e \mathrm{~N}$ to reduce food web structure of the nematode community but to increase opportunistic nematodes, was supported by our data. Elevated $\mathrm{N}$ availability significantly increased the density of opportunistic $\mathrm{Ba}_{2}$ nematodes, although microbial biomass did not increase at eN (Eisenhauer et al. 2012). In contrast and as detailed above, microbial biomass increased at $e \mathrm{CO}_{2}$ without significantly affecting any bacterial-feeding nematodes. This suggests that food quality ,i.e., n utrient concentrations of microorganisms may be of greater importance for opportunistic bacterial-feeding nematodes than food quantity as reflected by microbial biomass (Schmidt et al. 2000). An increase in the nutrient quality of microorganisms is probably induced by shifts in the quality of organic material and plant exudates delivered by plants (Bardgett and Wardle 2010). The lack of response in $\mathrm{Ba}_{1}$ nematodes is probably due to an increase in densities rapidly after resource increases (Bongers and Ferris 1999), thus sampling five weeks after the last experimental $\mathrm{N}$ addition may not reflect a prolonged increase in this group. This is in line with other studies were members of $\mathrm{Ba}_{1}$ did respond with little or no changes to $\mathrm{N}$ addition (Sarathchandra et al. 2001; Wei et al. 2012). The remaining bacterial feeders of higher $c-p$ classes ( $\mathrm{Ba}_{3}$ and $\left.\mathrm{Ba}_{4}\right)$ decreased in numbers which supports hypothesis (2) as mainly opportunistic nematodes profit from $\mathrm{N}$ addition and as food web structure was reduced. This is also supported by the strong reduction of predatory nematodes at $e \mathrm{~N}$. Since omnivores and predatory nematodes predominate in stable and structured environments (Bongers 1990; Ferris et al. 2001), their reduced densities likely indicate disturbed conditions. The dominant predatory taxon at the field site of the BioCON experiment Mononchidae mainly feeds on other nematodes (Loof 
1999). However, Mononchidae did not benefit from increased nematode densities at $\mathrm{eN}$ likely causing short nutrient flushes only favoring fast g rowing opportunists. Thus, our results point to a less structured food web with a lower number of trophic levels and reduced top-down forces at $e \mathrm{~N}$. This is also indicated by decreased plant (Wedin and Tilman 1996) and soil fauna diversity (Eisenhauer et al. 2012) at $e \mathrm{~N}$, resulting in a loss of functional traits (Suding et al. 2005).

Generally, effects of $\mathrm{N}$ fertilization on soil microbial activity are little understood (Dijkstra et al. 2005), but several studies stressed that decomposition is predominantly negatively to neutrally influenced by $\mathrm{N}$ fertilization (Hobbie 2000). At our study site, $\mathrm{N}$ fertilization only slightly increased decomposition (Knops et al. 2007). However, the decomposition channel changed as indicated by higher $\mathrm{Cl}$ at $e \mathrm{~N}$, reflecting a stronger fungal contribution to decomposition processes at $e \mathrm{~N}$, potentially due to decreasing C-to-N ratios of organic material (Ruess 2003; Ruess and Ferris 2004) and reduced carbon allocation to roots (Högberg et al. 2010). Negative effects of $e \mathrm{~N}$ on the labile $\mathrm{C}$ pool are supported by a recent structural equation modeling approach by Eisenhauer et al. (2012), suggesting that negative effects of eN on several groups of soil biota were due to decreased rhizodeposition.

Hypothesis (3), assuming that reduced precipitation little affects opportunistic soil nematodes, was supported by the results of this study. Although summer precipitation was reduced by approximately $45 \%$ (Eisenhauer et al. 2012), soil nematodes were little affected. This is in line with other studies reporting only w eak effects of soil moisture on opportunistic nematodes (Porazinska et al. 1998; Ekschmitt et al. 1999). However, in combination with e $\mathrm{CO}_{2}$, $\mathrm{El}$ and $\mathrm{Cl}$ revealed compensatory effects of $e \mathrm{CO}_{2}$ to drought, as drought had little effects at $e \mathrm{CO}_{2}$. Interestingly, only $e \mathrm{CO}_{2}$ but not aPREC (as compared to reduced precipitation) led to higher soil water content (Eisenhauer et al. 2012). Potentially, moderate drought stress was 
compensated by $\mathrm{eCO}_{2}$ due to reduced transpiration as a consequence of decreased stomatal conductance (Lee et al. 2011). Overall, no responses of all nematode groups to rPREC may be explained by their ability to stand dry conditions by anhydrobiosis (Treonis et al. 2000) and as the community may generally be adapted to dry conditions as it is typical for this sandy soil.

As stressed above, nematode community indices were influenced by several interactions between global change agents. These interactions were non-additive and therefore not predictable from single factor effects supporting hypothesis (4). Fungal-feeding nematodes with $r$-strategy $\left(\mathrm{Fu}_{2}\right)$ increased on plots with $\mathrm{N}$ addition at $a \mathrm{CO}_{2}$, whereas little differences were found at $e \mathrm{CO}_{2}$. Elevated $\mathrm{CO}_{2}$ is assumed to favor arbuscular mycorrhizal fungi (AMF) due to increased root carbon supply (Jifon et al. 2002; Treseder 2004), whereas N fertilization reduced $A M$ biomass (Antoninka et al. 2011). Our results suggest that $\mathrm{Fu}_{2}$ did not rely on $\mathrm{AMF}$, as their densities increased as AMF fungi decreased (Antoninka et al. 2011), but may feed predominantly on saprotrophic fungi. In contrast, $\mathrm{Fu}_{4}$ followed the reverse pattern and reacted negatively to $\mathrm{N}$ fertilization (-63\%) and increased strongly at $e \mathrm{CO}_{2}$ and $a \mathrm{~N}$. This suggests that these functional guilds depend on different fungi, $\mathrm{Fu}_{2}$ on saprotrophic and $\mathrm{Fu}_{4}$ on AM fungi. Our results imply that altered $\mathrm{C}$ and $\mathrm{N}$ supply to differentially affect fungal communities and thereby soil processes as saprotrophic fungi are suggested to mobilize carbon while mycorrhiza to mobilize N (Hobbie and Horton 2007). Changes in the importance of functional groups of fungi due to global change agents therefore may affect carbon $\mathrm{C}$ pools in soil. However, our results only provide first hints and future studies are needed to check our hypotheses.

Densities of root-feeding Longidoridae ( $c-p 5)$ decreased considerably at $e \mathrm{CO}_{2}$ and $e \mathrm{~N}$ levels. Generally, $e \mathrm{CO}_{2}$ enhances photosynthesis and plant growth (Ainsworth and Long 2005; Lee et al. 2011). In the BioCON experiment, $e \mathrm{CO}_{2}$ significantly increased fine root biomass $(+22 \%$ in 
2010; P.B. Reich, unpublished data). However, Longidoridae did not benefit from enhanced fine root biomass, especially under $\mathrm{N}$-rich conditions. In studies of grazed $\mathrm{C}_{3}$ grass-dominated pasture on sand Yeates et al. (2003) and Yeates and Newton (2009) found densities of Longidorus elongatus (de Man) to be significantly higher at $e \mathrm{CO}_{2}$ than at $a \mathrm{CO}_{2}$ (Yeates et al. 2003; Yeates and Newton 2009). This contrasts with our findings suggesting different forces in structuring the soil food web in different envir onmental contexts, e.g., soil type, vegetation and climate. Variable responses of plant-feeding ne matodes to $\mathrm{eCO}_{2}$ were reported before (Runion et al. 1994; Yeates et al. 1997, 2003; Hoeksema et al. 2000; Hungate et al. 2000; Neher et al. 2004; Kardol et al. 2010; Wei et al. 2012), indicating that effects not solely depend on the quantity of resource inputs (when assuming a general increase in belowground inputs at $\left.e \mathrm{CO}_{2}\right)$ (Adair et al. 2011). Moreover, the interactions between $\mathrm{CO}_{2}$ and $\mathrm{N}$ argue for multifactor experiments but also to consider different biotic (plant and animal community, SOM) and abiotic contexts (soil type, latitude) to correctly interpret different patterns. In addition, it was shown experimentally for $\mathrm{C}_{3}$ plants that high $\mathrm{CO}_{2}$ concentrations increased $\mathrm{C}$ - but also $\mathrm{N}$ containing compounds like alkaloids (Ziska et al. 2005; Matros et al. 2006; Bidart-Bouzat and Imeh-Nathaniel 2008), suggesting enhanced investment in plant defense (Stiling and Cornelissen 2007; Sun et al. 2011). Thus, changes in the composition of secondary plant compounds due to elevated $\mathrm{C}$ and $\mathrm{N}$ availability may have reduced the performance of some plant feeding groups in our experiment. In contrast, the positive effect of $e \mathrm{~N}$ at $a \mathrm{CO}_{2}$ on Longidoridae reflect a common fertilization effect due to increased plant biomass $\mathrm{N}$ content in plant tissue (Reich et al. 2006b), with the latter likely representing high quality food. However, other plant-feeding nematodes, such as Tylenchidae, were not significantly affected suggesting that those taxa are more tolerant to changes in plant physiology. In sum, changes in $\mathrm{C}$ and $\mathrm{N}$ regimes may alter plant performance and consequently impact on trophic 
interactions; however, varying outcomes due to differences in species traits make generalizations difficult (Reich et al. 2006a).

Our results show the relevance of interactive effects of $\mathrm{CO}_{2}, \mathrm{~N}$ and precipitation on nematode communities and their functions, suggesting that long-term studies on interactive effects of global change agents are needed to improve predictions of consequences of future global changes (Reich et al. 2012). Increased carbon allocation to roots and mycorrhizal fungi and high $\mathrm{N}$ inputs presumably changed the composition of the microbial community with cascading effects on the soil food web and on soil processes. The shift in soil energy channels towards $r$-strategists at elevated $\mathrm{N}$ levels points to significant changes in the functioning of soil food webs. Future studies need to investigate consequences for soil $\mathrm{C}$ dynamics to better predict if soils act as $\mathrm{C}$ source or $\mathrm{C}$ sink when subjected to climate change. Moreover, studies in global change experiments are needed relating changes in the functional indices of soil nematodes to process rates, such as C dynamics with considering aboveground and belowground systems together as they are intimately linked.

\section{Acknowledgements}

We thank A. Jousset for helpful comments on the first manuscript version, Molly Heit for help during field sampling, and Cindy Buschena, Montara Roberts, Chris Bergquist and Susan Barrott (University of Minnesota) for their help during the processing of soil samples. Simone Cesarz was funded by the Ministry of Science and Culture of Lower Saxony and the 'Niedersächsisches Vorab' as part of the Cluster of Excellence 'Functional Biodiversity Research'. Nico Eisenhauer gratefully acknowledges funding by the Deutsche Forschungsgemeinschaft (DFG; Ei 862/1). This work was supported by the Department of 
Energy (DOE/DE-FG02-96ER62291) and the National Science Foundation (NSF Biocomplexity

0322057, NSF LTER DEB 9411972 (1994-2000), DEB 0080382 (2000-2006), and DEB 0620652

(2006-2012), and NSF LTREB 0716587). Further, this research was supported by the Department of Energy, Office of Science (BER) through the Midwestern Regional Center of the National Institute for Climatic Change Research at Michigan Technological University (DEFC02-06ER64158).

\section{References}

Adair, E.C., Reich, P.B., Trost, J.J., Hobbie, S.E., 2011. Elevated $\mathrm{CO}_{2}$ stimulates grassland soil respiration by increasing carbon inputs rather than by enhancing soil moisture. Global Change Biology 17, 3546-3563.

Ainsworth, E.A., Long, S.P., 2005. What have we learned from 15 years of free-air $\mathrm{CO}_{2}$ enrichment (FACE)? A meta-analytic review of the responses of photosynthesis, canopy properties and plant production to rising $\mathrm{CO}_{2}$. New Phytologist 165, 351-371.

Antoninka, A., Reich, P.B., Johnson, N.C., 2011. Seven years of carbon dioxide enrichment, nitrogen fertilization and plant diversity influence arbuscular mycorrhizal fungi in a grassland ecosystem. New Phytologist 192, 200-214.

Bardgett, R.D., Wardle, D.A., 2010. Aboveground-Belowground Linkages, Biotic Interactions, Ecosystem Processes, and Global Change. Oxford Series in Ecology and Evolution, Oxford University Press, New York, USA.

Bergtold, M., Gunter, V., Traunspurger, W., 2005. Is there competition among ciliates and nematodes? Freshwater Biology 50, 1351-1359.

Bidart-Bouzat, M.G., Imeh-Nathaniel, A., 2008. Global change effects on plant chemical defenses against insect herbivores. Journal of Integrative Plant Biologytegrative Plant Biology 50, 1339-1354.

Blankinship, J.C., Niklaus, P.A., Hungate, B.A., 2011. A meta-analysis of responses of soil biota to global change. Oecologia 165, 553-65.

Bongers, T., 1990. The maturity index: an ecological measure of environmental disturbance based on nematode species composition. Oecologia 83, 14-19. 
Bongers, T., Ferris, H., 1999. Nematode community structure as a bioindicator in environmental monitoring. Trends in Ecology \& Evolution 14, 224-228.

Chung, H., Zak, D.R., Reich, P.B., Ellsworth, D.S., 2007. Plant species richness, elevated $\mathrm{CO}_{2}$, and atmospheric nitrogen deposition alter soil microbial community composition and function. Global Change Biology 13, 980-989.

Clark, C.M., Tilman, D., 2008. Loss of plant species after chronic low-level nitrogen deposition to prairie grasslands. Nature $451,712-5$.

Dijkstra, F.A., Hobbie, S.E., Reich, P.B., Knops, J.M.H., 2005. Divergent effects of elevated $\mathrm{CO}_{2}, \mathrm{~N}$ fertilization, and plant diversity on soil $\mathrm{C}$ and $\mathrm{N}$ dynamics in a grassland field experiment. Plant and Soil 272, 41-52.

Drigo, B., Pijl, A.S., Duyts, H., Kielak, A.M., Gamper, H.A., Houtekamer, M.J., Boschker, H.T.S., Bodelier, P.L.E., Whiteley, A.S., Van Veen, J.A., Kowalchuk, G.A., 2010. Shifting carbon flow from roots into associated microbial communiti es in response to elevated atmospheric $\mathrm{CO}_{2}$. Proceedings of the National Academy of Sciences of the United States of America 107, 10938-10942.

Eisenhauer, N., Cesarz, S., Koller, R., Worm, K., Reich, P.B., 2012. Global change belowground: impacts of elevated $\mathrm{CO}_{2}$, nitrogen, and summer drought on soil food webs and biodiversity. Global Change Biology 18, 43 5-447.

Ekschmitt, K., Bakonyi, G., Bongers, M., Bongers, T., Boström, S., Anthony, G., Dogan, H., Harrison, A., Kallimanis, A., Donnell, O., Sohlenius, B., Stamou, G.P., Wolters, V., 1999. Effects of the nematofauna on microbial energy and matter transformation rates in European grassland soils. Plant and Soil 212, 45-61.

Ferris, H., 2010. Contribution of nematodes to the structure and function of the soil food web. Journal of Nematology 42, 63-67.

Ferris, H., Bongers, T., De Goede, R.G.M., 2001. A framework for soil food web diagnostics: extension of the nematode faunal analysis concept. Applied Soil Ecology 18, 13-29.

Ferris, H., Eyre, M., Venette, R.C., Lau, S.S., 1996. Population energetics of bacterial-feeding nematodes: Stage-specific development and fecundity rates. Soil Biology and Biochemistry 28, 271-280.

Gessner, M.O., Swan, C.M., Dang, C.K., McKie, B.G., Bardgett, R.D., Wall, D.H., Hättenschwiler, S., 2010. Diversity meets decomposition. Trends in Ecology \& Evolution 25, 372-380.

Grayston, S.J., Campbell, C.D., Lutze, J.L., Gifford, R.M., 1998. Impact of elevated $\mathrm{CO}_{2}$ on the metabolic diversity of microbial communities in $\mathrm{N}$-limited grass swards. Plant and Soil 203, 289-300. 
Gross, N., Robson, T.M., Lavorel, S., Albert, C., Le Bagousse-Pinguet, Y., Guillemin, R., 2008. Plant response traits mediate the effects of subalpine grasslands on soil moisture. New Phytologist 180, 652-662.

Hawkes, C. V., Kivlin, S.N., Rocca, J.D., Huguet, V., Thomsen, M. a., Suttle, K.B., 2011. Fungal community responses to precipitation. Global Change Biology 17, 1637-1645.

He, Z., Xu, M., Deng, Y., Kang, S., Kellogg, L., Wu, L., Van Nostrand, J.D., Hobbie, S.E., Reich, P.B., Zhou, J., 2010. Metagenomic analysis reveals a marked divergence in the structure of belowground microbial communities at elevated $\mathrm{CO}_{2}$. Ecology Letters 13, 564-575.

Hobbie, E.A., Horton, T.R., 2007. Evidence that saprotrophic fungi mobilise carbon and mycorrhizal fungi mobilise nitrogen during litter decomposition. New Phytologist 173, 447-449.

Hobbie, S.E., 2000. Interactions between litter lignin and soil nitrogen availability during leaf litter decomposition in a hawaiian montane forest. ecosystems 3, 484-494.

Hoeksema, J.D., Lussenhop, J., Teeri, J.A., 2000. Soil nematodes indicate food web responses to elevated atmospheric $\mathrm{CO}_{2}$. Pedobiologia 735, 725-735.

Högberg, M.N., Briones, M.J.I., Keel, S.G., Metcalfe, D.B., Campbell, C., Midwood, A.J., Thornton, B., Hurry, V., Linder, S., Näsholm, T., Högberg, P., 2010. Quantification of effects of season and nitrogen supply on tree below-ground carbon transfer to ectomycorrhizal fungi and other soil organisms in a boreal pine forest. New Phytologist 187, 485-493.

Hu, S., Chapin, F.S., Firestone, M.K., Field, C.B., Chiariello, N.R., 2001. Nitrogen limitation of microbial decomposition in a grassland under elevated $\mathrm{CO}_{2}$. Nature 409, 188-191.

Hungate, B.A., Jaeger III, C.H., Gamara, G., Chapin III, F.S., Field, C.B., 2000. Soil microbiota in two annual grasslands: responses to elevated atmospheric $\mathrm{CO}_{2}$. Oecologia 124, 589598.

IPCC, 2007. Climate Change 2007: The Physical Science Basis. Contribution of Working Group I to the Fourth Assessment Report of the Intergovernmental Panel on Climate Change. Cambridge University Press, Cambridge, United Kingdom and New York, NY, USA.

Jifon, J.L., Graham, J.H., Drouillard, D.L., Syvertsen, J.P., 2002. Growth depression of mycorrhizal Citrus seedlings grown at high phosphor us supply is mitigated by elevated $\mathrm{CO}_{2}$. New Phytologist 153, 133-142.

Kardol, P., Cregger, M.A., Campany, C.E., Classen, A.T., 2010. Soil ecosystem functioning under climate change: plant species and community effects. Ecology 91, 767-781.

Keeler, B.L., Hobbie, S.E., Kellogg, L.E., 2008. Effects of long-term nitrogen addition on microbial enzyme activity in eight forested and grassland sites: implications for litter and soil organic matter decomposition. Ecosystems 12, 1-15. 
Kerr, R.A., 2007. Global warming is changing the world. Science 316, 188-190.

Klarner, B., Maraun, M., Scheu, S., 2012. Trophic diversity and niche partitioning in a species rich predator guild - Natural variations in stable isotope ratios (13C/12C, 15N/14N) of mesostigmatid mites (Acari, Mesostigmata) from Central European beech forests. Soil Biology and Biochemistry.

Knops, J.M.H., Naeem, S., Reich, P.B., 2007. The impact of elevated $\mathrm{CO}_{2}$, increased nitrogen availability and biodiversity on plant tissue quali ty and decomposition. Global Change Biology 13, 1960-1971.

Knorr, M., Frey, S.D., Curtis, P.S., 2005. Nitrogen additions and litter decomposition: a metaanalysis. Ecology 86, 3252-3257.

Körner, C., 2000. Biosphere responses to $\mathrm{CO}_{2}$ enrichment. Ecological Applications 10, 15901619.

Kuzyakov, Y., Gavrichkova, O., 2010. REVIEW: Time lag between photosynthesis and carbon dioxide efflux from soil: a review of mechanisms and controls. Global Change Biology $16,3386-3406$.

Lau, J.A., Tiffin, P., 2009. Elevated carbon dioxide concentrations indirectly affect plant fitness by altering plant tolerance to herbivory. Oecologia 161, 401-410.

LeBauer, D.S., Treseder, K.K., 2008. Nitrogen limitation of net primary productivity in terrestrial ecosystems is globally distributed. Ecology Letters 89, 371-379.

Lee, T.D., Barrott, S.H., Reich, P.B., 2011. Photosynthetic responses of 13 grassland species across 11 years of free-air $\mathrm{CO}_{2}$ enrichment is modest, consistent and independent of $\mathrm{N}$ supply. Global Change Biology 17, 2893-2904.

Lindberg, N., Engtsson, J.B., Persson, T., 2002. Effects of experimental irrigation and drought on the composition and diversity of soil fauna in a coniferous stand. Journal of Applied Ecology 39, 924-936.

Loof, P.A.A., 1999. Nematoda, Adenophorea (Dorylaimida). Spektrum Akademischer Verlag, Heidelberg.

Matros, A., Amme, S., Kettig, B., Buck-Sorlin, G.H., Sonnewald, U., Mock, H.-P., 2006. Growth at elevated $\mathrm{CO}_{2}$ concentrations leads to modified profiles of secon dary metabolites in tobacco cv. SamsunNN and to increased resistance against infection with potato virus $Y$. Plant, Cell and Environment 29, 126-137.

Moran, M.D., 2003. Arguments for rejecting the sequential Bonferroni in ecological. OIKOS 100, 403-405.

Neher, D.A., Darby, B.J., 2009. Community indices, in: Wilson, M.J., Kakouli-Duarte, T. (Eds.), Nematodes as Environmental Indicators. CABI, Wallingford, UK, pp. 107-123. 
Neher, D.A., Weicht, T.R., Moorhead, D.L., Sinsabaugh, R.L., 2004. Elevated $\mathrm{CO}_{2}$ alters functional attributes of nematode communities in fo rest soils. Functional Ecology 18, 584-591.

Parton, W., Silver, W.L., Burke, I.C., Grassens, L., Harmon, M.E., Currie, W.S., King, J.Y., Adair, E.C., Brandt, L.A., Hart, S.C., Fasth, B., 2007. Global-scale similarities in nitrogen release patterns during long-term decomposition. Science 315, 361-364.

Porazinska, L., Mcsorley, R., Duncan, L.W., Gallaher, R.N., Wheaton, T.A., Parsons, L.R., 1998. Relationships between soil chemical status, soil ne matode community, and sustainability indices. Nematropica 249-262.

Reich, P.B., 2009. Elevated $\mathrm{CO}_{2}$ reduces losses of plant diversity caused by nitrogen deposition. Science 326, 1399-1402.

Reich, P.B., Hobbie, S.E., Lee, T., Ellsworth, D.S., West, J.B., Tilman, D., Knops, J.M.H., Naeem, S., Trost, J., 2006. Nitrogen limitation constrains sustainability of ecosystem response to $\mathrm{CO}_{2}$. Nature $440,922-925$.

Reich, P.B., Hungate, B.A., Luo, Y., 2006. Carbon-nitrogen interactions in terrestrial ecosystems in response to rising atmospheric carbon dioxide. Annual Review of Ecology, Evolution, and Systematics 37, 611-636.

Reich, P.B., Knops, J., Tilman, D., Craine, J., Ellsworth, D., Tjoelker, M., Lee, T., Wedin, D., Naeem, S., Bahauddin, D., Hendrey, G., Jose, S., Wrage, K., Goth, J., Bengston, W., 2001. Plant diversity enhances ecosystem responses to elevated $\mathrm{CO}_{2}$ and nitrogen deposition. Nature 411, 809-812.

Reich, P.B., Peterson, D.W., Wedin, D.A., Wrage, K., 2001. Fire and vegetation effects on productivity and nitrogen cycling across a forest. Ecology 82, 1703-1719.

Reich, P.B., Tilman, D., Craine, J., Ellsworth, D., Tjoelker, M.G., Knops, J., Wedin, D., Naeem, S., Bahauddin, D., Goth, J., Bengtson, W., Lee, T.D., 2001. Do species and functional groups differ in acquisition and use of $\mathrm{C}, \mathrm{N}$ and wa ter under varying atmospheric $\mathrm{CO}_{2}$ and $\mathrm{N}$ availability regimes? A field test with 16 grassland species. New Phytologist 150, 435-448.

Reich, P.B., Tilman, D., Isbell, F., Mueller, K., Hobbie, S.E., Flynn, D.F.B., Eisenhauer, N., 2012. Impacts of biodiversity loss escalate through time as redundancy fades. Science 336, 589-592.

Rogelj, J., Meinshausen, M., Knutti, R., 2012. Global warming under old and new scenarios using IPCC climate sensitivity range estimates. Nature Climate Change 2, 248-253.

Ruess, L., 1995. Studies on the nematode fauna of an acid forest soil: spatial disturbance and extraction. Nematologica 41, 229-239.

Ruess, L., 2003. Nematode soil faunal analysis of d ecomposition pathways in different ecosystems. Nematology 5, 179-181. 
Ruess, L., Ferris, H., 2004. Decomposition pathways and successional changes. Nematology Monographs \& Perspectives 2, 547-556.

Ruess, L., Tiunov, A., Haubert, D., Richnow, H.H., Häggblom, M.M., Scheu, S., 2005. Carbon stable isotope fractionation and trophic transfer of fatty acids in fungal based soil food chains. Soil Biology and Biochemistry 37, 945-953.

Runion, G.B., Curl, E.A., Rogers, H.H., Backman, P.A., 1994. Effects of free-air $\mathrm{CO}_{2}$ enrichment on microbial populations in the rhizosphere and phy llosphere of cotton. Agricultural and Forest Meteorology 70, 117-130.

Sarathchandra, S.., Ghani, a, Yeates, G.., Burch, G., Cox, N.., 2001. Effect of nitrogen and phosphate fertilisers on microbial and nematode diversity in pasture soils. Soil Biology and Biochemistry 33, 953-964.

Schlesinger, W.H., Andrews, J.A., 2000. Soil respiration and the global carbon cycle. Biogeochemistry 48, 7-20.

Schmidt, I.K., Ruess, L., Bååth, E., Ekelund, F., Jonasson, S., 2000. Long-term manipulation of the microbes and microfauna of two subarctic heaths by addition of fungicide, bactericide, carbon and fertilizer. Soil Biology an d Biochemistry 32, 707-720.

Schulze, E.D., Freibauer, A., 2005. Carbon unlocked from soils. Nature 437, 11-12.

Stiling, P., Cornelissen, T., 2007. How does elevated carbon dioxide $\left(\mathrm{CO}_{2}\right)$ affect plantherbivore interactions? A field experiment and meta-analysis of $\mathrm{CO}_{2}$-mediated changes on plant chemistry and herbivore performance. Global Change Biology 13, 1823-1842.

Suding, K.N., Collins, S.L., Gough, L., Clark, C., Cleland, E.E., Gross, K.L., Milchunas, D.G., Pennings, S., 2005. Functional- and abundance-based mechanisms explain diversity loss due to $\mathrm{N}$ fertilization. Proceedings of the National Academy of Sciences of the United States of America 102, 4387-92.

Sun, Y., Yin, J., Cao, H., Li, C., Kang, L., Ge, F., 2011. Elevated $\mathrm{CO}_{2}$ influences nematodeinduced defense responses of tomato genotypes differing in the JA pathway. PloS one 6, e19751.

Swift, M.J., Heal, O.W., Anderson, J.M., 1979. Decomposition in terrestrial ecosystems. University of California Press, Berkeley.

Treonis, A.M., Wall, D.H., Virginia, R.A., 2000. The use of anhydrobiosis by soil nematodes in the Antarctic Dry Valleys. Functional Ecology 14, 460-467.

Treseder, K.K., 2004. A meta-analysis of mycorrhizal responses to nitrogen, phosphorus, and atmospheric $\mathrm{CO}_{2}$ in field studies. New Phytologist 164, 347-355.

Wardle, D.A., Verhoef, H.A., Clarholm, M., 1998. Trophic relationships in the soil microfoodweb: predicting the responses to a changing global. Global Change Biology 4, 713-727. 
Wedin, D., Tilman, D., 1996. Influence of nitrogen loading and species composition on the carbon balance of grasslands. Science 274, 1720-1723.

Wei, C., Zheng, H., Li, Q., Lü, X., Yu, Q., Zhang, H., Chen, Q., He, N., Kardol, P., Liang, W., Han, X., 2012. Nitrogen Addition Regulates Soil Nematode Community Composition through Ammonium Suppression. PloS ONE 7, e43384.

Wolf, J., Johnson, N.C., Rowland, D.L., Reich, P.B., 2003. Elevated carbon dioxide and plant diversity impact arbuscular mycorrhizal fungal spore communities. New Phytologist 1720-1723.

Wuebbles, D.J., Hayhoe, K., 2004. Climate change projections for the United States Midwest. Mitigation and Adaptation Strategies for Global Cha nge 9, 335-363.

Yeates, G.W., 2010. Nematodes in Ecological Webs, in: Encyclopedia of Life Science. John Wiley \& Sons, Ltd: Chichester, pp. 1-11.

Yeates, G.W., Bongers, T., De Goede, R.G.M., Freckman, D.W., Georgieva, S.S., 1993. Feeding habits in soil nematode families and genera - an ou tline for soil ecologist. Journal of Nematology 25, 315-331.

Yeates, G.W., Newton, P.C.D., 2009. Long-term changes in topsoil nematode populations in grazed pasture under elevated atmospheric carbon dioxide. Biology and Fertility of Soils 45, 799-808.

Yeates, G.W., Newton, P.C.D., Ross, D.J., 2003. Significant changes in soil microfauna in grazed pasture under elevated carbon dioxide. Biology and Fertility of Soils 38, 319326.

Yeates, G.W., Tate, K.R., Newton, P.C.D., 1997. Response of the fauna of a grassland soil to doubling of atmospheric carbon dioxide concentration. Biology and Fertility of Soils 25, 307-315.

Zak, D.R., Pregitzer, K.S., Curtis, P.S., Teeri, J.A., Fogel, R., Randlett, D.L., 1993. Elevated atmospheric $\mathrm{CO}_{2}$ and feedback between carbon and nitrogen cycles. Plant and Soil 151, 105-117.

Zhang, R., Wienhold, B.J., 2002. The effect of soil moisture on mineral nitrogen, soil electrical conductivity, and $\mathrm{pH}$. Nutrient Cycling 63, 251-254.

Ziska, L.H., Emche, S.D., Johnson, E.L., George, K., Reed, D.R., Sicher, R.C., 2005. Alterations in the production and concentration of selected alkalo ids as a function of rising atmospheric carbon dioxide and air temperature: implications for ethno-pharmacology. Global Change Biology 11, 1789-1807. 


\section{| Chapter 6}

First step to integrate nematodes into food web analysis using NanoSIMS

Simone Cesarz

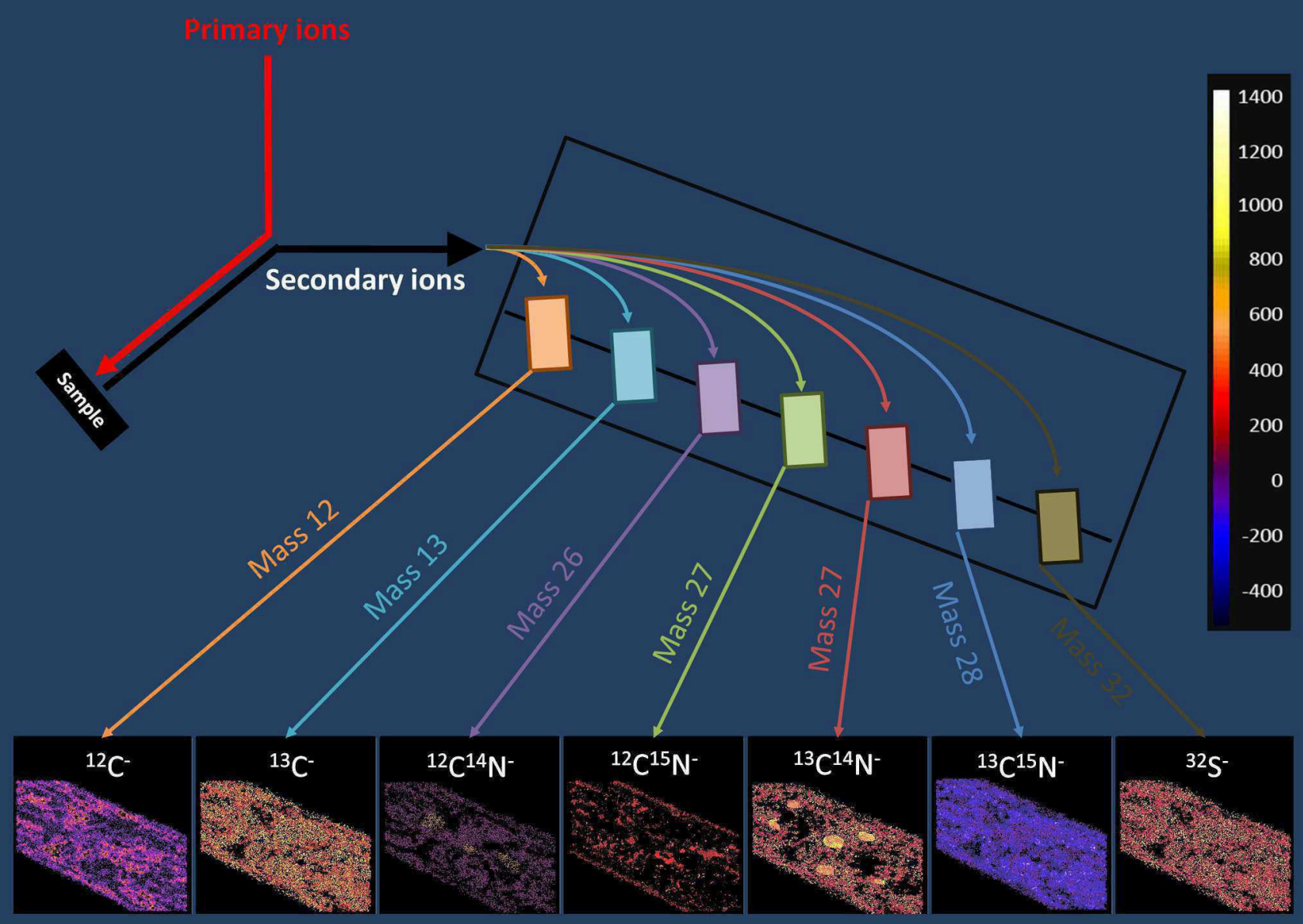




\section{Abstract}

Nematodes contribute significantly to nutrient fluxes in soil but are excluded from soil food web analyses due to their small size. NanoSIMS offe rs the possibility to trace isotopes at a high spatial resolution but its adaptability to nematodes has not been investigated so far. High vacuum during the measurement requires special embedding and was applied first according to embedding techniques for transmission electron microscopy. Single specimen of Aporcelaimellus spec. were embedded in resin and successfully measured with NanoSIMS. Simultaneous measurements of different isotopes resulted in maps of carbon, nitrogen and phosphorous. NanoSIMS did not deliver the possibili ty to integrate nematodes within food web analyses based on natural abundances due to high error in isotope detection precluding measurement of natural abundances of ${ }^{13} \mathrm{C}$ and ${ }^{15} \mathrm{~N}$ but offering a powerful tool for tracking element flow and food web relationships. Combing this approach with advanced microbiological techniques allow to differentiate species-specific bacterial and fungal sources, also tissue analyses are possible.

\section{Introduction}

Nematodes are the most abundant Metazoa worldwide (Jairajpuri \& Ahmad 1992) and contribute significantly to essential ecosystem functions such as decomposition and mineralization (Ettema 1998; Yeates et al. 2009). As nematodes are assumed to comprise mainly of bacterial, fungal and plant feeders they form an important link between basal resources and higher trophic levels. Empirical evidence for grouping nematodes into feeding groups is scarce which is unfortunate considering the enormous species numbers of between 40,000 to $10,000,000$ (Blaxter 1998). Traditionally, nematode feeding relationships are based on stoma and pharyngeal morphology arranging nemato des into feeding groups (Fig. 1), with support coming from laboratory observations (Wood 1973; Yeates et al. 1993; Okada et al. 2002, 2005; Okada \& Kadota 2003) but these observations are of limited reliability for extrapolation to field conditions. Simple stoma with fixed size are predominantly found in nematodes assumed to feed on bacteria (Fig. 1a). Permanently cavernous stoma occur mostly in predatory nematodes being expandable in size with fixed or moveable teeth to ingest large 

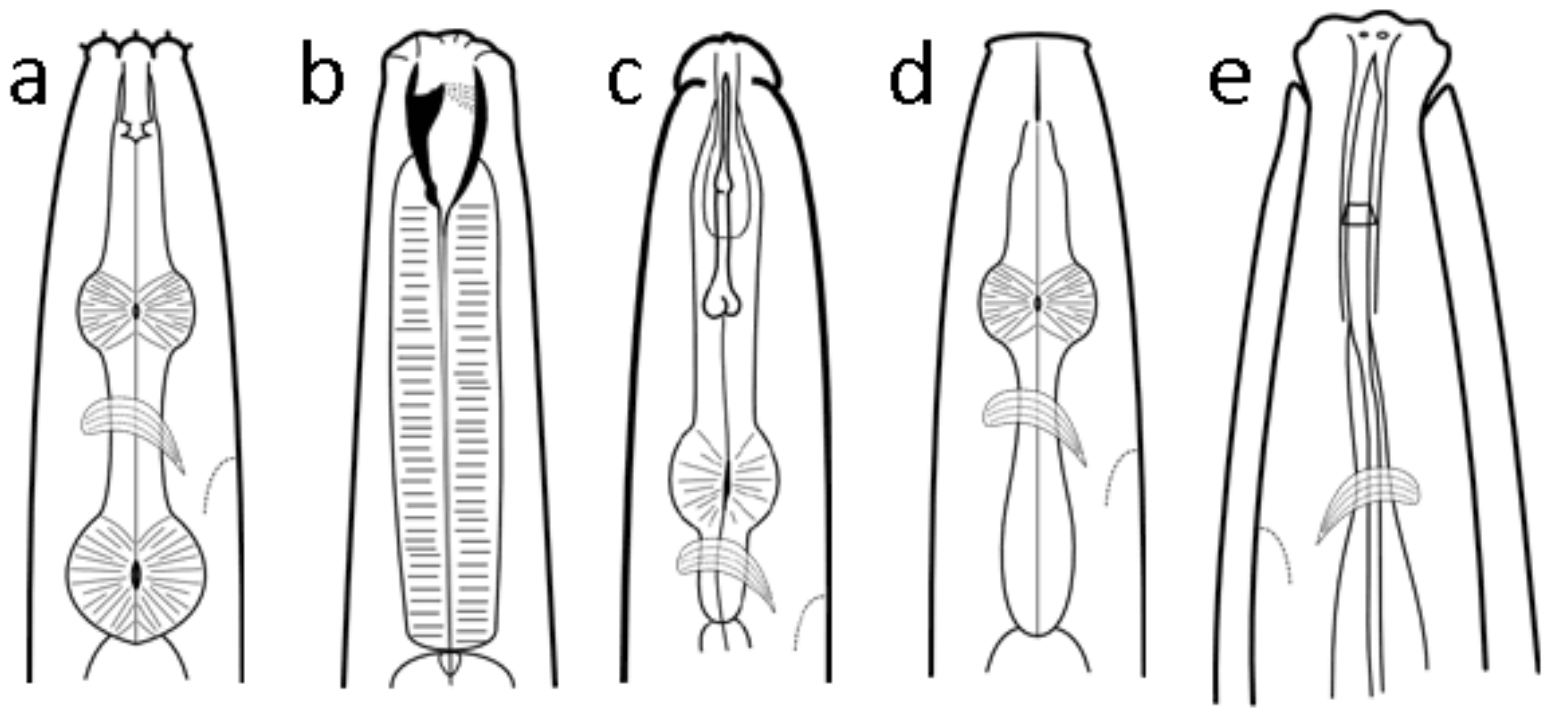

Figure 2 | Nematode feeding types of a) bacterial feeder, b) predator, c) plant feeder, d) fungal feeder and e) omnivore.

prey (Fig. 1b). Many nematodes have stylets of different sizes. The stomatostyle within the order Tylenchida presumably evolved from sclerotized parts of the stoma. It has a narrow aperture $(<0.5 \mu \mathrm{m})$ constraining the type of ingested material and is found in plant (Fig. 1c) and fungal feeders (Fig. 1d). The odontostyle of the Adenophorea originated from the wall of the esophagus and is wide open $(>5 \mu \mathrm{m})$. It is a holl ow structure and is commonly associated with predators and omnivores (Fig. 1e). Related genera within one family are assumed to be trophically similar (Bongers \& Ferris 1999), but exceptions exist (Gupta et al. 1979; Procter 1986; Small 1987; Yeates et al. 1993). Classification into ecological indices of nematodes according to their mouth morphology is popular and widespread as it is much easier as species determination. Varying sensitivity among nematodes families is used to monitor environmental disturbance and pollution, a concept realized in the Maturity Index (MI) introduced by Bongers (1990). Within the MI nematodes are classified according to their life history strategies assigning nematode families to colonizers $(c)$ or persisters $(p)$ along a scale $(c-p)$ ranging from 1 to 5 . Nematodes of $c-p 1$ are small, have a short life cycle, high fecundity, are tolerant to disturbance and can be ascribed to $r$-strategists. In contrast, nematodes of $c-p$ 5 are large, have a long life cycle combined with a long generation time and are sensitive to disturbance, therefore pursuing a $K$-strategy. Numbers between 1 and 5 reflect gradations between these opposing strategies. However, as empirical evidence for feeding relationships in soil are lacking and life history traits may vary within families, this classification systems might be of limited use Yeates et al. 2002 and need verification. 
Feeding relationships in soil are increasingly studied using stable isotope analysis (Scheu \& Falca 2000; Schmidt et al. 2004; Albers et al. 2006; Pollierer et al. 2009). However, due to their small size (300 $\mu \mathrm{m}$ to few millimeters) nematodes are excluded from terrestrial soil food web analyses as a large number of individuals is needed for one single measurement. In addition, to achieve precise information on feeding relationships determination to species level is obligatory. Sampling and determination are very time consuming and rare species cannot be included into measurements. The current limit of detection of isotopic values is in the range of 5-10 $\mathrm{g} \mathrm{N}$. For nematodes of intermediate body size of about $700 \mu \mathrm{m}$ (e.g., Mesorhabditis spec.) 340 individuals are needed for a single measurement assuming that nematodes contain about $50 \%$ carbon and $10 \%$ nitrogen of dry mass (Andrassy 1956; Buecher \& Hansen 1971; Sohlenius 1979).

To overcome size restrictions and to inspect the classification of nematodes into distinct trophic groups according to their mouth part morphology in future, NanoSIMS was tested to measure isotopic signatures within single nematode individuals. On a nano scale, observations of element flow are possible in situ. The methodology is used in microbiology (Musat et al. 2008), biomineralization (Kopp et al. 2011), cell biology (Clode et al. 2009) but also in material science and cosmochemistry. To our knowledge, it has not been employed to analyze nematode stable isotope ratios.

\section{NanoSIMS}

SIMS (ㅌecondary Ion Mass Spectrometry) is a physical method to analyze surfaces. The sample surface is bombarded with primary ions, mainly $\mathrm{Cs}^{+}$or $\mathrm{O}^{-}$depending on target secondary ions, resulting in secondary ions sputtered from the surface (Fig. 2). Due to their specific mass/charge ratio, secondary ions are differentiated in a magnetic field and are collected by a magnetic mass analyzer (Fig. 3). As nitrogen can only be detected as cyanide ion, the differentiation of ${ }^{12} \mathrm{C}^{15} \mathrm{~N}^{-}$and ${ }^{13} \mathrm{C}^{14} \mathrm{~N}^{-}$requires high mass resolution being offered by NanoSIMS $50 \mathrm{~L}$ (Cameca, Paris, France). Here, a small diameter of the primary ion beam combined with a co-axial course beam allows a resolution of $50 \mathrm{~nm}$. Consequently, stable isotopes and equal masses can be separated, but only with $\mathrm{Cs}^{+}$as primary beam. $\mathrm{Cs}^{+}$produces negatively charged ions e.g., ${ }^{12} \mathrm{C}^{-},{ }^{15} \mathrm{~N}^{13} \mathrm{C}^{-}$, whereas $\mathrm{O}^{-}$generates positively charged ions such as ${ }^{28} \mathrm{Na}^{+}$and ${ }^{40} \mathrm{Ca}^{+}$ resulting in a lower lateral resolution of $150 \mathrm{~nm}$. The possibility to detect seven masses simultaneously allows an elemental mapping within on sample and 


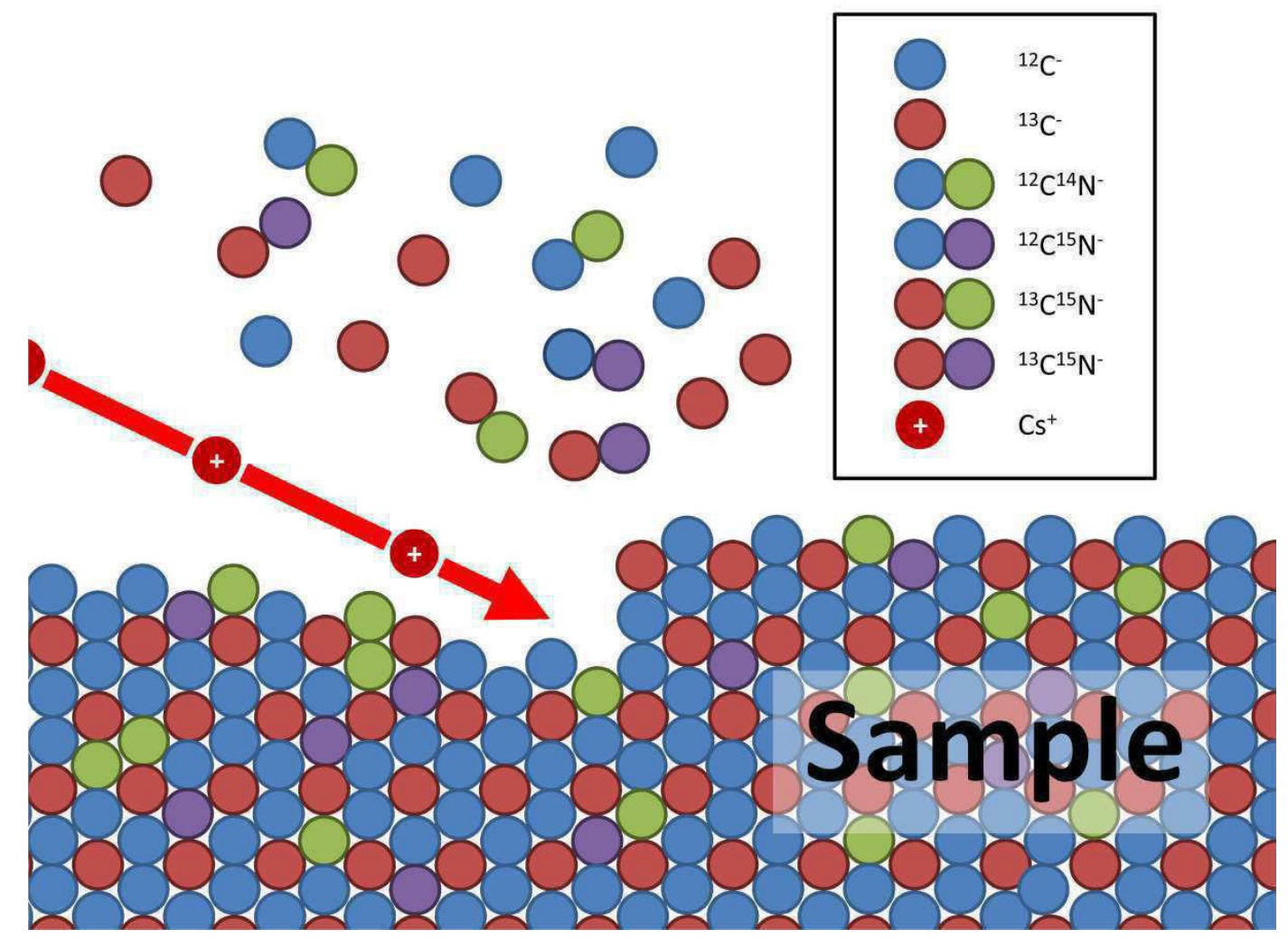

Figure 2 | Schematic illustration of a primary ion beam $\left(\mathrm{Cs}^{+}\right)$sputtering a sample resulting in negatively charged secondary ions.

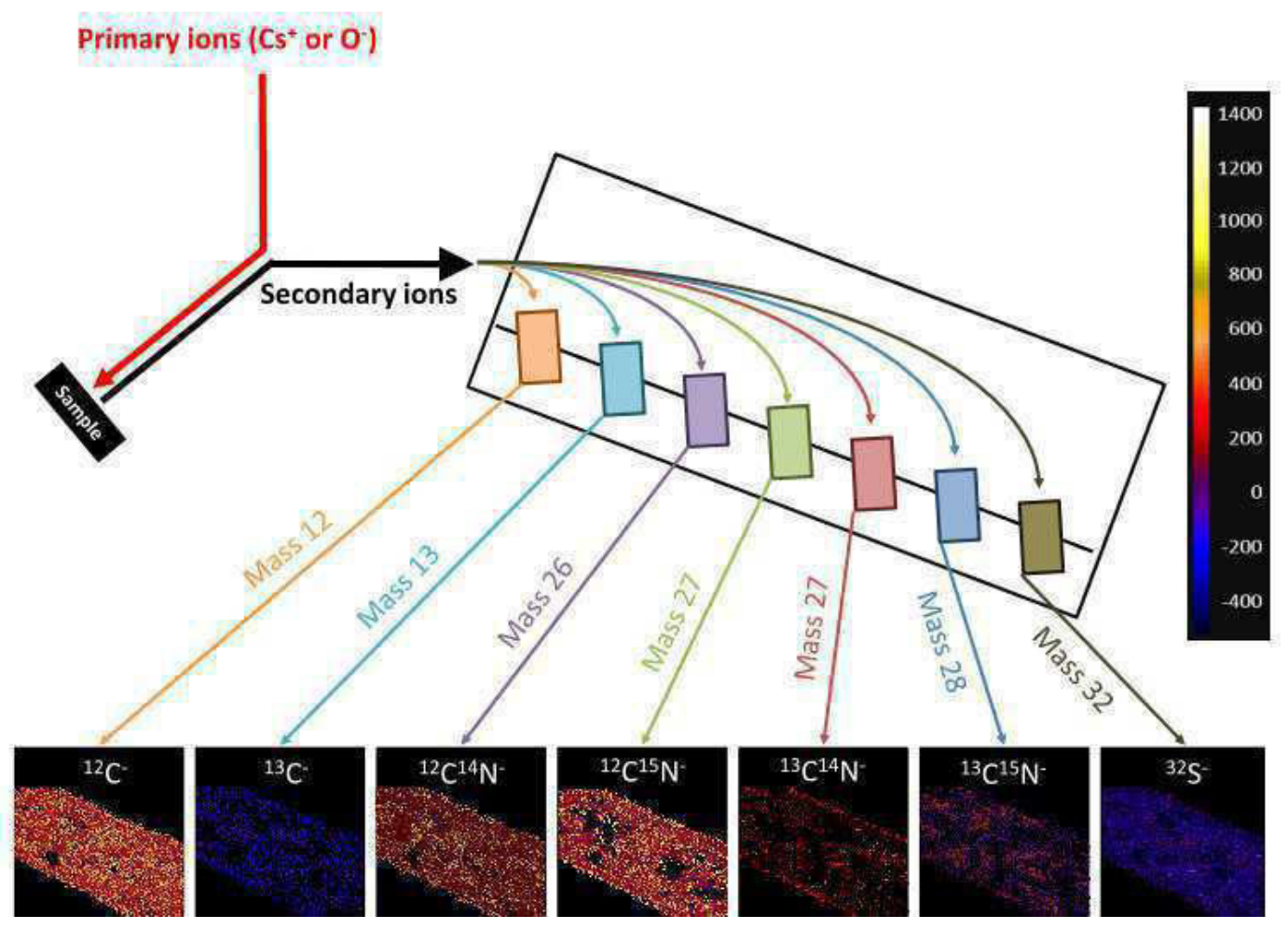

Figure 3 | Separation of seven secondary ions of different and equal masses in NanoSIMS and their frequencies displayed on a color scale. 
may help to investigate a range of different topics e.g., energy flow and metabolic rates (Musat et al. 2008). To test if it is possible to analyze nematodes with NanoSIMS single species of Aporcellaimellus spec. were prepared for measurement.

\section{Materials and methods}

\section{Nematode sampling}

Nematodes were taken from a labeling experiment from intact soil cores with beech trees fumigated with ${ }^{13} \mathrm{CO}_{2}$ and fertilized with ${ }^{15} \mathrm{NO}_{3}{ }^{15} \mathrm{NH}_{4}$ for one vegetation period. Nematodes were extracted from soil using a modified Baerman method (Ruess 1995) and stored in 4\% formaldehyde until embedding.

\section{Sample preparation}

Samples have to be attached tightly as they are subjected to high-vacuum in the NanoSIMS apparatus and had to be embedded in resin. Until now, no method is published describing an embedding technique for measuring nematodes for use in NanoSIMS. The following technique is suggested according to embedding techniques for transmission electron microscopy. It requires four different steps: fixation, dehydration, embedding and polishing.

\section{Fixation}

To preserve the structures of living organisms organic molecules have to be stabilized chemically. This step can be conducted following ne matode extraction. After nematodes were extracted from soil, nematodes were killed and fixed by formaldehyde solution (4\%) functioning as fixation agent. It has a low molecule size and penetrates single body parts of interest easily, e.g., a leg of a beetle. However, as nematode samples are intact animals and penetration may occur on only few access points, a fixation time of at least one month is suggested.

\section{Dehydration}

The use of high vacuum requires dehydration of the animals to avoid artifacts and destruction of the whole sample due to water escape. Dehydration is carried out by an increasing acetone concentration. Using a binocular under an extractor hood the supernatant of the fixation 
agent was removed carefully as the sample must not fall dry and was replaced with $40 \%$ acetone for $30 \mathrm{~min}$ until reaching propylene oxide after further ten concentration steps (Table 1). Dehydration was carried out in closed crucibles of glass to prevent the acetone from evaporate and the propylene oxide from dissolve plastic dishes.

\section{Embedding}

The supernatant of the propylene oxide was pipetted off without falling dry and was replaced according the steps listed in Table 1 using different concentrations of araldite A (Table 2). Araldite A polymerizes slowly at room temperature and can be stored in closed vessels for some days. Araldite $B$ is a mixture of araldite $A$ and a hardening agent polymerizing within few hours. Aradite B was poured in a cylindrical silico n mold with an aluminum stub at the bottom to harden at $60^{\circ} \mathrm{C}$ for $5 \mathrm{~h}$. Afterwards, the nematode was placed on it and was covered with araldite $\mathrm{B}$ for at $60^{\circ} \mathrm{C} 48 \mathrm{~h}$.

Table 1 | Steps for dehydration and embedding of nematodes in resin.

\begin{tabular}{|c|c|c|c|}
\hline Process & Concentration [\%] & Time [h] & Agent \\
\hline \multirow[t]{11}{*}{ Dehydration } & 40 & 0.5 & Acetone \\
\hline & 50 & 0.5 & Acetone \\
\hline & 70 & 0.5 & Acetone \\
\hline & 80 & 0.5 & Acetone \\
\hline & 90 & 0.5 & Acetone \\
\hline & 95 & 0.5 & Acetone \\
\hline & 100 & 0.5 & Acetone \\
\hline & 100 & 0.5 & Acetone \\
\hline & 100 & 0.5 & Acetone \\
\hline & 100 & 0.5 & Acetone \\
\hline & 100 & 0.5 & Propylene oxide \\
\hline Process & Ratio of agent used & Time $[\mathrm{h}]$ & Agent \\
\hline \multirow[t]{6}{*}{ Embedding } & $3: 1$ & 1 & Propylene oxide : Araldite A \\
\hline & $1: 1$ & overnight & Propylene oxide : Araldite A \\
\hline & $1: 3$ & 1 & Propylene oxide : Araldite A \\
\hline & $0: 1$ & overnight & Propylene oxide : Araldite A \\
\hline & $0: 1$ & 24 & Propylene oxide : Araldite A \\
\hline & $0: 1$ & 48 & Araldite A : Araldite B \\
\hline
\end{tabular}


Table 2 | Formulation of used chemicals.

\begin{tabular}{cccc}
\hline $\begin{array}{c}\text { Chemical } \\
\text { product }\end{array}$ & $\begin{array}{c}\text { Mixing } \\
\text { ratio }\end{array}$ & Chemicals & Note \\
\hline Araldite A & $1: 1$ & Araldite MCY $212:$ Hardener HY 964 & Polymerization after time \\
& & Araldite MCY 212: Hardener HY 964: & $\begin{array}{c}\text { Preparation of not less than } \\
\text { BDMA }\end{array}$ \\
\hline
\end{tabular}

\section{Polishing}

Embedded samples analyzed in NanoSIMS have to be planar and were sectioned and polished.

\section{Results}

Embedded nematodes revealed still some deficiencies in shape as their appearance was to some extent compressed (Fig. 4). Nevertheless, embedding of nematodes according to the procedure described above allowed isotopic measurements of nematodes.

Labeled nematodes had higher ${ }^{12} \mathrm{C}^{14} \mathrm{~N}$ signatures than ${ }^{12} \mathrm{C}^{15} \mathrm{~N}$ resulting in relatively low $\delta^{15} \mathrm{~N}$ signatures (Fig. 5). High counts within the tail region were due to differences in topography of the embedded nematode (Fig. 6). The opposite was true for measurements of the intestine detecting only low counts in the marked region (Fig. 7). Isotopic mapping of ${ }^{31} \mathrm{P}$ identified distal regions of the nematode without any phosphorus (Fig 7D).
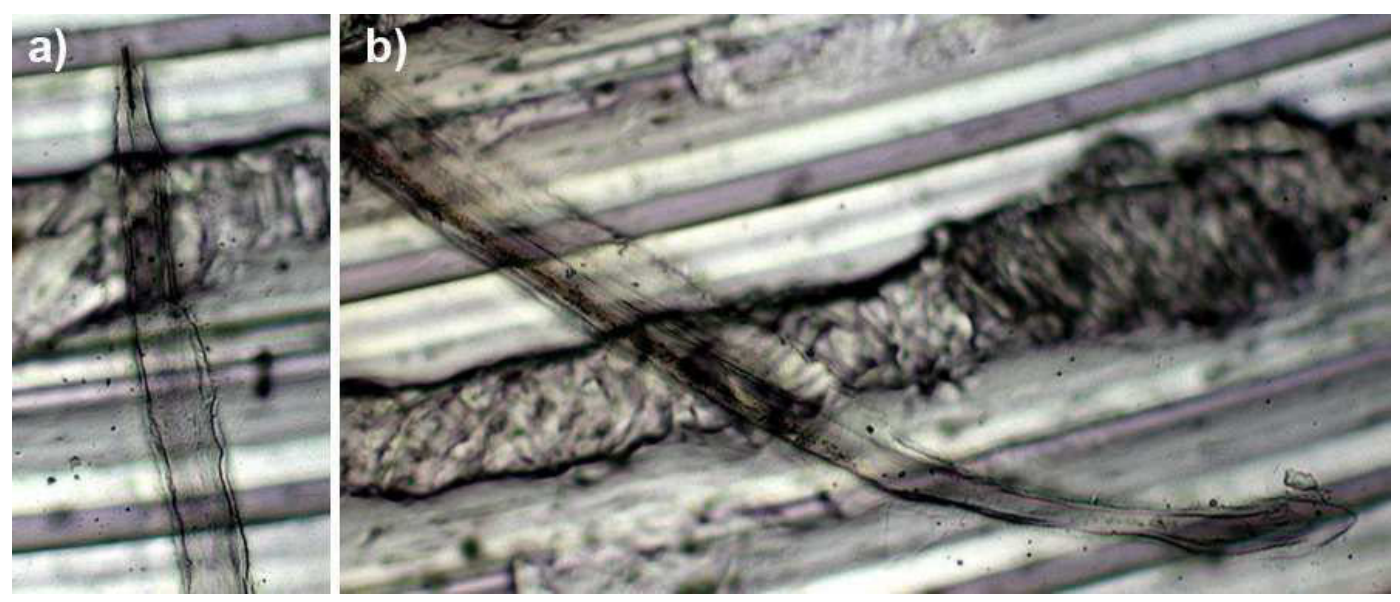

Figure 4 | Light optical microscope image of a specimen of Aporcelaimellus spec. after embedding in resin and polishing of a) the head and b) the tail. 

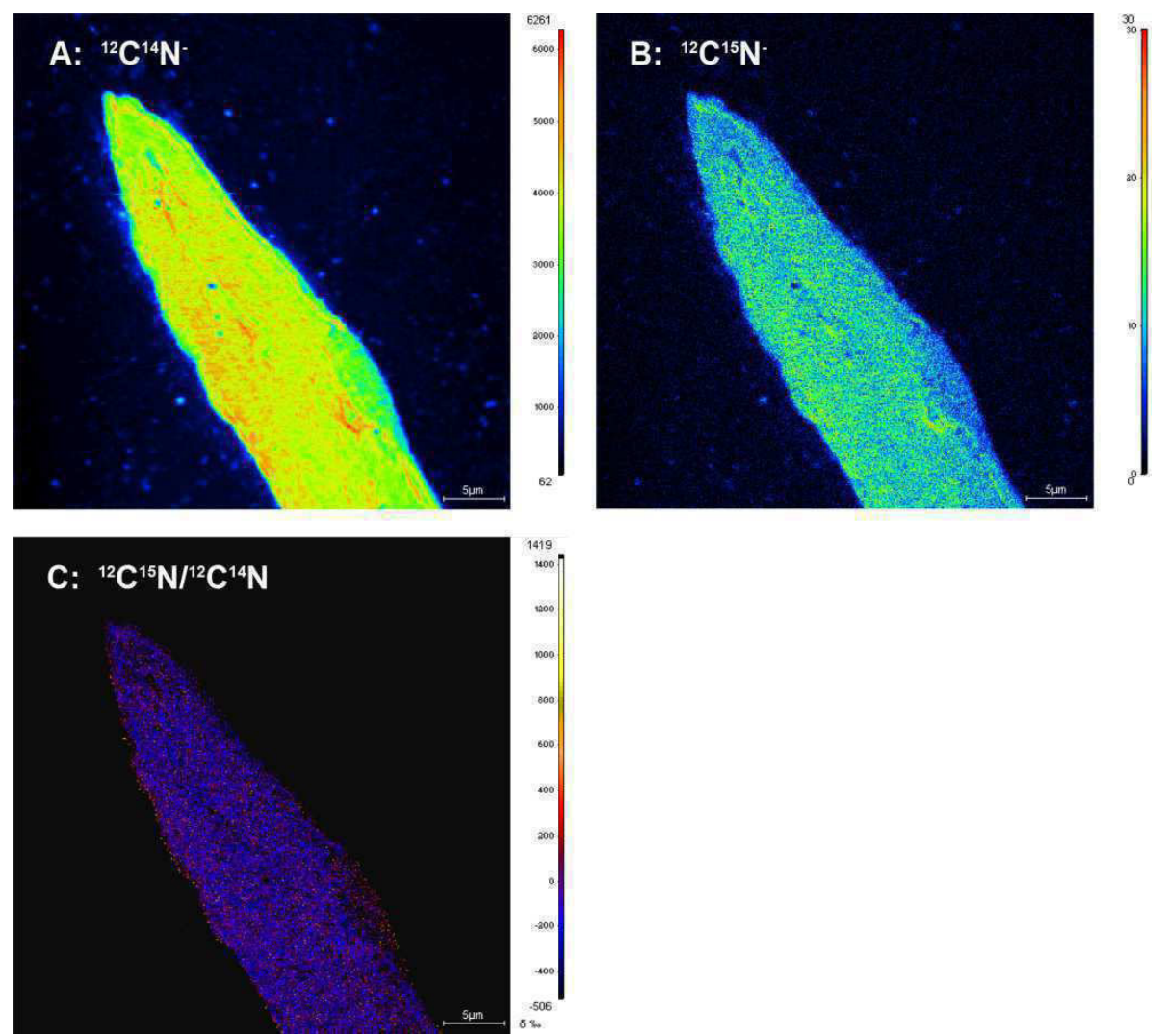

Figure 5 | The abundances of ${ }^{12} \mathrm{C}^{14} \mathrm{~N}^{-},{ }^{12} \mathrm{C}^{15} \mathrm{~N}$, and ratios of ${ }^{15} \mathrm{~N} /{ }^{14} \mathrm{~N}$ in the nematode Aporcelaimellus spec. in the head region.
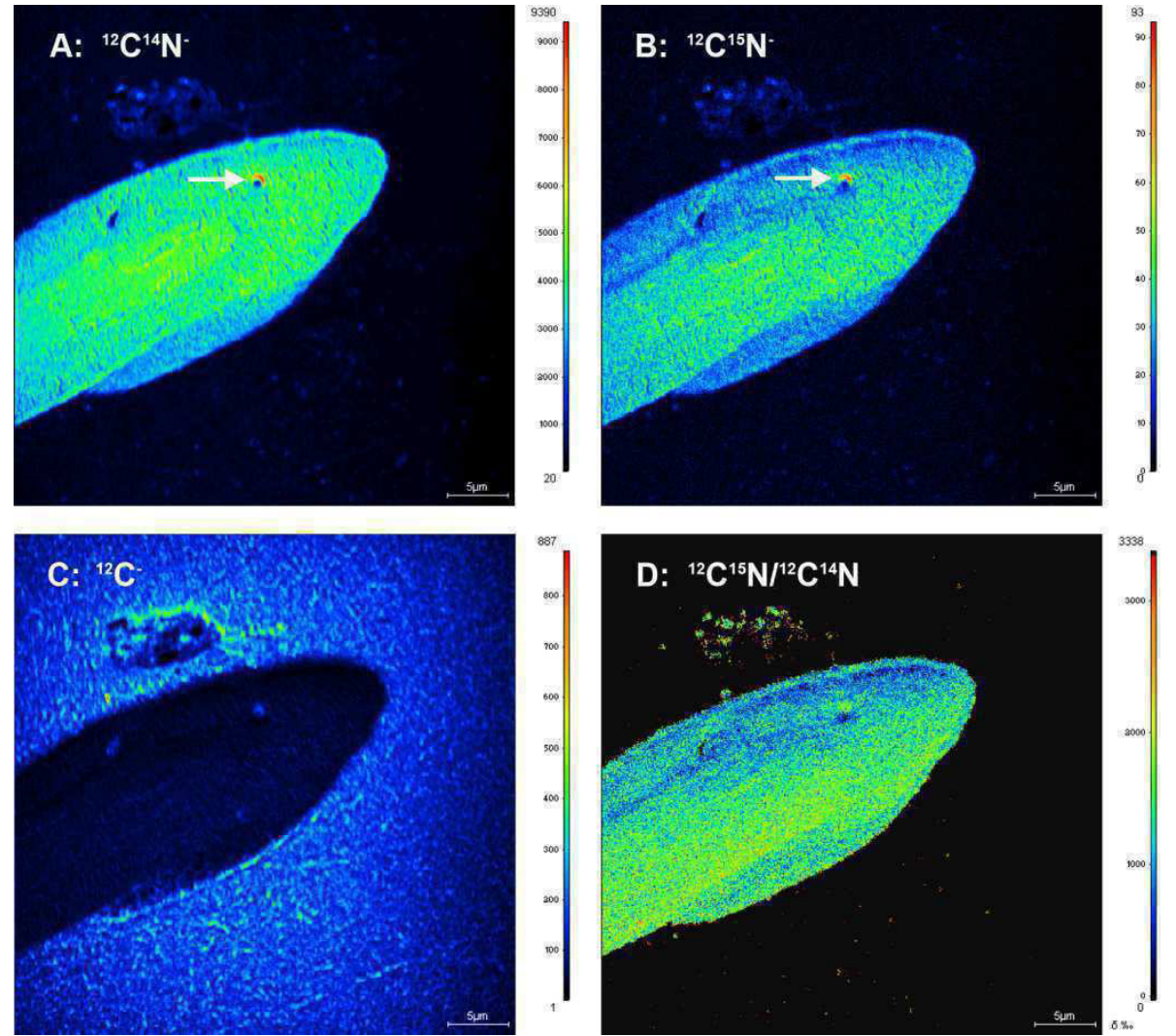

Figure 6 | The abundances of ${ }^{12} \mathrm{C}^{14} \mathrm{~N},{ }^{12} \mathrm{C}^{15} \mathrm{~N}^{-},{ }^{12} \mathrm{C}$ and ratios of ${ }^{15} \mathrm{~N} /{ }^{14} \mathrm{~N}$ in the nematode Aporcelaimellus spec. in the tail region. Arrows mark regions with high counts. 

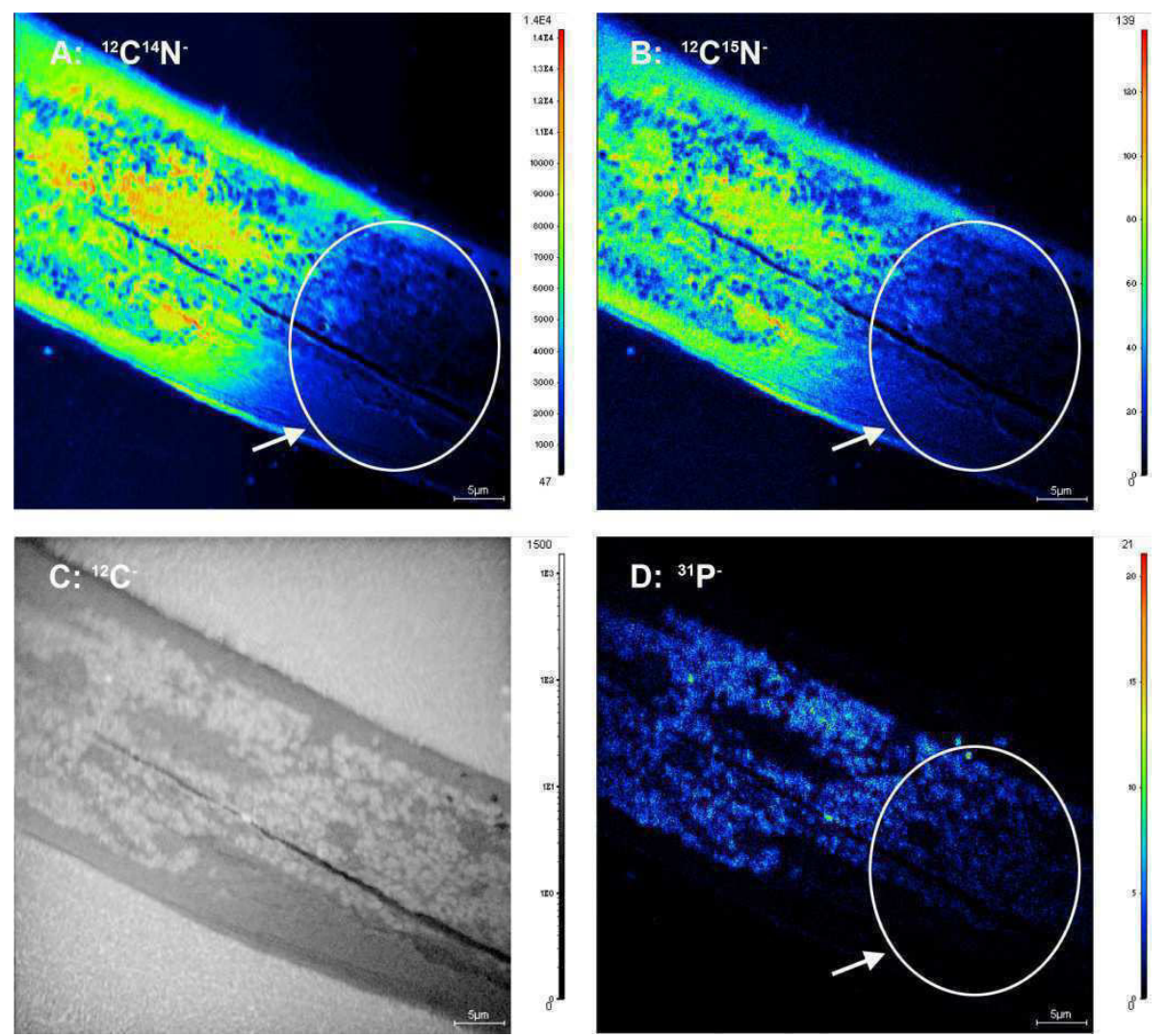

Figure 7 The abundances of ${ }^{12} \mathrm{C}^{14} \mathrm{~N}^{-},{ }^{12} \mathrm{C}^{15} \mathrm{~N}^{-},{ }^{12} \mathrm{C}^{-}$and ${ }^{31} \mathrm{P}^{-}$in the nematode Aporcelaimellus spec. in the intestine. Ellipses mark regions with low counts due to differences in topography.

\section{Discussion}

NanoSIMS allowed to measure different isotopes with in nematodes. Isotopic imaging of the nematode showed only little incorporation of ${ }^{15} \mathrm{~N}$ displayed by low $\delta^{15} \mathrm{~N}$ values. Natural abundances of $\delta^{13} \mathrm{C}$ and $\delta^{15} \mathrm{~N}$ cannot be expressed due to a high error during ion detection as only one sputtered molecule of hundreds is detected making this method not sufficient for measuring natural abundances of ${ }^{13} \mathrm{C}$. Interpretation of data using NanoSIMS requires comparisons with other samples of known isotopic value. However, a standard which represents the matrix of the nematode tissue is lacking. A possibility for validation may be bacterial cultures raised on defined ${ }^{13} \mathrm{C}$ and ${ }^{15} \mathrm{~N}$ media feed to bacterial feeding nematodes (e.g., Chaenorhabditis elegans).

High counts in the tail region (Fig. 6) and dark regions in the intestine (Fig. 7) were likely due to differences in the topography of the embedded animal as no differences were observed in $\delta^{15} \mathrm{~N}$. To overcome errors due to topography detailed images by electron microscopy are obligatory. Using the new electron microscope (Quanta 250 FEG, FEl, Eindhoven, The 
Netherlands) available at the JFB-Institute of Zool ogy and Anthropology of the University of Goettingen with an in situ ultra microtome may deliver additional information as it allows exact orientation by 3-D imaging. Lacking label of ${ }^{31} \mathrm{P}$ in distal regions can then be linked directly to specific structures within the animal.

Sample preparation was sufficient but has to be improved to achieve original body proportions. As the pre-fixation with $4 \%$ formaldehyde lasted for several months' the application time of the agents (acetone and araldite) is suggested to be increased ( 1 day per step) to penetrate the whole animal tissue completely.

\section{Fields of application}

Next to the distribution of different isotopes with in samples, NanoSIMS can be coupled with further microbiological methods. Musat et al. (2008) combined halogen in situ hybridization (HISH) with secondary ion mass spectroscopy for phy logenetic identification of bacteria and metabolic activities. Regarding nematode analysis, specific bacterial and fungal food resources may be tracked as well as possible symbiotic relationships. Nitrogen fixation by individual bacteria was imaged in the marine bivalve Lyrodus pedicellatus (Lechene et al. 2007). Here, images of single measurements were combined giving an overview of the complete animal allowing localizing the origin of $\mathrm{N}$ fixation within the bivalve. Hybridization with bacterial probes can be used to differentiate between specific bacteria as well as to differentiate bacteria labeled with different concentrations of high or light isotopes (Li et al. 2008).

To conclude, NanoSIMS can be a powerful tool to dir ectly follow energy flow in soil in situ. As physico-chemical conditions can vary strongly within only $10 \mathrm{~mm}$ of soil aggregates and root surfaces high resolution of detecting methods are urgently needed to understand processes at the nanoscale delivering spatially resolved information at biologically meaningful scales. Further, nematode indices and grouping into distinct feeding groups can be verified in future. 


\section{References}

Albers, D., Schaefer, M., Scheu, S., 2006. Incorporation of plant carbon into the soil animal food web of an arable system. Ecology 87, 235-245.

Andrassy, I., 1956. Die Rauminhalts- und Gewichtsbestimmung der Fadenwürmer Nematoda. Acta Zoologica Hungaria 2, 1-15.

Blaxter, M., 1998. Caenorhabditis elegans is a nematode. Science 282, 2041-2046.

Bongers, T., 1990. The maturity index: an ecological measure of environmental disturbance based on nematode species composition. Oecologia 83, 14-19.

Bongers, T., Ferris, H., 1999. Nematode community structure as a bioindicator in environmental monitoring. Trends in Ecology \& Evolution 14, 224-228.

Buecher, E.J., Hansen, E.L., 1971. Mass culture of axenic nematodes using continuous aeration. Journal of Nematology 3, 199-200.

Clode, P.L., Kilburn, M.R., Jones, D.L., Stockdale, E. a, Cliff, J.B., Herrmann, A.M., Murphy, D. $V$, 2009. In situ mapping of nutrient uptake in the rhizosphere using nanoscale secondary ion mass spectrometry. Plant physiology 151, 1751-7.

Ettema, C.H., 1998. Soil nematode diversity : species coexistence and ecosystem fuunction. Journal of Nematology 30, 159-169.

Gupta, M.C., Singh, R.S., Sitaramaiah, K., 1979. A new endoparasitic fungus on Xiphinema and cultivation of Rhabditis spp. and Aphelenchus a venae on same fungus. Nematologica 25, 142.

Jairajpuri, M.S., Ahmad, W., 1992. Dorylaimida, Free-living, predaceous and plant-parasitic nematodes. Oxford and IBH Publishing, New Delhi, Bombay, Calcutta.

Kopp, C., Meibom, A., Beyssay, O., Stolarski, J., Djediat, S., Szlachetko, J., Domart-Coulon, I., 2011. Calcareous sponge biomineralization: Ultrastructural and compositional heterogeneity of spicules in Leuconia johnstoni Carter, 1871. Journal of Structural Biology 173, 99-109.

Lechene, C.P., Luyten, Y., McMahon, G., Distel, D.L., 2007. Quantitative imaging of nitrogen fixation by individual bacteria within animal cells. Science 317, 1563-1566.

Li, T., Wu, T.-D., Mazéas, L., Toffin, L., Guerquin-Kern, J.-L., Leblon, G., Bouchez, T., 2008. Simultaneous analysis of microbial identity and fun ction using NanoSIMS. Environmental Microbiology 10, 580-588.

Musat, N., Halm, H., Winterholler, B., Hoppe, P., Peduzzi, S., Hillion, F., Horreard, F., Amann, R., Jørgensen, B.B., Kuypers, M.M.M., 2008. A single-cell view on the ecophysiology of 
anaerobic phototrophic bacteria. Proceedings of the National Academy of Sciences of the United States of America 105, 17861-17866.

Okada, H., Harada, H., Kadota, I., 2005. Fungal-feeding habits of six nematode isolates in the genus Filenchus. Soil Biology and Biochemistry 37, 1113-1120.

Okada, H., Kadota, I., 2003. Host status of 10 fungal isolates for two nematode species, Filenchus misellus and Aphelenchus avenae. Soil Bio logy and Biochemistry 35, 16011607.

Okada, H., Tsukiboshi, T., Kadota, I., 2002. Mycetophagy in Filenchus misellus (Andrassy, 1958) Lownsbery \& Lownsbery, 1985 (Nematoda: Tylenchidae), with notes on its morphology. Nematology 4, 795-801.

Pollierer, M.M., Langel, R., Scheu, S., Maraun, M., 2009. Compartmentalization of the soil animal food web as indicated by dual analysis of st able isotope ratios $\left({ }^{15} \mathrm{~N} /{ }^{14} \mathrm{~N}\right.$ and ${ }^{13} \mathrm{C} /{ }^{12} \mathrm{C}$ ). Soil Biology and Biochemistry 41, 1221-1226.

Procter, D., 1986. Fecundity, reproductive effort, age-specific reproductive tactics and intrinsic rate of natural increase of a high Arctic nematode belonging to the genus Chiloplacus. Holarctic Ecology 9, 104-108.

Ruess, L., 1995. Studies on the nematode fauna of an acid forest soil: spatial disturbance and extraction. Nematologica 41, 229-239.

Scheu, S., Falca, M., 2000. The soil food web of two beech forests (Fagus sylvatica) of contrasting humus type: stable isotope analysis of a macro- and a mesofaunadominated community. Oecologia 123, 285-296.

Schmidt, O., Curry, J.P., Dyckmans, J., Rota, E., Scrimgeour, C.M., 2004. Dual stable isotope analysis $\left(\delta^{13} \mathrm{C}\right.$ and $\left.\delta^{15} \mathrm{~N}\right)$ of soil invertebrates and their food sources. Pe dobiologia 48, 171-180.

Small, R.W., 1987. A review of prey of predatory soil nematodes. Pedobiologia 30, 179-206.

Sohlenius, B., 1979. A carbon budget for nematodes, rotifers and tardigrades in a Swedish coniferous forest soil. Holarctic Ecology 2, 30-40.

Wood, F.H., 1973. Nematode feeding relationships. Soil Biology and Biochemistry 5, 593601.

Yeates, G.W., Bongers, T., De Goede, R.G.M., Freckman, D.W., Georgieva, S.S., 1993. Feeding habits in soil nematode families and genera - an ou tline for soil ecologist. Journal of Nematology 25, 315-331.

Yeates, G.W., Ferris, H., Moens, T., Van der Putten, W.H., 2009. The role of nemtaodes in ecosystems, in: Wilson, M.J., Kakouli-Duarte, T. (Eds.), Nematodes as Environmental Indicators. CABI, Wallingford., pp. 1-44. 


\section{| Chapter 7}

\section{General Discussion}

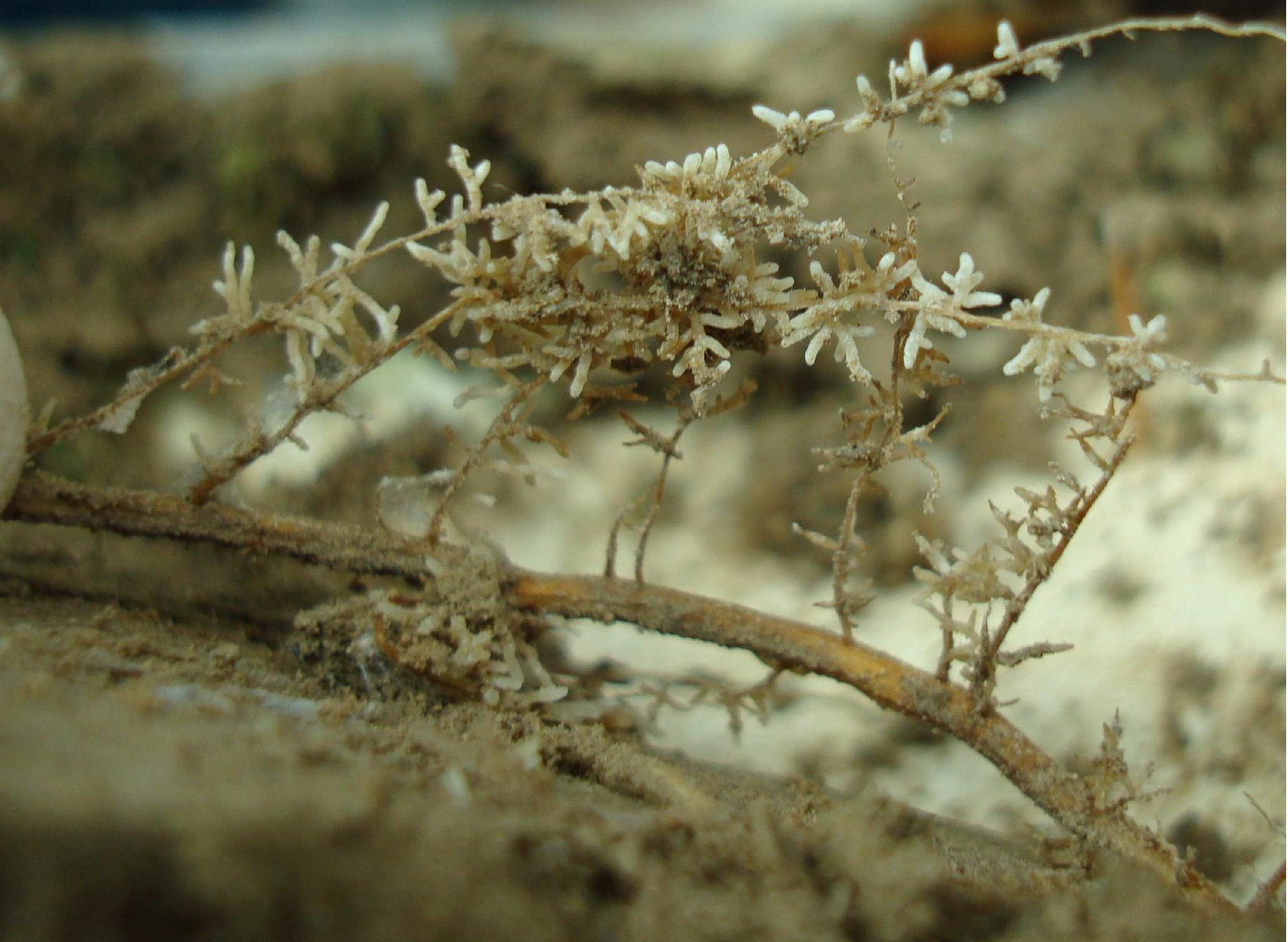




\section{Synthesis}

Gaining insight into processes taking place in soil is a precondition to understand the functioning of ecosystems. This thesis presented results on factors influencing energy flux in soil. We found that tree species identity has a species-specific effect on energy flux and the potential to affect carbon stocks in soil. Results of this thesis further highlight that global change agents interactively influence belowground communities having the potential to affect terrestrial carbon stocks. The thesis also presented first results on using NanoSIMS to follow the flux of different elements in soil.

\section{Species-specific effects on energy flux in soil}

Results of Chapter 2 and 3 highlighted that tree species identity is of major importance in structuring soil food webs and in channeling energy and surpasses effects by tree species diversity. Pure beech clusters increased fungal-feeding nematodes significantly indicating higher amounts of fungi in presence of beech. Beech is known to acidify soils (Holzwarth et al., 2011; Langenbruch et al., 2012) thereby favoring fungi (Aciego Pietri and Brookes, 2008; Rousk et al., 2009). Results of Chapter 3 indicated rhizodeposition to cause lower soil pH. Reduction of soil $\mathrm{pH}$, due to $\mathrm{H}^{+}$release, and release of organic acids by roots is a strategy to acquire nutrients (Jones et al., 2004). As beech litter is relatively recalcitrant (Jacob et al., $2009,2010)$, lowering soil pH is liekely a strategy to increase the absorbtion of nutrients from litter which are difficult to access. Positive regression between the amount of beech litter and $\mathrm{Fu}_{4}$ nematodes (Leptonchidae), being dominant in pure beech clusters, suggest that they rely on fungi colonizing litter. Lindahl et al. (2007) found saprotrophic fungi to predominate in the litter layer whereas mycorrhizal fungi to predominate in deeper layers, suggesting Leptonchidae to feed on saprothrophic rather than m ycorrhizal fungi.

In pure ash clusters fungal feeders, especially $\mathrm{Fu}_{4}$ nematodes, were strongly suppressed suggesting reduced fungal biomass and this was supported by PLFA analyses (A. Scheibe, unpublished data). High calcium and magnesium content of ash litter increased soil pH (Langenbruch et al. 2012) and this negatively influenced fungi and thereby fungal-feeding nematodes. In contrast to fungal feeders, bacterial feeding nematodes flourished in pure ash clusters. Generally, bacteria flourish at higher soil $\mathrm{pH}$, illustrating that bacteria and fungi are 
influenced opposingly by soil characteristics, especially soil pH (Aciego Pietri and Brookes, 2008; Rousk et al., 2009). Indeed, bacterial biomass was highest in pure ash clusters compared to other tree clusters as indicated by PLFA analysis (A. Scheibe, unpublished data). Interestingly, bacterial biomass did not decrease in beech clusters, however, the ratio of fungi to bacteria increased. This is in line with findings of Chapter 3 reporting no change in the bacterial biomass but an increase in the overall specific respiration. Low soil $\mathrm{pH}$ therefore is suggested not to repress bacteria but to decrease their carbon use efficiency. Bacteria have to invest more energy at low soil pH to work against high $\mathrm{H}^{+}$gradients (Rousk et al. 2009). Reduced carbon use efficiency was shown in Chapter 3 to reduce the amount of litter-derived carbon to enter higher trophic levels. In addition, the fungal marker 18:2 $\omega 6,9$ was depleted in ${ }^{13} \mathrm{C}$ as indicated by ${ }^{13} \mathrm{C}$-PLFA analysis. Low $\delta^{13} \mathrm{C}$ values indicate decomposition of structural SOM compounds as recalcitrant and more complex compounds like lignin are stronger depleted than labile compounds such as hotwater-soluble carbon (Bowling et al., 2008; Pollierer et al., 2009). Reduced carbon use efficiency in bacteria and increased SOM decomposition by fungi increased carbon loss from soil. Factors influencing carbon dynamics in forest soils are of high interest as $60 \%$ of the terrestrial carbon is stored in forests (McKinley et al., 2011).

On a global scale forest soils store twice as much carbon as the vegetation and the atmosphere together (Batjes, 1996). Changes in vegetation were regarded less important than changes in soil carbon stocks (Medlyn et al., 2005). However, results of this thesis indicate vegetation to strongly influence carbon stocks with the effects potentially reinforcing today's rising atmospheric $\mathrm{CO}_{2}$ concentrations. The influence mediated by vegetation is likely restricted to certain key species. Results from Chapter 2 reported lime to be of minor importance in influencing energy flow in soil. Also other tree species studied in the close to natural beech forest in the Hainich National Park (i.e., Carpinus betulus, Acer pseudoplatanus) revealed no effects on litter decomposition, water use, ectomycorrhizal colonization and soil properties, whereas beech and ash often had significant influence (Jacob et al., 2010; Krämer and Hölscher, 2010; Lang et al., 2011; Langenbruch et al., 2012). Also, other factors including forest management type and land use were reported to be of minor importance for structuring soil food webs but showed that strong differences between different tree species stands occur (Erdmann et al., 2012). In sum, the structure of soil food webs, and therefore the way energy is channeled through soil communities varies markedly with tree species. The basal resources 
provided by key species i.e., litter quality and root resources, control major decomposition pathways and can affect soil carbon stocks.

\section{Global change and belowground communities}

Next to tree species, increased $\mathrm{CO}_{2}$ concentrations and other global change agents are reported to affect carbon stocks in soil (Hyvönen et al., 2007). To realistically estimate alterations in carbon stocks induced by global change agents it is crucial to understand the response of the belowground system as it influences a variety of important soil processes, e.g., decomposition of organic matter and nutrient mineralization, and this may feedback to atmospheric $\mathrm{CO}_{2}$ concentrations. Changes in carbon dynamics in soil have the potential to increase atmospheric $\mathrm{CO}_{2}$ due to increased biological activity (carbon loss) or to strengthen the passive pool through reduced biological activity, physical and chemical protection (carbon sequestration) (Schulze and Freibauer, 2005). The lack of knowledge on soil organic carbon dynamics led to unrealistic estimations of carbon losses. (Bellamy et al., 2005) observed an unexpected annual carbon loss in soils across Engla nd of 13 million tons equivalent to the UK's annual $\mathrm{CO}_{2}$ reduction effort. Reasons for this marked carbon loss remain unclear, however, land use change and global change presumably are the main drivers (Schulze \& Freibauer 2005). To inspect the response of the decomposer system to carbon dynamics we took advantage of the BioCON project, where atmospheric $\mathrm{CO}_{2}$ concentrations, nitrogen deposition and precipitation were manipulated. The BioCON experiment operates since more than 13 years and investigates the effect of $\mathrm{C}$ and $\mathrm{N}$ simultaneously; further, recently also precipitation was manipulated (Reich et al., 2001; Adair et al., 2011).

Changes within the belowground community were positive, negative and neutral indicating complex processes in soil with the soil fauna being affected at different levels. Elevated atmospheric $\mathrm{CO}_{2}$ concentrations increased the energy input into soil by increasing the biomass of plants, rhizodeposition and microorganisms (Adair et al., 2009; Reich, 2009; Lee et al., 2011). However, only few taxa were affected by these effects of elevated $\mathrm{CO}_{2}$ arguing for modest alterations of the soil food web subjected to long-term exposure to elevated $\mathrm{CO}_{2}$. Interestingly, neither bacterial- nor fungal-feeding nematodes profited from increased microbial biomass at elevated $\mathrm{CO}_{2}$. The increase in plant growth at elevated $\mathrm{CO}_{2}$ can lead to 
nutrient limitations in soil and reduces the quality of the microbial biomass (Hu et al., 2001). This may explain the limited response of microbivore nematodes and suggests that food quality i.e., nutrient concentrations of microorganisms, are more important than food quantity (Schmidt et al., 2000). At high nitrogen levels, the densities of bacterial- and fungalfeeding nematodes increased significantly suggesting an increase in quality of microorganisms and bottom-up limitation of microbial feeding fauna. Other taxa were, however, negatively affected by $\mathrm{N}$ addition reducing microarthropod diversity, nematode richness, densities of predatory nematodes and herbivores. Decreases in diversity imply declines in the structure of soil food webs. This is supported by low SI at high nitrogen levels indicating reduced connectivity and only few trophic links (Ferris et al., 2001). Nitrogen addition was also shown to decrease the labile C pool delivered by plants (Dijkstra et al., 2005; Adair et al., 2009, 2011; Högberg et al., 2010) and this likely accounts for the negative effects on soil fauna. Interactions of nitrogen availability and plant derived root exudates may function as an important driver of belowground communities (Högberg et al. 2010) and therefore of the maintenance of ecosystem functions (Hooper et al., 2005; Balvanera et al., 2006).

Effects of elevated $\mathrm{N}$ were stronger than of elevated $\mathrm{CO}_{2}$. But interactions of $\mathrm{CO}_{2}$ and $\mathrm{N}$ indicated that both are interconnected and resulted in different responses of the soil fauna. Negative effects of $\mathrm{N}$ on microarthropod taxa richness were ameliorated by elevated $\mathrm{CO}_{2}$, while nematode taxa richness was at minimum at elevated $\mathrm{N}$ and $\mathrm{CO}_{2}$. Further, Astigmata, Prostigmata and fungal feeding nematodes had higher densities at elevated $\mathrm{N}$ at ambient levels of $\mathrm{CO}_{2}$, but the opposite was true at elevated nitrogen and $\mathrm{CO}_{2}$. Classification of fungal feeders into $\mathrm{Fu}_{2}$ and $\mathrm{Fu}_{4}$ nematodes revealed two contrasting responses to elevated $\mathrm{CO}_{2}$ and $\mathrm{N}$. $\mathrm{Fu}_{2}$ nematodes increased at high $\mathrm{N}$ levels, especially at ambient $\mathrm{CO}_{2}$ concentrations. In contrast, $\mathrm{Fu}_{4}$ nematodes increased at elevated $\mathrm{CO}_{2}$ concentrations, but decreased at high $\mathrm{N}$ levels and mirrored the pattern observed for AM fungi (Jifon et al., 2002; Treseder, 2004; Antoninka et al., 2011). We suggest $\mathrm{Fu}_{4}$ nematodes to predominantly feed on AM fungi and $\mathrm{Fu}_{2}$ nematodes to rely rather on saprotrophic fungi. At nutrient rich conditions, the importance of mycorrhizal fungi decreases (Antoninka et al. 2011) whereas it increases in nutrient-poor soils and at elevated $\mathrm{CO}_{2}$ conditions aggravating nutrient limitation (Franss on, 2012). Saprotrophic fungi are able to mobilize carb on from soil (Hobbie and Horton, 2007) and their increase at elevated $\mathrm{N}$ may increase SOM decomposition and therefore carbon loss from soil. However, the resistant C pool in soil increas es at elevated N (Reid et al., 2012) suggesting 
increased input of recalcitrant compounds to soil originating from increased input of plant litter at elevated $\mathrm{N}$ (Reich et al., 2001). Increased Cl at elevated $\mathrm{N}$ supports this finding and suggests that elevated $\mathrm{N}$ can foster the carbon sink in nutrient-poor soils (Reid et al. 2012).

\section{Isotope techniques}

Isotope techniques are a powerful tool to follow carbon and nitrogen through the soil food web. Advantages are provided in ${ }^{13} \mathrm{C}$-PLFA analysis allowing to follow bacterial and fungal processed carbon from basal resources to higher trophic levels (Pollierer et al., 2012). Interactions of carbon and nitrogen were shown to influence many processes, but compoundspecific PLFA techniques are restricted to follow carbon. Further advantages in tracing carbon, nitrogen and other elements are provided by the use of NanoSIMS (Chapter 6). Starting to use NanoSIMS, I obtained isotopic signatures of a single nematode for the first time. This opens a variety of new opportunities to investigate soil food webs. By measuring single individuals the variance in stable isotope signatures can be measured allowing to identify the width of trophic niches of populations. Further, the method allows to advance our knowledge in fractionation processes in animal tissue. Trophic fractionation is not equal in different taxa and trophic groups but varies with a range of factors (Tiunov, 2007; Semenina and Tiunov, 2011). Characteristics of animal and microbial cell structures can be identified when comparing elemental maps and microscopic pictures advancing our knowledge on physiological processes (Lechene et al., 2007). The combination of NanoSIMS with hybridization techniques, e.g., FISH, may allow to identify bacterial and fungal species as well as their metabolic rates (Musat et al., 2012). Relationships between microorganisms, i.e. bacterial and fungal species, and their basal resources, i.e. litter and root derived compounds, can be visualized (Li et al., 2008). Consequently, isotopic signatures and fluore scent probe labels of microorganisms can be traced into consumer tissue allowing to identify feeding relationships at species level. Changes in energy flux induced by global change agents may also be investigated by the use of NanoSIMS for identifying changes in energy flow. 


\section{Conclusion and outlook}

Although both investigated systems, i.e. forest and grassland, differ strongly in structure, plant and animal community, they provided insights into important factors influencing carbon dynamics. The idea of key species in the investigated forest is also applicable in grasslands. In the BioCON experiment four out of sixteen plant species (Achillea millefoilum, Poa pratensis, Lupinus perennis and Bromus inermis) increased in biomass suggesting to have a strong impact on carbon flow in the study system (Reich et al., 2001). Further, the nitrogen-fixing legume Trifolium pratense influenced soil $\mathrm{N}$ availability, overall plant biom ass production and carbon and nitrogen storage in grassland (De Deyn et al., 2008). In a global change context, management practices may foster sequestration of carbon in grassland thereby helping to reduce atmospheric $\mathrm{CO}_{2}$ concentrations.

The observed nutrient limitation caused by elevated $\mathrm{CO}_{2}$ in the investigated grassland also occurs in forests. Forests comprise long-lived plan ts sequestering large amounts of carbon and nutrients in their biomass and, with the input of I itter, also increasing carbon and nutrient sequestration in soil. In the long-term, this causes a progressively decreasing mineral $\mathrm{N}$ pool for plant uptake at elevated $\mathrm{CO}_{2}$ as biomass increases (Luo et al., 2004). This highlights the importance of interactions between $\mathrm{CO}_{2}$ and $\mathrm{N}$ in terrestrial ecosystems (Reich et al., 2006). The symbiosis of mycorrhizal fungi and plants to overcome nutrient restrictions is hypothesized to increase with increasing $\mathrm{CO}_{2}$ concentration in the atmosphere (Fransson and Johansson, 2010; Fransson, 2012). Diversity of species but also within species is important to allow adaptation of communities and species to chan ging environments (Hughes et al., 2008; Johnson et al., 2012). Expected changes in plant functional traits are suggested to promote carbon sequestration (De Deyn et al. 2008).

Nematodes were shown to be a useful tool to study energy flow in soil. Their diverse feeding habits allow tracing bacterial and fungal dominated processes, thereby allowing to gain insight into the structure of soil food webs. Using NanoSIMS may help to verify suggested feeding relationships of nematodes and make this tool more powerful. The combination of nematodes with NanoSIMS will foster our understanding of the structure and functioning of soil food webs which is of paramount importance in face of global change. 


\section{References}

Aciego Pietri, J.C., Brookes, P.C., 2008. Relationships between soil pH and microbial properties in a UK arable soil. Soil Biology and Biochemistry 40, 1856-1861.

Adair, E.C., Reich, P.B., Hobbie, S.E., Knops, J.M.H., 2009. Interactive effects of time, $\mathrm{CO}_{2}, \mathrm{~N}$, and diversity on total belowground carbon allocation and ecosystem carbon storage in a grassland community. Ecosystems 12, 1037-1052.

Adair, E.C., Reich, P.B., Trost, J.J., Hobbie, S.E., 2011. Elevated $\mathrm{CO}_{2}$ stimulates grassland soil respiration by increasing carbon inputs rather than by enhancing soil moisture. Global Change Biology 17, 3546-3563.

Antoninka, A., Reich, P.B., Johnson, N.C., 2011. Seven years of carbon dioxide enrichment, nitrogen fertilization and plant diversity influence arbuscular mycorrhizal fungi in a grassland ecosystem. New Phytologist 192, 200-214.

Balvanera, P., Pfisterer, A.B., Buchmann, N., He, J.-S., Nakashizuka, T., Raffaelli, D., Schmid, B., 2006. Quantifying the evidence for biodiversity effects on ecosystem functioning and services. Ecology Letters 9, 1146-1156.

Batjes, N.H., 1996. Total carbon and nitrogen in the soils of the world. European Journal of Soil Science 47, 151-163.

Bellamy, P.H., Loveland, P.J., Bradley, R.I., Lark, R.M., Kirk, G.J.D., 2005. Carbon losses from all soils across England and Wales 1978-2003. Nature 437, 245-8.

Bowling, D.R., Pataki, D.E., Randerson, J.T., 2008. Carbon isotopes in terrestrial ecosystem pools and CO2 fluxes. New Phytologist 178, 24-40.

De Deyn, G.B., Cornelissen, J.H.C., Bardgett, R.D., 2008. Plant functional traits and soil carbon sequestration in contrasting biomes. Ecology Letters 11, 516-531.

Dijkstra, F.A., Hobbie, S.E., Reich, P.B., Knops, J.M.H., 2005. Divergent effects of elevated $\mathrm{CO} 2, \mathrm{~N}$ fertilization, and plant diversity on soil $\mathrm{C}$ and $\mathrm{N}$ dynamics in a grassland field experiment. Plant and Soil 272, 41-52.

Erdmann, G., Scheu, S., Maraun, M., 2012. Regional factors rather than forest type drive the community structure of soil living oribatid mites (Acari, Oribatida). Experimental and Applied Acarology.

Ferris, H., Bongers, T., De Goede, R.G.M., 2001. A framework for soil food web diagnostics: extension of the nematode faunal analysis concept. Applied Soil Ecology 18, 13-29.

Fransson, P., 2012. Elevated $\mathrm{CO}_{2}$ impacts ectomycorrhiza-mediated forest soil carbon flow: fungal biomass production, respiration and exudatio n. Fungal Ecology 5, 85-98. 
Fransson, P.M.A., Johansson, E.M., 2010. Elevated $\mathrm{CO}_{2}$ and nitrogen influence exudation of soluble organic compounds by ectomycorrhizal root systems. FEMS Microbiology Ecology 71, 186-96.

Hobbie, E.A., Horton, T.R., 2007. Evidence that saprotrophic fungi mobilise carbon and mycorrhizal fungi mobilise nitrogen during litter decomposition. New Phytologist 173, 447-449.

Högberg, M.N., Briones, M.J.I., Keel, S.G., Metcalfe, D.B., Campbell, C., Midwood, A.J., Thornton, B., Hurry, V., Linder, S., Näsholm, T., Högberg, P., 2010. Quantification of effects of season and nitrogen supply on tree below-ground carbon transfer to ectomycorrhizal fungi and other soil organisms in a boreal pine forest. New Phytologist 187, 485-493.

Holzwarth, F.M., M, D., Flessa, H., 2011. Effects of beech and ash on small-scale variation of soil acidity and nutrient stocks in a mixed deciduo us forest. Journal of Plant Nutrition and Soil Science 174, 799-808.

Hooper, D.U., Chapin III, F.S., Ewel, J.J., Hector, A., Inchausti, P., Lavorel, S., Lawton, J.H., Lodge, D.M., Loreau, M., Naeem, S., Schmid, B., Setälä, H., Symstad, A.J., Vandermeer, J., Wardle, D.A., 2005. Effects of biodiversity on ecosystem functioning: A consensus of current knowledge. Ecological Monographs 75, 3-35.

Hu, S., Chapin, F.S., Firestone, M.K., Field, C.B., Chiariello, N.R., 2001. Nitrogen limitation of microbial decomposition in a grassland under elevated $\mathrm{CO}_{2}$. Nature 409, 188-191.

Hughes, A.R., Inouye, B.D., Johnson, M.T.J., Underwood, N., Vellend, M., 2008. Ecological consequences of genetic diversity. Ecology Letters 11, 609-623.

Hyvönen, R., Agren, G.I., Linder, S., Persson, T., Cotrufo, M.F., Ekblad, A., Freeman, M., Grelle, A., Janssens, I.A., Jarvis, P.G., Kellomäki, S., Lindroth, A., Loustau, D., Lundmark, T., Norby, R.J., Oren, R., Pilegaard, K., Ryan, M.G., Sigurdsson, B.D., Strömgren, M., Van Oijen, M., Wallin, G., 2007. The likely impact of elevated $\left[\mathrm{CO}_{2}\right]$, nitrogen deposition, increased temperature and management on carbon sequestration in temperate and boreal forest ecosystems: a literature review. New Phytologist 173, 463-480.

Jacob, M., Viedenz, K., Polle, A., Thomas, F.M., 2010. Leaf litter decomposition in temperate deciduous forest stands with a decreasing fraction of beech (Fagus sylvatica). Oecologia 164, 1083-1094.

Jacob, M., Weland, N., Platner, C., Schaefer, M., Leuschner, C., Thomas, F.M., 2009. Nutrient release from decomposing leaf litter of temperate deciduous forest trees along a gradient of increasing tree species diversity. Soil Biology and Biochemistry 41, 21222130.

Jifon, J.L., Graham, J.H., Drouillard, D.L., Syvertsen, J.P., 2002. Growth depression of mycorrhizal Citrus seedlings grown at high phosphor us supply is mitigated by elevated $\mathrm{CO}_{2}$. New Phytologist 153, 133-142. 
Johnson, D., Martin, F., Cairney, J.W.G., Anderson, I.C., 2012. The importance of individuals: intraspecific diversity of mycorrhizal plants and fungi in ecosystems. New Phytologist 194, 614-628.

Jones, D.L., Hodge, A., Kuzyakov, Y., 2004. Plant and mycorrhizal regulation of rhizodeposition. New Phytologist 163, 459-480.

Krämer, I., Hölscher, D., 2010. Soil dynammics along a tree diversity gradient in a deciduous forest in Central Germany. Ecohydrology 3, 262-271.

Lang, C., Seven, J., Polle, A., 2011. Host preferences and differential contributions of deciduous tree species shape mycorrhizal species richness in a mixed Central European forest. Mycorrhiza 21, 297-308.

Langenbruch, C., Helfrich, M., Flessa, H., 2012. Effects of beech (Fagus sy/vatica), ash (Fraxinus excelsior) and lime (Tilia spec.) on soil chemical properties in a mixed deciduous forest. Plant and Soil 352, 389-403.

Lechene, C.P., Luyten, Y., McMahon, G., Distel, D.L., 2007. Quantitative imaging of nitrogen fixation by individual bacteria within animal cells . Science 317, 1563-1566.

Lee, T.D., Barrott, S.H., Reich, P.B., 2011. Photosynthetic responses of 13 grassland species across 11 years of free-air $\mathrm{CO}_{2}$ enrichment is modest, consistent and independent of $\mathrm{N}$ supply. Global Change Biology 17, 2893-2904.

Li, T., Wu, T.-D., Mazéas, L., Toffin, L., Guerquin-Kern, J.-L., Leblon, G., Bouchez, T., 2008. Simultaneous analysis of microbial identity and fun ction using NanoSIMS. Environmental Microbiology 10, 580-588.

Lindahl, B.D., Ihrmark, K., Boberg, J., Trumbore, S.E., Högberg, P., Stenlid, J., Finlay, R.D., 2007. Spatial separation of litter decomposition an d mycorrhizal nitrogen uptake in a boreal forest. New Phytologist 173, 611-620.

Luo, Y., Su, B., Currie, W.S., Dukes, J.S., Finzi, A., Hartwig, U., Hungate, B., Mc MURTRIE, R.E., Oren, R., Parton, W.J., Pataki, D.E., Shaw, M.R., Zak, D.R., Field, C.B., 2004. Progressive Nitrogen Limitation of Ecosystem Responses to Rising Atmospheric Carbon Dioxide. BioScience 54, 731-739.

McKinley, D.C., Ryan, M.G., Birdsey, R.A., Giardina, C.P., Harmon, M.E., Heath, L.S., Houghton, R.A., Jackson, R.B., Morrison, J.F., Murray, B.C., Pataki, D.E., Skog, K.E., 2011. A synthesis of current knowledge on forests and carbon storage in the United States. Ecological Applications 21, 1902-1924.

Medlyn, B.E., Berbigier, P., Clement, R., Grelle, A., Loustau, D., Linder, S., Wingate, L., Jarvis, P.G., Sigurdsson, B.D., McMurtrie, R.E., 2005. Carbon balance of coniferous forests growing in contrasting climates: Model-based analys is. Agricultural and Forest Meteorology 131, 97-124. 
Musat, N., Foster, R., Vagner, T., Adam, B., Kuypers, M.M.M., 2012. Detecting metabolic activities in single cells, with emphasis on NanoSI MS. FEMS microbiology reviews 36 , 486-511.

Pollierer, M.M., Dyckmans, J., Scheu, S., Haubert, D., 2012. Carbon flux through fungi and bacteria into the forest soil animal food web as in dicated by compound specific $13 \mathrm{C}$ fatty acid analysis. Functional Ecology 26, 978-990.

Pollierer, M.M., Langel, R., Scheu, S., Maraun, M., 2009. Compartmentalization of the soil animal food web as indicated by dual analysis of st able isotope ratios $\left({ }^{15} \mathrm{~N} /{ }^{14} \mathrm{~N}\right.$ and ${ }^{13} \mathrm{C} /{ }^{12} \mathrm{C}$ ). Soil Biology and Biochemistry 41, 1221-1226.

Reich, P.B., 2009. Elevated $\mathrm{CO}_{2}$ reduces losses of plant diversity caused by nitrogen deposition. Science 326, 1399-1402.

Reich, P.B., Hungate, B.A., Luo, Y., 2006. Carbon-nitrogen interactions in terrestrial ecosystems in response to rising atmospheric carbon dioxide. Annual Review of Ecology, Evolution, and Systematics 37, 611-636.

Reich, P.B., Knops, J., Tilman, D., Craine, J., Ellsworth, D., Tjoelker, M., Lee, T., Wedin, D., Naeem, S., Bahauddin, D., Hendrey, G., Jose, S., Wrage, K., Goth, J., Bengston, W., 2001. Plant diversity enhances ecosystem responses to elevated $\mathrm{CO}_{2}$ and nitrogen deposition. Nature 411, 809-812.

Reid, J.P., Adair, E.C., Hobbie, S.E., Reich, P.B., 2012. Biodiversity, nitrogen deposition and $\mathrm{CO}_{2}$ affect grassland soil carbon cycling but not storage. Ecosystems.

Rousk, J., Brookes, P.C., Bååth, E., 2009. Contrasting soil pH effects on fungal and bacterial growth suggest functional redundancy in carbon mine ralization. Applied and Environmental Microbiology 75, 1589-96.

Schmidt, I.K., Ruess, L., Bååth, E., Ekelund, F., Jonasson, S., 2000. Long-term manipulation of the microbes and microfauna of two subarctic heaths by addition of fungicide, bactericide, carbon and fertilizer. Soil Biology and Biochemistry 32, 707-720.

Schulze, E.D., Freibauer, A., 2005. Carbon unlocked from soils. Nature 437, 11-12.

Semenina, E., Tiunov, A. V, 2011. Trophic fractionation (Delta(15)N) in Collembola depends on nutritional status: A laboratory experiment and mini-review. Pedobiologia 54, 101109.

Tiunov, a. V., 2007. Stable isotopes of carbon and nitrogen in soil ecological studies. Biology Bulletin 34, 395-407.

Treseder, K.K., 2004. A meta-analysis of mycorrhizal responses to nitrogen, phosphorus, and atmospheric $\mathrm{CO}_{2}$ in field studies. New Phytologist 164, 347-355. 


\section{Acknowledgements}

I owe many thanks to Stefan Scheu who I will always keep in good memory. He was and is an excellent supervisor and provided help whenever needed. He always clamped down on our meeting and offered great ideas.

I also thank Matthias Schaefer for co-supervison despite his well-deserved retirement and being always well-disposed towards me.

I am also very grateful to Liliane Ruess for introducing me to the wonderful world of nematodes, therefore protecting me against oribatid mites, and for being part of my thesis committee.

Many thanks go to Nico Eisenhauer for overall scientific support and inspiring nonse nse talks.

The Rhizoladies Ann-Catrin Fender, Friderike Beyer, Kerttu Valtanen and Birgit Pfeiffer were excellent project partners. We had a wonderful time starting with planting thousands of tree saplings, never ending weeding, watering for hours, building huge shadow roofs, sorting tons of litter and earthworms, building lit ter nets and looking all this work to be destroyed within one winter. For your future I wish you the very best!

Ohne die technische Hilfe von Christel Fischer, Guido Humpert, Ingrid Kleinhans und Susanne Böning-Klein wäre so einiges deutlich beschwerlicher gewesen. Vielen Dank Euch und Eurem Engagement.

Thanks go also to Lars Köhler and Heinz Corner for technical support at the botanical garden.

Dieter Nünchert schulde ich so einigen Dank für das mal eben schne lle Zusammenbauen von allem Möglichen.

Many thanks belong to Michael Ackermann and René Seimel-Buchinger helping me in nematode identification and for nice company during my Berlin trips.

I like to thank Carsten Müller for introducing me and inserting my nematodes to NanoSIMS. 
I also want to thank my new flat mate Bernhard Klarner for offering me a new roof over my head and for vespertine discussions on the end of our actual manuscripts and for introducing me to gamasid mites.

Kathleen Lemanski was a comfortable room and flat mate and achieved the impossible thing to share with me everyday living and working. Evelyn Gerund, Georgia Erdmann and Olga Ferlian became also excellent friends and colleagues. Special thanks go to Olga for offering me so much help during the last time of my dissertation. I hope we all won't get lost when our ways will separate.

Special thanks go to the whole AG Scheu for creating such a nice working atmosphere. Many thanks go to my fresh three mommys Merle Werner, Anne-Lisa Löck and Magdalena Schaub as well as Juliane Pfeiffer, Dagmar Bergau and Edda Rinne for switching me from being a scientist and for wonderful friendship.

Letztendlich möchte ich mich ganz herzlich bei mein en Eltern Brigitte und Peter Cesarz bedanken, die mich immer bis zum Äußersten unterstützt haben, auch wenn der Preis dafür manchmal hoch war ;)

Ein großer Dank gebührt auch meinem Bruder Marcel Cesarz, der erst gar keine Zukunftsängste aufkommen ließ, da er mir eine kaffeekochende Position in seinem in der Zukunft aufstrebendem Großunternehmen angeboten hat.

Ganz lieben Dank auch an meine Großeltern Wanda und Karl Nawrath, die immer stolz auf mich waren. 


\section{List of Publications}

\section{Published in peer-reviewed journals:}

Cesarz, S., Ruess, L., Jacob, M., Jacob, A., Schaefer, M., Scheu, S., 2013. Tree species diversity versus tree species identity: driving forces in structuring forest food webs as indicated by soil nematodes. Soil Biology and Biochemistry, d oi: 10.1016/j.soilbio.2013.02.020.

Cesarz, S., Fender, A.-C., Beyer, F., Valtanen, K., Pfeiffer, B., Gansert, D., Hertel, D., Polle, A., Daniel, R., Leuschner, C., Scheu, S., 2013. Roots from beech (Fagus sylvatica L.) and ash (Fraxinus excelsior L.) differentially affect soil microorganisms and carbon dynamics. Soil Biology and Biochemistry 61, 23-32, doi: 10.1016/j.soilbio.2013.02.003.

Eisenhauer, N., Dobies, T., Cesarz, S., Hobbie, S.E., Meyer, R.J., Worm, K., Reich, P.B., 2013. Plant diversity effects on soil food webs are stronger than those of elevated $\mathrm{CO}_{2}$ and $\mathrm{N}$ deposition in a long-term grassland experiment. Accepted. PNAS

Eisenhauer, N., Cesarz, S., Koller, R., Worm, K. \& Reich, P. B., 2012. Global change belowground: impacts of elevated $\mathrm{CO}_{2}$, nitrogen, and summer drought on soil food webs and biodiversity. Global Change Biology 18, 435-447, doi: 10.1111/j.13652486.2011.02555.x

Cesarz, S., Fahrenholz, N., Migge-Kleian, S., Platner, C. \& Schaefer, M., 2007. Earthworm communities in relation to tree diversity in a deciduous forest. European Journal of Soil Biology 43, S61-S67.

\section{Submitted:}

Cesarz, S., Reich, P.B., Scheu S., Ruess, L., Schaefer, M., Eisenhauer, N. Changes in soil processes indicated by soil nematodes: interactive responses to elevated $\mathrm{CO}_{2}$, nitrogen deposition and reduced precipitation. 


\section{Thesis declaration}

\section{Declaration of the author's own contribution to manuscripts with multiple authors:}

Chapter 2. I analyzed and collected all nematode data, wrote the manuscript and plotted all graphs and tables. Mascha Jacob provided data on litter biomass and Andreas Jacob on fine root biomass which I both analyzed on my own. The study design was developed in the framework of the Graduiertenkolleg 1086.

Chapter 3. The rhizotron study was developed in the framework of the Cluster of Excellence "Functional Biodiversity Research" (FBR) and was a joint project of Ann-Catrin Fender (data on soil chemistry), Friderike Beyer (plant data), Kerttu Valtanen (mycorrhiza data) and Birgit Pfeiffer (pyrosequencing). I took samples and analyzed data on soil animals, PLFA and microbial respiration. I also wrote the manuscript, analyzed all data and plotted all graphs. Engineering detail drawing of the rhizotrons and ro ot distribution were delivered by Bernd Raufeisen but were changed for my own purpose.

Chapter 4. This manuscript has been published in a peer-review journal and was mainly written by Nico Eisenhauer. I delivered data on nematodes.

Chapter 5. Study design was developed in the BioCON framework. Data collection was done by Nico Eisenhauer. I identified nematodes, analyzed the data, wrote the manuscript and plotted the graphs and tables.

In every case all co-authors contributed to the final version of the manuscript. 


\section{Plagiarism declaration}

I declare that I have written this doctoral thesis independently. All persons contributing to the manuscripts have been named so. All sentences or passages quoted from other people's work have been specifically acknowledged by clear cross-referencing. I have not submitted this thesis in any form for another degree at any university or institution.

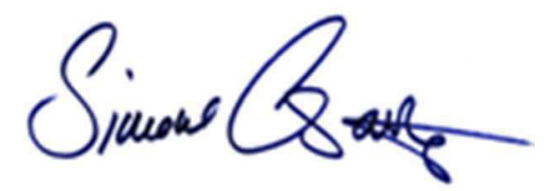

Göttingen, March 2012 